\title{
Resource assignment for adaptively modulated interconnected WLANs
}

\author{
Amiotosh Ghosh
}

A Thesis

in

The Department

of

Electrical and Computer Engineering

Presented in Partial Fulfillment of the Requirements

for the Degree of Master of Applied Science (Electrical and Computer Engineering) at Concordia University

Montreal, Quebec, Canada

June 2008

(1) Amiotosh Ghosh, 2008 


$\begin{array}{ll}\begin{array}{l}\text { Library and } \\ \text { Archives Canada }\end{array} & \begin{array}{l}\text { Bibliothèque et } \\ \text { Archives Canada }\end{array} \\ \begin{array}{l}\text { Published Heritage } \\ \text { Branch }\end{array} & \begin{array}{l}\text { Direction du } \\ \text { Patrimoine de l'édition }\end{array} \\ \begin{array}{l}\text { 395 Wellington Street } \\ \text { Ottawa ON K1A 0N4 } \\ \text { Canada }\end{array} & \begin{array}{l}\text { 395, rue Wellington } \\ \text { Ottawa ON K1A 0N4 } \\ \text { Canada }\end{array}\end{array}$

Your file Votre référence ISBN: 978-0-494-42491-9

Our file Notre référence

ISBN: 978-0-494-42491-9

NOTICE:

The author has granted a nonexclusive license allowing Library and Archives Canada to reproduce, publish, archive, preserve, conserve, communicate to the public by telecommunication or on the Internet, loan, distribute and sell theses worldwide, for commercial or noncommercial purposes, in microform, paper, electronic and/or any other formats.

The author retains copyright ownership and moral rights in this thesis. Neither the thesis nor substantial extracts from it may be printed or otherwise reproduced without the author's permission.
AVIS:

L'auteur a accordé une licence non exclusive permettant à la Bibliothèque et Archives Canada de reproduire, publier, archiver, sauvegarder, conserver, transmettre au public par télécommunication ou par l'Internet, prêter, distribuer et vendre des thèses partout dans le monde, à des fins commerciales ou autres, sur support microforme, papier, électronique et/ou autres formats.

L'auteur conserve la propriété du droit d'auteur et des droits moraux qui protège cette thèse. $\mathrm{Ni}$ la thèse ni des extraits substantiels de celle-ci ne doivent être imprimés ou autrement reproduits sans son autorisation.
In compliance with the Canadian Privacy Act some supporting forms may have been removed from this thesis.

While these forms may be included in the document page count, their removal does not represent any loss of content from the thesis.
Conformément à la loi canadienne sur la protection de la vie privée, quelques formulaires secondaires ont été enlevés de cette thèse.

Bien que ces formulaires aient inclus dans la pagination, il n'y aura aucun contenu manquant.

\section{Canada}




\begin{abstract}
$\underline{\text { ABSTRACT }}$
Resource assignment for adaptively modulated interconnected WLANs

Amiotosh Ghosh
\end{abstract}

Deployment of large wireless local area network (WLAN) in outdoor environment to provide broadband wireless access (BWA) is gaining more attention. Although several standards have been developed for this purpose numerous research and development is going on to improve the performance of the standards. Management of the wireless resource is one of the key ingredients for providing BWA to the users and total number of serviceable users in a certain area.

In this thesis we investigate WLANs performance in a metropolitan area using HiperLAN type 2 standard. Inside the LANs user can move from one place to another and user rates are dynamically adjusted based on their distance from the Access Points. To manage the wireless resources of the network we propose resource allocation schemes and evaluate their performance. We develop a generic simulation software for the network and use it for three resource allocation policies namely Minimum Overhead Round Robin (MORR), which does not depend on user's buffer condition, Weighted Minimum Overhead Round Robin (WMORR) which is a function of user buffer as well as the waiting time for transmission opportunity and Weighted Round Robin (WRR) which is a function of user buffer only. We evaluate average buffer occupancy, packet delivery time, buffer packet drop probability, overhead in downlink and uplink phases for performance comparison of the new allocation schemes. Our results show that the second adaptive resource allocation technique i.e. WMORR outperforms the other two. 


\section{Acknowledgements}

First of all I would like to express my deepest gratitude to my thesis supervisor, Dr. Ahmed K. Elhakeem, for his continuous guidance and support throughout my thesis work. During my work he always provides me key directions and inspiration with his great personality, vast experience and immense knowledge.

I want to acknowledge my fellow graduate students who helped me a lot providing continuous support and advices during my thesis work. I also want to acknowledge the faculty members, staff and system administrators of Electrical and Computer Engineering for their cooperation

I am forever indebted to my parents for their support and inspiration. 


\section{$\underline{\text { Index }}$}

List of Figures ....................................................................................................... vi

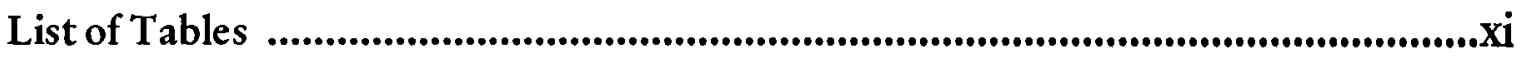

List of Abbreviations

List of symbols ................................................................................................................ xiii

Chapter 1: Introduction ............................................................................................................ 1

1.1. IEEE 802 Standards ....................................................................................................................... 1

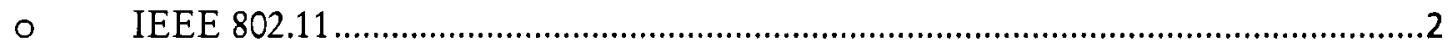

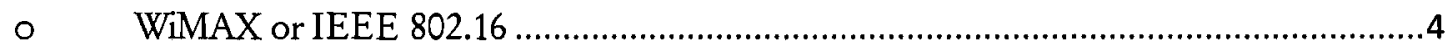

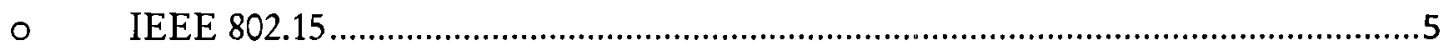

1.2. HiperLAN/2 Standard.............................................................................................................

1.3. Motivation..............................................................................................................................

1.4. Thesis objective (..................................................................................................................

1.5. Thesis organization...............................................................................................................................

Chapter 2: H/2 MAC \& Physical Layer............................................................................. 10

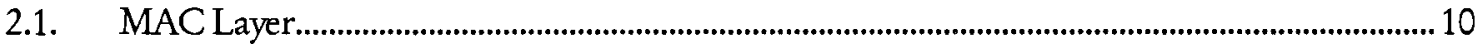

○ Broadcast phase ………………………………………......................................10

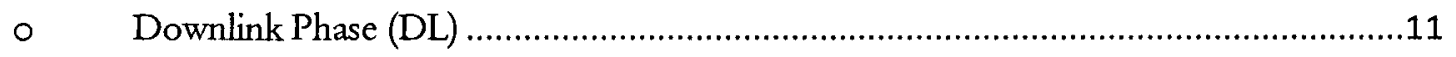

○ Uplink Phase (UL) ............................................................................................12

o Random Access phase..........................................................................................12

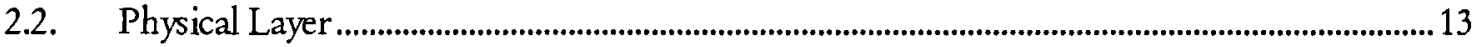

○ Frequency Band ..............................................................................................

○ Channel model...................................................................................................17

○ Radio transmission: RF carrier .............................................................................18

Chapter 3: Resource allocation policies ....................................................................20

3.1. Minimum Overhead Round Robin (MORR) ........................................................................20

3.2. Weighted Minimum Overhead Round Robin (WMORR) ...................................................20 


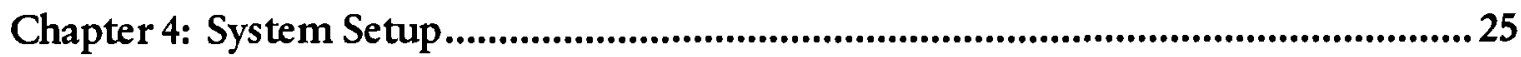

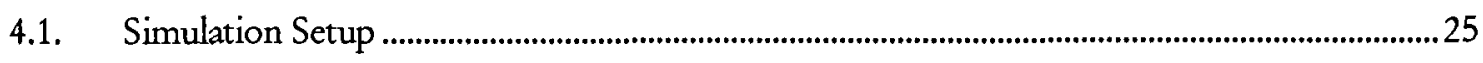

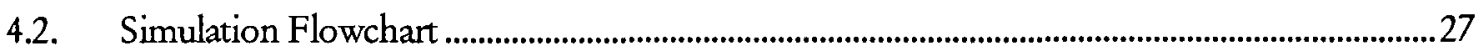

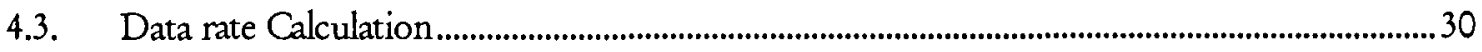

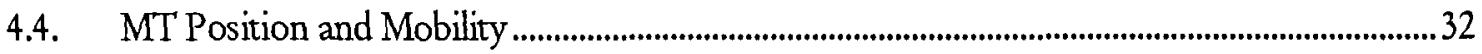

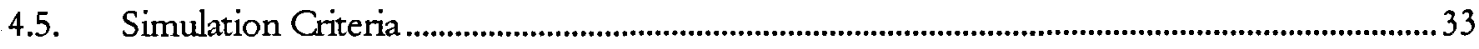

- Average Number of PDUs in Buffer and its Variance ...............................................33

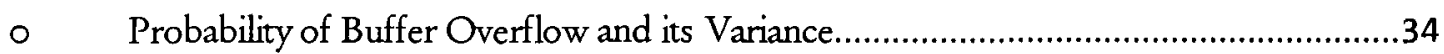

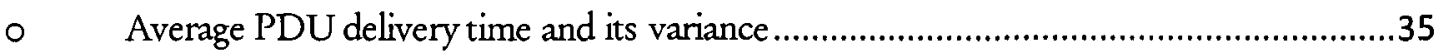

- Average Overhead in uplink phase and its variance ......................................................37

- Average Overhead in Downlink phase and its variance ...............................................37

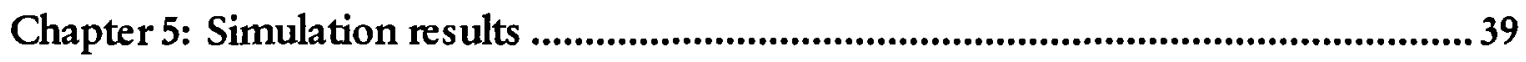

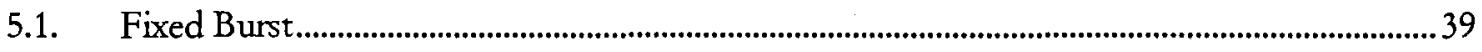

- Average Number of PDUs in Buffer and its Variance .............................................41

- Probability of Buffer Overflow and its variance ......................................................47

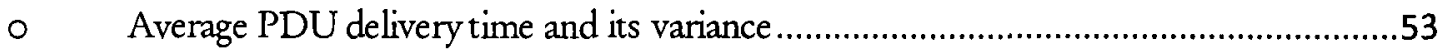

- Average overhead in Uplink phase and its variance .................................................59

- Average Overhead in Downlink Phase and its variance ..............................................65

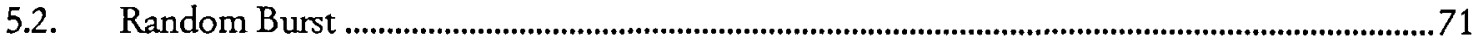

- Average Number of PDUs in Buffer and its Variance ...............................................73

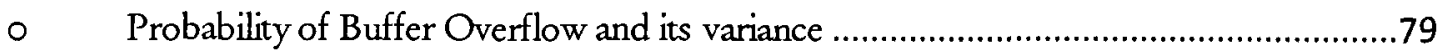

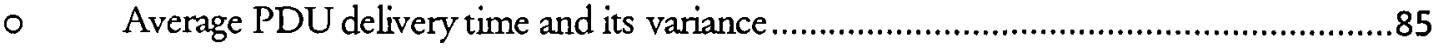

- Average Overhead in Uplink Phase and its variance ................................................91

- Average Overhead in Downlink Phase and its variance ............................................97

Chapter 6: Conclusion ...........................................................................................104

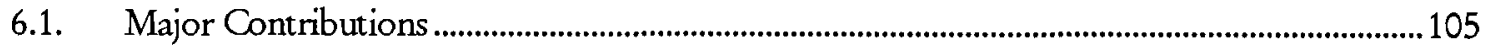

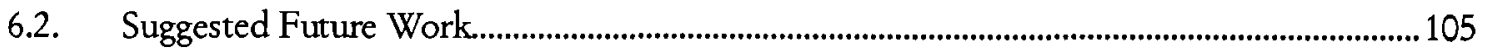

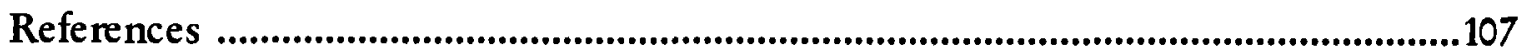




\section{$\underline{\text { List of Figures }}$}

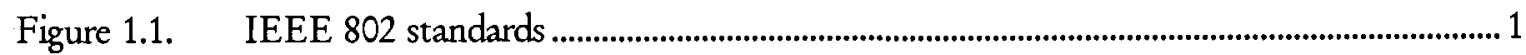

Figure 1.2. IEEE 802.11 logical architecture. .................................................................................. 3

Figure 1.3. IEEE 802.16 protocol stack ............................................................................................... 3

Figure 1.4. HiperLAN operation conditions........................................................................................ 5

Figure 1.5. HiperLAN/2 protocol stack and functions. ........................................................................

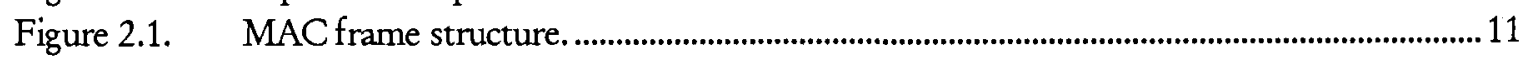

Figure 2.2. Block diagram of HiperLAN/2 physical layer....................................................................... 13

Figure 2.3. HiperLAN/2 scambler. .......................................................................................................13

Figure 2.4. H/2 physical layer BPSK, QPSK, 16QAM or 64QAM constellation points.................16

Figure 3.1. Flow chart MORR scheme. ........................................................................................................22

Figure 3.2. Flowchart for WMORR scheme .........................................................................................2

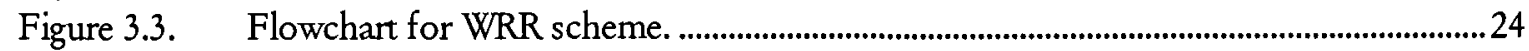

Figure 4.1. Simulation system setup. ...............................................................................................26

Figure 4.2. Flowchart for the system...............................................................................................28

Figure 4.3. Flowchart for Broadcast phase.............................................................................................28

Figure 4.4. Flowchart for Downlink phase..........................................................................................28

Figure 4.5. Flowchart for Uplink phase. ...............................................................................................29

Figure 4.6. Flowchart for $\mathrm{RCH}$ phase.............................................................................................29

Figure 4.7. PER vs $\mathrm{C} / \mathrm{N}$ curve for channel E............................................................................30

Figure 4.8. $\quad \mathrm{C} / \mathrm{N}$ Vs Distance curve for outdoor channel....................................................................32

Figure 4.9. A snapshot of transmission rate of a typical MT during simulation. ................................33

Figure 4.10. PDU delivery event under in same AP..........................................................................35

Figure 4.11. PDU delivery event under in different AP .....................................................................36

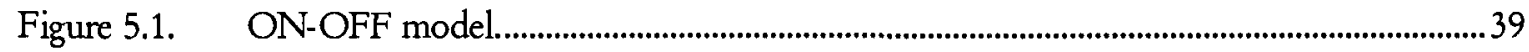

Figure 5.2. Load distribution over number of mobile terminals...........................................................40

Figure 5.3. Average number of PDUs in Buffer vs Offered load for buffer size 5 PDU at fixed

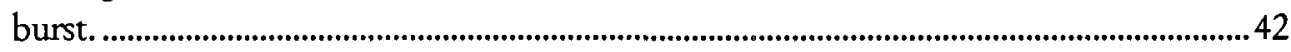

Figure 5.4. Average number of PDUs in Buffer vs Offered load for buffer size 10 PDU at fixed

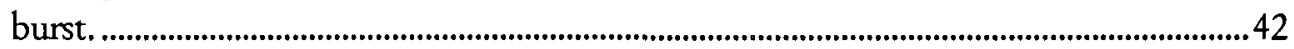

Figure 5.5. Average number of PDUs in Buffer vs Offered load for buffer size 15 PDU at fixed burst..................................................................................................................4

Figure 5.6. Average number of PDUs in Buffer vs Offered load for MORR allocation scheme at

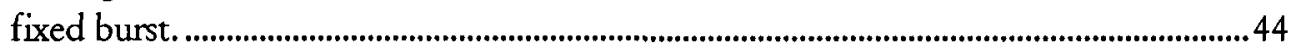

Figure 5.7. Average number of PDUs in Buffer vs Offered load for MORR allocation scheme at fixed burst. ....................................................................................................................4

Figure 5.8. Average number of PDUs in Buffer vs Offered load for MORR allocation scheme at

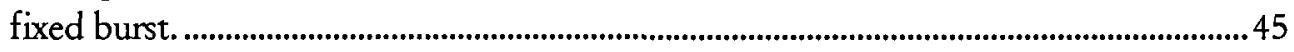

Figure 5.9. Variance in number of PDUs in Buffer vs Offered load for buffer size 5 at fixed burst. . .46 
Figure 5.10. Variance in number of PDUs in Buffer vs Offered load for buffer size 10 at fixed burst.

Figure 5.11. Variance in number of PDUs in Buffer vs Offered load for buffer size 5 at fixed burst.

Figure 5.12. Probability of buffer overflow vs offered load for buffer size 5 at fixed burst............. 48

Figure 5.13. Probability of buffer overflow vs offered load for buffer size 10 at fixed burst...........48

Figure 5.14. Probability of buffer overflow vs offered load for buffer size 5 at fixed burst............. 49

Figure 5.15. Probability of buffer overflow vs offered load for MORR allocation scheme at fixed

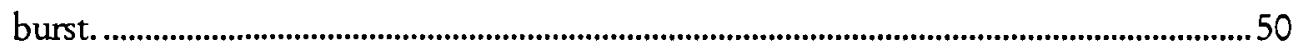

Figure 5.16. Probability of buffer overflow vs offered load for WMORR allocation scheme at fixed

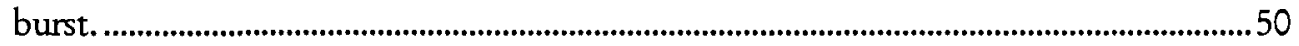

Figure 5.17. Probability of buffer overflow vs offered load for WRR allocation scheme at fixed

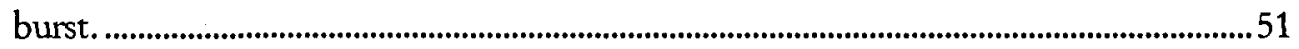

Figure 5.18. Variance in buffer overflow vs offered load for buffer size 5 at fixed burst..................52

Figure 5.19. Variance in buffer overflow vs offered load for buffer size 10 at fixed burst................52

Figure 5.20. Variance in buffer overflow vs offered load for buffer size 15 at fixed burst...............53

Figure 5.21. Average PDU delivery time vs offered load for buffer size 5 at fixed burst. .................54

Figure 5.22. Average PDU delivery time vs offered load for buffer size 10 at fixed burst...............54

Figure 5.23. Average PDU delivery time vs offered load for buffer size 15 at fixed burst...............55

Figure 5.24. Average PDU delivery time vs offered load for MORR allocation scheme at fixed

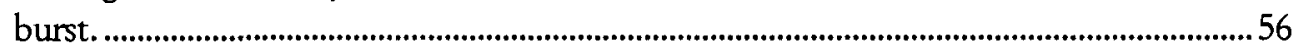

Figure 5.25. Average PDU delivery time vs offered load for WMORR allocation scheme at fixed

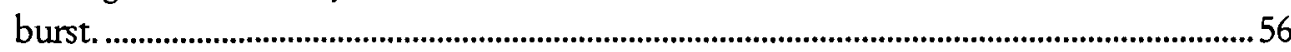

Figure 5.26. Average PDU delivery time vs offered load for WRR allocation scheme at fixed burst.

Figure 5.27. Variance in PDU delivery time vs offered load for buffer size 5 at fixed burst.............58

Figure 5.28. Variance in PDU delivery time vs offered load for buffer size 10 at fixed burst.......... 58

Figure 5.29. Variance in PDU delivery time vs offered load for buffer size 15 at fixed burst..........59

Figure 5.30. Average overhead in Uplink phase vs offered load for buffer size 5 at fixed burst. ....60

Figure 5.31. Average overhead in Uplink phase vs offered load for buffer size 10 at fixed burst...60

Figure 5.32. Average overhead in Uplink phase vs offered load for buffer size 15 at fixed burst...61

Figure 5.33. Average overhead in Uplink phase vs offered load for MORR scheme at fixed burst.

Figure 5.34. Average overhead in Uplink phase vs offered load for WMORR scheme at fixed burst.

Figure 5.35, Average overhead in Uplink phase vs offered load for WRR scheme at fixed burst...63

Figure 5.36. Variance in overhead of uplink phase vs offered load for buffer size 5 at fixed burst.64

Figure 5.37. Variance in overhead of uplink phase vs offered load for buffer size 10 at fixed burst.

Figure 5.38. Variance in overhead of uplink phase vs offered load for buffer size 15 at fixed burst.

Figure 5.39. Average overhead in downlink phase vs offered load for buffer size 5 at fixed burst. 66

Figure 5.40. Average overhead in downlink phase vs offered load for buffer size 10 at fixed burst. 
Figure 5.41. Average overhead in downlink phase vs offered load for buffer size 15 at fixed burst.

Figure 5.42. Average overhead in downlink phase vs offered load for MORR scheme at fixed burst.

Figure 5.44 . Average overhead in downlink phase vs offered load for WRR scheme at fixed burst.
Figure 5.43. Average overhead in downlink phase vs offered load for WMORR scheme at fixed burst.

Variance in overhead of downlink phase vs offered load for buffer size 5 at fixed burst. .

Figure 5.46. Variance in overhead of downlink phase vs offered load for buffer size 10 at fixed burst.

Figure 5.45.

Variance in overhead of downlink phase vs offered load for buffer size 5 at fixed burst.

Figure 5.48. Load distribution over number of mobile terminals..

Figure 5.49. Average number of PDUs in buffer vs offered load for buffer size 5 at random burst.

Figure 5.50.

Average number of PDUs in buffer vs offered load for buffer size 10 at random burst.

Figure 5.51.

Average number of PDUs in buffer vs offered load for buffer size 15 at random burst.

Figure 5.52.

Average number of PDUs in buffer vs offered load for MORR scheme at random burst.

Figure 5.53. Average number of PDUs in buffer vs offered load for WMORR at random burst... 76

Figure 5.54.

Figure 5.55 .

Figure 5.56. Average number of PDUs in buffer vs offered load for WRR at random burst...........77 Variance in number of PDUs in buffer vs offered load for buffer size 5 at random

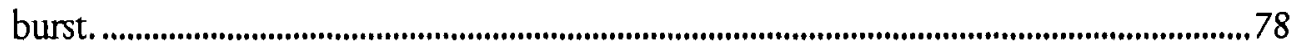

Variance in number of PDUs in buffer vs offered load for buffer size 10 at random burst. .78

Figure 5.57. Variance in number of PDUs in buffer vs offered load for buffer size 15 at random burst.

Figure 5.58. Probability of buffer overflow vs offered load for buffer size 5 at random burst........ 80

Figure 5.59. Probability of buffer overflow vs offered load for buffer size 10 at random burst...... 80

Figure 5.60. Probability of buffer overflow vs offered load for buffer size 15 at random burst...... 81

Figure 5.61. Probability of buffer overflow vs offered load for MORR scheme at random burst..82

Figure 5.62. Probability of buffer overflow vs offered load for WMORR scheme at random burst.

Figure 5.63. Probability of buffer overflow vs offered load for WRR scheme at random burst.......83

Figure 5.64. Variance in buffer overflow vs offered load for buffer size 5 at random burst............84

Figure 5.65. Variance in buffer overflow vs offered load for buffer size10 at random burst............84

Figure 5.66. Variance in buffer overflow vs offered load for buffer size 15 at random burst........... 85

Figure 5.67. Average PDU delivery time vs offered load for buffer size 5 at random burst. ............ 86

Figure 5.68. Average PDU delivery time vs offered load for buffer size 10 at random burst...........86

Figure 5.69. Average PDU delivery time vs offered load for buffer size 15 at random burst.......... 87

Figure 5.70. Average PDU delivery time vs offered load for MORR scheme at random burst....... 88 
Figure 5.71. Average PDU delivery time vs offered load for WMORR scheme at random burst...88

Figure 5.72. Average PDU delivery time vs offered load for WRR scheme at random burst..........89

Figure 5.73. Variance in PDU delivery time vs offered load for buffer size 5 at random burst....... 90

Figure 5.74. Variance in PDU delivery time vs offered load for buffer size 10 at random burst.....90

Figure 5.75. Variance in PDU delivery time vs offered load for buffer size 15 at random burst.....91

Figure 5.76. Average overhead in uplink phase vs offered load for buffer size 5 at random burst. 92

Figure 5.77. Average overhead in uplink phase vs offered load for buffer size 10 at random burst.

Figure 5.78. Average overhead in uplink phase vs offered load for buffer size 15 at random burst.

Figure 5.79. Average overhead in uplink phase vs offered load for MORR scheme at random burst.

Figure 5.80. Average overhead in uplink phase vs offered load for WMORR scheme at random

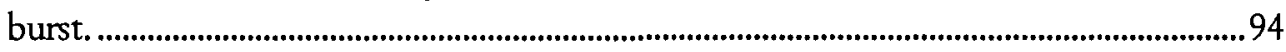

Figure 5.81. Average overhead in uplink phase vs offered load for WRR scheme at random burst.

Figure 5.82. Variance in overhead of uplink phase vs offered load for buffer size 5 at random

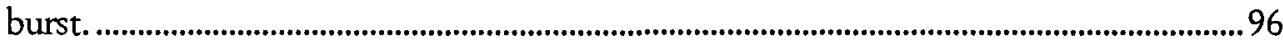

Figure 5.83. Variance in overhead of uplink phase vs offered load for buffer size 10 at random

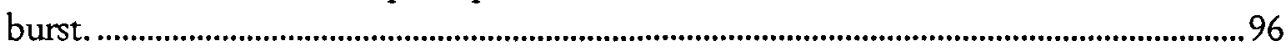

Figure 5.84. Variance in overhead of uplink phase vs offered load for buffer size 15 at random

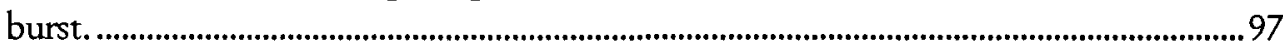

Figure 5.85. Average overhead in downlink phase vs offered load for buffer size 5 at random burst.

Figure 5.86. Average overhead in downlink phase vs offered load for buffer size 10 at random

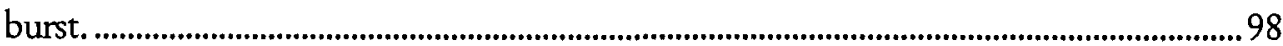

Figure 5.87. Average overhead in downlink phase vs offered load for buffer size 15 at random burst.

Figure 5.88. Average overhead in downlink phase vs offered load for MORR scheme at random

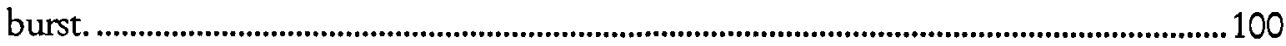

Figure 5.89. Average overhead in downlink phase vs offered load for WMORR scheme at random

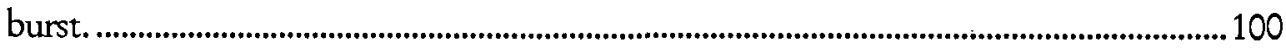

Figure 5.90. Average overhead in downlink phase vs offered load for WRR scheme at random

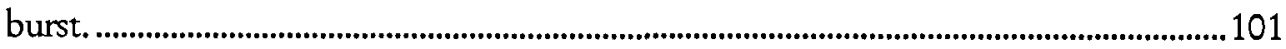

Figure 5.91. Variance in overhead of downlink phase vs offered load for buffer size 5 at random burst.

Figure 5.92. Variance in overhead of downlink phase vs offered load for buffer size 10 at random burst.

Figure 5.93. Variance in overhead of downlink phase vs offered load for buffer size 5 at random burst. 


\section{$\underline{\text { List of Tables }}$}

Table 1.1. Comparison among IEEE 802.16, 802.16a, 802.16-2004 and 802.16e. .................. 4

Table 2.1. H/2 physical layer Modes........................................................................................... 14

Table 2.2. Modulation dependent normalization factor $\mathrm{K}_{\mathrm{MOD}}$........................................................14

Table 2.3. Encoding tables for BPSK, QPSK, 16QAM and 64QAM. ..............................................15

Table 2.4. OPDM parameters................................................................................................................ 17

Table 2.5. H/2 Operating Conditions......................................................................................... 17

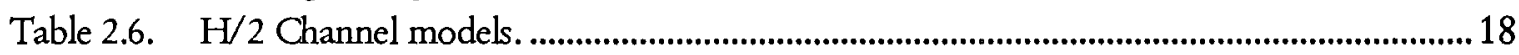

Table 2.7. Nominal carrier frequency allocation table for $\mathrm{H} / 2$ in Europe........................................ 19

Table 4.1. Operation Parameter Setting ........................................................................................2

Table 4.2. Look up table for Mode selection........................................................................................

Table 5.1. Simulation Parameters for Fixed Burst.............................................................................41

Table 5.2. Simulation Parameters for Random Burst.........................................................................72 


\section{List of Abbreviations}

\begin{tabular}{|c|c|}
\hline AP & Access Point \\
\hline $\mathrm{ACH}$ & Access feedback CHannel \\
\hline $\mathrm{ACF}$ & Association Control Function \\
\hline $\mathrm{ARQ}$ & Automatic Repeat Request \\
\hline $\mathrm{BC}$ & Broadcast Phase \\
\hline $\mathrm{BCH}$ & Broadcast Channel \\
\hline BPSK & Binary Phase Shift Keying \\
\hline BWA & Broadband Wireless Access \\
\hline $\mathrm{CC}$ & Central Controller \\
\hline CEPT & European Conference of Postal and Telecommunications Administrations \\
\hline $\mathrm{CL}$ & Convergence Layer \\
\hline $\mathrm{CRC}$ & Cyclic Redundancy Check \\
\hline CSMA/CA & Carrier Sense Multiple Access with Collision Avoidance \\
\hline DFS & Dynamic Frequency Selection \\
\hline DiL & Direct Link \\
\hline DL & Downlink \\
\hline DLC & Data Link Control \\
\hline DLOC & DLC Connection \\
\hline DLOCID & DLC Connection Identifier \\
\hline $\mathrm{DM}$ & Direct Mode \\
\hline EC & Error Control \\
\hline EIRP & Equivalent Isotropic Radiated Power \\
\hline ETSI & European Telecommunication Standardization Institute \\
\hline FCC & Federal Communications Commission (USA) \\
\hline $\mathrm{FCH}$ & Frame CHannel \\
\hline FEC & Forward Error Correction \\
\hline FFT & Fast Fourier Transform \\
\hline $\mathrm{FCH}$ & Frame $\mathrm{CH}$ Hannel \\
\hline FIFO & First In First Out \\
\hline IE & Information Element \\
\hline IEEE & Institute of Electrical and Electronic Engineers \\
\hline IFFT & Inverse Fast Fourier Transform \\
\hline IP & Internet Protocol \\
\hline $\mathrm{LCH}$ & Long transport CHannel \\
\hline LLC & Logical Link Control \\
\hline LOS & Line of Sight \\
\hline LUT & Look Up Table \\
\hline MAC & Medium Access Control \\
\hline MACID & MAC Identifier \\
\hline MORR & Minimum Overhead Round Robin \\
\hline MT & Mobile terminal \\
\hline NLOS & Non Line Of Sight \\
\hline OFDM & Orthogonal Frequency Division Multiplexing \\
\hline OSI & Open System Interconnection \\
\hline PER & PDU Error Ratio \\
\hline PDU & Protocol Data Unit \\
\hline PHY & Physical \\
\hline QAM & Quadrature Amplitude Modulation \\
\hline QoS & Quality of Service \\
\hline
\end{tabular}




$\begin{array}{ll}\text { QPSK } & \text { QuaternaryPhase Shift Keying } \\ \text { RA } & \text { Random Access } \\ \text { RCH } & \text { Random access Channel } \\ \text { RF } & \text { Radio Frequency } \\ \text { RLC } & \text { Radio Link Control Protocol } \\ \text { RR } & \text { Resource Request } \\ \text { RRC } & \text { Radio Resource Control } \\ \text { SAP } & \text { Service Access Point } \\ \text { SCH } & \text { Short transport CHannel } \\ \text { UL } & \text { Uplink } \\ \text { TDD } & \text { Time Division Duplex } \\ \text { TDMA } & \text { Time Division Multiple Access } \\ \text { WLAN } & \text { Wireless Local Area Network } \\ \text { WiMAX } & \text { Worldwide Interoperability for Microwave Access } \\ \text { WMORR } & \text { Weighted Minimum Overhead Round Robin } \\ \text { WPAN } & \text { Wireless Personal Area Network } \\ \text { WRR } & \text { Weighted Round Robin } \\ & \end{array}$

\section{$\underline{\text { List of symbols }}$}

$\begin{array}{ll}\mathrm{f}_{\mathrm{c}} & \text { Carrier Frequency } \\ \mathrm{K}_{M O D} & \text { Modulation dependent normalisation factor } \\ \mathrm{CW} & \text { Contention window } \\ \mathrm{n} & \text { Total number of RCH phase } \\ \mathrm{a} & \text { Total number of retransmission } \\ \mathrm{r}_{\mathrm{a}} & \text { RCH slot number } \\ \mathrm{f}_{\mathrm{s}} & \text { Sampling frequency } \\ \mathrm{T}_{\mathrm{u}} & \text { Useful symbol part duration } \\ \mathrm{T}_{\mathrm{cp}} & \text { Cyclic prefix duration } \\ \mathrm{T}_{\text {Total }} & \text { Total symbol duration } \\ \Delta \mathrm{f} & \text { Sub-carrier spacing } \\ \mathrm{n}_{\text {carrier }} & \text { Carrier number } \\ \mathrm{B}_{\mathrm{MTi}} & \text { Number of PDUs in the buffer of MT } \\ \mathrm{W}_{\mathrm{MTi}} & \text { Waiting time for transmission opportunity of MT } \\ \mathrm{T}_{\mathrm{Frame}} & \text { Duration of Uplink or Downlink phase of TDMA frame } \\ \mathrm{N} & \text { Noise floor } \\ \mathrm{NF} & \text { Noise factor } \\ \mathrm{S}_{\mathrm{R}} & \text { Received Signal Strength } \\ \mathrm{P}_{\mathrm{L}} & \text { Path loss } \\ \mathrm{S}_{\mathrm{T}} & \text { Transmitted Signal Strength } \\ \mathrm{d} & \text { Distance } \\ \mathrm{d} / \mathrm{N} & \text { Carrier to noise ratio } \\ \mathrm{U}(0,1) & \text { Uniform random number generator between } 0 \text { and 1 }\end{array}$




\section{Chapter 1:Introduction}

Huge demand on 'anywhere any moment connection requirement' has led to numerous research and development effects for wireless technologies. As an outcome of these efforts, wireless local area network (WLAN) standards have offered the capability of delivering all the services possible in wired infrastructure. Wireless technologies are replacing and complementing their wired counterpart due to their flexibility, equivalent performance and user friendly devices. This led to advancement and improvement of a number of standards developed by different standardization body such as IEEE, ETSI, etc.

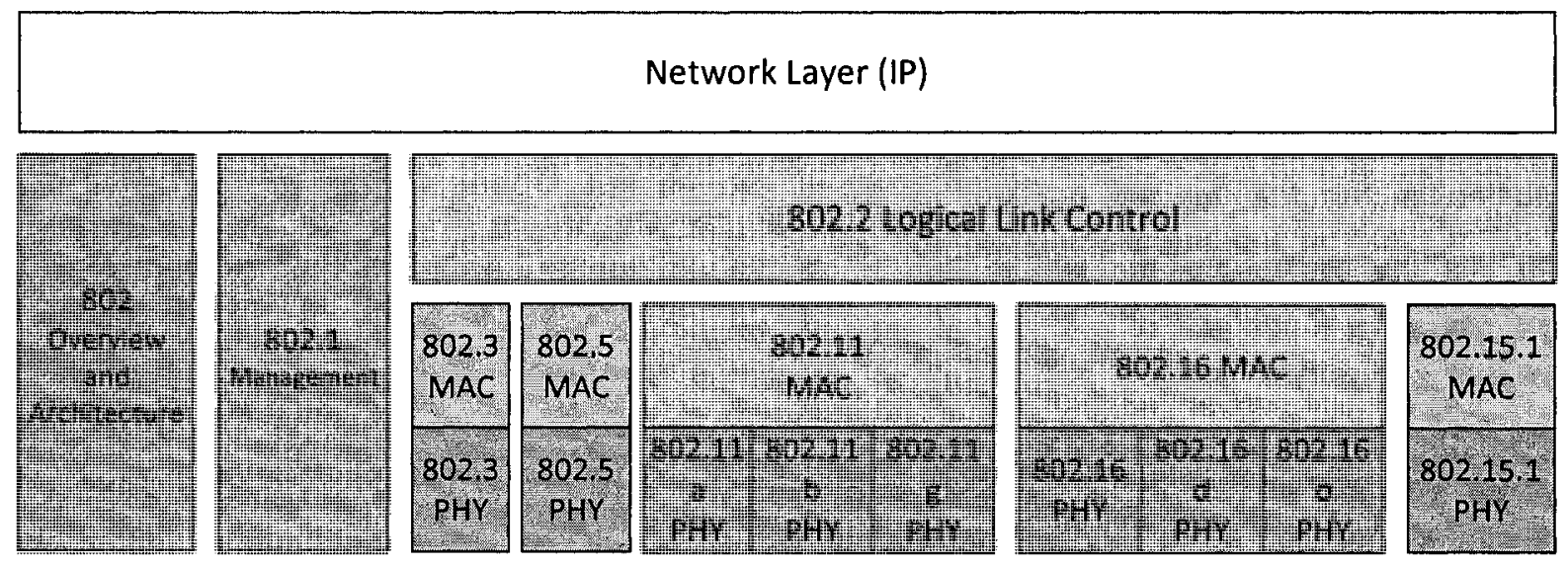

Figure 1.1. IEEE 802 standards

\subsection{IEEE 802 Standards}

The IEEE 802 standards family defines the physical (PHY) and media access (MAC) layer of the 7-layer OSI model. These standards family include the IEEE 802.3 (Wired Ethernet) standard, IEEE 802.1 (management) standard, IEEE 802.5 (token ring) standard, 
very famous IEEE 802.11 (WLAN) and IEEE 802.16 or WiMAX standard. Fig. 1.1 [1] shows the structure of these standards.

\section{○ IEEE 802.11}

It is the most widely deployed wireless LAN standard all over the world. The standard uses variety of modulation techniques and can supports data rates from $1 \mathrm{Mbps}$ up to $54 \mathrm{Mbps}$. The physical layer of the standard is defined as follows:

- IEEE $802.11 \mathrm{~b}$ operates in the $2.4 \mathrm{GHz}$ industrial, scientific and medical (ISM) band. This standard can support data rate up to $11 \mathrm{Mb}$ s and use direct sequence spread spectrum (DSSS) for wireless access [4].

- IEEE 802.11g also operates in the $2.4 \mathrm{GHz}$ industrial, scientific and medical (ISM) band. This standard can support data rate up to $54 \mathrm{Mbps}$ and use orthogonal frequency division multiplexing (OFDM) for wireless access [5].

- IEEE 802.11a operates in the $5 \mathrm{GHz}$ Unlicensed National Information Infrastructure (UNII) band. For wireless access this standard also uses orthogonal frequency division multiplexing (OFDM) and operates at lower transmit power around a maximum of $20 \mathrm{~mW}$ while the $802.11 \mathrm{~g}$ version operates at power maximum of $100 \mathrm{~mW}$. The IEEE 802.11a standard can support data rates up to $54 \mathrm{Mbps}$ [3].

Fig.1.2 shows the logical architecture [2] of the IEEE 802.11 standard where MAC layer is same for all versions of IEEE 802.11 ( $\mathrm{a}, \mathrm{b}$ or $\mathrm{g}$ ). The wireless channel is shared by the users using Carrier Sense Multiple Access with Collision Avoidance (CSMA/CA). The logical link control layer (LLC) serves as an adapter to the higher layers. The service area of this standard is multiple of $3 \mathrm{~m}$ to $30 \mathrm{~m}$. 


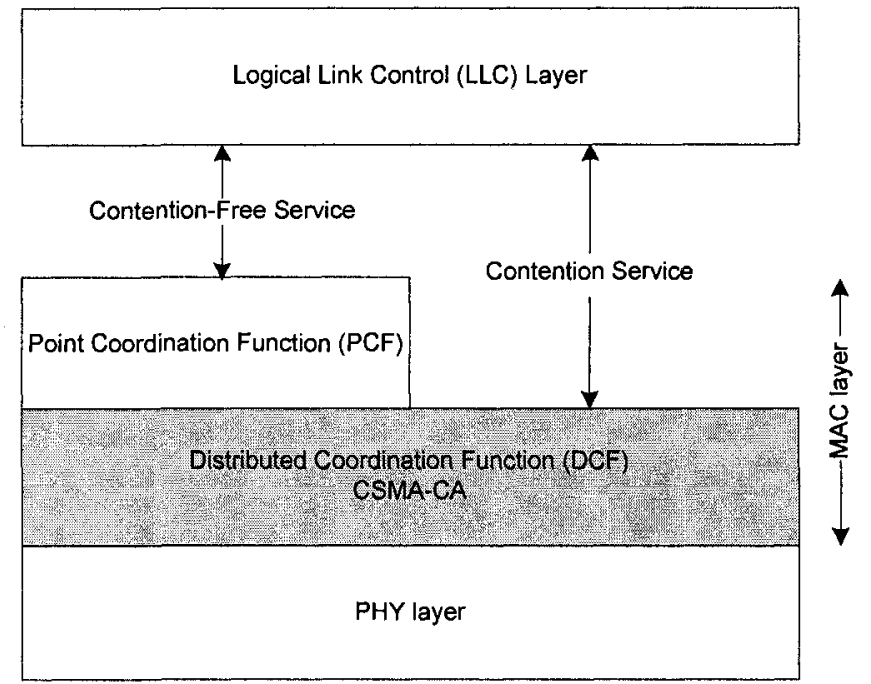

Figure 1.2. IEEE 802.11 logical architecture.

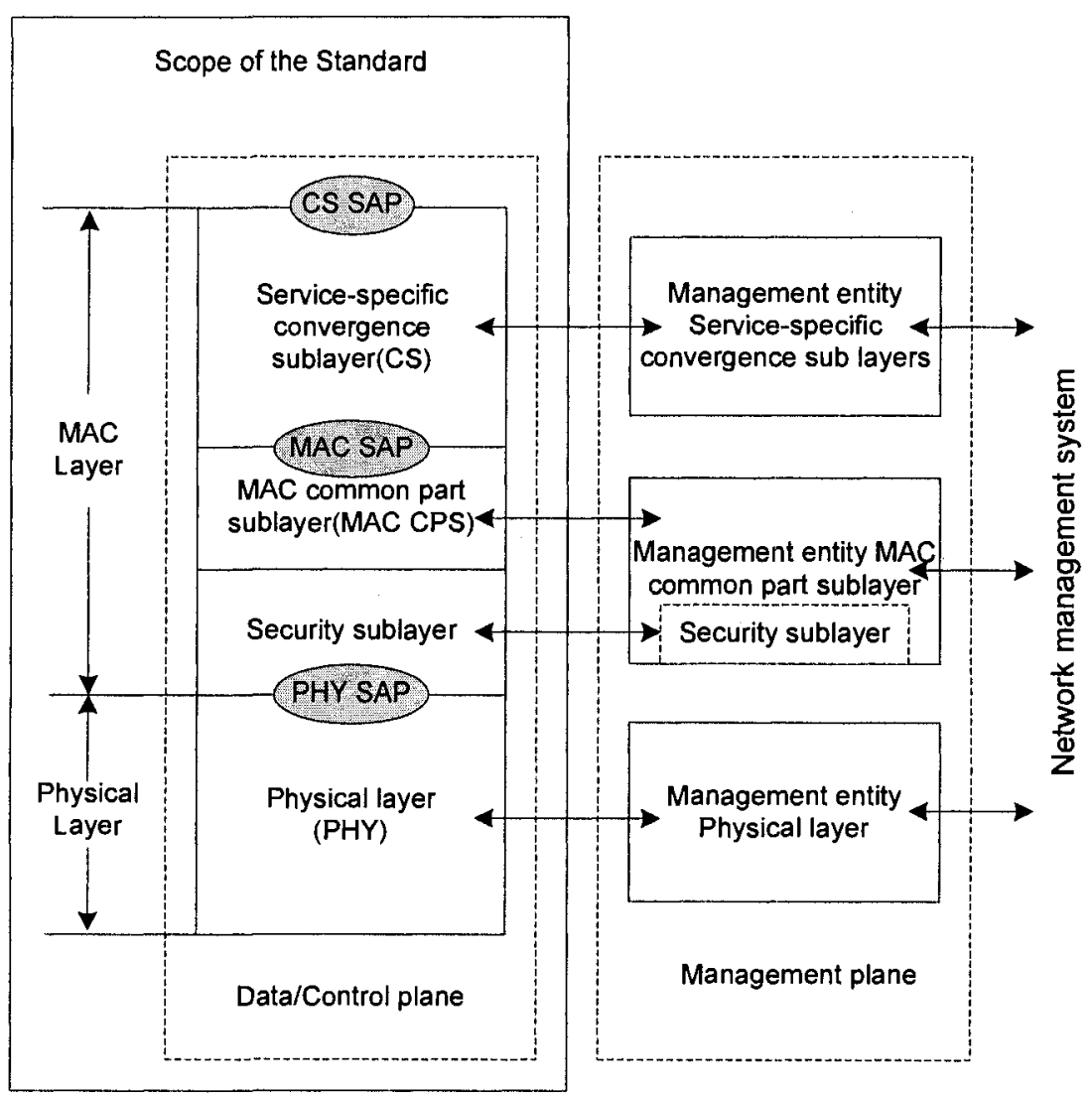

Figure 1.3. IEEE 802.16 protocol stack. 


\section{- WiMAX or IEEE 802.16}

WiMAX standard is well known name of the air interface standard of IEEE 802.16. It is one of the most popular broadband wireless access (BWA) technologies available today which can provides BWA service to fixed, nomadic, portable and mobile stations and enables interoperability and coexistence of BWA systems from different manufactures in a costeffective way. The IEEE 802.16 standard family defines physical layer and MAC layer of 7 layer OSI reference model as show in fig. 1.3. Two variation of WiMAX is currently available: one is based on IEEE 802.16-2004, for fixed wireless application and another is for mobile users based on IEEE 802.16e. Table 1.1 [6] shows a comparison between some of the versions of IEEE 802.16 standards.

Table 1.1. Comparison among IEEE 802.16, 802.16a, 802.16-2004 and 802.16e.

\begin{tabular}{|c|c|c|c|c|}
\hline & $\mathbf{8 0 2 . 1 6}$ & $802.16 a$ & 802.16-2004 & $802.16 \mathrm{e}$ \\
\hline $\begin{array}{l}\text { Frequency of } \\
\text { operation }\end{array}$ & $10-66 \mathrm{GHz}$ & $2-11 \mathrm{GHz}$ & $\begin{array}{lll}2-11 & \mathrm{GHz} & 10-66 \\
\mathrm{GHz} & & \\
\end{array}$ & $2.6 \mathrm{GHz}$ \\
\hline Ch. condition & LOS & NLOS & NLOS & NLOS \\
\hline Bandwidth & 20,25 and $28 \mathrm{MHz}$ & $1.25-28 \mathrm{MHz}$ & $1.25-28 \mathrm{MHz}$ & $1.25-20 \mathrm{MHz}$ \\
\hline $\begin{array}{l}\text { Modulation } \\
\text { Scheme }\end{array}$ & $\begin{array}{l}\text { QPSK, 16QAM and } \\
\text { 64QAM }\end{array}$ & $\begin{array}{lr}\text { OFDM, } & \text { QPSK, } \\
\text { 16QAM } & \text { and } \\
64 Q A M & \\
\end{array}$ & $\begin{array}{lr}\text { OFDM, } & \text { QPSK, } \\
\text { 16QAM } & \text { and } \\
\text { 64QAM } & \\
\end{array}$ & $\begin{array}{lr}\text { OFDM, } & \text { QPSK, } \\
\text { 16QAM } & \text { and } \\
\text { 64QAM } & \\
\end{array}$ \\
\hline $\begin{array}{l}\text { Network } \\
\text { architecture }\end{array}$ & PTP, PMP & PTP, PMP, mesh & PTP, PMP, mesh & PTP, PMP, mesh \\
\hline Bit rate & 32-134 Mbps & $\mathrm{Up}$ to $75 \mathrm{Mbps}$ & $\mathrm{Up}$ to $75 \mathrm{Mbps}$ & $\mathrm{Up}$ to $15 \mathrm{Mbps}$ \\
\hline Mobility & Fixed & Fixed & Fixed & $\begin{array}{l}\text { Pedestrian } \\
\text { Mobility-regional } \\
\text { roaming, maximum } \\
\text { mobility support } \\
125 \mathrm{~km} / \mathrm{h}\end{array}$ \\
\hline $\begin{array}{l}\text { Typical cell } \\
\text { radius }\end{array}$ & 1-3 miles & $\begin{array}{l}\text { Maximum range is } \\
30 \text { miles on the } \\
\text { basis of antenna } \\
\text { height, antenna gain } \\
\text { and transmit power }\end{array}$ & $\begin{array}{l}\text { Maximum range is } \\
30 \text { miles on the } \\
\text { basis of antenna } \\
\text { height, antenna gain } \\
\text { and transmit power }\end{array}$ & $1-3$ miles \\
\hline Applications & $\begin{array}{l}\text { Replacement of } \\
\text { E1/T1 services for } \\
\text { enterprises,backhaul } \\
\text { for hot spots, } \\
\text { residential roadband } \\
\text { access, SOHO } \\
\text { (Small office/home } \\
\text { office) }\end{array}$ & $\begin{array}{l}\text { Alternative to } \\
\text { E1/T1, DSL, cable } \\
\text { backhaul for cellular } \\
\text { and WiFi, VoIP, } \\
\text { Internet connections }\end{array}$ & $\begin{array}{l}801.16 \text { plus } 802.16 \mathrm{a} \\
\text { applications }\end{array}$ & $\begin{array}{l}802.16-2004 \\
\text { applications plus } \\
\text { fixed VoIP QoS- } \\
\text { based applications, } \\
\text { and enterprise } \\
\text { networking }\end{array}$ \\
\hline
\end{tabular}




\section{○ IEEE 802.15}

The IEEE 802.15 standard focus on WPANs with ranges only in the order of 10 feet. Despite of this short service range network using this standard is increasingly prevalent as wireless networking evolves to support a variety of platforms, including those in the home such as household applications. As for example mobile phones enabled with IEEE 802.15 technologies benefit from ability to connect to other phones, computers or devices [1].

\subsection{HiperLAN/2 Standard}

For providing wireless connectivity in domestic premises as well as business premises as for example apartments, small house, large hall room, office, hospital, university, shopping mall, airport, etc (Fig. 1.4). Broadband Radio Access Networks (BRAN) division of European Telecommunications Standards Institute (ETSI) developed high performance wireless LAN standard known as High Performance Radio Local Area Network (HiperLAN/2) [7]. It is analogous to the IEEE 802.11 standard and considered as superior in terms of throughput and design perspective.

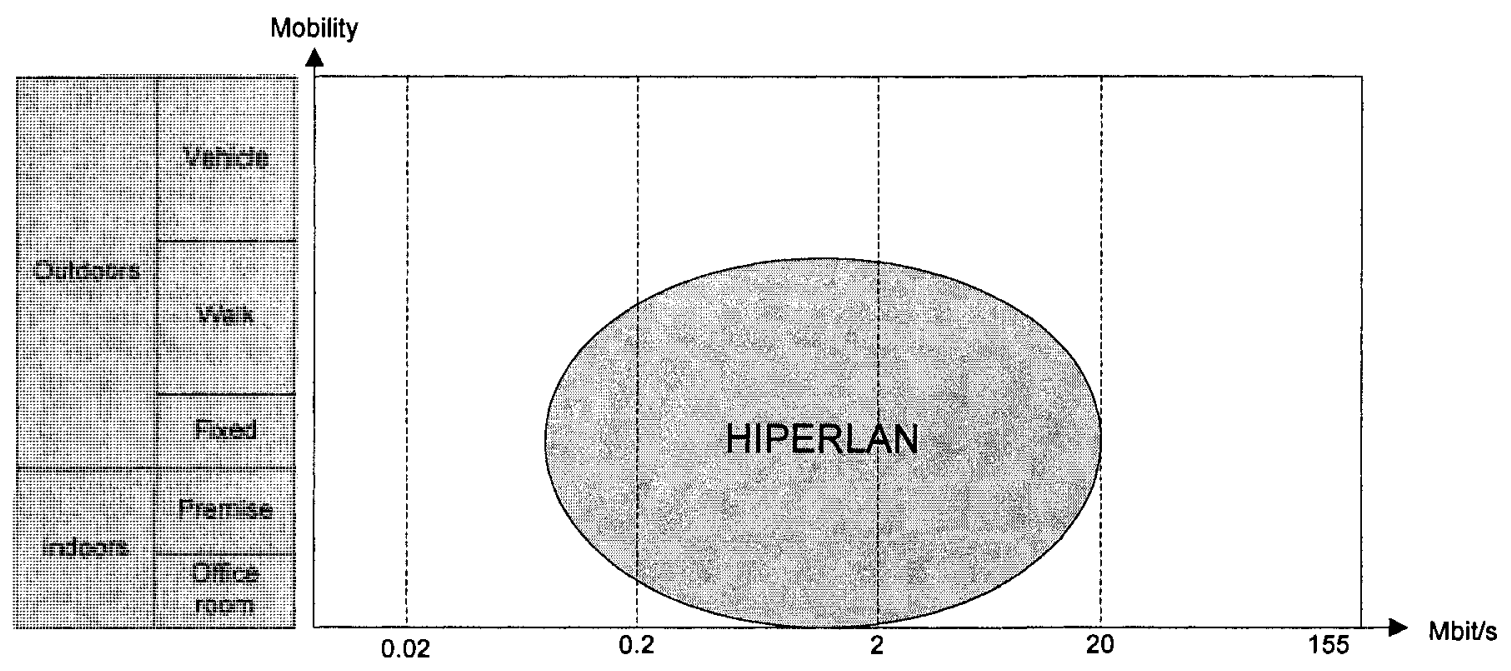

Figure 1.4. HiperLAN operation conditions. 


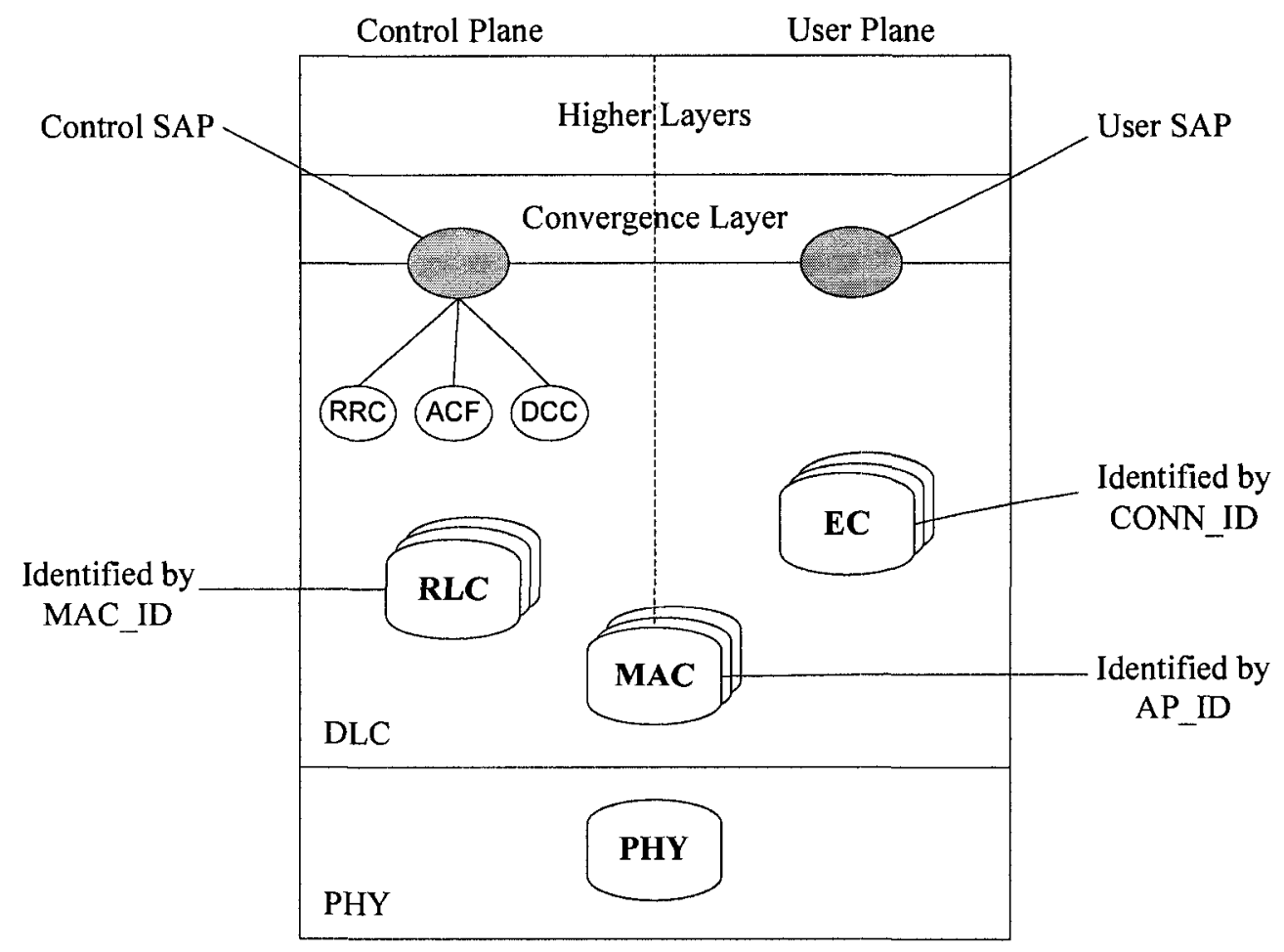

Figure 1.5. HiperLAN/2 protocol stack and functions.

The HiperLAN type 2 standard defines the Physical layer and the Data link layer of 7 layer OSI reference model. In the standard Convergence Layer (CL), Data Link Control layer (DLC) and Physical Layer are defined in details (Fig. 1.5) [7]. Here the Convergence Layer performs as an adapter for DLC and Physical layer to the higher layer and makes the system able to provide wide range of service such as IP, ATM and UMTS. An H/2 system can provide flexible service to hand held devices, computers, laptops or other wireless equipments. The standard operates at $5 \mathrm{Ghz}$ band and can offers data rates from $6 \mathrm{Mbps}$ up to $54 \mathrm{Mbps}$. It uses orthogonal frequency division multiplexing (OFDM) in its physical layer and dynamic TDMA TDD based protocol in MAC layer.

Physical layer of HiperLAN/2 system uses OFDM to cope with fading effect. Each OFDM symbol contains 52 subcarriers around one of the possible carriers (Table 2.7) where 48 subcarriers are data carrier and 4 are pilot carriers. Data received from data link layer is 
used to modulate the subcarriers using 7 different types of modulation techniques depending of channel condition. A particular modulation technique is chosen to achieve highest data rate for the erroneous channel.

To deploy HiperLAN/2 system total coverage area is divided into cells. Each cell is served by an Access Point. Each cell operates in a frequency that is different from neighbouring Access Points. Mobile nodes choose Access Point for association, depending on signal strength and it is known as dynamic frequency selection (DFS). Mobile terminals can move from one cell to another. Network uses roaming procedure to provide services to the mobile terminal. A central controller supervises this roaming procedure.

According to the HiperLAN/2 standard [7] network can be setup in two ways: one in infrastructure mode and another in ad-hoc mode. In infrastructure mode mobile terminals exchange their data through the Access Point. On the other hand in ad-hoc mode mobile terminals communicate directly under supervision of Access Point. Inside a cell, mobile node gets access of resource using dynamic TDMA. To serve different types of data and ensure their QoS, the standard defines two types of resource allocation services for time bounded traffic and delay insensitive traffic. For delay sensitive traffic while setting up connection, mobile nodes negotiate with the Access Point for fixed capacity assignment (FCA). In FCA mobile nodes get certain amount of time assignment in each time slot. But for delay insensitive traffic such as 'data traffic' Access Point distributes available bandwidth among the mobile nodes using some preset procedure. Here mobile terminals (MT) put their service request in the specific predefined time slot known as contention period of the time frame, if the mobile terminal is not associated with the Access Point. The mobile terminals can also put its service request at the end of its transmission to the Access Point (AP) in uplink phase. The 
AP grants the service request of the mobile terminals using parameters received through the service request and other rules set for the allocation purpose. APs use certain portion of the time frame to inform the status of the service requests to the MT. In this feedback message AP also inform the mobile terminals the specific time and the length of time, i.e. when and how long it will receive or able to transmit data. Remaining part of the time frame is used by the mobile terminals to exchange data between them or with Access Point in uplink or downlink slots.

\subsection{Motivation}

It is the function of MAC layer of an Access Point to divide available transmission time among the mobile nodes and build a TDMA/TDD frame. To do this job Access Points have to use a set of rules. But the HiperLAN/2 standard does not define any protocol for this purpose. In this thesis we proposed two protocols for resource allocation purpose and evaluated their performance for a dynamically modulated interconnected WLAN.

We also found that very few studies had been done to evaluate the capability of HiperLAN/2 deliver different kinds of traffic. Among the studies, optimum number of random access slots for different kinds of traffic to meet certain QoS requirement is studied in [11]-[14]. In the HiperLAN/2 standard optimum number of resource allocation phases in a TDMA \TDD frame is not specified and in [11]-[14] authors treats this deficiency. In [15][19] capability of HiperLAN/2 is evaluated to serve different kinds of traffic using well known resource allocation schemes such as round robin, minimum overhead Round Robin (MORR) and First In First Out (FIFO) schemes. But in these studies the condition of buffers in the mobile terminals was not examined and also these studies were performed only for error free channel and with stationary mobile terminals for either in uplink or in downlink phase. But in 
our study we have considered erroneous channel and dynamic modulation for both uplink and downlink channel.

\subsection{Thesis objective}

Packet loss can occur in the network during transmission and also in the buffer due to overflow as buffer size is fixed. Packet loss in the buffer can be minimized using resource allocation i.e., providing transmission opportunity to the user. In this thesis our objective is to investigate performance of three resource allocation schemes namely Minimum Overhead Round Robin (MORR), which does not depend on user's buffer condition, Weighted Minimum Overhead Round Robin (WMORR) which is a function of user buffer as well as the waiting time for transmission opportunity and Weighted Round Robin (WRR) which is a function of user buffer only. These schemes are used for resource allocation to the users of the WLANs deployed in the metropolitan area using the HiperLAN type 2 standard. For this purpose we design WLAN system where user can move from one place to another inside the WLANs and user rates are dynamically adjusted based on their distance from the Access Points. We develop generic simulation software for the network and use it for the three resource allocation policies. To evaluate performance of the allocation schemes we used average buffer occupancy, packet delivery time, packet drop probability, overhead in downlink and uplink phases.

\subsection{Thesis organization}

The thesis is organized as follows: chapter 2 describes physical layer and MAC layer of $\mathrm{H} / 2$ system. The proposed three resource allocation schemes and simulation setup are described in chapter 3 and 4 respectively. Simulation results are presented in chapter 5. Finally conclusion and suggestion for future work are presented in chapter 6 . 


\section{Chapter 2:H/2 MAC \& Physical Layer}

\subsection{MAC Layer}

HiperLAN/2 standard use TDMA/TDD based MAC protocol [8]. Duration of a MAC frame is $2 \mathrm{~ms}$. This $2 \mathrm{~ms}$ time frame is further divided into a number of short time phases to accommodate broadcast phase, uplink (data direction MT to AP) phase, downlink (data direction $\mathrm{AP}$ to $\mathrm{MT}$ ) phase, direct link (data exchange between two MT directly) phase and random access phase. Data exchanges between MTs can occur in two ways: through AP in infrastructure mode or directly between MTs supervised by AP in Ad-hoc mode. In the infrastructure five variable length phases make up the MAC frame (Fig. 2.1). These short time phases are described below.

\section{- Broadcast phase}

In this part of the MAC frame, time to receive data, assigned transmission opportunity, amount of receivable data, time at which a particular MT should transmit and other control information is announced by the $\mathrm{AP}$ using three $\mathrm{BCH}, \mathrm{FCH}$ and $\mathrm{ACH}$ broadcast transport channels (Fig. 2.1).

- $\mathrm{BCH}$ (Broadcast CHannel): Different control channel information of the whole cell is broadcasted by $\mathrm{AP}$ using $\mathrm{BCH}$ 
- FCH (Frame CHannel): In this short phase AP inform MTs about time to receive or transmit, total allocated time for receive or transmit data and selected physical mode for uplink or downlink.

- $\mathrm{ACH}$ (Access Feedback CHannel): Results of service request by MTs in $\mathrm{RCH}$ phase of previous MAC frame is announced by $\mathrm{AP}$ using $\mathrm{ACH}$

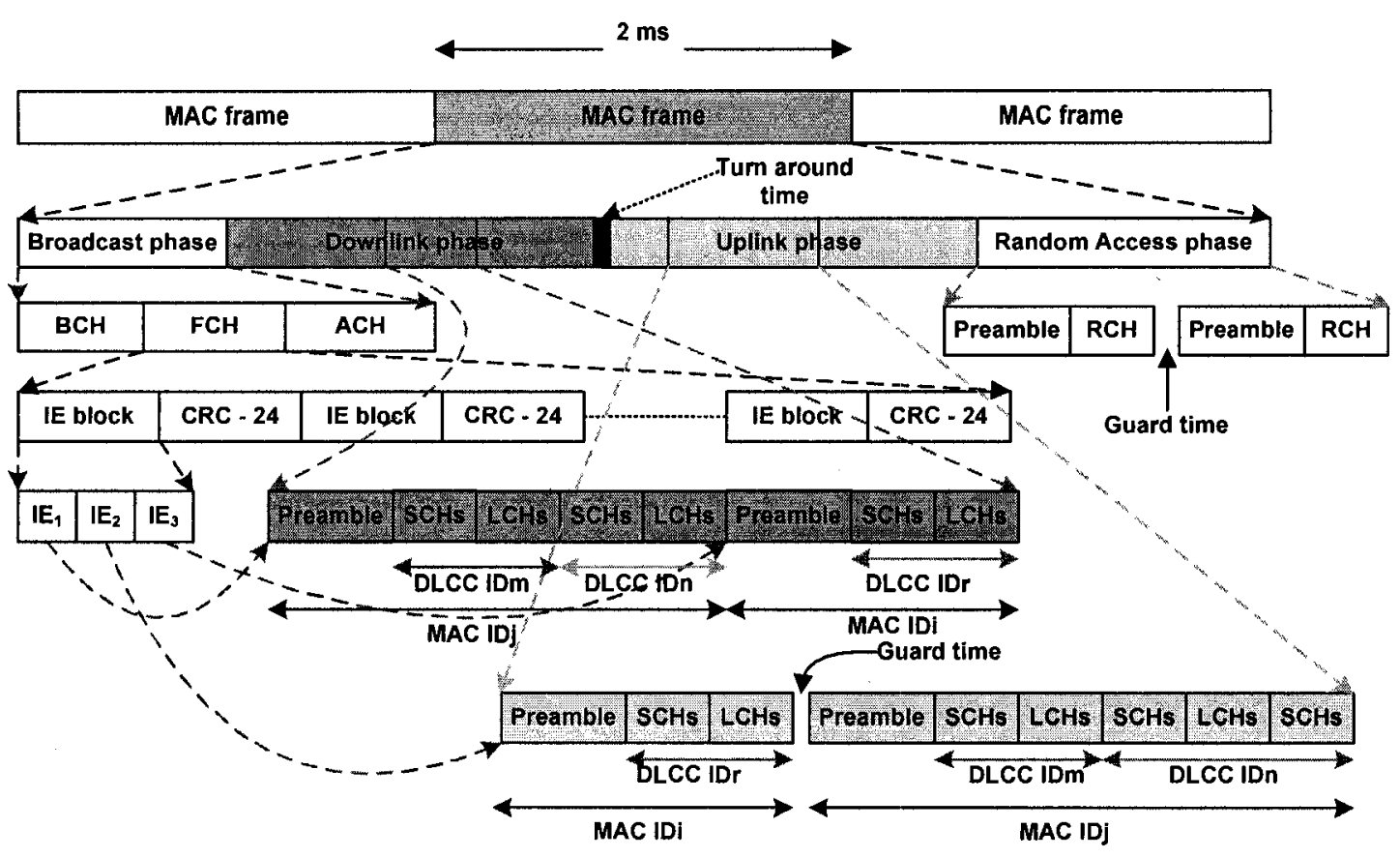

Figure 2.1. MAC frame structure.

\section{- Downlink Phase (DL)}

Protocol Data Units (PDU) of AP destinated to MTs form this phase. A stream of PDUs transmitted by AP for a MT consists of a preamble followed by number of Long transport Channels (LCH) and Short transport Channels (SCH) (Fig. 2.1). LCHs contain data for MT while SCH contains ARQ and other control information. AP combine all the LCHs and SCHs scheduled for transmission and use one uplink burst for transmission to a MT. 


\section{- Uplink Phase (UL)}

MTs when polled as per the FCH channel transmit their uplink data in this phase. Uplink burst contains (Fig. 2.1) number of LCH PDUs for data transmission and/or SCH PDUs for acknowledgement, resource request and various control messages. MT combine all the LCHs and SCHs scheduled for transmission and use one uplink burst for transmission to the AP.

\section{- Random Access phase}

A Slotted-ALHOA based contention is used in this short period. Collision may occur in this slot. To control access to the $\mathrm{RCH}$ phase a contention window $\mathrm{CW}_{\mathrm{a}}$ is maintained in each MT. The size of contention window derived using the number $a$, where $a$ is the number of retransmission attempts made by the MT. For the first access the value of $a$ is set to 0 . The size of $\mathrm{CW}_{\mathrm{a}}$ is defined as below:

Initial Attempt: $a=0, C W_{a}=n$

$$
\text { Retransmission : } a \geq 1, C W_{a}=\left\{\begin{array}{cc}
256 & 2^{a} \geq 256 \\
2^{a} & n<2^{a} \leq 256 \\
n & n \geq 2^{a}
\end{array}\right.
$$

Here, $\mathrm{n}$ is the number of $\mathrm{RCH}$ in the MAC frame. The $\mathrm{RCH}$ used for $\mathrm{a}^{\text {th }}$ transmission is chosen by a uniformly distributed random value $r_{a}$ within the interval $\left[1, C_{2}\right]$. To determine the $\mathrm{RCH}$, when $\mathrm{a}=0$ the $\mathrm{MT}$ start counting from the first $\mathrm{RCH}$ of the current MAC frame. But in the case of retransmission MT starts counting from the MAC frame in which it gets information about failure in the previous transmission event. When the $\mathrm{RCH}$ number equals $r_{a}, M T$ transmits resource request. 


\subsection{Physical Layer}

In the $\mathrm{H} / 2$ standard, the physical layer [20] is based on Orthogonal Frequency Division Multiplexing (OFDM) as shown in fig. 2.2. OFDM causes physical bursts to perform better with interference such as frequency selective fading and burst errors caused by wideband fading channel.

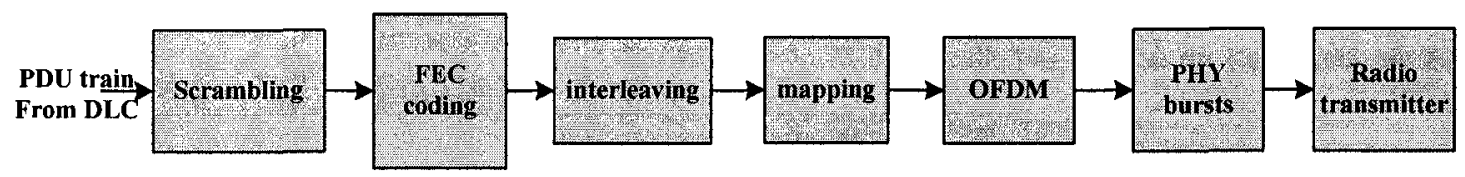

Figure 2.2. Block diagram of HiperLAN/2 physical layer.

Data supplied to the physical layer is scrambled with a length-127 scrambler (Fig. 2.3). In the scrambler, a generator polynomial (equation 2.1) is used [20]. Receiver side uses the same scrambler to descramble the data.

$$
S(x)=x^{7}+x^{4}+1
$$

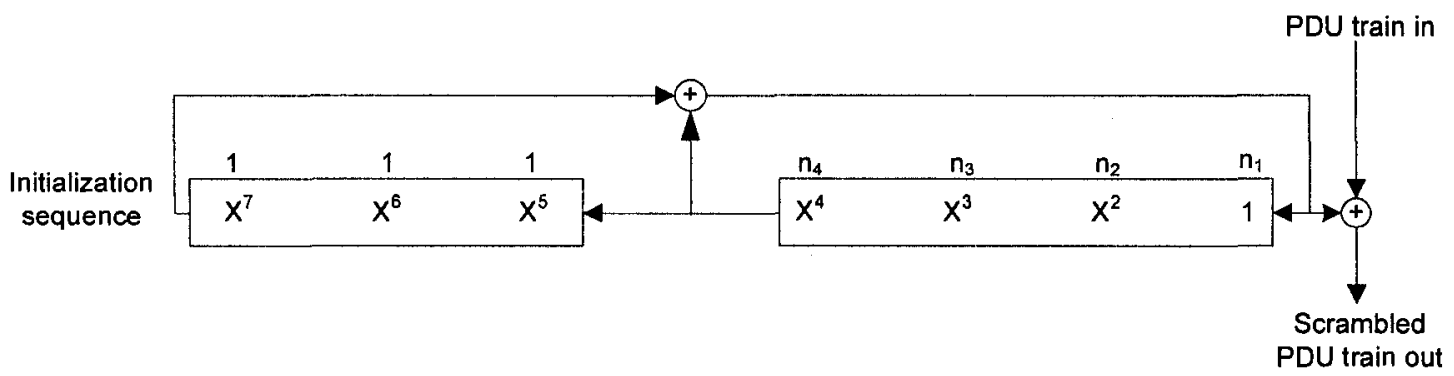

Figure 2.3. HiperLAN/2 scambler.

The scrambled data is encoded with a $1 / 2$ rate convolutional encoder followed by puncturing. Using Puncturing, $1 / 2,3 / 4$ or $9 / 16$ data rates are achieved. This coded data is passed through an interleave stage to combat burst channel error.

Improvement of radio link capability in different kinds of channel interferences are achieved by using one of the 7 modulation schemes dynamically as in table 2.1 [20]. 
Appropriate data rate is assigned by $\mathrm{AP}$ depending on the channel condition. Data from Interleave stage is divided into groups of 1, 2, 4 or 6 bits. Each group is converted into complex numbers representing BPSK, QPSK, 16QAM or 64QAM constellation points (Fig. 2.4). Gray coding is used to map the data bits into the constellation points. A normalization factor $\mathrm{K}_{\mathrm{MOD}}$ is multiplied with the complex valued output of the mapper to get the final output from this stage as shown in table 2.2 and table 2.3. A PDU train can have PDUs modulated by different types of modulation but inside a particular PDU only one modulation type is used.

Table 2.1. H/2 PHYSICAL LAYER MODES.

\begin{tabular}{c|c|c|c|c|c|c}
\hline Mode & Modulation & $\begin{array}{c}\text { Code } \\
\text { rate }\end{array}$ & $\begin{array}{c}\text { Nominal bit } \\
\text { rate (Mb/s) }\end{array}$ & $\begin{array}{c}\text { Coded bits per } \\
\text { sub-carrier }\end{array}$ & $\begin{array}{c}\text { Coded bits per } \\
\text { OFDM symbol }\end{array}$ & $\begin{array}{c}\text { Data bits per } \\
\text { OFDM symbol }\end{array}$ \\
\hline 1 & BPSK & $1 / 2$ & 6 & 1 & 48 & 24 \\
\hline 2 & BPSK & $3 / 4$ & 9 & 1 & 48 & 36 \\
\hline 3 & QPSK & $1 / 2$ & 12 & 2 & 96 & 48 \\
\hline 4 & QPSK & $3 / 4$ & 18 & 2 & 96 & 72 \\
\hline 5 & 16 QAM & $9 / 16$ & 27 & 4 & 192 & 108 \\
\hline 6 & 16 QAM & $3 / 4$ & 36 & 4 & 192 & 144 \\
\hline 7 & 64 QAM & $3 / 4$ & 54 & 6 & 288 & 216 \\
\hline
\end{tabular}

Table 2.2. MODULATION DEPENDENT NORMALIZATION FACTOR $\mathrm{K}_{\text {MOD }}$

\begin{tabular}{l|l}
\hline Modulation & $\mathbf{K}_{\text {MOD }}$ \\
\hline BPSK & 1 \\
\hline QPSK & $1 / \sqrt{2}$ \\
\hline 16QAM & $1 / \sqrt{10}$ \\
\hline 64QAM & $1 / \sqrt{42}$ \\
\hline
\end{tabular}


Table 2.3. ENCODING TABLES FOR BPSK, QPSK, 16QAM AND 64QAM.

\begin{tabular}{c|c|c}
\hline \multicolumn{3}{c}{ BPSK } \\
\hline Input bit $\mathrm{b}_{1}$ & $\mathrm{I}$-out & Q-out \\
\hline 0 & -1 & 0 \\
\hline 1 & 1 & 0 \\
\hline
\end{tabular}

\begin{tabular}{c|c|c|c}
\hline \multicolumn{4}{|c}{ QPSK } \\
\hline Input bit $b_{1}$ & I-out & Input bit $b_{2}$ & Q-out \\
\hline 0 & -1 & 0 & -1 \\
\hline 1 & 1 & 1 & 1 \\
\hline
\end{tabular}

\begin{tabular}{c|c|c|c}
\hline \multicolumn{4}{|c}{ 16QAM } \\
\hline Input bit $b_{1} b_{2}$ & I-out & Input bit $b_{3} b_{4}$ & Q-out \\
\hline 00 & -3 & 00 & -3 \\
\hline 01 & -1 & 01 & -1 \\
\hline 11 & 1 & 11 & 1 \\
\hline 10 & 3 & 10 & 3 \\
\hline
\end{tabular}

\begin{tabular}{c|c|c|c}
\hline \multicolumn{5}{|c}{ 64QAM } \\
\hline Input bit $\mathrm{b}_{1} \mathrm{~b}_{2} \mathrm{~b}_{3}$ & $\mathrm{I}$-out & Input bit $\mathrm{b}_{4} \mathrm{~b}_{5} \mathrm{~b}_{6}$ & Q-out \\
\hline 000 & -7 & 000 & -7 \\
\hline 001 & -5 & 001 & -5 \\
\hline 011 & -3 & 011 & -3 \\
\hline 010 & -1 & 010 & -1 \\
\hline 110 & 1 & 110 & 1 \\
\hline 111 & 3 & 111 & 3 \\
\hline 101 & 5 & 101 & 5 \\
\hline 100 & 7 & 100 & 7 \\
\hline
\end{tabular}



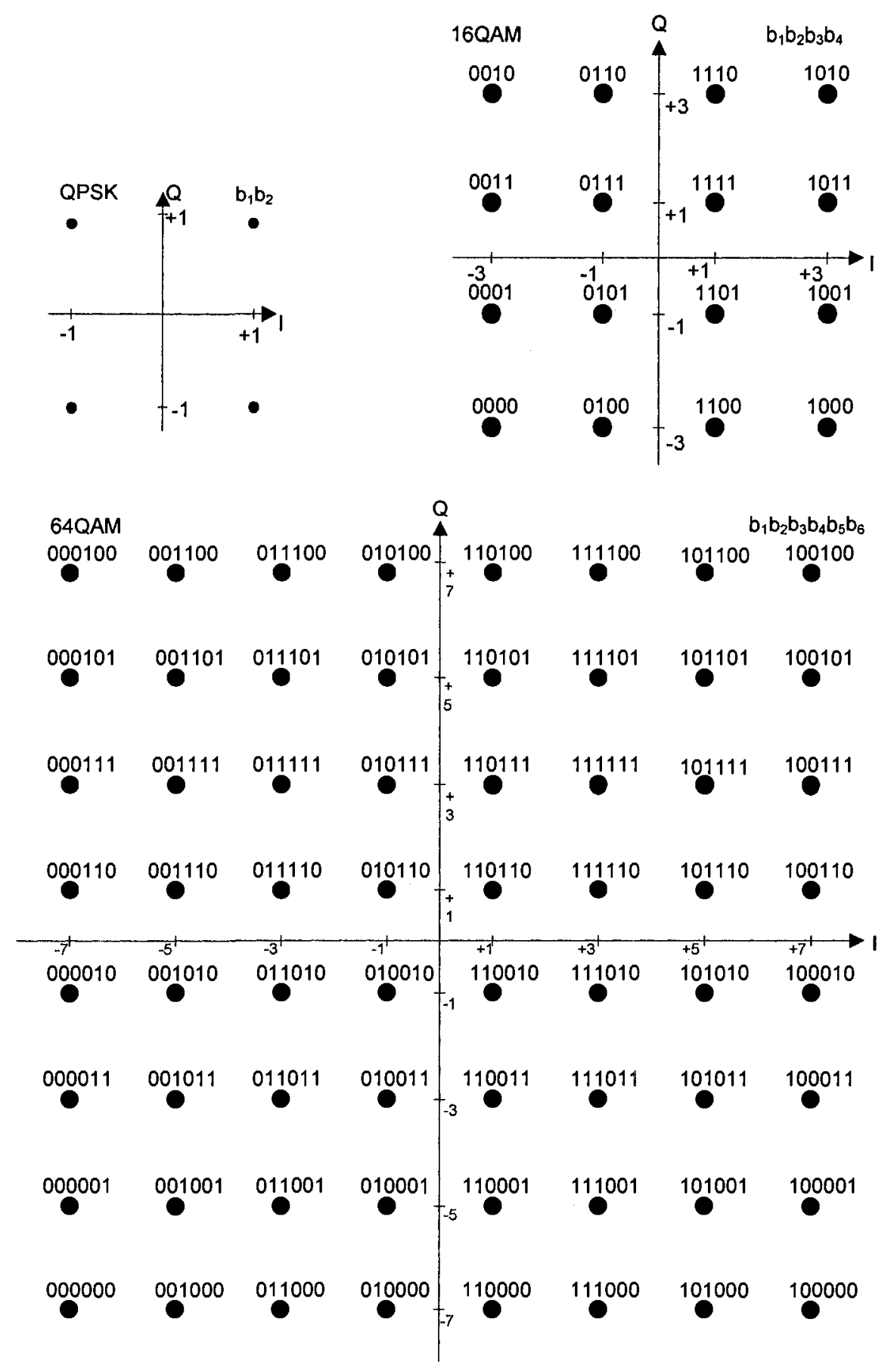

Figure 2.4. H/2 physical layer BPSK, QPSK, 16QAM or 64QAM constellation points.

Complex valued output of the selected mapper is divided into groups to be transmited by 48 subcarriers of an OFDM symbol. 4 pilot carriers are added with the 48 subcarriers to complete the OFDM symbol of 52 subcarriers. A certain amount of the useful part of the 
OFDM symbol, known as cyclic prefix is repeated at the beginning to form the final symbol to prevent inter symbol interference (ISI) and inter-carrier interference (ICI). Numerical values of OFDM parameters [20] used in the $\mathrm{H} / 2$ are presented in table 2.4.

Table 2.4. OPDM PARAMETERS.

\begin{tabular}{l|l}
\hline \multicolumn{1}{c|}{ Parameter } & \multicolumn{1}{c}{ Value } \\
\hline Sapling rate $\left(\mathrm{f}_{\mathrm{s}}\right)$ & $20 \mathrm{MHz}$ \\
\hline Useful symbol duration $\left(\mathrm{T}_{\mathrm{u}}\right)$ & $3.2 \mu \mathrm{s}$ \\
\hline Cyclic prefix duration $\left(\mathrm{T}_{\mathrm{cp}}\right)$ & 0.8 \\
\hline Total symbol duration $\left(\mathrm{T}_{\mathrm{Total}}\right)$ & 4.0 \\
\hline Number of data subcarrier & 48 \\
\hline Number of pilot subcarrier & 4 \\
\hline FFT size & 64 \\
\hline Subcarrier spacing $(\Delta \mathrm{f})$ & $0.3125 \mathrm{MHz}$ \\
\hline Total bandwidth & $16.875 \mathrm{MHz}$ \\
\hline
\end{tabular}

\section{- Frequency Band}

Regulatory bodies such as FCC in US and CEPT in Europe specified frequency band to setup wireless systems using $\mathrm{H} / 2$ standard. According to the decision made by CEPT, to build Public or Private system using $\mathrm{H} / 2$ standard, deployment should be in $5 \mathrm{GHz}$ frequency band as summarized in Table 2.5 [1].

Table 2.5. H/2 OPERATING CONDITIONS.

\begin{tabular}{|c|c|c|}
\hline Frequency band & RF Power limit & Comments \\
\hline $\begin{array}{l}5150 \mathrm{MHz} \text { to } 5350 \\
\mathrm{MHz}\end{array}$ & $200 \mathrm{~mW}$ mean EIRP & $\begin{array}{l}\text { Indoor use only and implementation of Dynamic } \\
\text { Frequency Selection (DFS) and Transmit Power } \\
\text { Control (TPC) }\end{array}$ \\
\hline $\begin{array}{llll}5470 & \mathrm{MHz} \text { to } & 5725 \\
\mathrm{MHz} & & & \\
\end{array}$ & $1 \mathrm{~W}$ mean EIRP & $\begin{array}{l}\text { Indoor and outdoor use implementation of DFS and } \\
\text { TPC. }\end{array}$ \\
\hline
\end{tabular}

\section{- Channel model}

According to the standard HiperLAN/2, 5 channel models [23] are defined (table 2.6) during the standardization process to setup WLAN in domestic premises as well as business premises to cover areas such as apartments, small house, large hall room, office, hospital, university, shopping mall, airport, etc. The defined channels represent a range of different 
noise and interference condition in different deployment scenario. We used Channel model E in our simulation, as it is designed for outdoor environment and NLOS.

Table 2.6. H/2 CHANNEL MODELS.

\begin{tabular}{c|c|c|c}
\hline Name & $\begin{array}{c}\text { RMS delay } \\
\text { spread }\end{array}$ & Characteristic & Environment \\
\hline $\mathrm{A}$ & $50 \mathrm{~ns}$ & $\begin{array}{c}\text { Narrowband } \\
\text { Rayleigh }\end{array}$ & $\begin{array}{c}\text { Residential } \\
\text { NLOS }\end{array}$ \\
\hline $\mathrm{B}$ & $100 \mathrm{~ns}$ & $\begin{array}{c}\text { Wideband } \\
\text { Rayleigh }\end{array}$ & $\begin{array}{c}\text { Small Office } \\
\text { NLOS }\end{array}$ \\
\hline $\mathrm{C}$ & $150 \mathrm{~ns}$ & $\begin{array}{c}\text { Wideband } \\
\text { Rayleigh }\end{array}$ & $\begin{array}{c}\text { Large Office } \\
\text { NLOS }\end{array}$ \\
\hline $\mathrm{D}$ & $140 \mathrm{~ns}$ & $\begin{array}{c}\text { Wideband } \\
\text { Rician }\end{array}$ & $\begin{array}{c}\text { Large Office } \\
\text { LOS }\end{array}$ \\
\hline E & $250 \mathrm{~ns}$ & $\begin{array}{c}\text { Wideband } \\
\text { Rayleigh }\end{array}$ & $\begin{array}{c}\text { Outdoor } \\
\text { NLOS }\end{array}$ \\
\hline
\end{tabular}

\section{- Radio transmission: RF carrier}

Two frequency bands are defined in HiperLAN/2 standard [20]. One is named as lower frequency band occupying $5150 \mathrm{MHz}$ to $5350 \mathrm{MHz}$ and the other one is upper frequency band between $5470 \mathrm{MHz}$ to $5725 \mathrm{MHz}$. The carrier number $\mathrm{n}_{\text {carrier }}$ is defined as in equation 2.2 .

$$
n_{\text {carrier }}=\frac{f_{c}-5000 \mathrm{MHz}}{5 \mathrm{MHz}}
$$

Carrier frequency are spaced $20 \mathrm{MHz}$ apart. All transmission is centered on one of the nominal frequencies. According to the standard the centre frequencies in Europe are as in table 2.7. 
Table 2.7. NOMINAL CARRIER FREQUENCY ALLOCATION TABLE FOR H/2 IN EUROPE.

\begin{tabular}{c|c|c|c}
\hline $\mathbf{n}_{\text {carrier }}$ & Band & $\mathbf{f}_{\mathbf{c}}[\mathbf{M H z}]$ & Mean EIRP[dBm] \\
\hline 36 & Lower & 5180 & 23 \\
\hline 40 & Lower & 5200 & 23 \\
\hline 44 & Lower & 5220 & 23 \\
\hline 48 & lower & 5240 & 23 \\
\hline 52 & lower & 5260 & 23 \\
\hline 56 & lower & 5280 & 23 \\
\hline 60 & lower & 5300 & 23 \\
\hline 64 & lower & 5320 & 23 \\
\hline 100 & upper & 5500 & 30 \\
\hline 104 & upper & 5520 & 30 \\
\hline 108 & upper & 5540 & 30 \\
\hline 112 & upper & 5560 & 30 \\
\hline 116 & upper & 5580 & 30 \\
\hline 120 & upper & 5600 & 30 \\
\hline 124 & upper & 5620 & 30 \\
\hline 128 & upper & 5640 & 30 \\
\hline 132 & upper & 5660 & 30 \\
\hline 136 & upper & 5680 & 30 \\
\hline 140 & upper & 5700 & 23 \\
\hline
\end{tabular}




\section{Chapter 3: Resource allocation policies}

One of the main functions of an AP is to organize the TDMA frame. Efficiency of the system depends on the allocation mechanism adopted by the AP. QoS provided by MT also depends on the allocation mechanism of AP. We simulate the system for three allocation schemes.

\subsection{Minimum Overhead Round Robin (MORR)}

In this scheme AP allocates equal capacity share to all active MTs. Whenever one MT is given transmission opportunity, it gets the opportunity to transmit up to the system capacity. If the MT does not have enough packets in the buffer, frame is filled by packets of another MT. A certain MT is visited again when AP has completed its visit to all other MTs. This process helps to reduce overhead of the system. Fig. 3.1 shows a flowchart for this scheme.

\subsection{Weighted Minimum Overhead Round Robin (WMORR)}

Resource request of MT contains it's buffer condition and AP has the information in regard to the elapsed time since last transmission opportunity the MT has got. Using product of this two information an allocation mechanism is designed to give transmission opportunity to a MT waiting for long and that has highest number of packets in buffer. It is termed as MORR because whenever a MT is visited capacity is assigned up to the capacity of the network. Mathematically the buffer status and waiting time gives a matrix $M$ (equation 3.1). The AP compares and combines all such matrix of all MT and hence decides upon the 
capacity allocation to each MT. Basically the time or capacity assigned to $i^{\text {th }}$ MT is given by equation 3.2. Flowchart of this scheme is presented in Fig. 3.2.

$$
\begin{gathered}
M_{i}=B_{M T_{i}} \times W_{M T_{i}} \\
T_{M T_{i}}=T_{\text {Frame }} \frac{M_{i}}{\sum_{\mathrm{j}=1}^{N} M_{j}}
\end{gathered}
$$

Where,

$$
\begin{aligned}
& B_{M T_{i}} \quad=\text { No. of PDUs in the buffer of } \mathrm{MT}_{\mathrm{i}} \cdot \\
& W_{M T_{i}} \quad=\text { Waiting time for transmission opportunity of } \mathrm{MT}_{\mathrm{i}} \cdot \\
& \mathrm{M}_{\mathrm{i}} \quad=\text { Weight of } \mathrm{MT}_{\mathrm{i} \cdot} \\
& \mathrm{N} \quad=\text { Total no. of MTs associated with AP. } \\
& \mathrm{T}_{\text {Framae }} \quad=\text { Total Uplink or Downlink time in a TDMA frame. } \\
& T_{M T_{i}} \quad=\text { Total downlink or uplink time allocated to MT }
\end{aligned}
$$




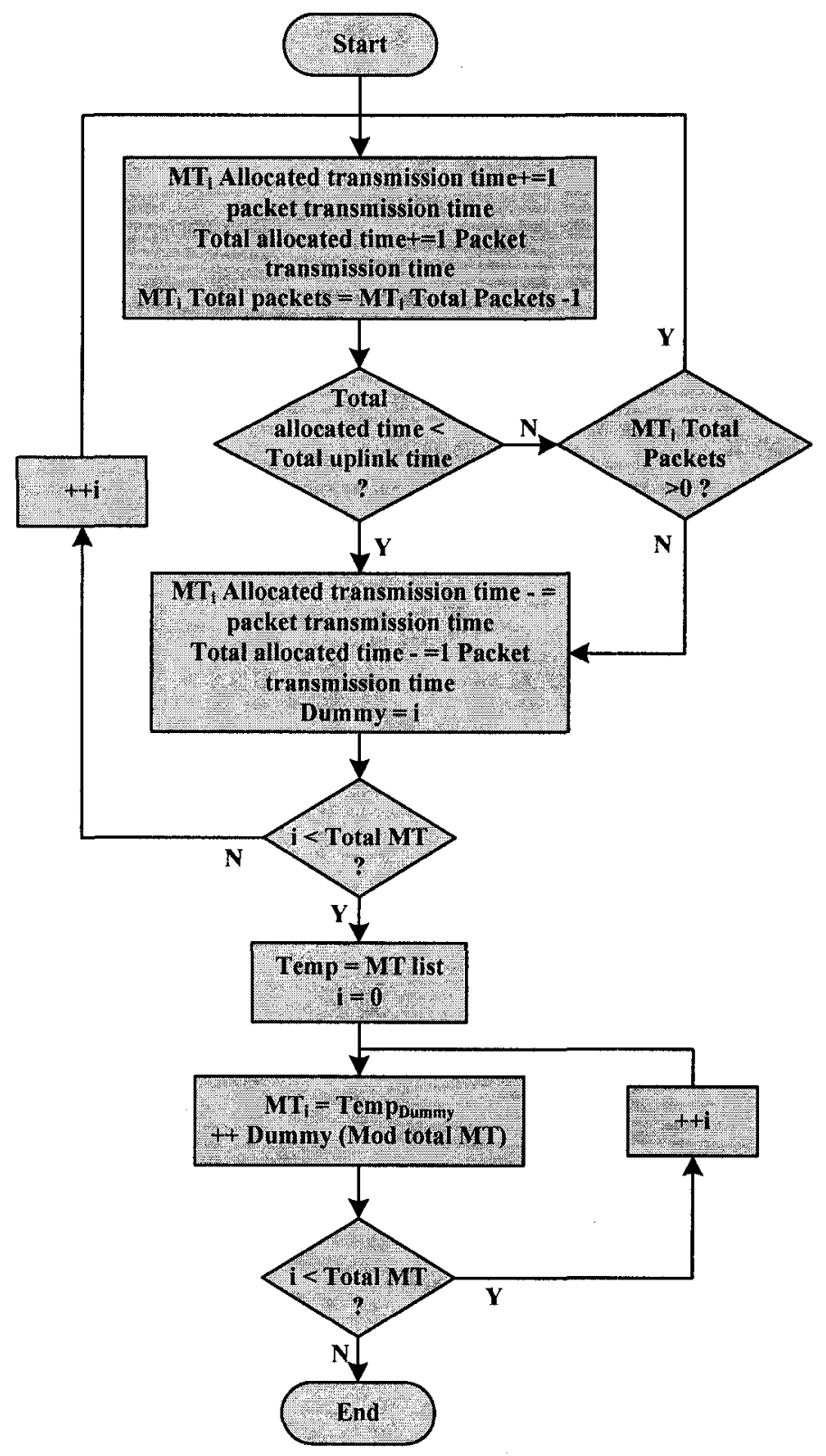

Figure 3.1. Flow chart MORR scheme. 


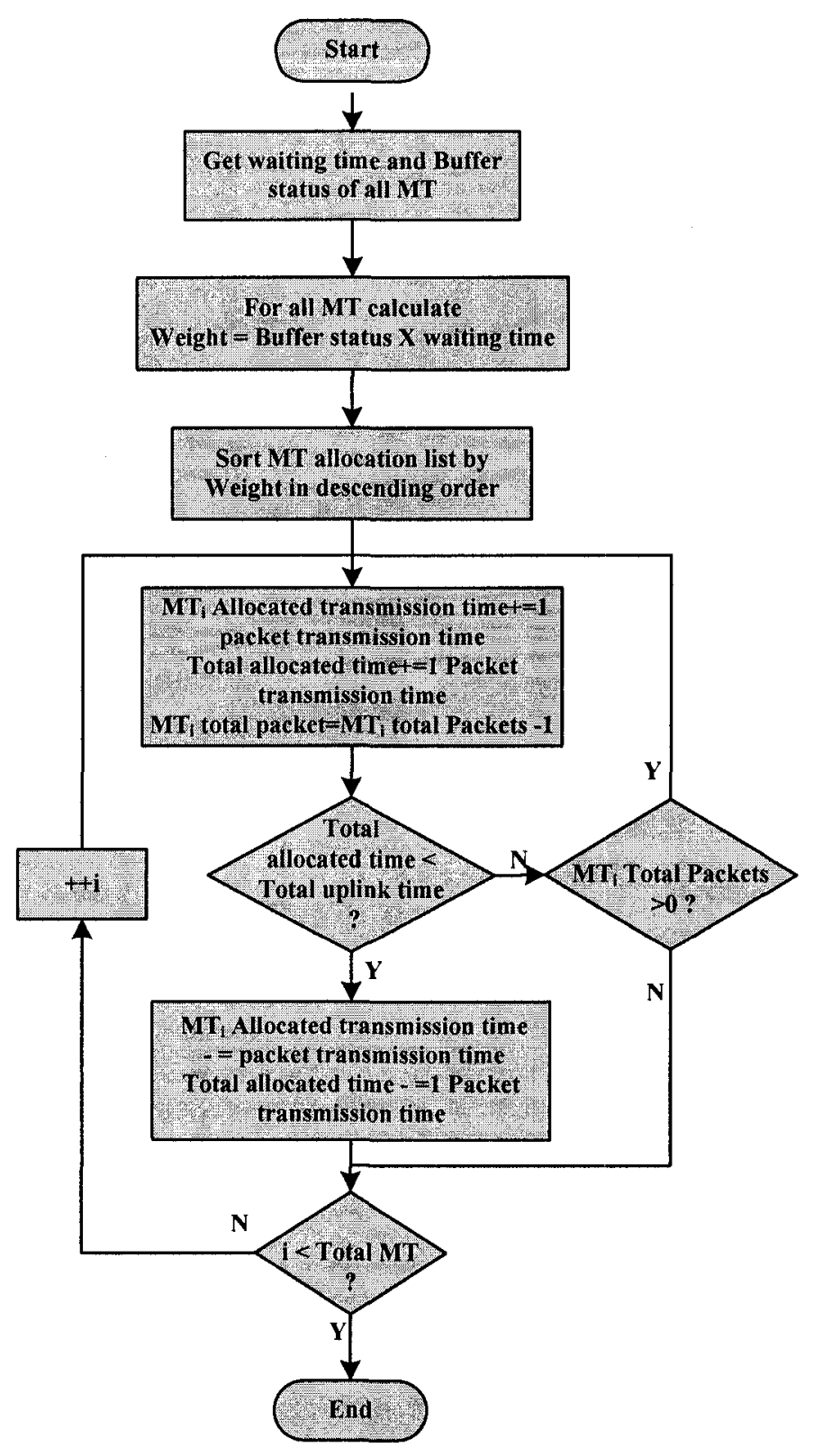

Figure 3.2. Flowchart for WMORR scheme.

\subsection{Weighted Round Robin (WRR)}

With this scheme, resource is allocated to MTs by using a look up table. Whenever a particular MT is visited, transmission time equal to one PDU is allocated to it. A certain MT is visited again if time is left in fame provided that AP has completed its visit to all other MTs. The look up table for resource allocation is prepared using the addresses of the associated 
MTs and their weight. Weight of the MT is defined as the number of PDUs in the buffer of that MT. The look up table is rearranged and updated in every frame using buffer status of the MTs and MT with more weight is placed higher in the look up table. Basically this scheme works as Round Robin algorithm but only difference is that MTs are polled as per their demand. A flowchart for this scheme is shown in Fig. 3.3.

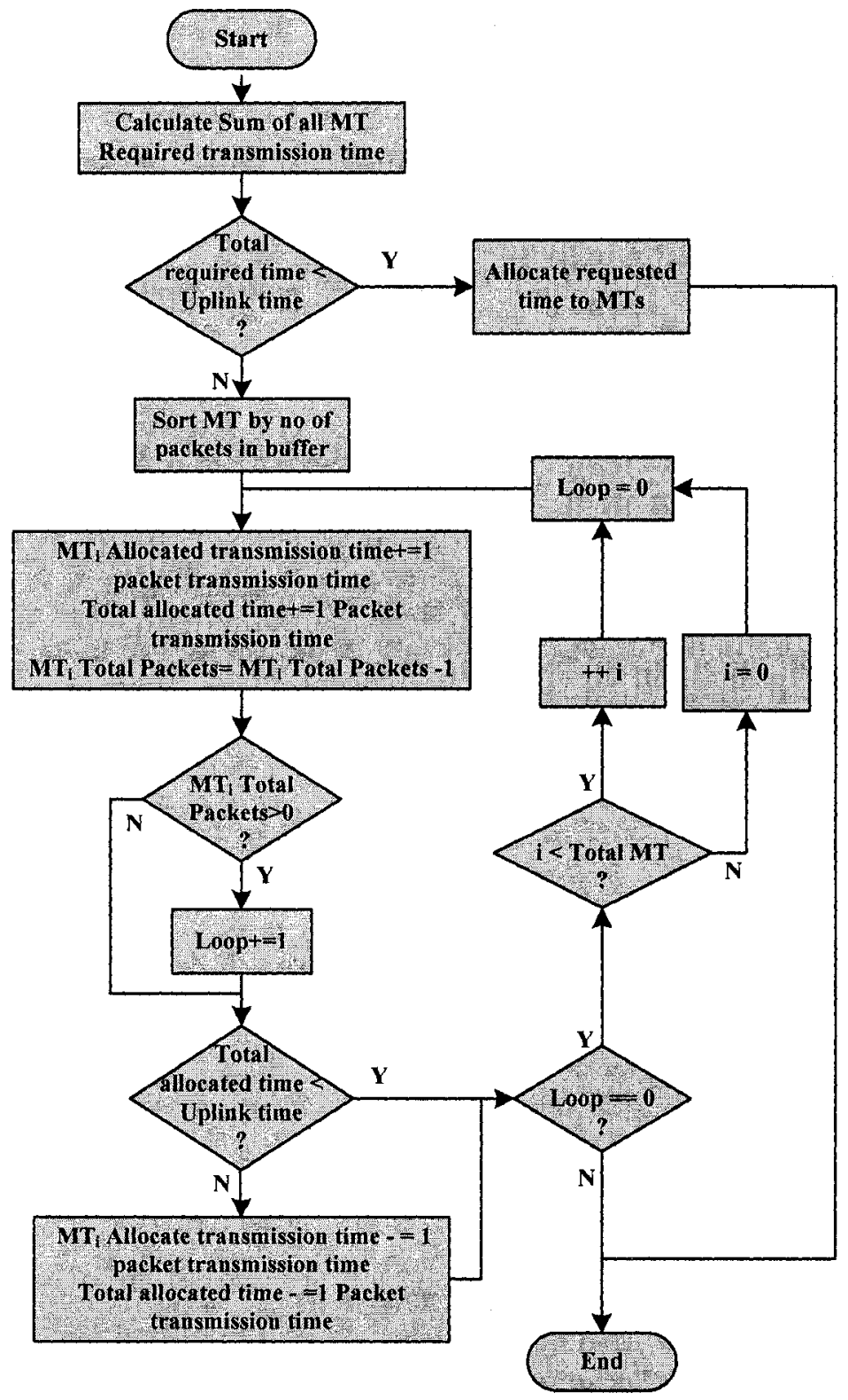

Figure 3.3. Flowchart for WRR scheme. 


\section{Chapter 4:System Setup}

The simulation is done in a module based manner using VC++. Each module is independent and data is transferred from one module to another by predefined global variable in the program. We simulate the $\mathrm{H} / 2$ system for both uplink (Date direction MT to AP) and downlink (Data direction AP to MT) considering best effort packet delivery.

\subsection{Simulation Setup}

In our simulation we choose parameters to operate our system in an open space outdoor environment. We simulate four independent LANs as shown in fig. 4.1. Service area of an AP is the largest distance from the AP where data rate becomes zero. It is determined from the knowledge of physical layer parameters. A central controller (CO) connects all the APs.

All the packets transmitted by MTs have to pass through its associated AP in our network. If a received data packet of by AP has a destination MT which is located in the service area of another AP, packet is routed through the $\mathrm{CC}$ to that $\mathrm{AP}$. For routing, a look up table (LUT) is used where each MT, AP and CC is listed using unique address. We assume that no packet loss occurs in packet exchange process between AP and CC. But we consider the effects of packet losses in the link between MTs and APs. In the simulation, we set a variable in each PDU. When the PDU is transmitted by MT or AP we call $U(0,1)$ and store the outcome in the variable of the PDU. At the destination of the PDU we compare the value 
of this variable with the acceptable error value which is 0.01 (Error rate 1 out of 100 PDUs). If the value of the variable is equal to or greater than 0.01 we took the PDU as successful one.

In our system APs communicate with the CC using HIPERLINK standard [7] at data rate $155 \mathrm{Mbits} / \mathrm{s}$. Queuing delay is added with a PDU when it pass through CC. Queuing delay depends on the number of PDUs sent to $\mathrm{CC}$ by APs. Using the model for $\mathrm{M} / \mathrm{M} /$ 1queuing [21] this average delay is calculated using equation 4.1, where $\mu$ is the PDU service rate of $C C$ and $\lambda$ is the PDU arrival rate to $C C$.

$$
E[T]=\frac{1}{\mu-\lambda} .
$$

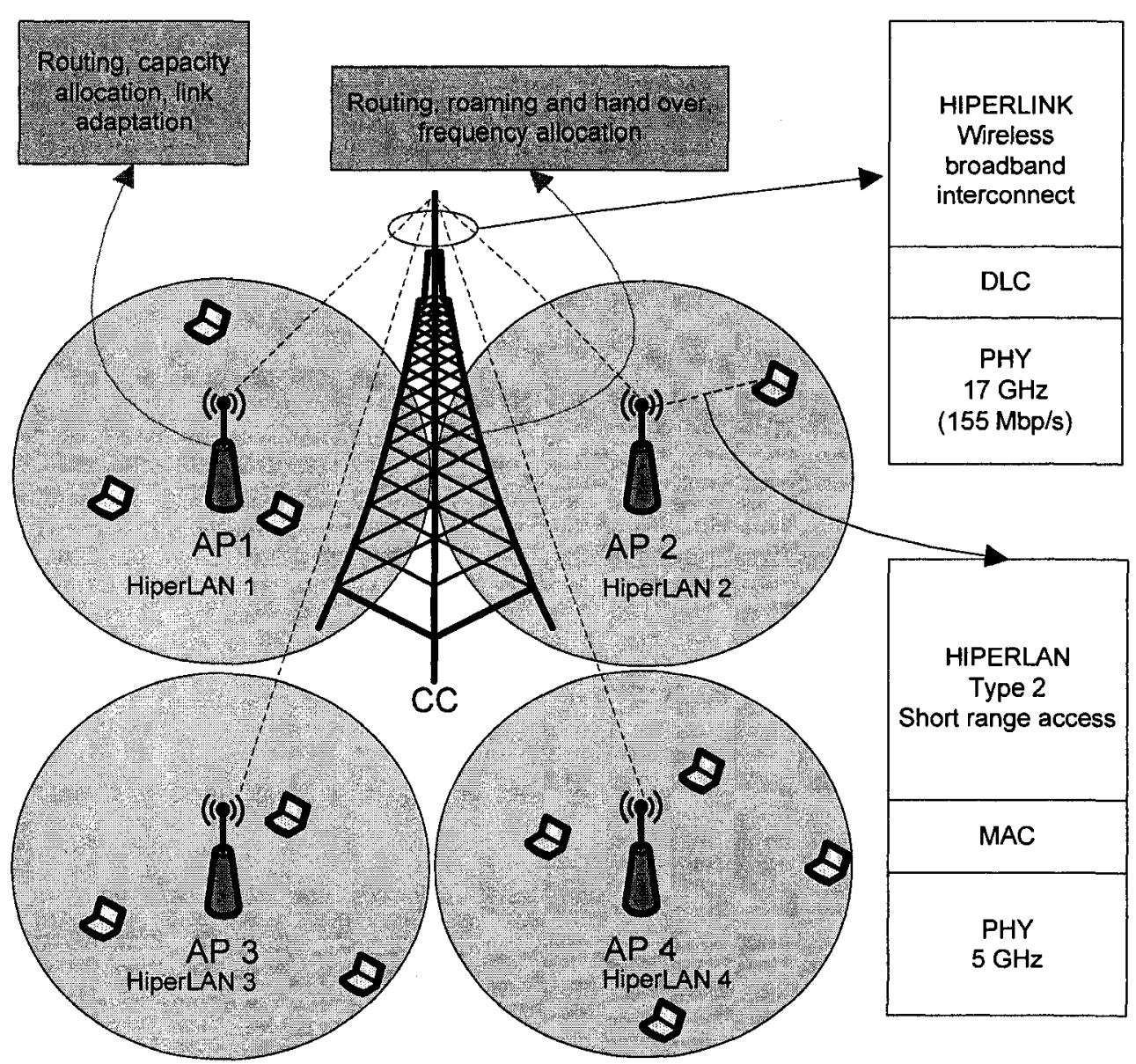

Figure 4.1. Simulation system setup. 


\subsection{Simulation Flowchart}

The length of each MAC frame is $2 \mathrm{~ms}$. The frame is divided into number of short time phases. Data from MAC layer mapped to physical burst using the physical layer transmission rate as shown in table 4.1. We simulate the system in modular manner where each module corresponds to a short time phase of a TDMA/TDD frame. Using the operation parameters of table 4.1 we developed flow charts of each short phase of the TDMA/TDD frame and realized them by the VC++ code. The flowchart for the whole system is shown in fig. 4.2. It contains four sub-routines and each one represents one short time phase. Subroutines for the broadcast phase, uplink phase, downlink phase and random access phase is shown in fig. 4.3, $4.4,4.5$ and 4.6 respectively.

Table 4.1. Operation Parameter Setting

\begin{tabular}{|c|c|c|}
\hline Transport Channel & Modulation \& Code rate & Tx time $(\mu \mathrm{s})$ \\
\hline \multicolumn{3}{|l|}{ Broadcast PDU Train } \\
\hline Preamble & & 16 \\
\hline $\mathrm{BCH}$ & BPSK, code rate $1 / 2$ & 20 \\
\hline $\mathrm{FCH}$ & BPSK, code rate $1 / 2$ & Multiple of 36 \\
\hline$\overline{\mathrm{ACH}}$ & BPSK, code rate $1 / 2$ & 12 \\
\hline \multicolumn{3}{|l|}{ Downlink PDU train } \\
\hline Preamble & & 8 \\
\hline $\mathrm{SCH}$ & BPSK, code rate $1 / 2$ & 12 \\
\hline \multirow[t]{4}{*}{ LCH } & BPSK, code rate $1 / 2,3 / 4$ & 72,48 \\
\hline & QPSK, code rate $1 / 2,3 / 4$ & 36,24 \\
\hline & 16QAM, code rate $9 / 16,3 / 4$ & 16,12 \\
\hline & $64 \mathrm{QAM}$, code rate $3 / 4$ & 8 \\
\hline \multicolumn{3}{|l|}{ Uplink PDU train } \\
\hline Preamble (Short) & & 12 \\
\hline $\mathrm{SCH}$ & \multicolumn{2}{|c|}{ Same as downlink } \\
\hline $\mathrm{LCH}$ & \multicolumn{2}{|c|}{ Same as downlink } \\
\hline Guard time & & 2 \\
\hline \multicolumn{3}{|l|}{ Random access } \\
\hline Preamble (Short) & & 12 \\
\hline $\mathrm{RCH}$ & BPSK, code rate $1 / 2$ & 12 \\
\hline Guard time & & 2 \\
\hline Radio turn-around $(\max )$ & & 6 \\
\hline
\end{tabular}




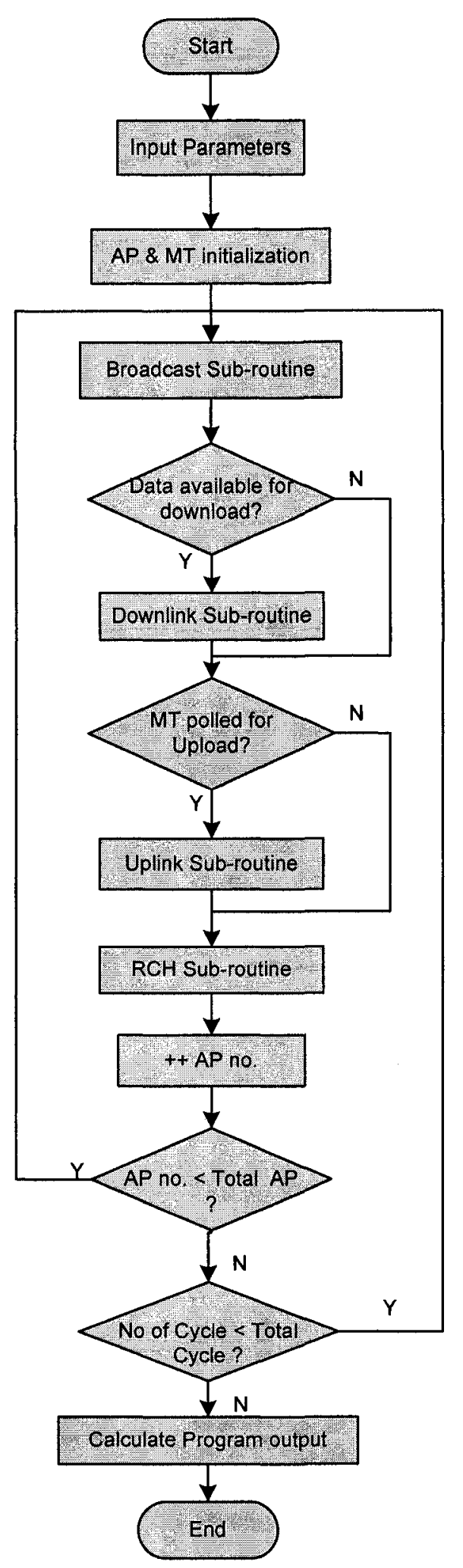

Figure 4.2. Flowchart for the system.

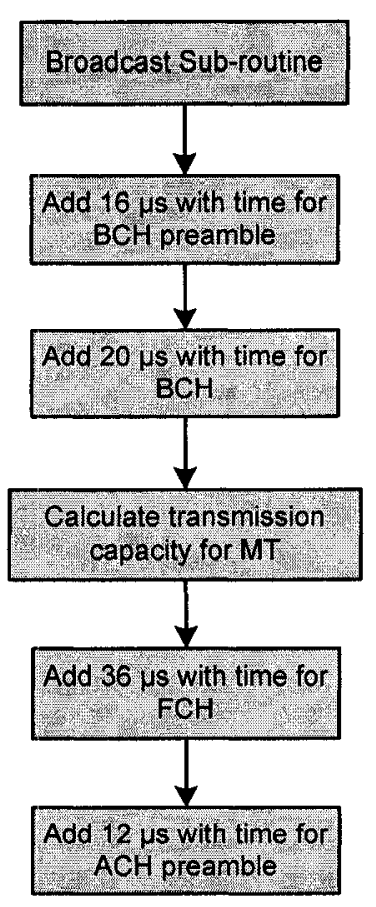

Figure 4.3. Flowchart for Broadcast phase.

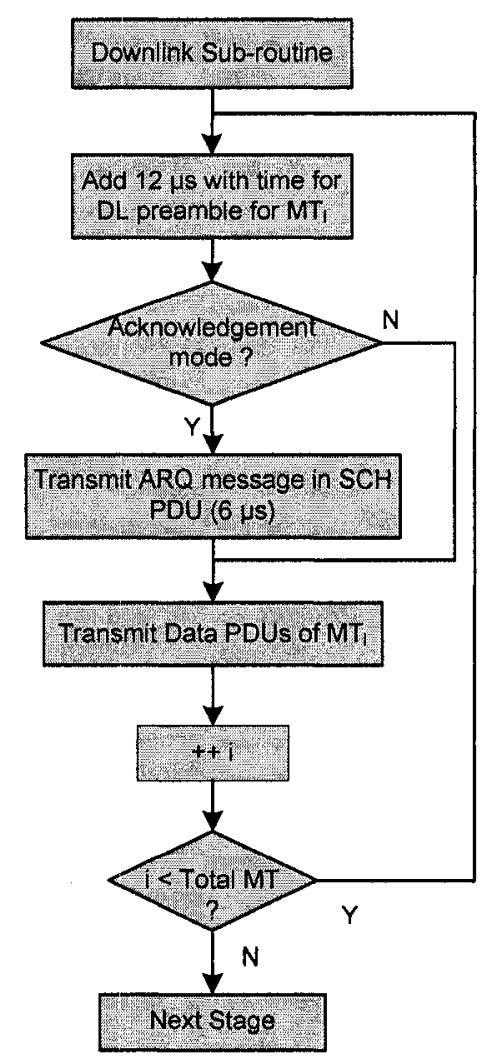

Figure 4.4. Flowchart for Downlink phase. 


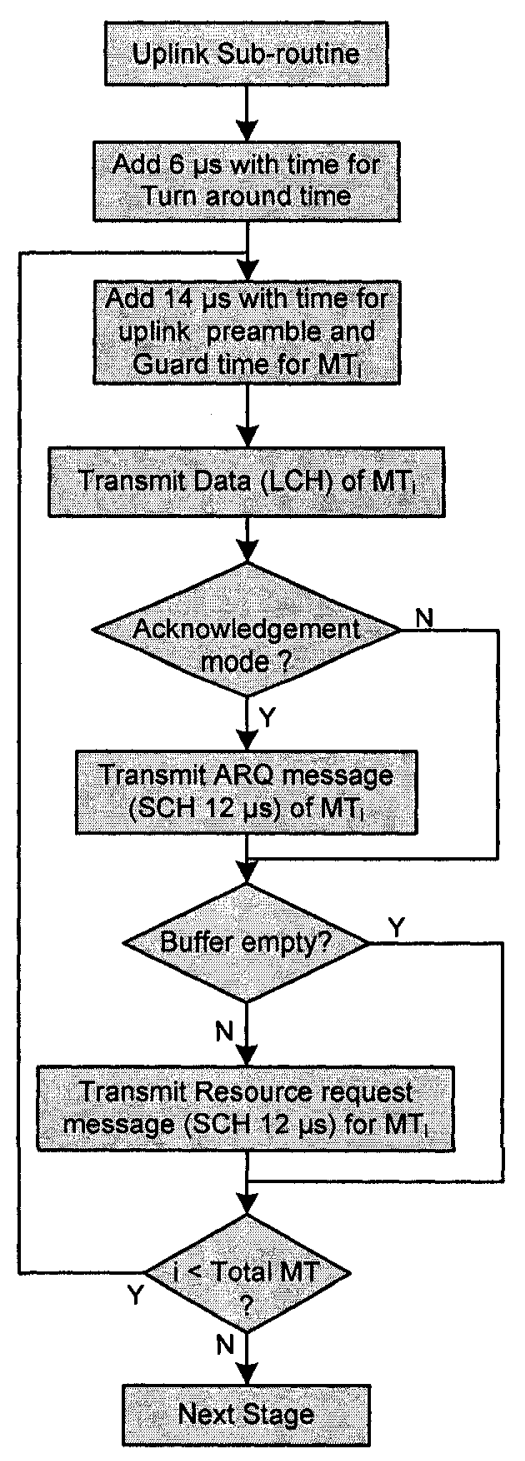

Figure 4.5. Flowchart for Uplink phase.

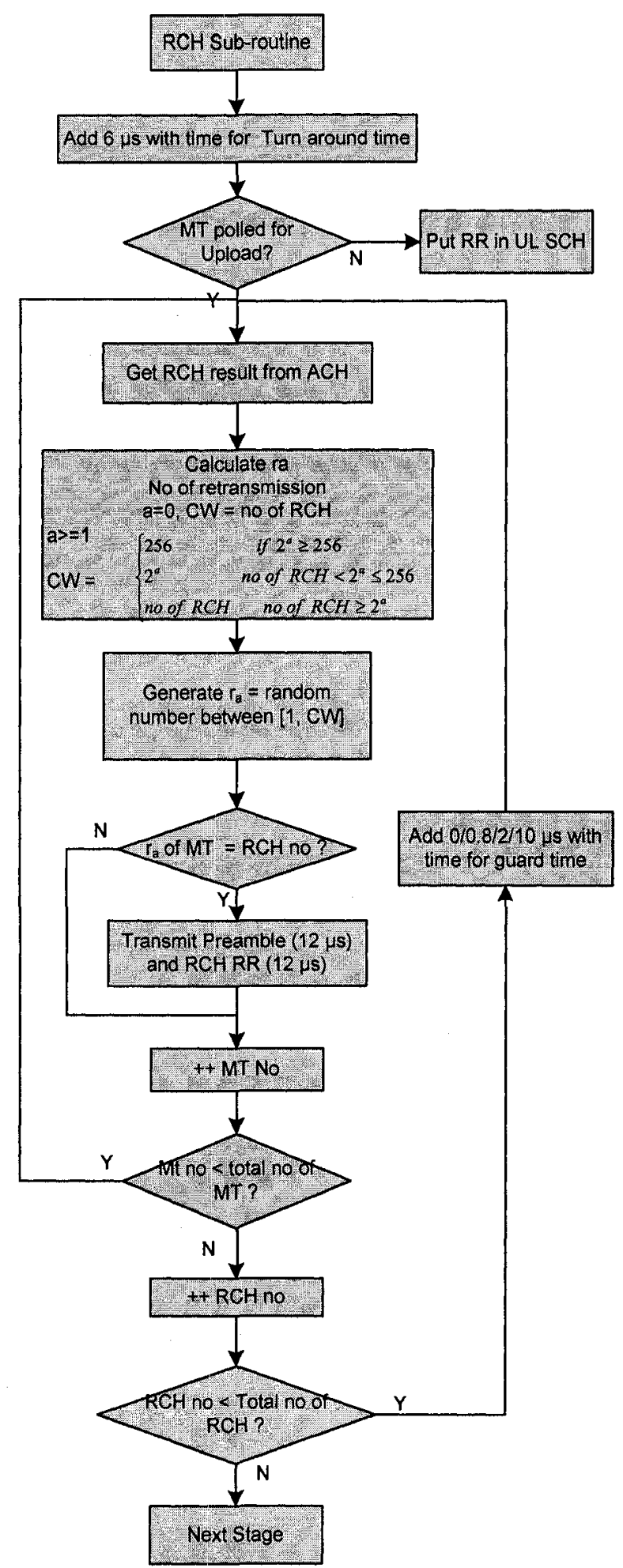

Figure 4.6. Flowchart for $\mathrm{RCH}$ phase. 


\subsection{Data rate Calculation}

We simulate the system in outdoor environment i.e. Channel $\mathrm{E}$ and consider Omnidirectional antenna having height $12 \mathrm{~m}$ and EIRP $1 \mathrm{~W}$. Each AP uses link adaptation procedure to select the appropriate data rate for the associated MTs. For link adaptation at a particular $\mathrm{C} / \mathrm{N}$ value, choice is made in favour of the highest value of achievable data rate considering Modulation and PER (PDU Error Rate) for that value of $\mathrm{C} / \mathrm{N}$. Using the following steps and the simulated PER vs $\mathrm{C} / \mathrm{N}$ graph (Fig. 4.7) [23] we generate link adaptation curve (fig. 4.8) and table 4.2, considering $1 \%$ PER specification.

1. At the receiver (MT or $\mathrm{AP})$ side nose floor $\mathrm{N}$ is calculated by equation 4.2 . Where the $\mathrm{H} / 2$ bandwidth, $\mathrm{B}=20 \mathrm{MHz}$ and Noise factor, $\mathrm{NF}=6 \mathrm{~dB}$.

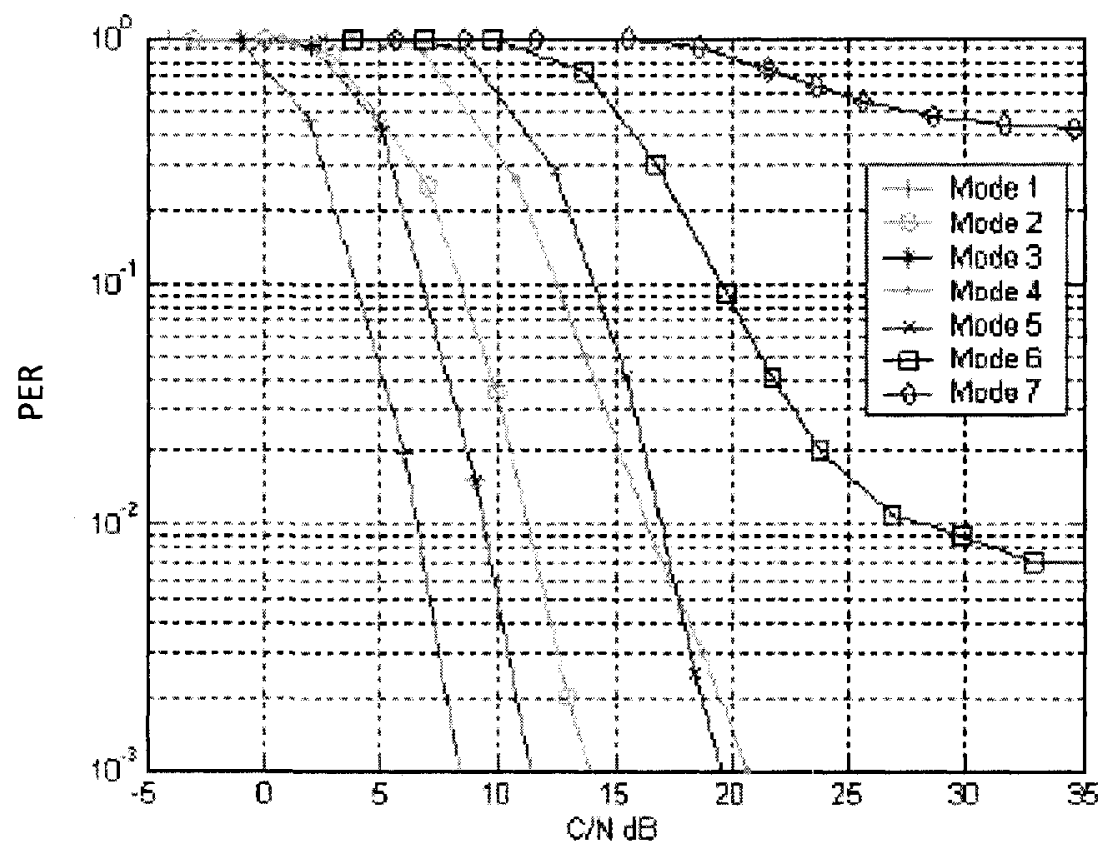

Figure 4.7. PER vs $\mathrm{C} / \mathrm{N}$ curve for channel $\mathrm{E}$. 
2. For a particular mode from the PER vs. $\mathrm{C} / \mathrm{N}$ curve (Fig. 4.7) [23], we determine the highest value of carrier to noise ratio $(C / N)$ for which the specification of 0.01 PER is maintained.

3. We use this value of the $\mathrm{C} / \mathrm{N}$, the value of $\mathrm{N}$ from step 1 and consider $2 \mathrm{~dB}$ as implementation factor $\left(\mathrm{L}_{\text {imp }}\right)$ in equation 4.3 to calculate the minimum received carrier power $\left(C_{R}\right)$.

$$
C_{R}=N+\frac{C}{N}+L_{i m p l}
$$

4. The path loss $\left(P_{L}\right)$ for this $C_{R}$ is calculated by equation 4.4 , where transmitted carrier power $\left(\mathrm{C}_{\mathrm{T}}\right)$ is $30 \mathrm{dBm}$.

$$
P_{L}=C_{T}-C_{R}
$$

5. Using the value of path loss we determine the distance for this path loss using path loss model (equation 4.5) [22], where $b=20$ and $n=4.5$ (For antenna height $=12 \mathrm{~m}$ in urban area and NLOS).

$$
P_{L}=b+10 n \log _{10} d \text {. }
$$

6. This is the highest distance or range of a particular mode, up to where the mode maintains 0.01 PER specification.

7. We followed the steps 2 to 6 for all the modes and determine the range of each mode.

8. Finally, for a particular distance we choose a mode that gives the highest data rate.

In our simulation we used table 4.2 as LUT to determine uplink or downlink data rate for any distance between MT and AP. It is to be noted that for this particular channel Mode 2 and Mode 7 is never used because of the very high PER in this channel. 
Table 4.2. LOOK UP TABLE FOR MODE SELECTION.

\begin{tabular}{c|c|c}
\hline Distance (m) & $\mathbf{C} / \mathbf{N}$ (dB) & Mode \\
\hline $137.26-119.55$ & $6.8-9.5$ & 1 \\
\hline $119.55-83.56$ & $9.5-16.5$ & 3 \\
\hline $83.56-80.41$ & $16.5-17.25$ & 4 \\
\hline $80.41-47.59$ & $17.25-27.5$ & 5 \\
\hline$<47.59$ & $27.5+$ & 6 \\
\hline
\end{tabular}

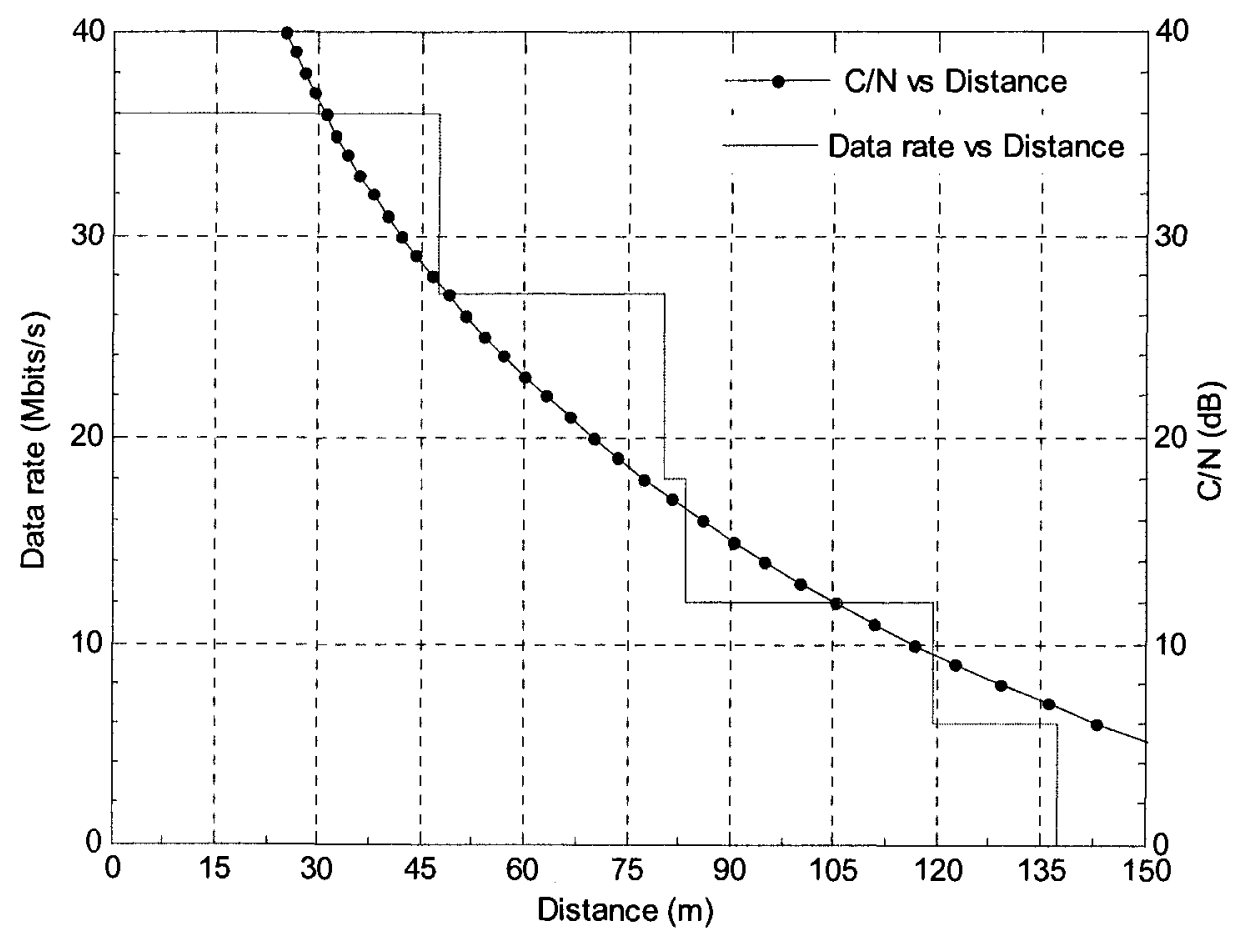

Figure 4.8. C/N Vs Distance curve for outdoor channel.

\subsection{MT Position and Mobility}

In our simulation MTs are scattered randomly inside the service area of an AP. Position of a mobile terminal is randomized using uniform distribution. In our simulation we simulate the condition where MTs are free to move within the service area of AP but cannot leave their respective AP. In our simulation we let the MTs to move at a maximum rate of $10 \mathrm{~m} / \mathrm{s}$. When a MT overtake the boundary of the service area of its associated $\mathrm{AP}$ we turned it back inside the service area of $\mathrm{AP}$ and assign a position at the edge of the boundary of the service area. A 
snapshot of transmission rate of a typical MT during simulation runtime is presented in fig. 4.8 .

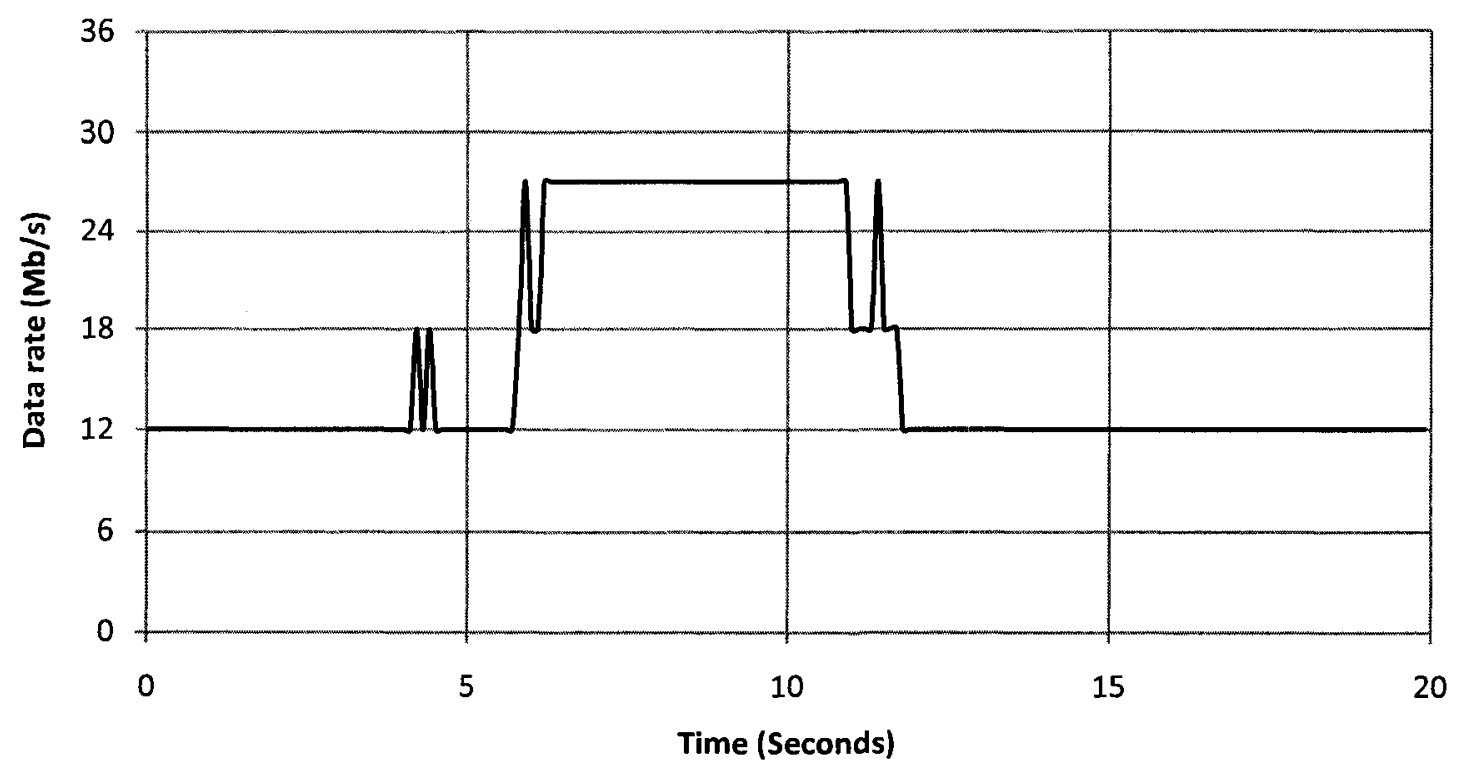

Figure 4.9. A snapshot of transmission rate of a typical MT during simulation.

To make an equal condition for performance measurement of the allocation policies we stored the location of each MT in the WLAN area after each TDMA/TDD frame in a separate data file at the beginning of our simulation. This position indicator data file is used as input for MT position in the rest of the simulation program.

\subsection{Simulation Criteria}

\section{- Average Number of PDUs in Buffer and its Variance}

PDUs received from the DLC layer are stored in the buffer of each MT for transmission in the TDMA frame. As length of each TDMA frame is fixed, buffers in the MTs grow up if discharge rate is lower than the arrival rate. Average number of PDUs in the buffer of MTs indicates the buffer occupancy of the MTs. To calculate average number of PDUs in the Buffer, the number of PDUs in the buffer of MTs for the simulation period is averaged by 
equation 4.6 first. This result is averaged again by adding up and dividing by the total number of MTs (equation 4.7), to get Average Number of PDUs in Buffer finally.

$$
\begin{gathered}
B_{i}=\frac{\sum_{j=1}^{\text {Total no.of itaration }} B_{i j}}{\text { Total no.of itaration }} \\
\bar{B}=\frac{\sum_{i=1}^{\text {Total no.of } M T} B_{i}}{\text { Total no.of } M T}
\end{gathered}
$$

Where,

$B_{i j}=$ Number of PDUs in the buffer of $i^{\text {th }}$ MT at $j^{\text {th }}$ itration.

$B_{i}=$ Average number of PDUs in the buffer of the $i^{\text {th }} M^{T} T$ for the simulation runtime.

$\bar{B}=$ Average number of PDUs in the Buffer.

The variance of the average number of PDUs in the buffer of MTs is calculated by the equation 4.8 as below:

$$
\sigma_{B}^{2}=\frac{\sum_{i=1}^{T \text { otal no.of } M T}\left(B_{i}-\bar{B}\right)^{2}}{\text { Total no.of } M T-1}
$$

\section{- Probability of Buffer Overflow and its Variance}

PDUs from the DLC connections are stored in the buffer of MT using First In First Out algorithm (FIFO). If the PDU arrival rate is higher than the discharge rate PDUs accumulates in the buffer of MTs. As the buffer size is finite overflow occurs when buffer gets full and any new PDU from the DLC connection is discarded. To calculate the probability of buffer overflow we set a counter in the buffer of the MT. This counter is incremented by 1 , if one PDU is discarded. Average number of buffer overflow for a MT for the simulation runtime is calculated by equation 4.9. This value is averaged over the number of MT to get the Average Probability of Buffer Overflow (equation 4.10). 


$$
\begin{gathered}
O_{i}=\frac{100 \times \sum_{j=1}^{\text {Total no.of itaration }} o_{i j}}{\text { Total no.of itaration }} \\
\bar{O}=\frac{\sum_{i=1}^{\text {Total no.of } M T} o_{i}}{\text { Total no.of } M T}
\end{gathered}
$$

Where,

$\mathrm{O}_{i j}=$ Number of overflow in the buffer of $i^{\text {th }} \mathrm{MT}$ at $\mathrm{j}^{\text {th }}$ itration.

$\mathrm{O}_{\mathrm{i}}=$ Average number of buffer overflow of the $i^{\text {th }} \mathrm{MT}$ for the simulation runtime.

$\bar{O}=$ Probability of Buffer Overflow.

The variance of the buffer overflow of MTs is calculated by the equation 4.11 as below:

$$
\sigma_{O}^{2}=\frac{\sum_{i=1}^{\text {Total no.of } M T}\left(O_{i}-\bar{O}\right)^{2}}{\text { Total no.of } M T-1}
$$

\section{- Average PDU delivery time and its variance}

PDU delivery time is the total amount of time required by a PDU to reach its destination after generation. In the system it can happen in two ways:

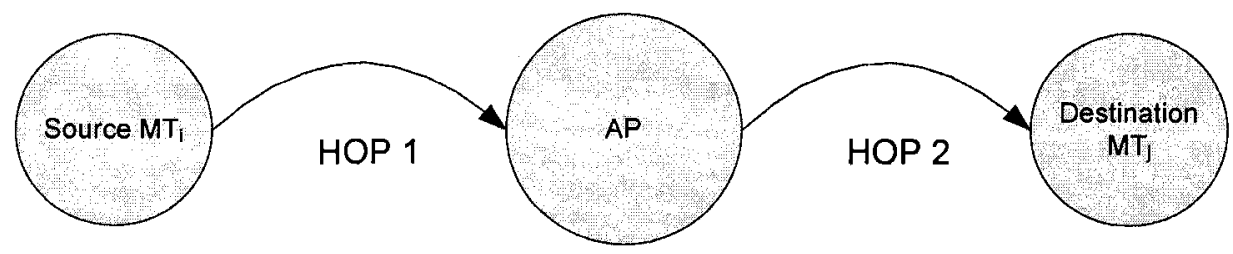

Figure 4.10. PDU delivery event under in same AP.

- If the source and the destination MT of a PDU is located inside the service area of an $\mathrm{AP}$ (Fig. 4.1), total time is the summation of time elapsed by the PDU in the buffer of source MT, transmission time by source MT, time elapsed by the PDU in the buffer of $\mathrm{AP}$ and transmission time of the PDU by AP (Fig. 4.10). 


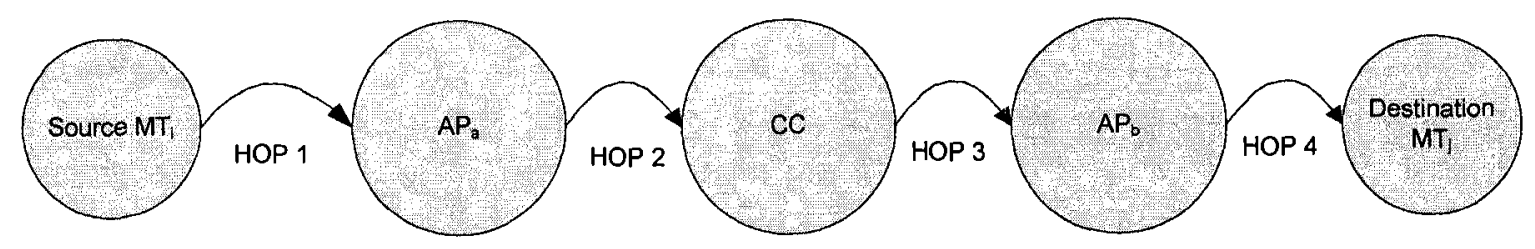

Figure 4.11. PDU delivery event under in different AP.

- If destination MT of the PDU is located in the service area of AP other than the AP of the source MT, total time is the summation of time elapsed by the PDU in the buffer of source MT, transmission time by source MT, time elapsed by the PDU in the buffer of $A P$ and $C C$, queuing delay in the buffer of $C C$ and transmission time of the PDU by $\mathrm{AP}$ and $\mathrm{CC}$ (Fig. 4.10).

To calculate the average PDU delivery time, average delivery time for PDUs of all the MTs is calculated first by equation 4.12 . These results are added and divided by the total number of MTs to get the final result (equation 4.13).

$$
\begin{aligned}
& D T_{i}=\frac{\sum_{j=1}^{T o t a l} \text { no.of Successful PDU } D T_{i j}}{\text { Total no.of Successful PDUs of } M T_{i}} \\
& \overline{D T}=\frac{\sum_{i=1}^{\text {Total no.of } M T} D T_{i}}{\text { Total no.of } M T}
\end{aligned}
$$

Where,

$\mathrm{DT}_{\mathrm{ij}}=$ Delivery time for $\mathrm{j}^{\text {th }} \mathrm{PDU}$ of $\mathrm{i}^{\text {th }} \mathrm{MT}$.

$\mathrm{DT}_{\mathrm{i}}=$ Average PDU delivery time for the $\mathrm{i}^{\text {th }} \mathrm{MT}$.

$\overline{D T}=$ Average PDU delivery time.

The variance of the PDU delivery time is calculated by the equation 4.14 as below:

$$
\sigma_{D T}^{2}=\frac{\Sigma_{i=1}^{\text {Total no.of } M T}\left(D T_{i}-\overline{D T}\right)^{2}}{\text { Total no.of } M T-1}
$$




\section{- Average Overhead in uplink phase and its variance}

We added overhead in the uplink phase of TDMA/TDD frame of all user and expressed it as percentage of total uplink time of an AP. This result is averaged over the total number of frames to determine the overhead in the uplink phase of the AP as in equation 4.15. We calculate the average overhead in uplink phase by adding up the overhead in uplink phase of each AP and dividing the summation by total number of APs as in equation 4.16 .

$$
\begin{gathered}
\eta_{U L_{i}}=\frac{\sum_{j=1}^{\text {Total no.of Frames } \frac{U T o_{i j} \times 100}{U T_{i j}}}}{\text { Total no.of Frames }} \\
\overline{\eta_{U L}}=\frac{\sum_{i=1}^{\text {Total no.of } A P} \eta_{U L_{i}}}{\text { Total no.of } A P}
\end{gathered}
$$

Where,

$U T_{i j} \quad=$ Total uplink time of $j^{\text {th }}$ TDMA frame of $\mathrm{i}^{\text {th }}$ AP.

$U T O_{i j}=$ Summation of all user overhead in $j^{\text {th }}$ frame of $i^{\text {th }}$ AP.

$\eta_{U L_{i}} \quad=$ Average uplink overhead for the $\mathrm{i}^{\text {ith }} \mathrm{AP}$.

$\overline{\eta_{U L}} \quad=$ Average uplink overhead.

The variance of the uplink overhead percentage is calculated by equation 4.17:

$$
\sigma_{\eta U L}^{2}=\frac{\sum_{i=1}^{\text {Total no.of } A P}\left(\eta_{U L_{i}}-\overline{\eta_{U L}}\right)^{2}}{\text { Total no.of AP-1 }}
$$

\section{- Average Overhead in Downlink phase and its variance}

We added overhead in the downlink phase of TDMA/TDD frame of all user and expressed it as percentage of total downlink time of an AP. This result is averaged over the 
total number of frames to determine the overhead in the downlink phase of the AP as in equation 4.18. We calculate the average overhead in downlink phase by adding up the overhead in downlink phase of each AP and dividing the summation by total number of APs as in equation 4.19.

$$
\begin{gathered}
\eta_{D L_{i}}=\frac{\sum_{j=1}^{\text {Total no.of Frames } \frac{D T O_{i j} \times 100}{D T_{i j}}}}{\text { Total no.of Frames }} \\
\overline{\eta_{D L}}=\frac{\sum_{i=1}^{\text {Total no.of } A P} \eta_{D L_{i}}}{\text { Total no.of } A P}
\end{gathered}
$$

Where,

$D T_{i j}=$ Total downlink time of $j^{\text {th }}$ TDMA frame of $i^{\text {th }}$ AP.

$D T O_{i j}=$ Summation of all user overhead in $j^{\text {th }}$ frame of $i^{\text {th }} \mathrm{AP}$.

$\eta_{D L_{i}}=$ Average downlink overhead percentage for the $i^{\text {th }}$ AP.

$\overline{\eta_{D L}} \quad=$ Average downlink overhead.

The variance of the downlink overhead percentage is calculated by the equation 4.20 as below:

$$
\sigma_{\eta D L}^{2}=\frac{\sum_{i=1}^{\text {Total no.of } A P}\left(\eta_{i}-\overline{\eta_{D L}}\right)^{2}}{\text { Total no.of } A P-1}
$$




\section{Chapter 5:Simulation results}

We run a large number of iterations, equivalent to 20 seconds in real time for the simulation setup of chapter 4 , where each iteration represents one TDMA/TDD frame. To measure the performance of the resource allocation schemes, we have simulated our system for two cases:

- Fixed amount of data generation per TDMA/TDD frame to represent idle state of web browsing.

- Random amount of data generation per TDMA/TDD frame to represent busy state of web browsing.

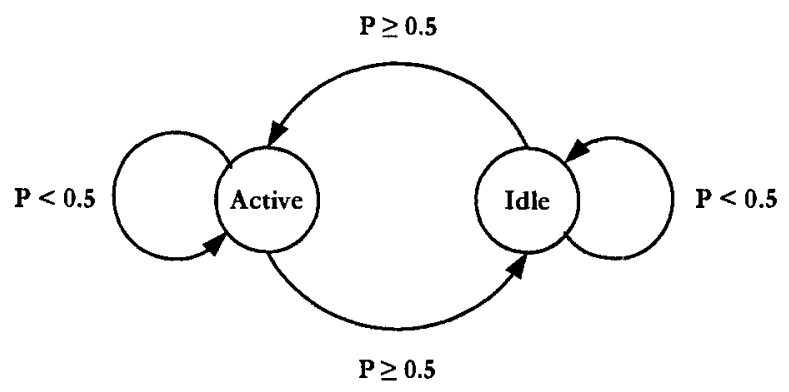

Figure 5.1. ON-OFF model.

\subsection{Fixed Burst}

In the simulation we generate a fixed amount of data (1 PDU or 54 bytes) per time period of $2 \mathrm{~ms}$ using the ON-OFF model as shown in fig. 5.1. We consider one TDMA frame or $2 \mathrm{~ms}$ as length of $\mathrm{ON}$ or OFF period for PDU arrival event. In our ON-OFF model, the data 
generator changes its state from $\mathrm{ON}$ to $\mathrm{OFF}$ or from $\mathrm{OFF}$ to $\mathrm{ON}$ with a probability greater than or equal to 0.5 . We call a $U(0,1)$, if the outcome is greater than or equal to 0.5 the user is ON state. This data generation technique gives out an average data arrival rate to the buffer of each MT equal to $108 \mathrm{~kb} / \mathrm{s}$.

We have calculated the system load by dividing the total requested time in the uplink phase of all MT associated with an $\mathrm{AP}$ by the capacity of that $\mathrm{AP}$ as in equation 5.1. Here the capacity of the AP is the Uplink time in a TDMA frame. Fig. 5.2 shows the load distribution over the number of mobile terminals that we get as outcome of the simulation. The other parameter used to setup the system is summarized in table 5.1.

$$
\begin{aligned}
\text { Load } & =\frac{\text { Total requested time per TDMA frame }}{\text { Total available time in a TDMA frame }} \\
& =\frac{\text { Total } M T \text { per AP } \times \frac{0.5 \times 432}{\text { Average transmission rate per } M T}}{940 \times 10^{-6}}
\end{aligned}
$$

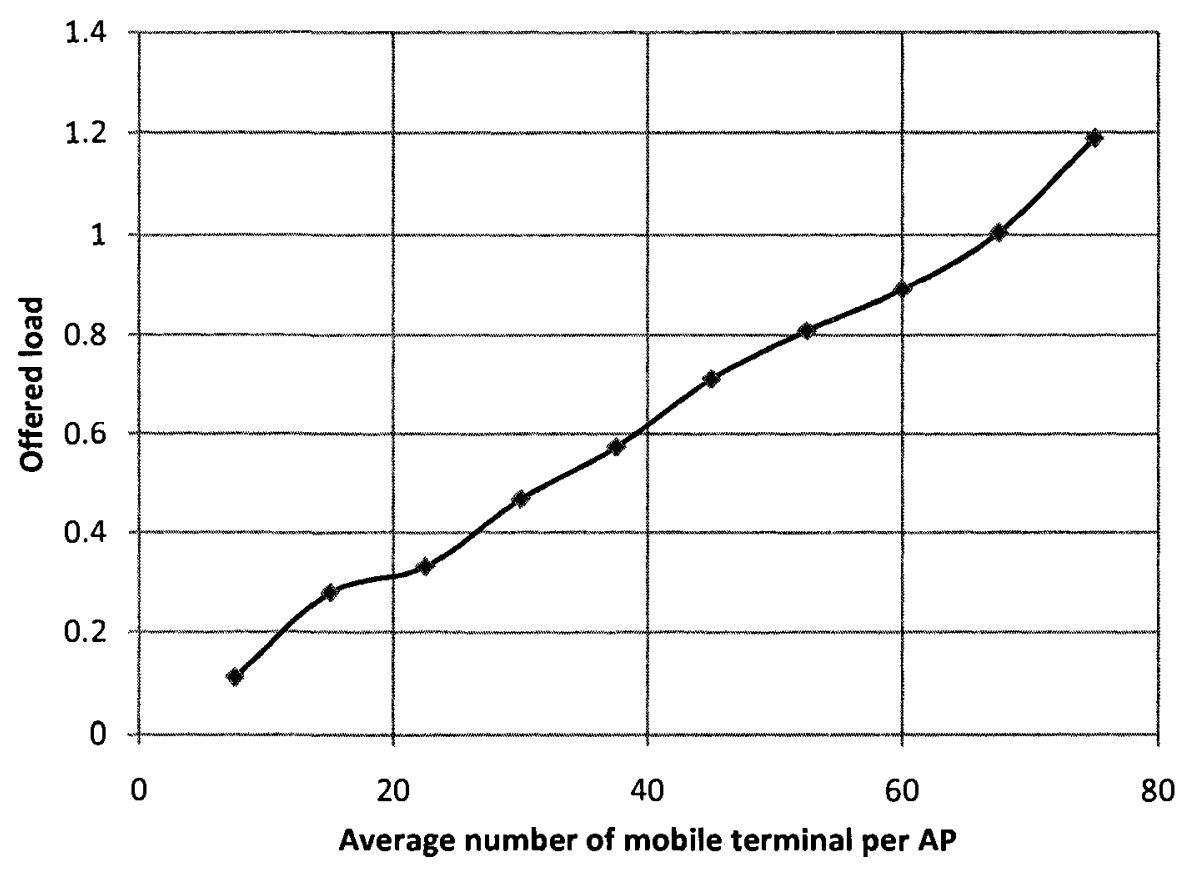

Figure 5.2. Load distribution over number of mobile terminals. 
Table 5.1. Simulation Parameters for Fixed BURST.

\begin{tabular}{l|l}
\hline \multicolumn{2}{c}{ Network Parameters } \\
\hline No of CC & 1 \\
\hline No of AP & 4 \\
\hline Network area & $538 \mathrm{~m} \times 538 \mathrm{~m}$ \\
\hline AP coverage radius & $137.26 \mathrm{~m}$ \\
\hline AP Tx power & $1 \mathrm{Watt}$ \\
\hline Antenna height & $12 \mathrm{~m}$ \\
\hline Antennal type & Omnidirectional \\
\hline AP location & Urban area \\
\hline AP buffer size & 100 PDU \\
\hline CC buffer size & 400 PDU \\
\hline No. of RCH phase & 1 \\
\hline Simulation runtime & 10000 frames \\
\hline DL/UL time & $940 \times 10^{-6} \mathrm{~s}$ \\
\hline \multicolumn{2}{c}{ Source model } \\
\hline LCH PDU length & 54 byte \\
\hline SCH PDU length & 9 byte \\
\hline Max. MT movement rate & $10 \mathrm{~m} / \mathrm{s}$ \\
\hline MT Buffer size & $5 / 10 / 15$ PDU \\
\hline MT Buffer Management & FIFO \\
\hline \multicolumn{2}{l}{} \\
\hline
\end{tabular}

\section{- Average Number of PDUs in Buffer and its Variance}

Fig. 5.3-5.5 shows the average number of PDUs in buffer of MTs as a function of offered load for the three resource allocation policies, where buffer size is 5, 10 and 15 PDUs respectively. In the figures, the WMORR and MORR policies show almost equal performance in terms of buffer occupancy. It is important to note that WMORR and MORR allocation schemes outperform the WRR scheme in terms of buffer occupancy. We also notice that when offered load is around 0.3 there is a sharp change in average number of PDUs in the user buffer. It indicates that before this load total PDU arrived to the MTs is equal to the PDU service rate of MTs. After this load PDU arrival rate becomes higher than the PDU service rate but the system remains in stable as the undelivered PDUs are stored in the buffer for future transmission. 
Buffer size: 5 PDU, Data arrival rate: $108 \mathrm{~kb} / \mathrm{s}$

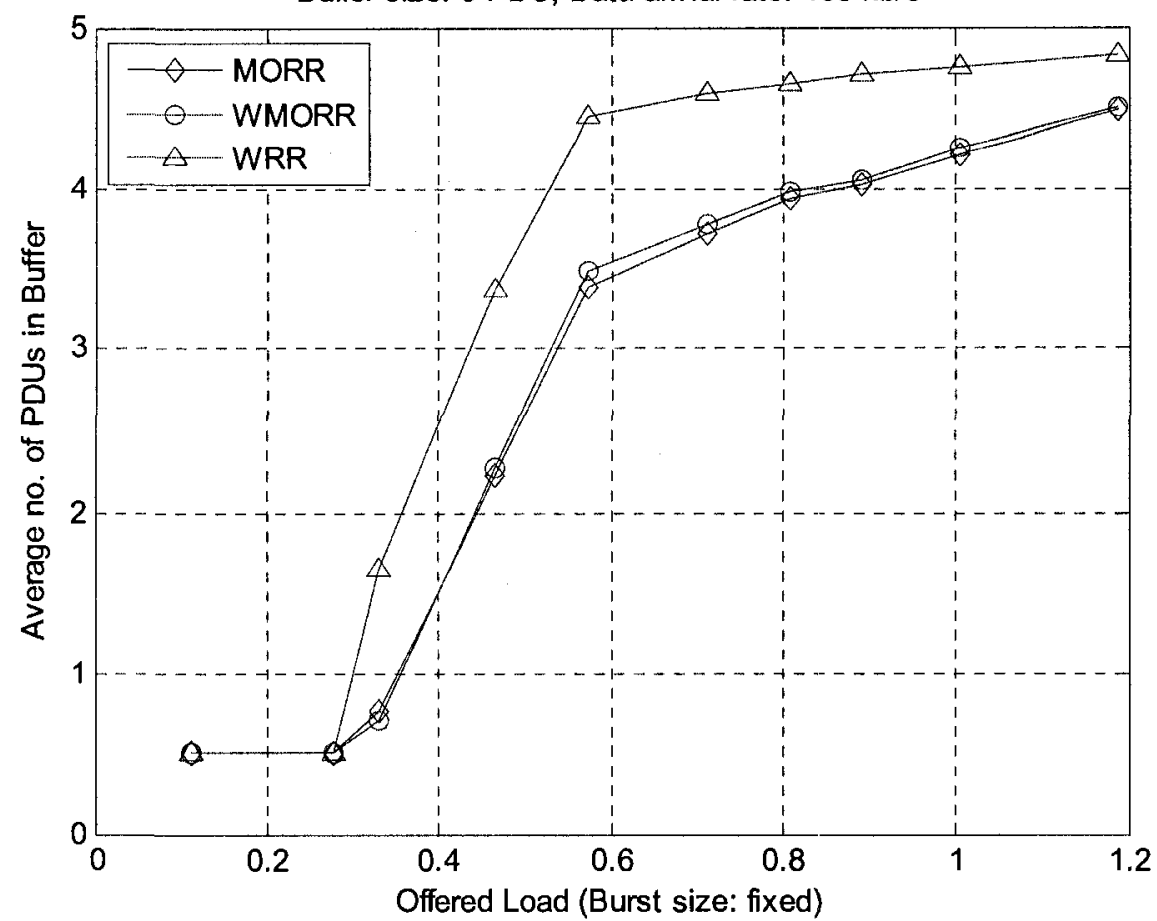

Figure 5.3. Average number of PDUs in Buffer vs Offered load for buffer size 5 PDU at fixed burst.

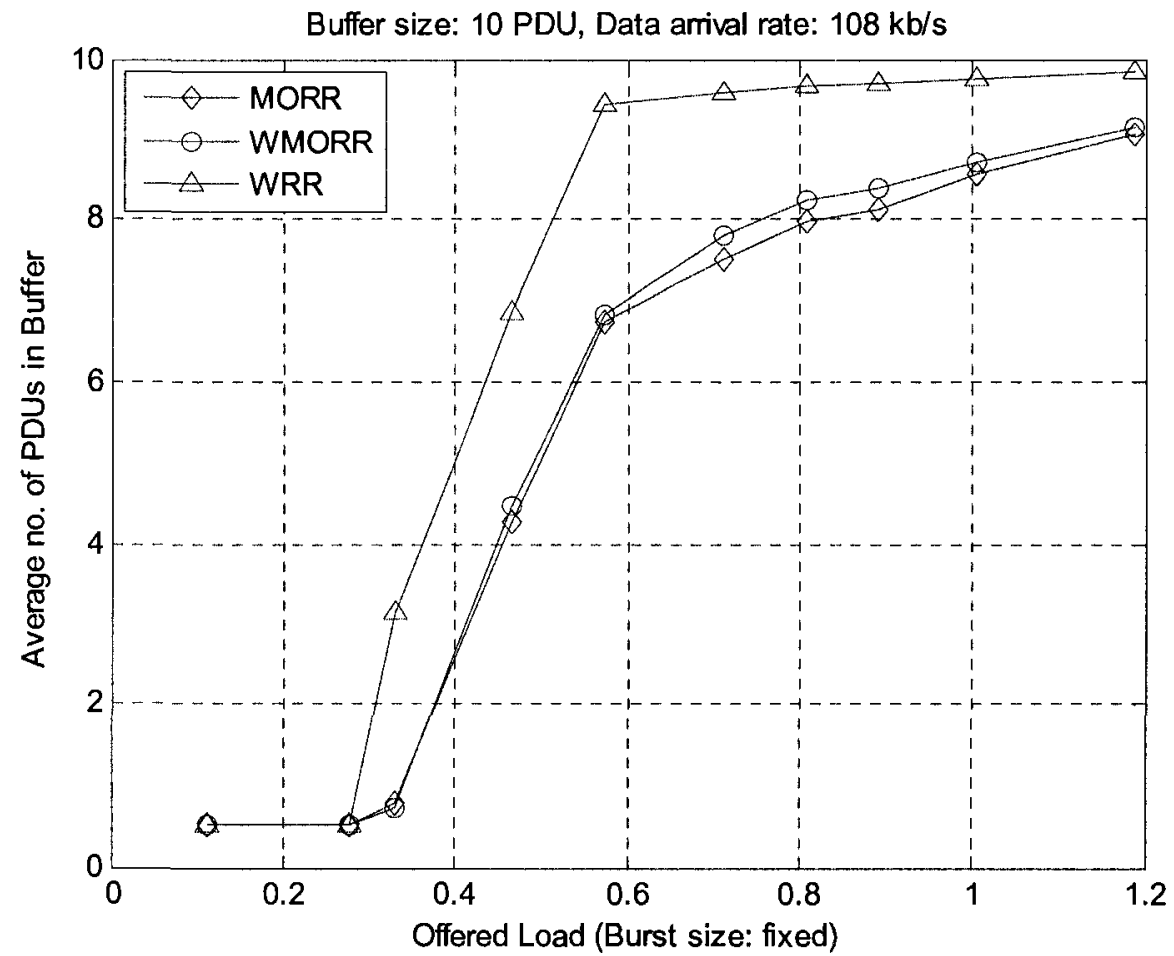

Figure 5.4. Average number of PDUs in Buffer vs Offered load for buffer size $10 \mathrm{PDU}$ at fixed burst. 


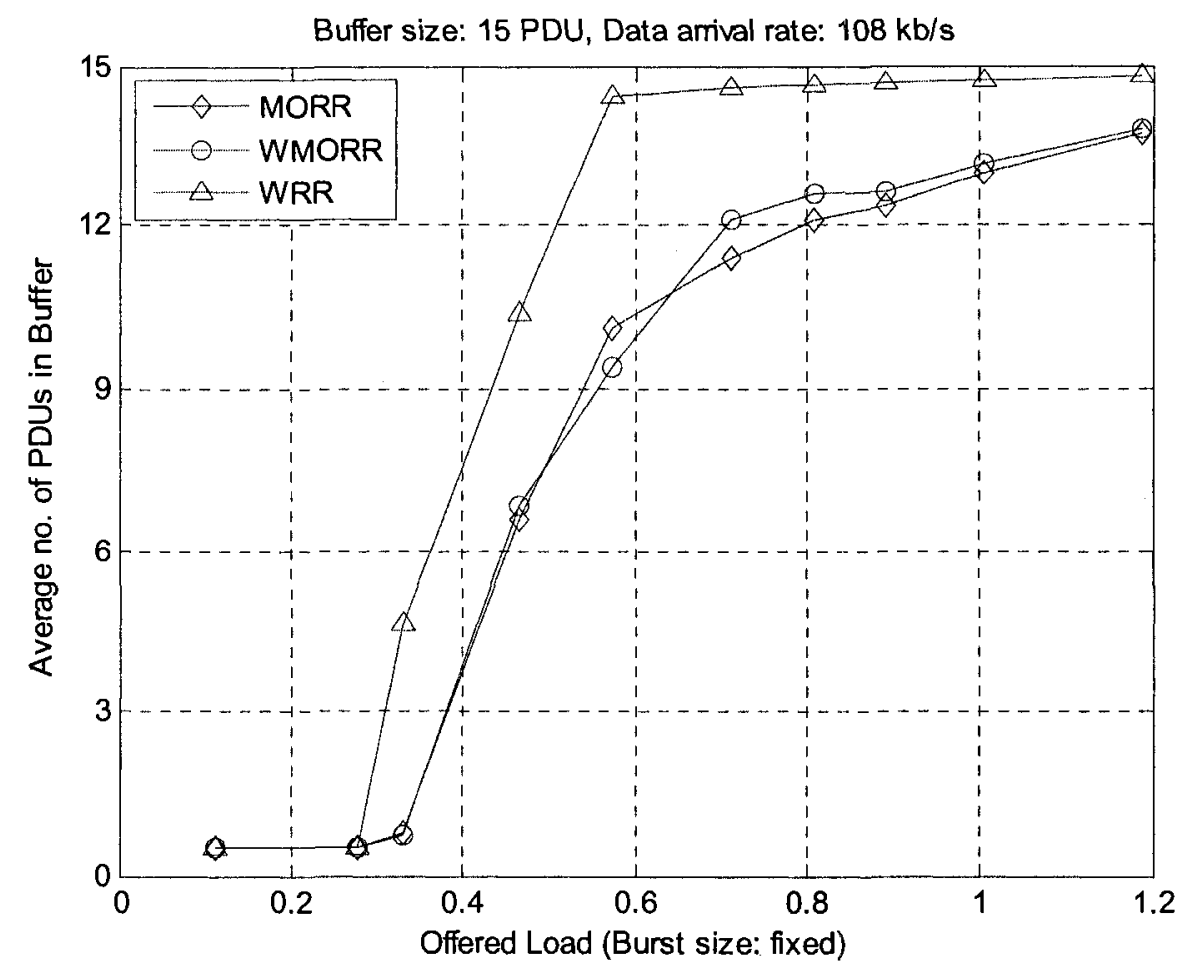

Figure 5.5. Average number of PDUs in Buffer vs Offered load for buffer size 15 PDU at fixed burst.

Fig. 5.6, 5.7 and 5.8 shows the average number of PDUs in buffer of MTs as a function of offered load for the buffer size 5, 10 and 15 PDUs and the figures are for the MORR, WMORR and WRR resource allocation schemes respectively. The figure shows that at very low offered load buffer occupancy is the same for all buffer sizes and as offered load increasess, the number of PDUs in the buffer also increases. Average number of PDUs in the buffer of MTs becomes almost constant at load higher than 0.5 and it gradually becomes equal to the size of the buffer. We also notice that the smaller sized buffer becomes full at lower offered load but for larger sized buffer it happens at very high offered load. 


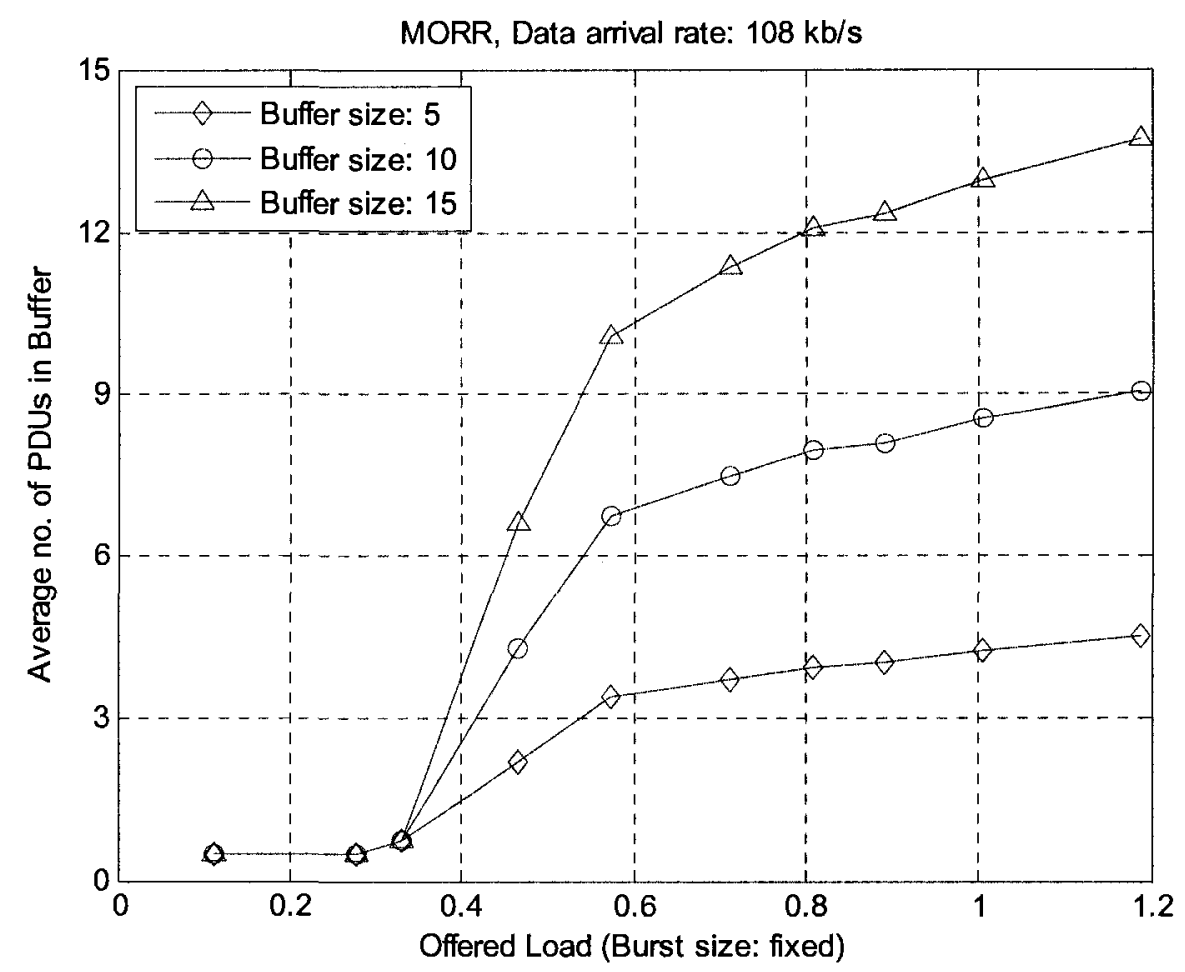

Figure 5.6. Average number of PDUs in Buffer vs Offered load for MORR allocation scheme at fixed burst.

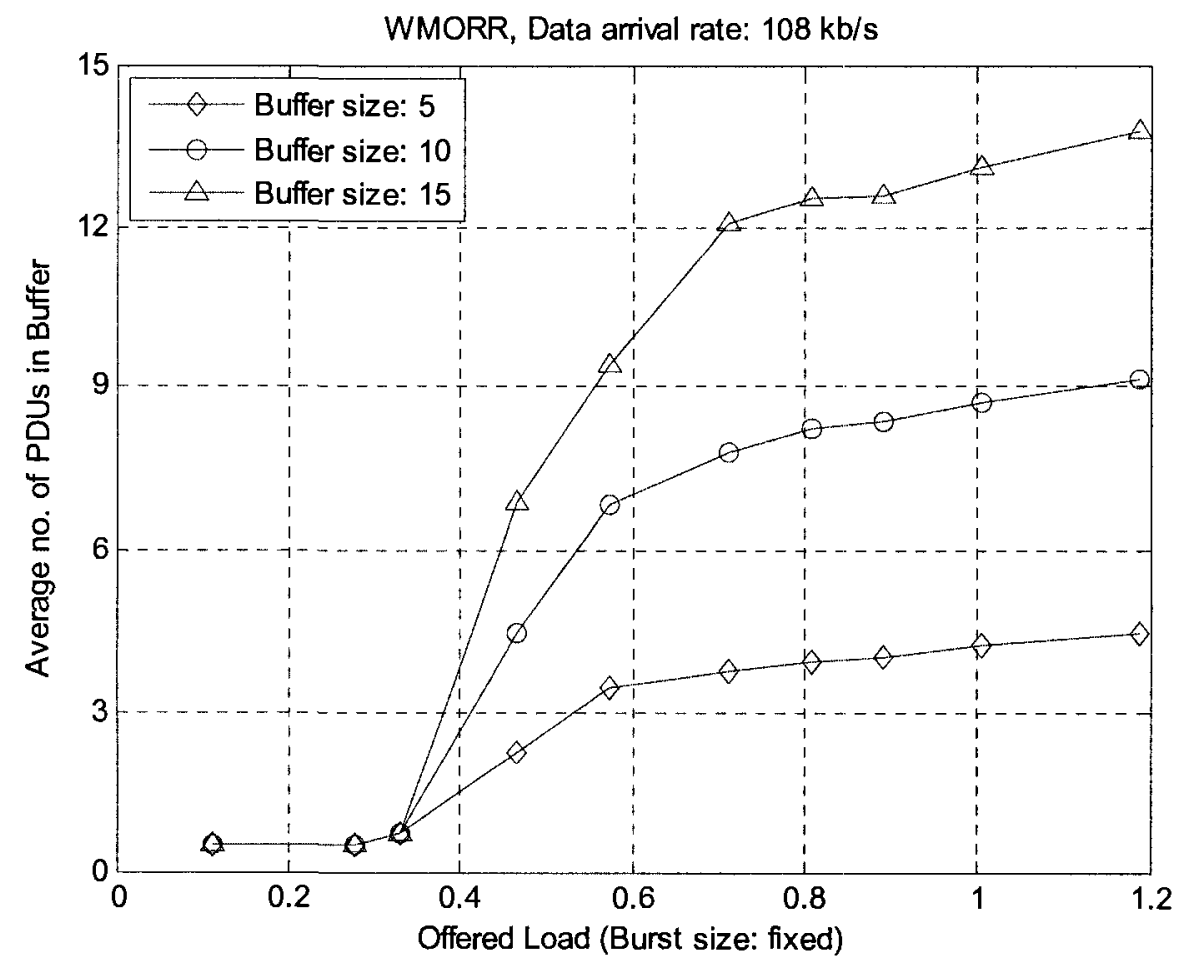

Figure 5.7. Average number of PDUs in Buffer vs Offered load for MORR allocation scheme at fixed burst. 


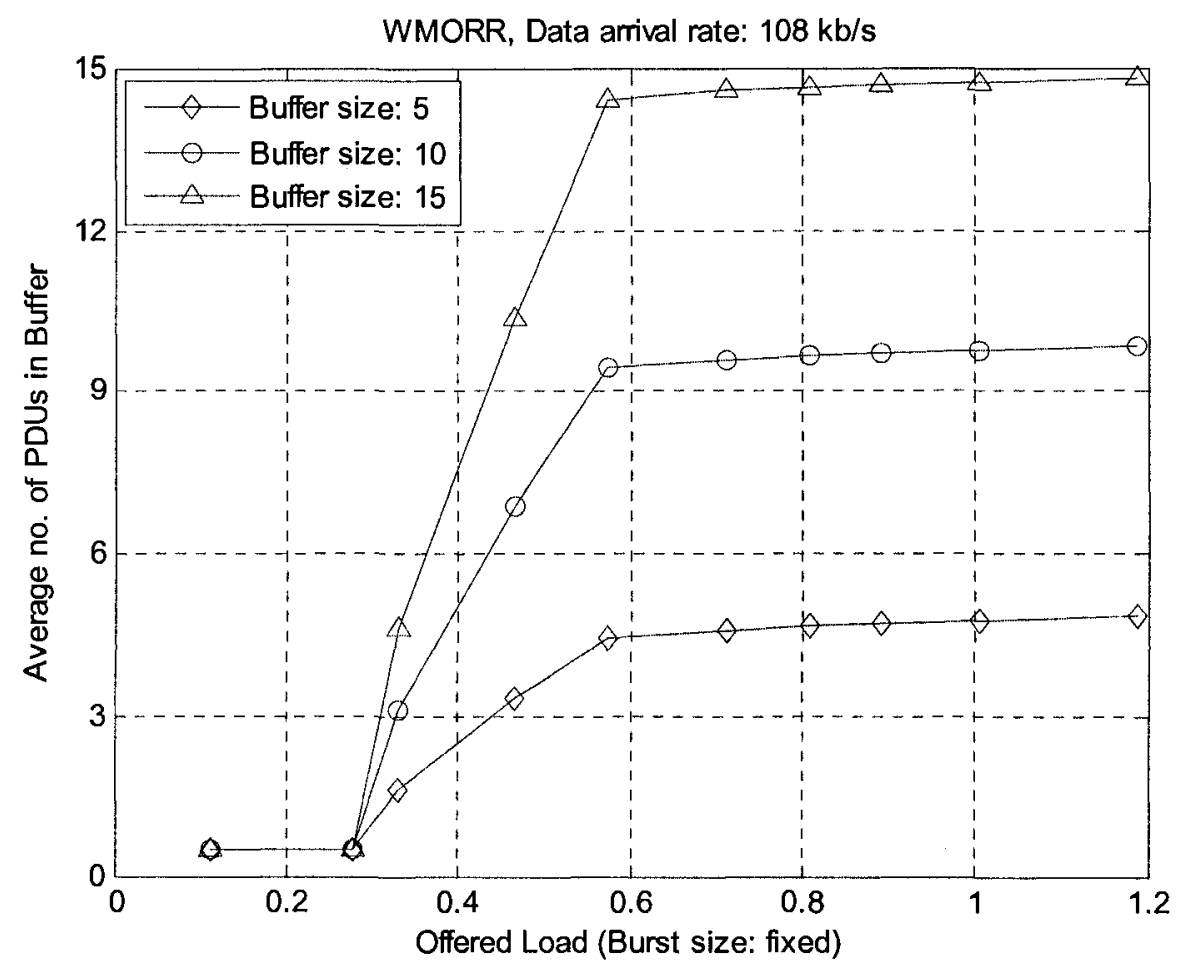

Figure 5.8. Average number of PDUs in Buffer vs Offered load for MORR allocation scheme at fixed burst.

Variance in the number of PDUs in the buffer of MTs is shown in the fig. 5.9, 5.10 and 5.11 as a function of the offered load for the three resource allocation schemes and the figures represent the results for the buffer size 5, 10 and 15 PDUs respectively. For all curves the variance of the number of PDUs in the buffer shows large changes for load values $0.3-0.8$. For very high and low value of offered load the varinace is very low and constant. This change of variance corresponds to the change in number of PDUs in the buffer (Fig. 5.2 to 5.7), where the number of PDUs in the buffer is almost constant at high and low load and it changes a lot when the offered load is bewteen 0.3 and 0.8 . It is important to notice that the curves are summetrical around the load value 0.5 becase actual carried load for the load higher than 0.5 and lower that 0.5 is the same. This indicates that our network can carry load up to 0.5 . 


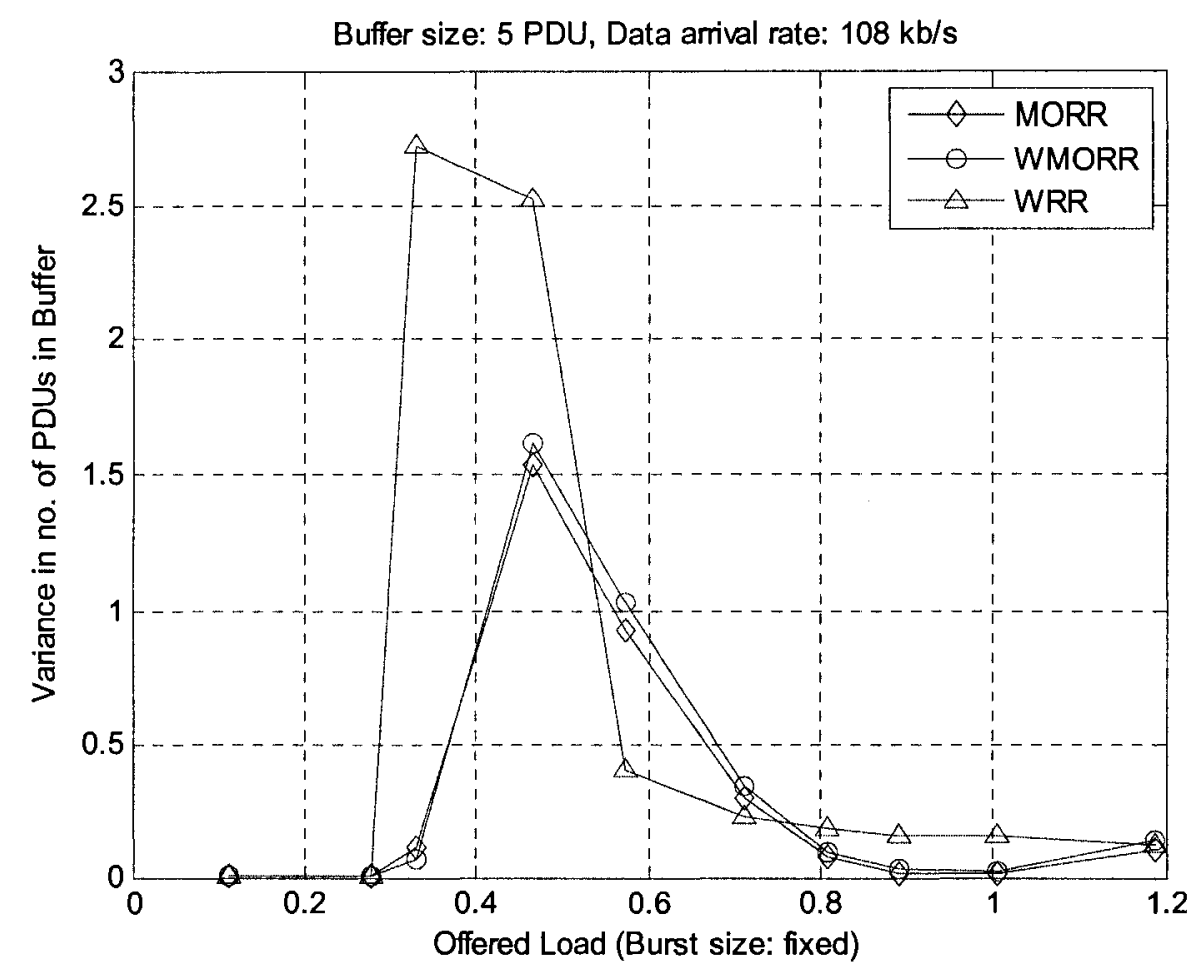

Figure 5.9. Variance in number of PDUs in Buffer vs Offered load for buffer size 5 at fixed burst.

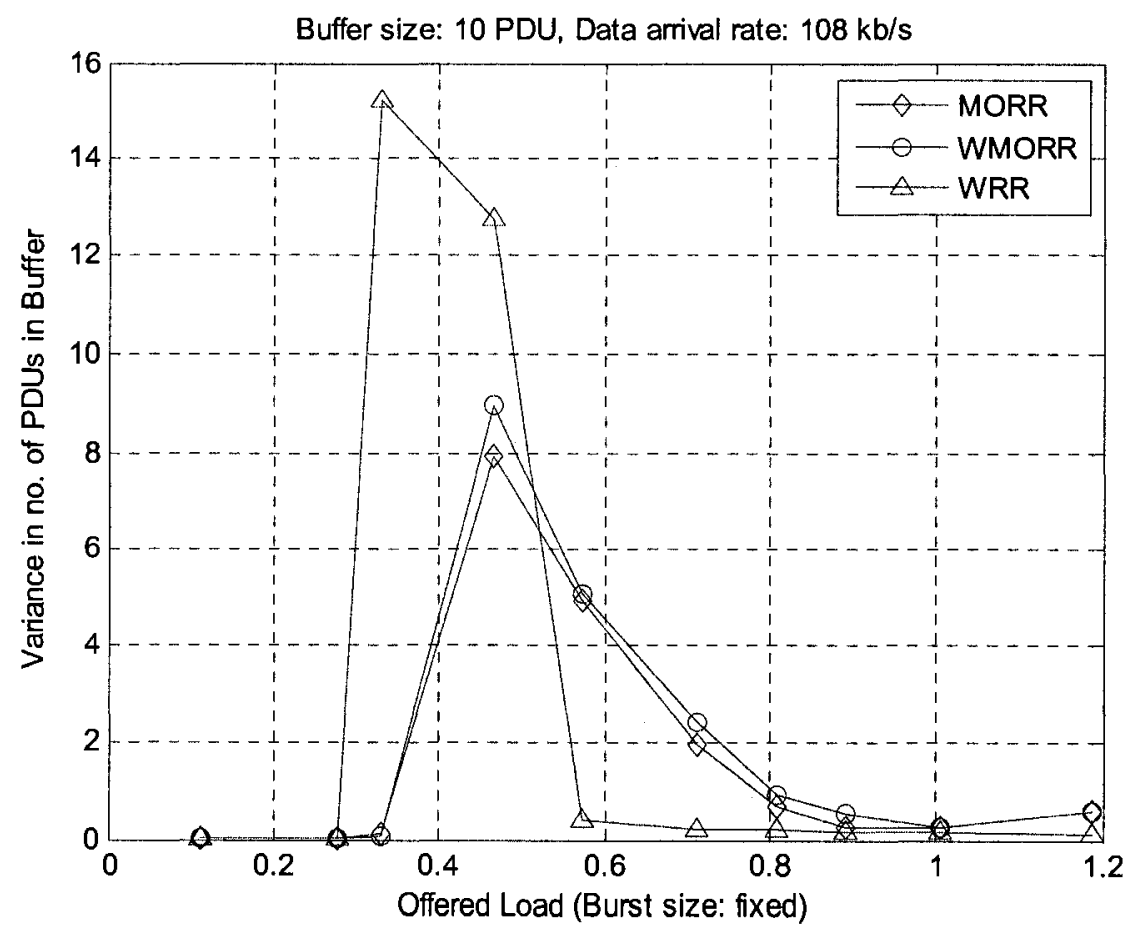

Figure 5.10. Variance in number of PDUs in Buffer vs Offered load for buffer size 10 at fixed burst. 


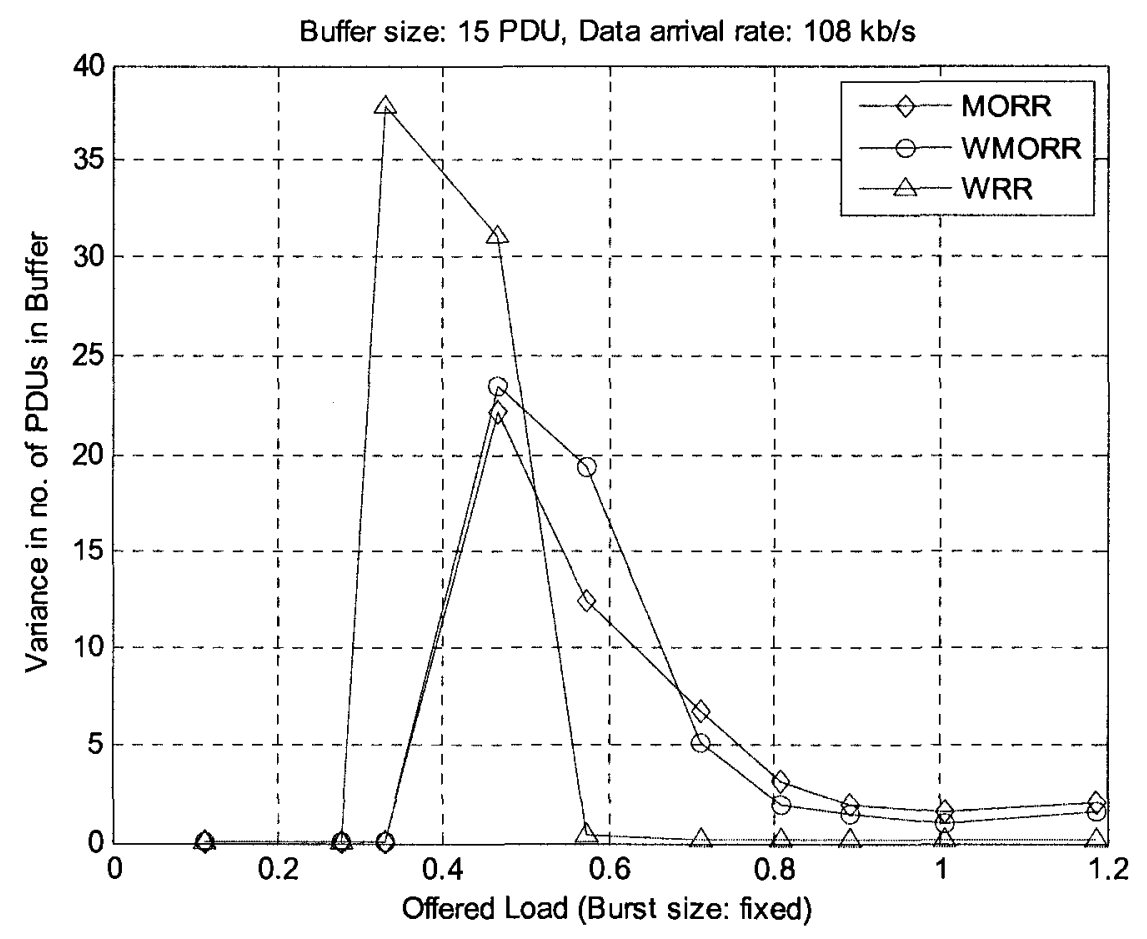

Figure 5.11. Variance in number of PDUs in Buffer vs Offered load for buffer size 5 at fixed burst.

\section{- Probability of Buffer Overflow and its variance}

Fig. 5.12, 5.13 and 5.14 depicts the probability of buffer overflow as a function of offered load for the three resource allocation policies, where buffer size is 5, 10 and 15 PDUs respectively. In figures the WMORR allocation scheme shows the lowest probability of overflow among the three schemes. It is interesting to note that the probability of overflow is zero when the load is very low and it starts increasing when the offered load is increased above 0.3 . We also notice that the probability of overflow continues to increase after this load. This value of load i.e. 0.3 is significant because at load higher than this the PDU arrival rate at our system becomes higher than the PDU service rate and the numbers of PDUs in buffer starts to increase (Fig. 5.2 to 5.7 ). 


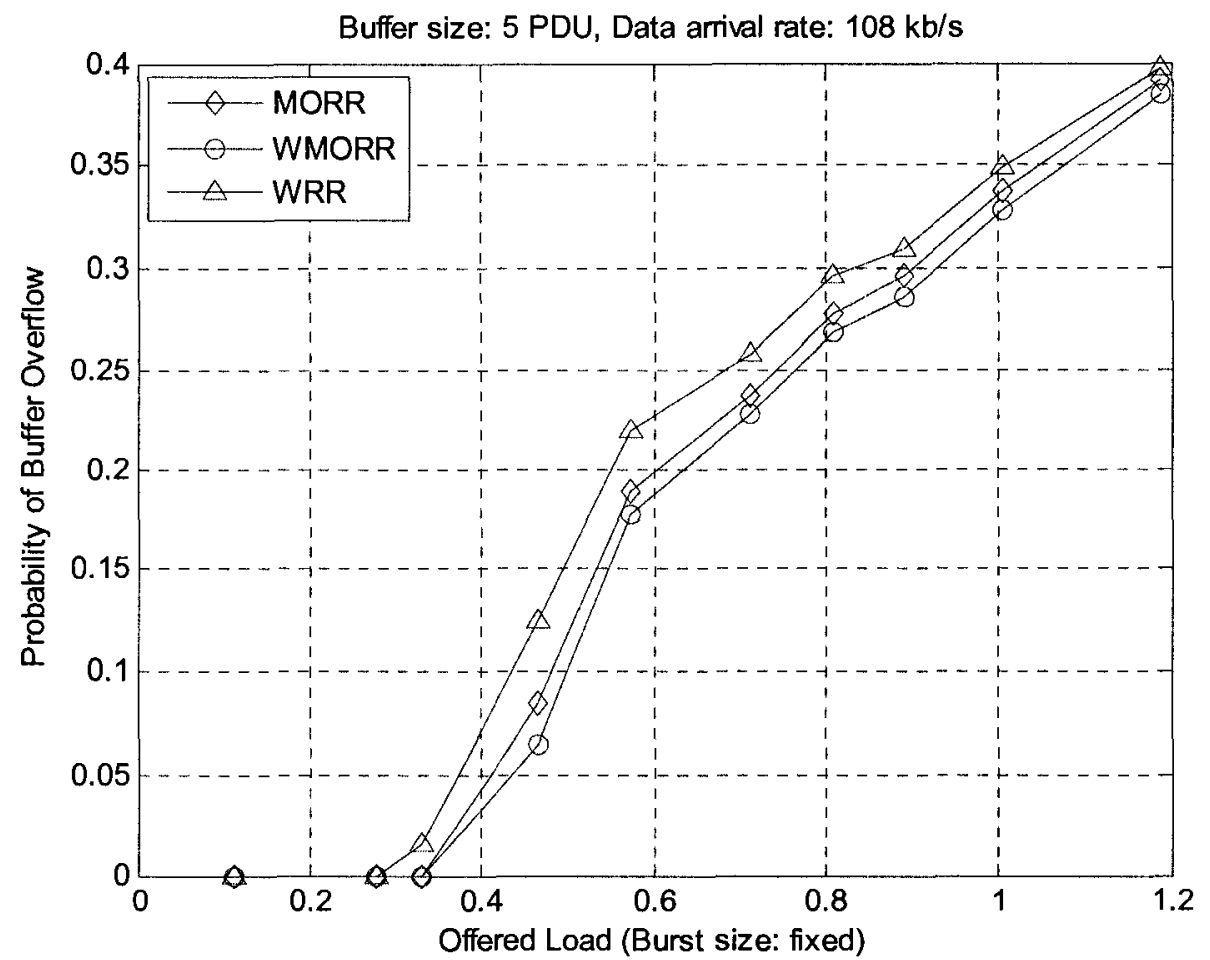

Figure 5.12. Probability of buffer overflow vs offered load for buffer size 5 at fixed burst.

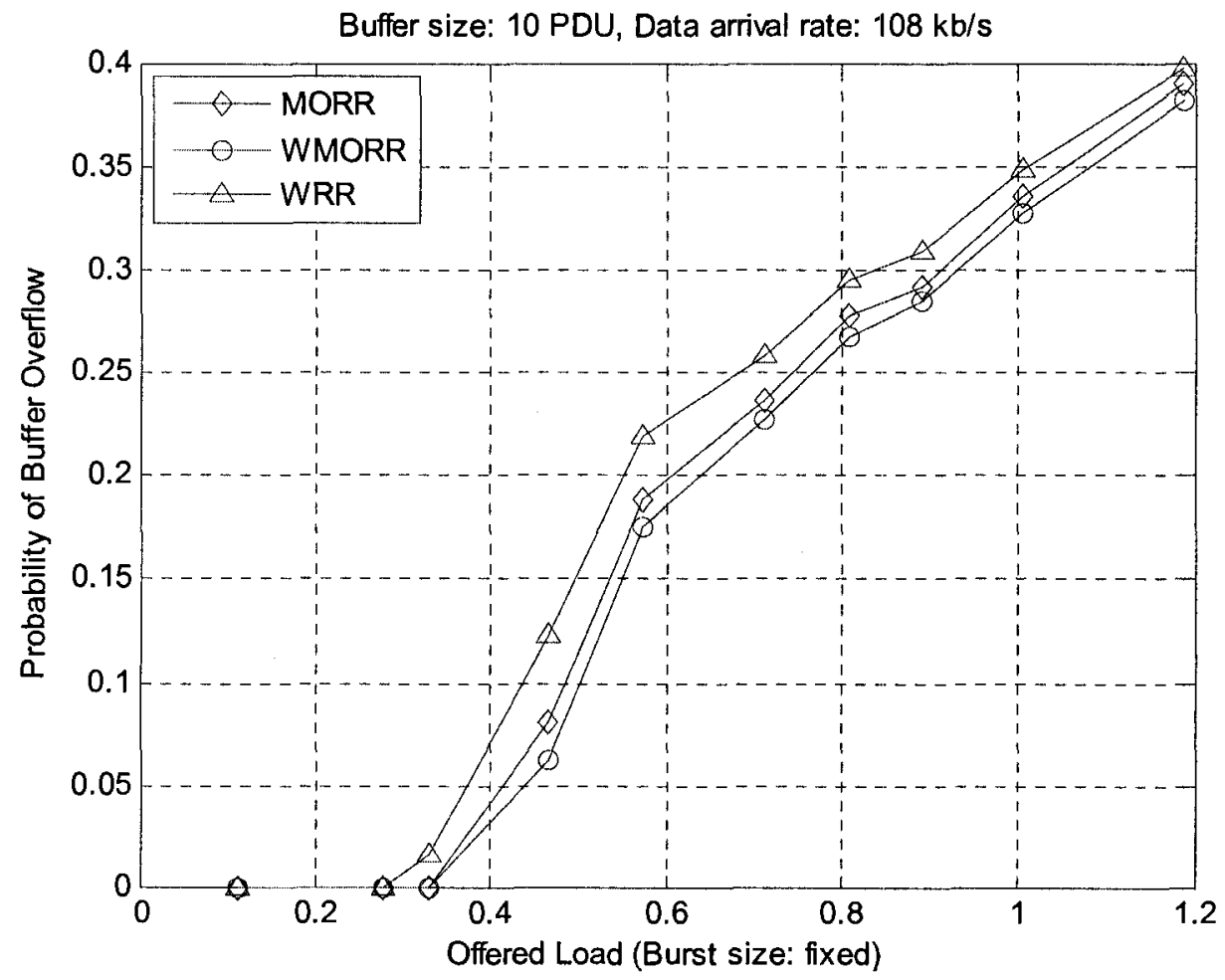

Figure 5.13. Probability of buffer overflow vs offered load for buffer size 10 at fixed burst. 


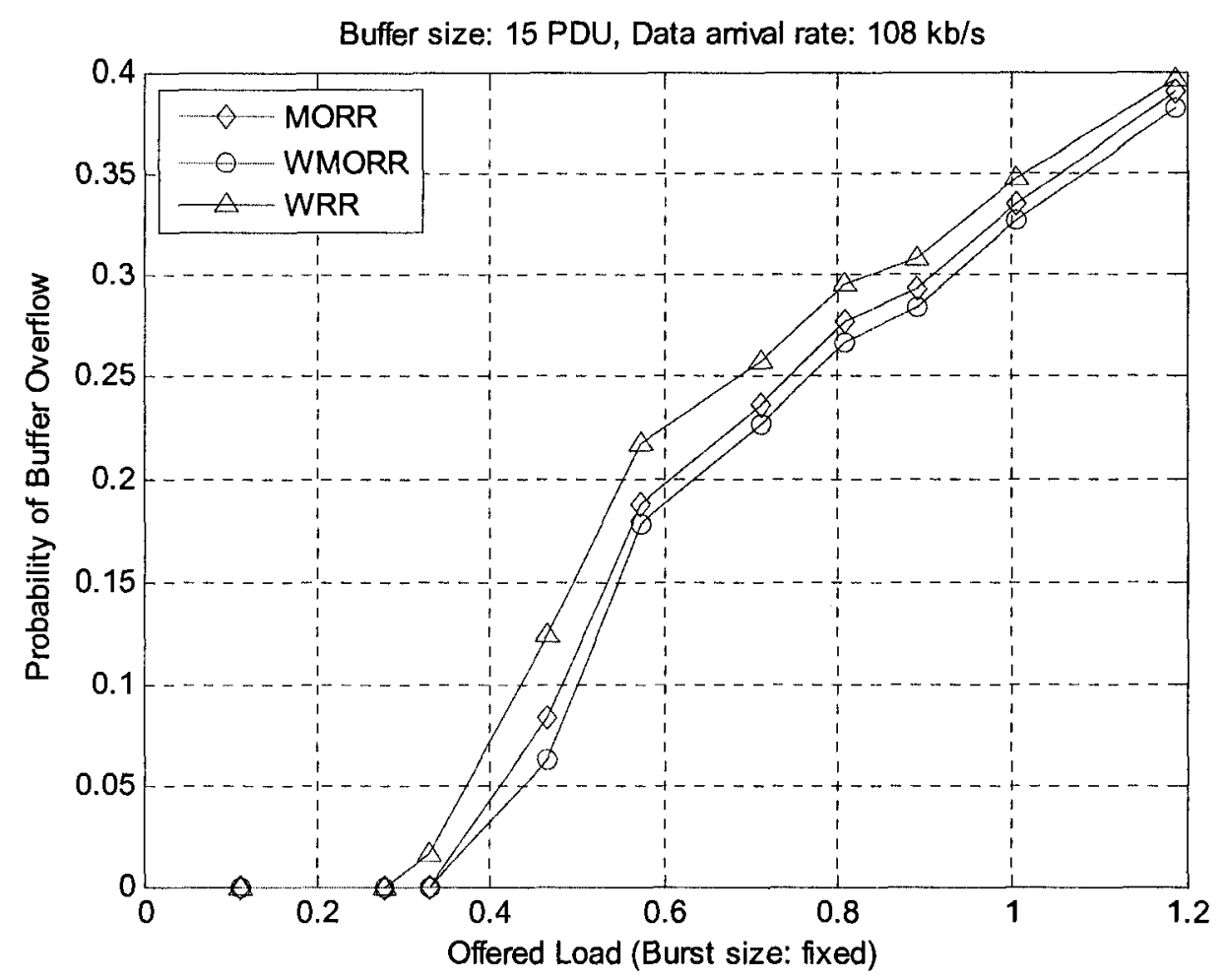

Figure 5.14. Probability of buffer overflow vs offered load for buffer size 5 at fixed burst.

Fig. 5.15, 5.16 and 5.17 shows the Probability of buffer overflow as a function of offered load for the buffer size 5, 10 and 15 PDUs and the figures are for the MORR, WMORR and WRR resource allocation schemes respectively. The figures do not show any significant change in the overflow with the increase of buffer size. It is due to large PDU arrival rate used in our simulaion. 


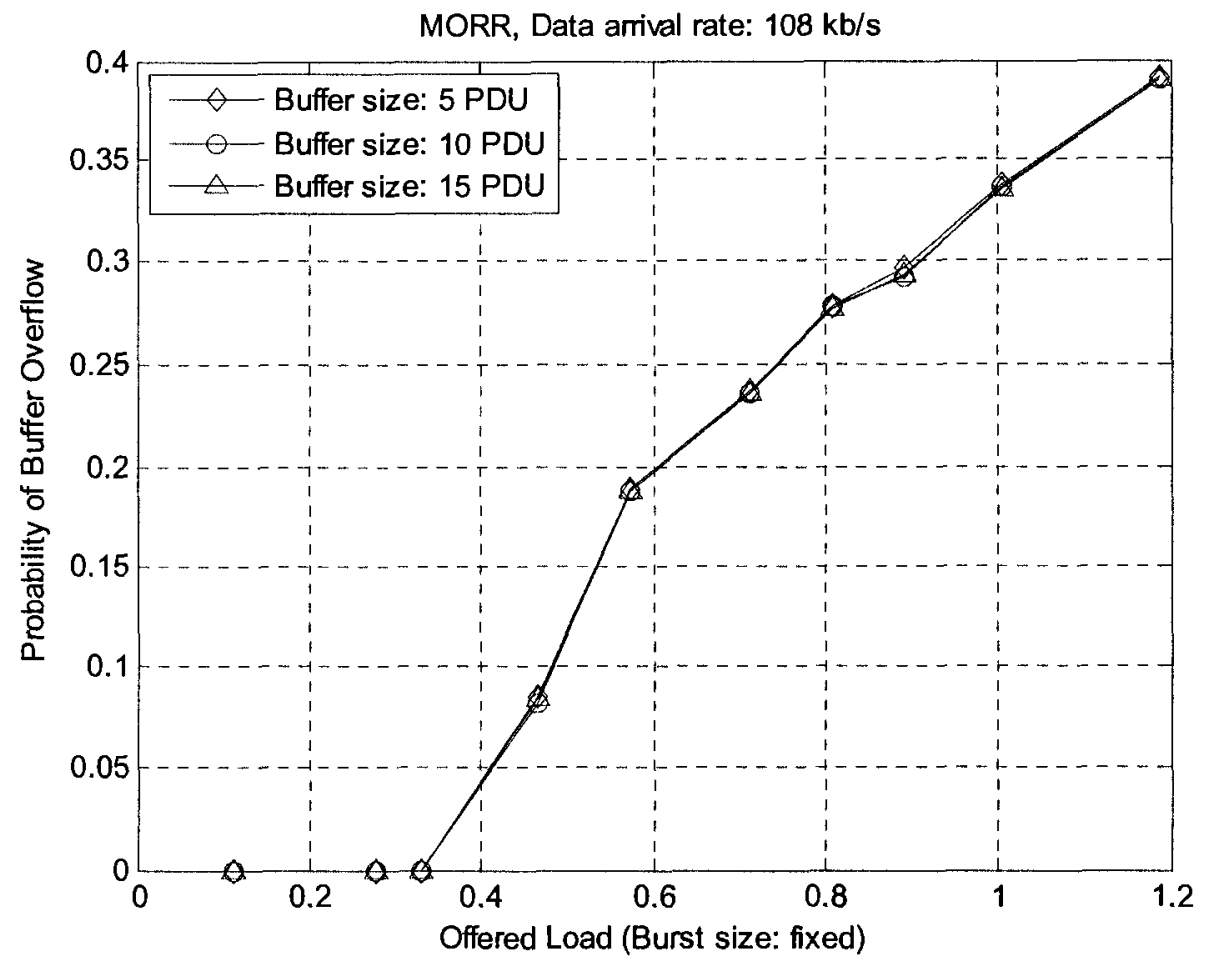

Figure 5.15. Probability of buffer overflow vs offered load for MORR allocation scheme at fixed burst.

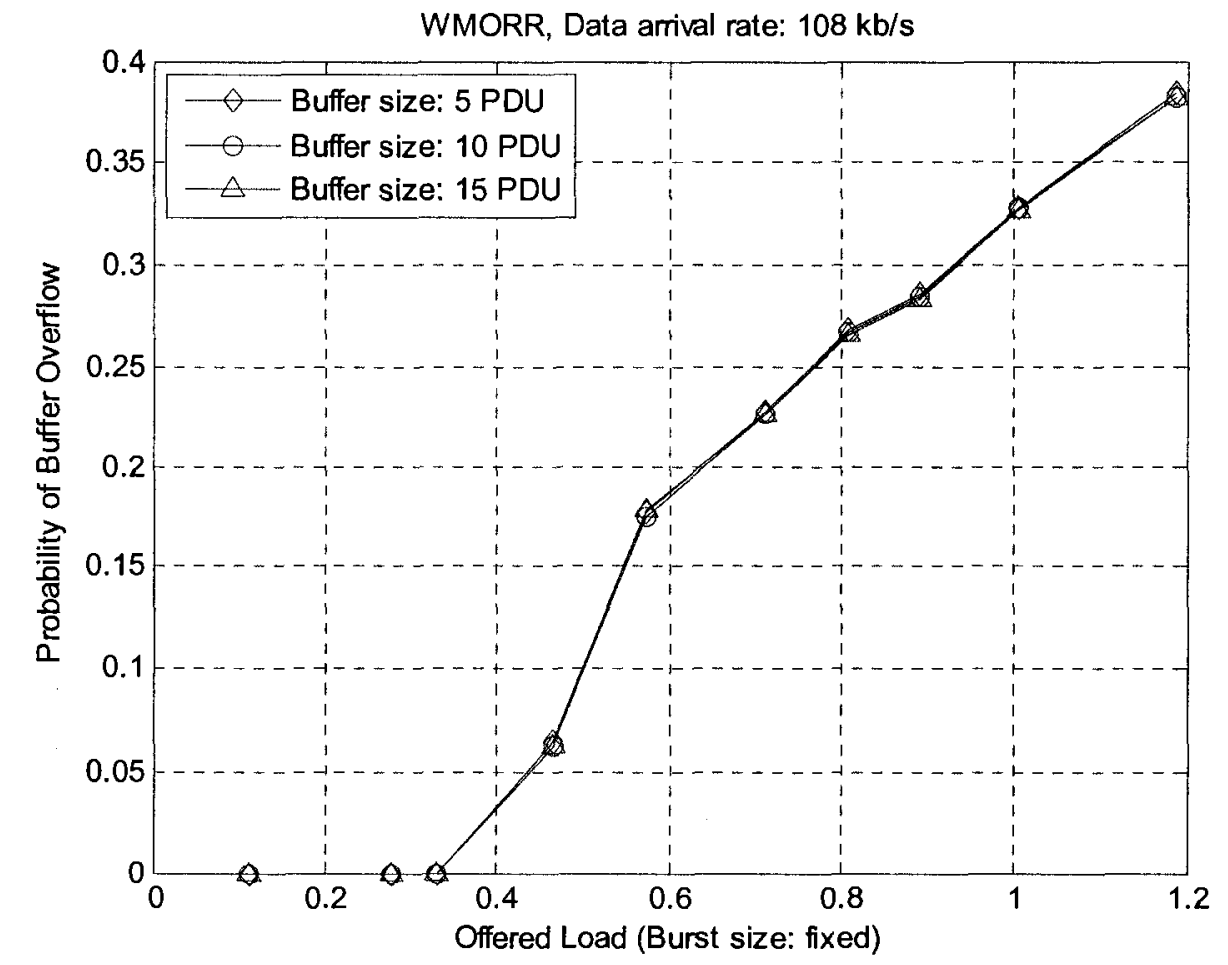

Figure 5.16. Probability of buffer overflow vs offered load for WMORR allocation scheme at fixed burst. 


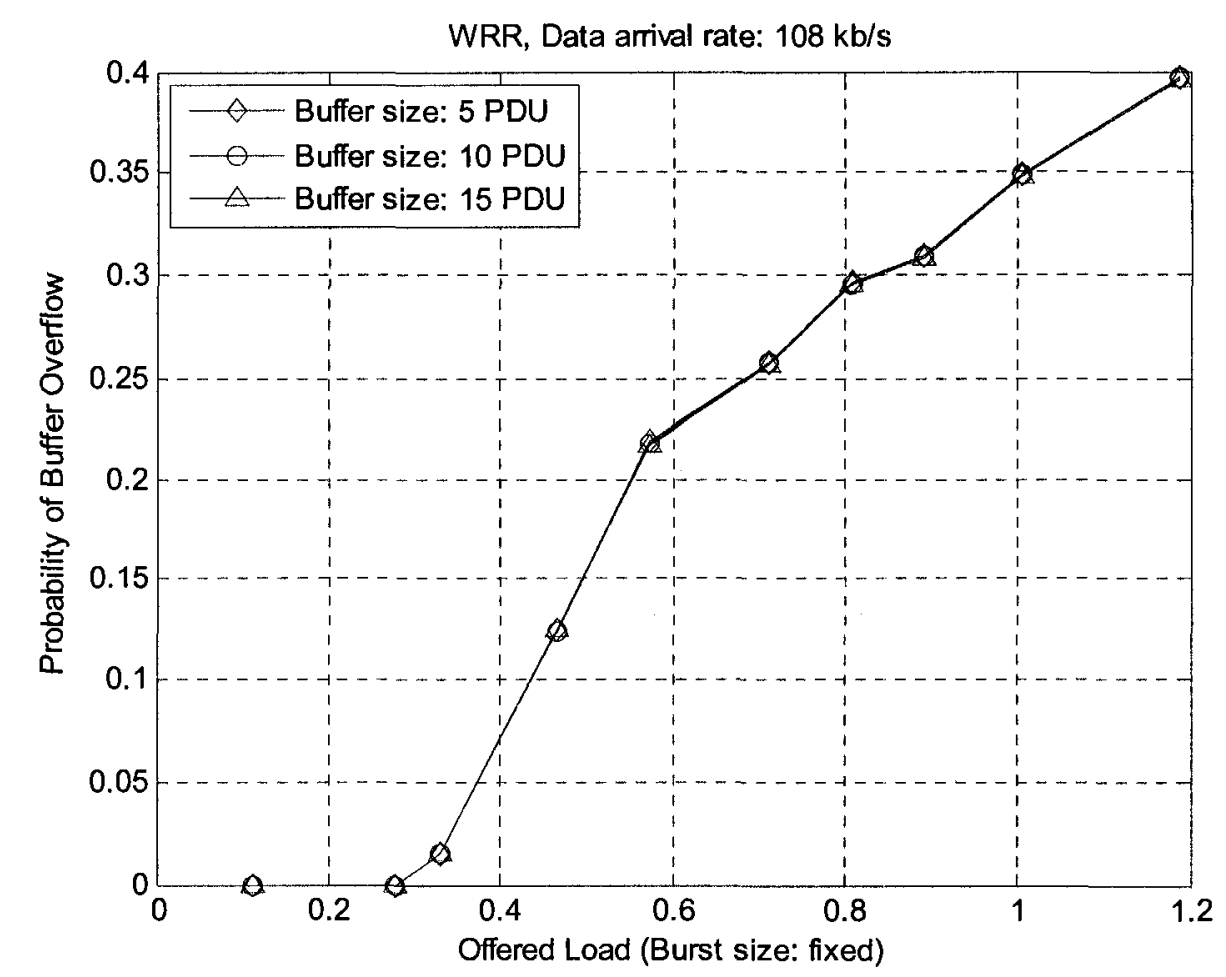

Figure 5.17. Probability of buffer overflow vs offered load for WRR allocation scheme at fixed burst.

Variance in the buffer overflow is shown in the fig. 5.18, 5.19 and 5.20 as a function of the offered load for the three resource allocation schemes and the figures represent the results for the buffer size 5, 10 and 15 PDUs respectively. For all the curves, the variance of buffer overflow is zero for low value of offered load as buffer overflow is also zero (Fig. 5.12 to 5.17). But for higer value of offered load the varinace becomes large as the change in probability of buffer overflow is large when load is high. We notice that the curves are almost symmetrical around the load value 0.5 . This is due to the fact that actual carried load is same for loads below and above of 0.5 and the network is efficient up to load value 0.5 . 


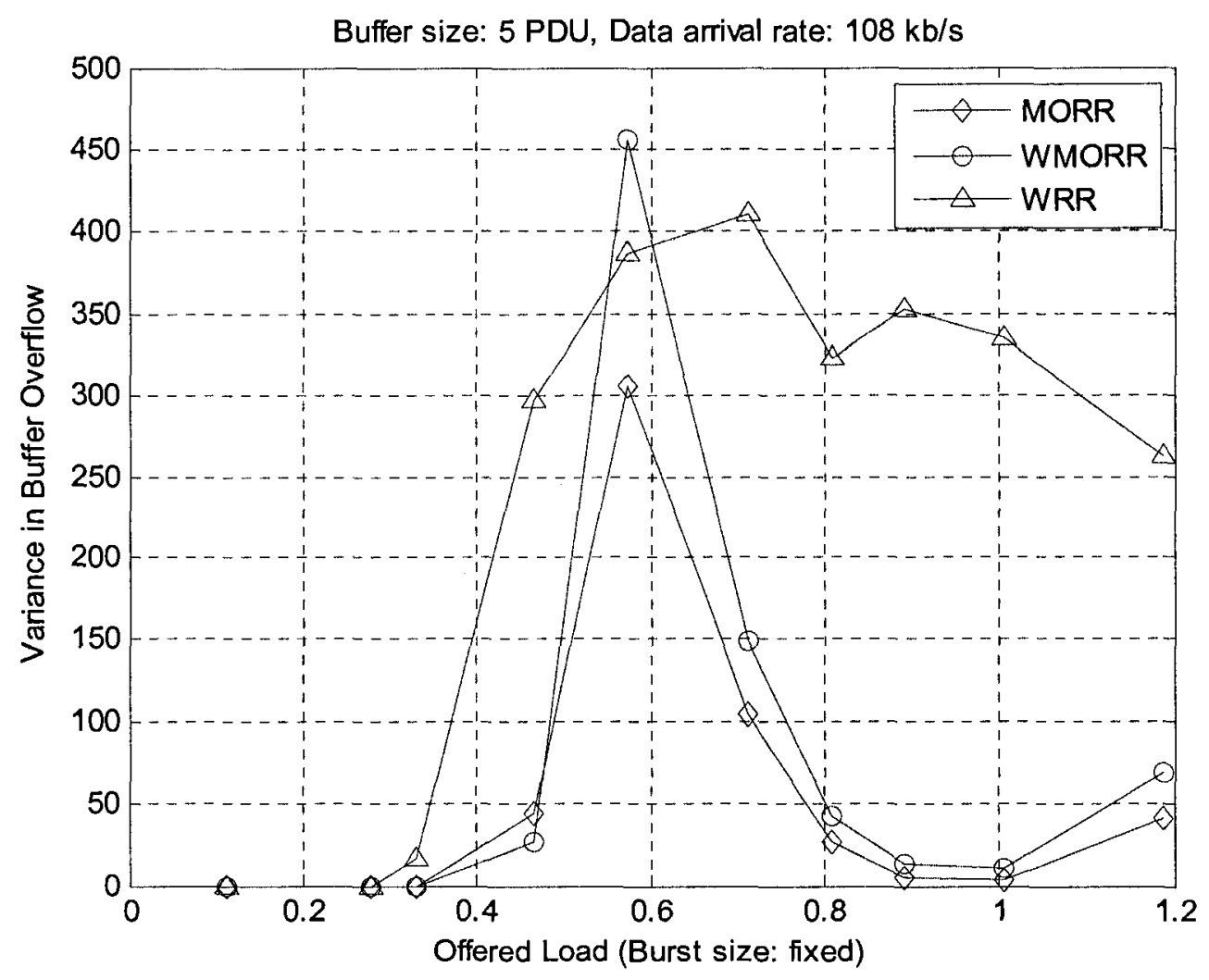

Figure 5.18. Variance in buffer overflow vs offered load for buffer size 5 at fixed burst.

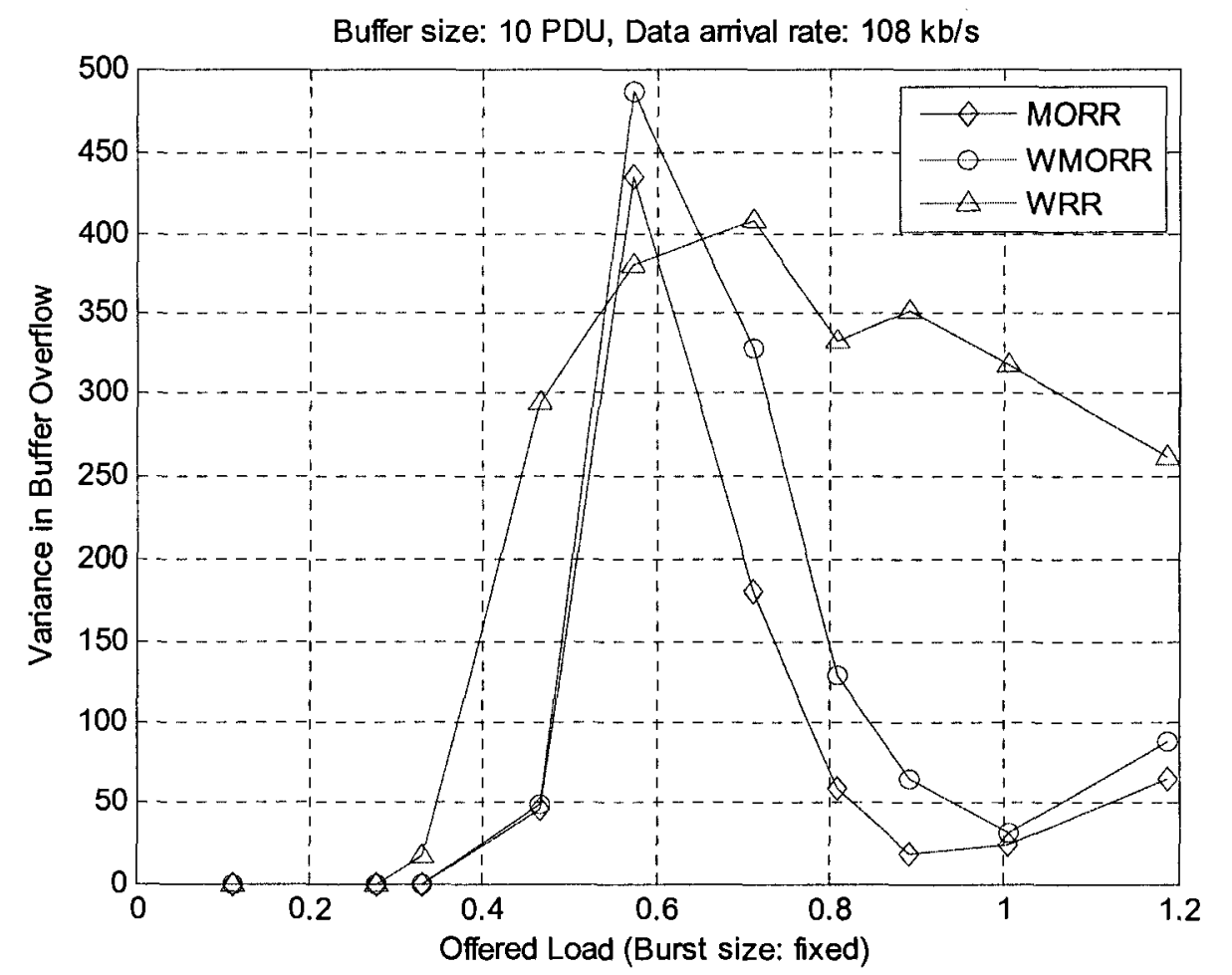

Figure 5.19. Variance in buffer overflow vs offered load for buffer size 10 at fixed burst. 


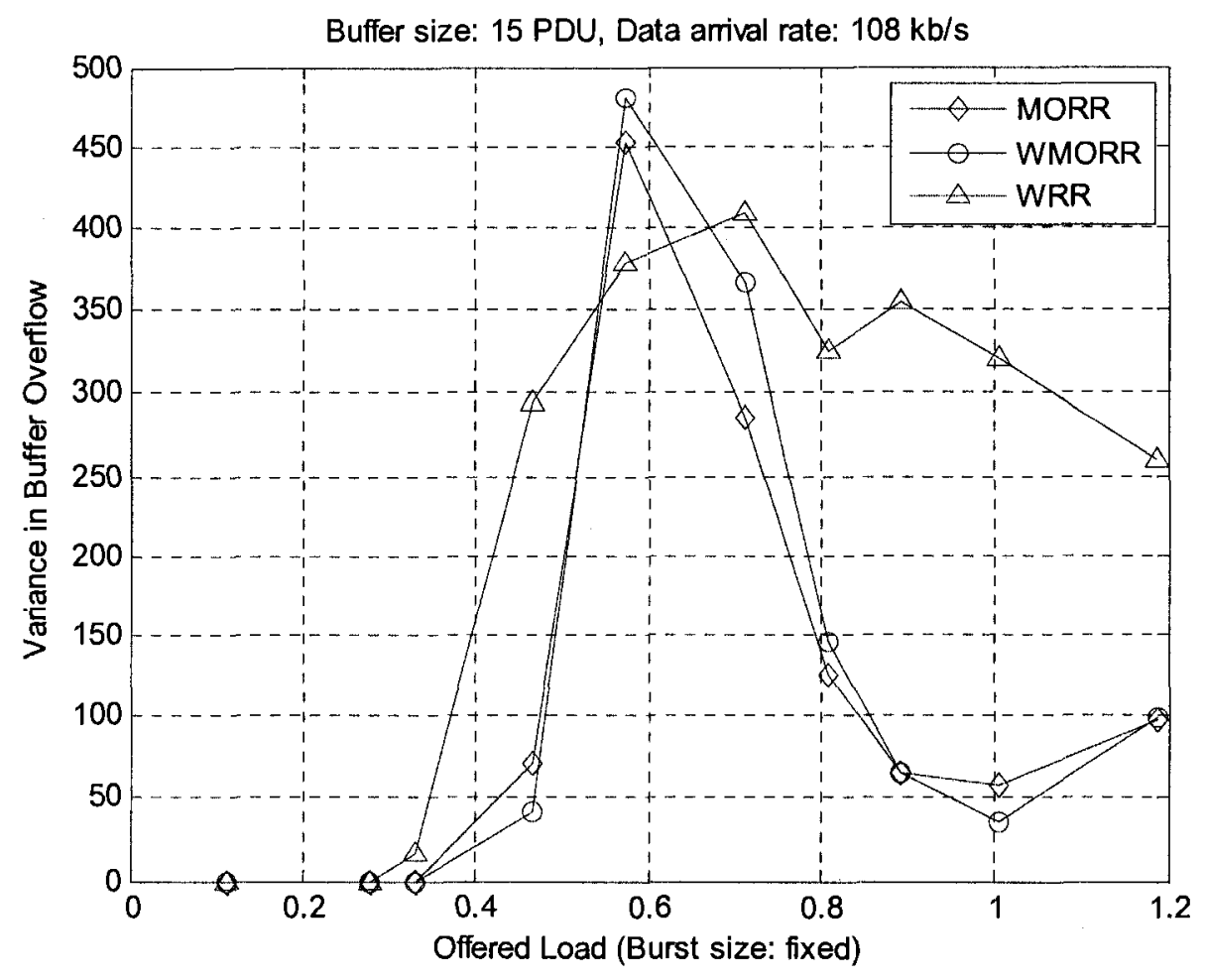

Figure 5.20. Variance in buffer overflow vs offered load for buffer size 15 at fixed burst.

\section{- Average PDU delivery time and its variance}

Fig. 5.21, 5.22 and 5.23 depicts average PDU delivery time (Seconds) as a function of offered load for the three resource allocation policies, where the buffer size is 5, 10 and 15 PDUs respectively. In the figures all the allocation policies show almost equal performance except for very high load where the WRR scheme shows less PDU delivery time compared to other allocation schemes. For low value of offerred load the delivery time is low because PDUs spend very small amount of time in the buffer. It is important to note here that for load lower than 0.3 delivery time is constant as PDUs do not have to wait in buffer for transmittion opportunity. When the offered load increases above 0.3, PDU service rate becomes lower than the arrival rate and PDUs have to wait longer in the buffer to get the transmission opportunity. 


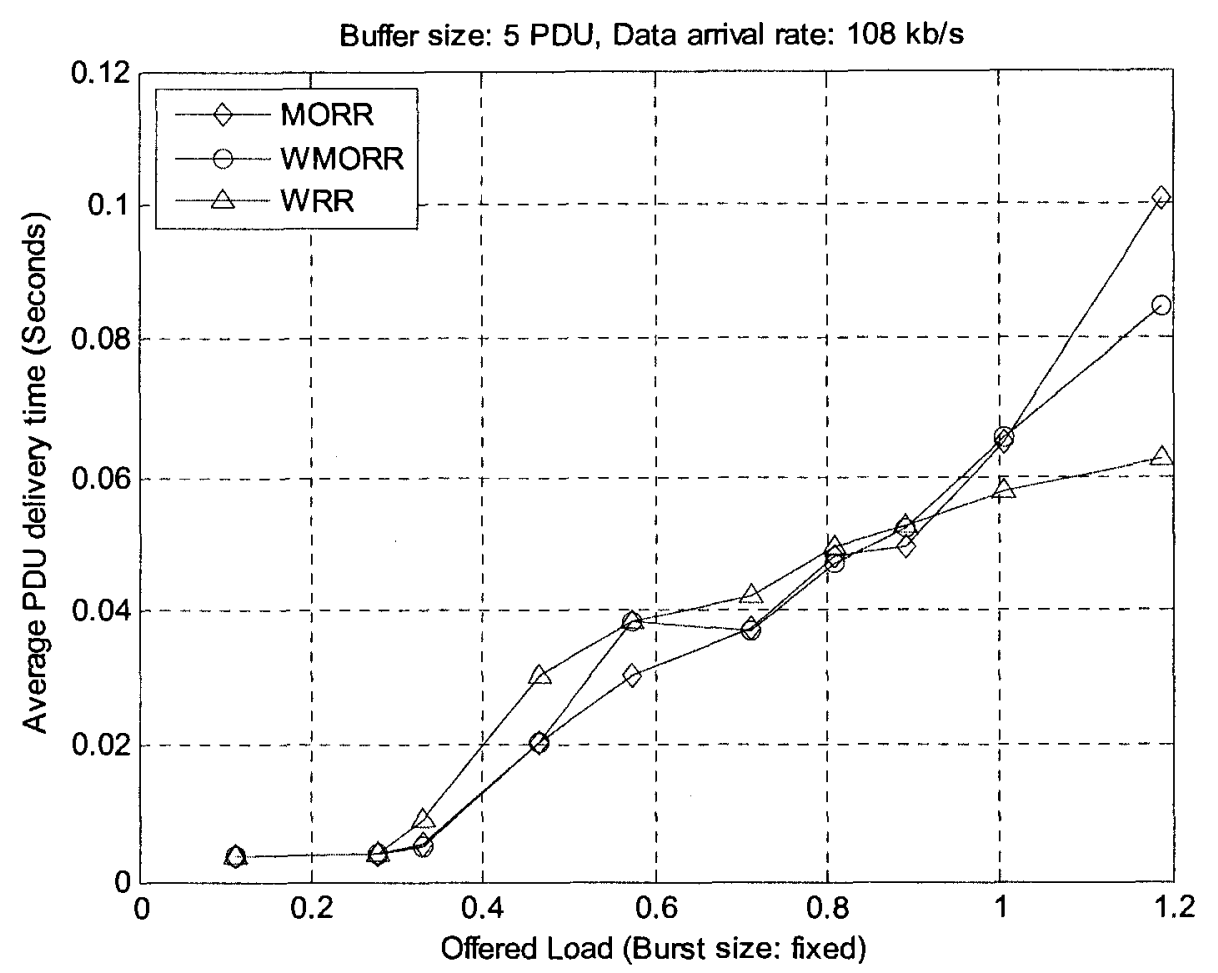

Figure 5.21. Average PDU delivery time vs offered load for buffer size 5 at fixed burst.

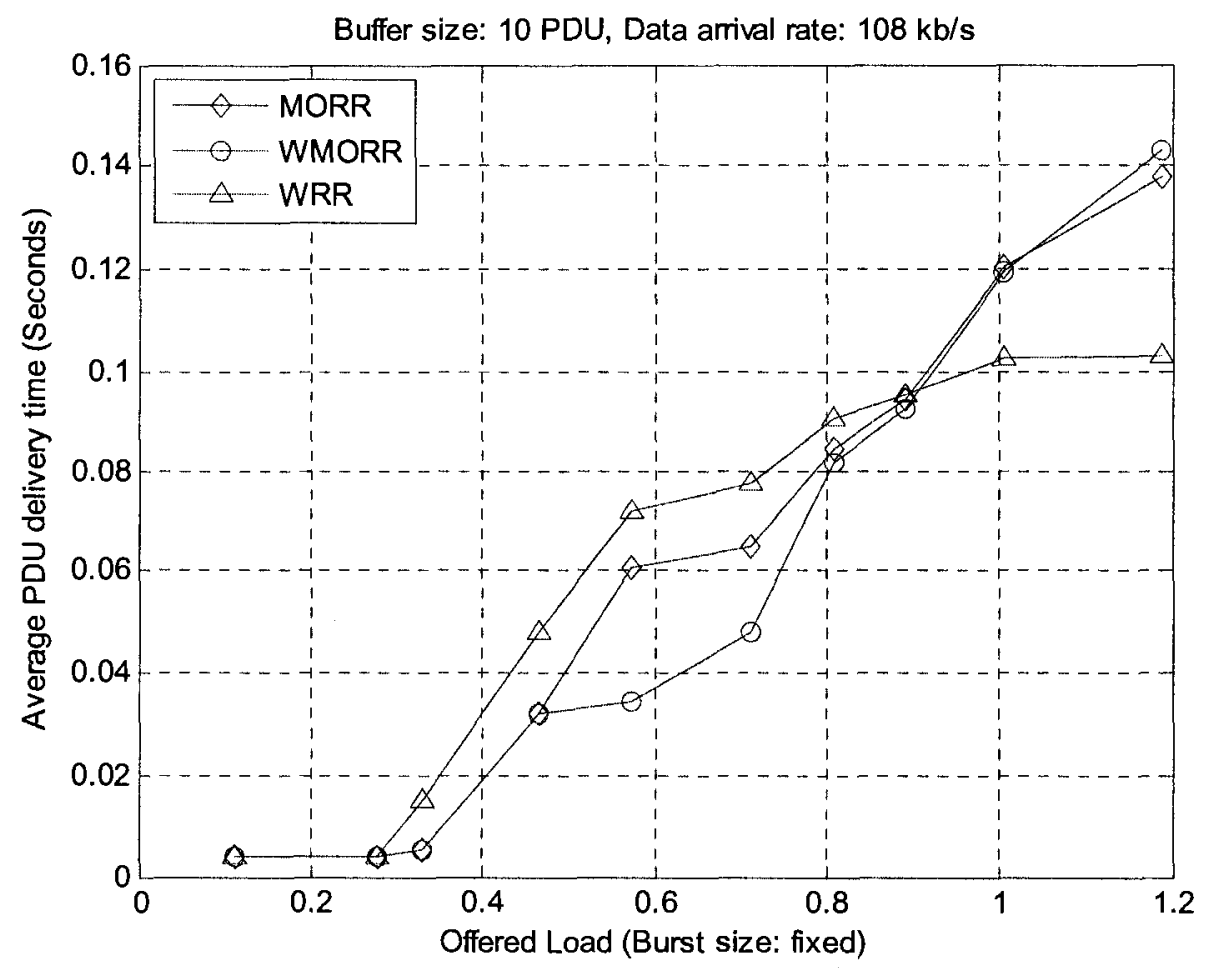

Figure 5.22. Average PDU delivery time vs offered load for buffer size 10 at fixed burst. 


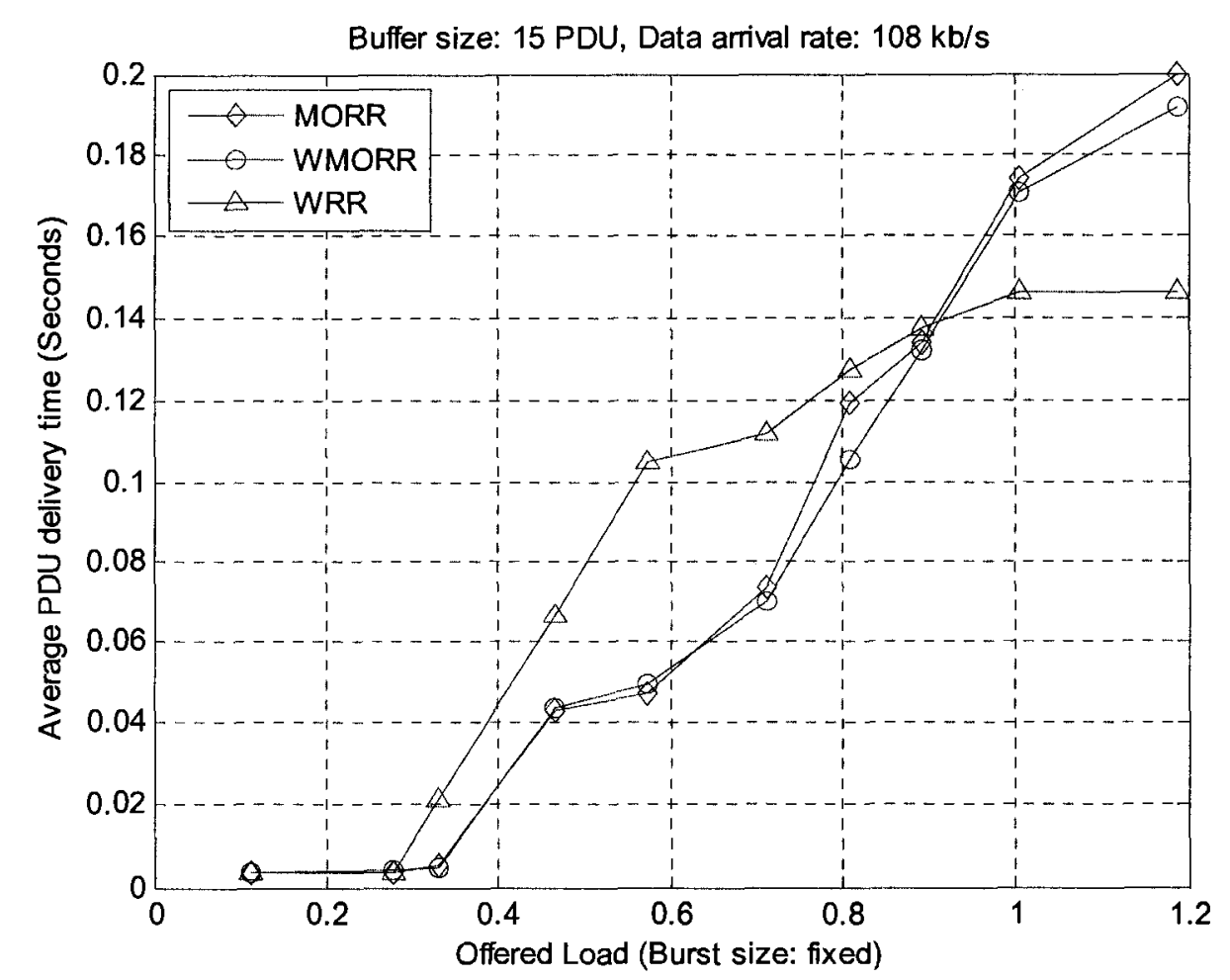

Figure 5.23. Average PDU delivery time vs offered load for buffer size 15 at fixed burst.

Fig. 5.24, 5.25 and 5.26 shows the average of PDU delivery time in seconds as a function of offered load for buffer sizes 5, 10 and 15 PDUs and the figures are for the MORR, WMORR and WRR resource allocation schemes respectively. All the figures show that with the increase of buffer size average delivery time also increases as PDUs have to wait longer in the buffer to get the transmission opportunity. Also in these figures we notice that for offered load lower than 0.3 PDU delivery time does not depend on buffer size. 


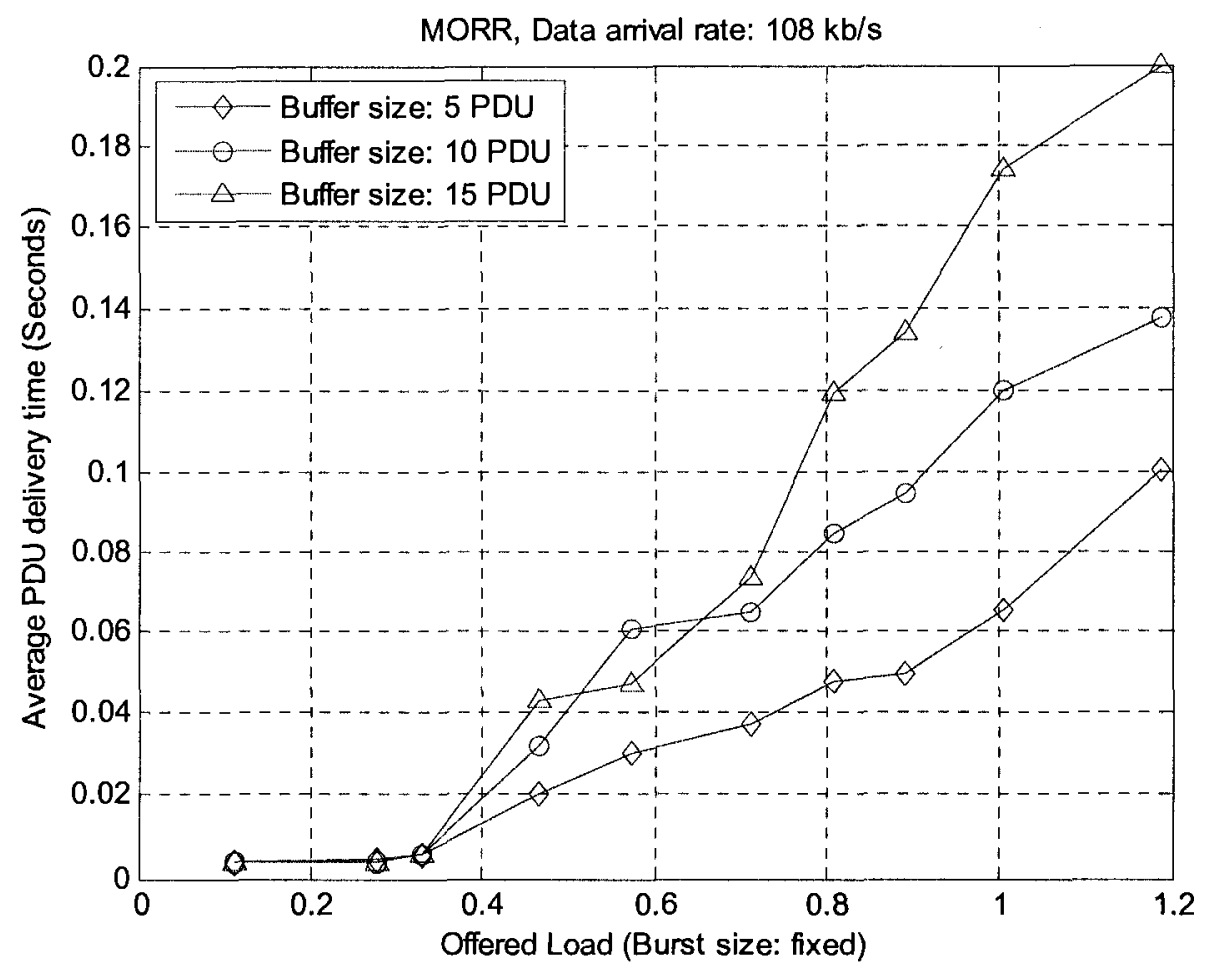

Figure 5.24. Average PDU delivery time vs offered load for MORR allocation scheme at fixed burst.

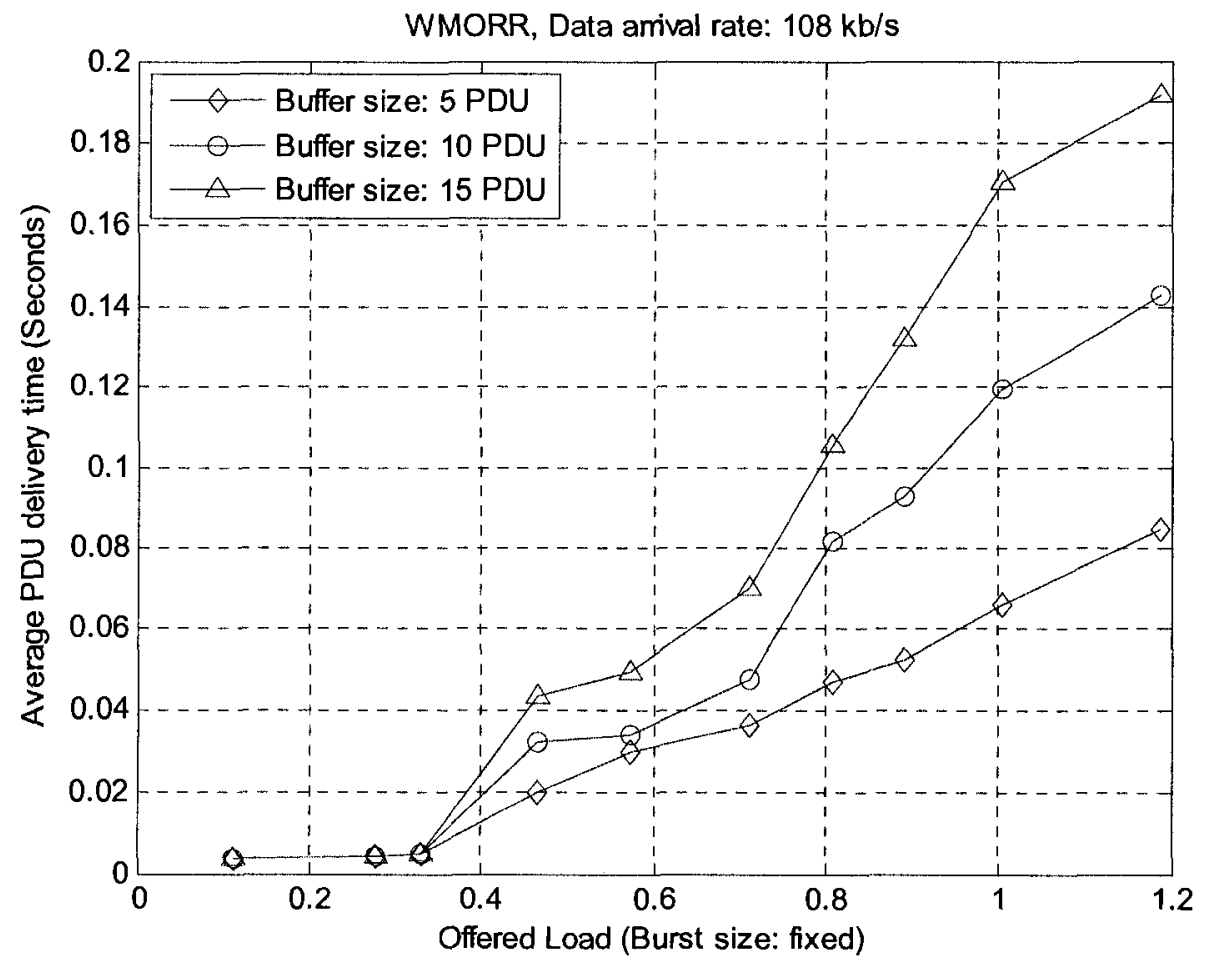

Figure 5.25. Average PDU delivery time vs offered load for WMORR allocation scheme at fixed burst. 


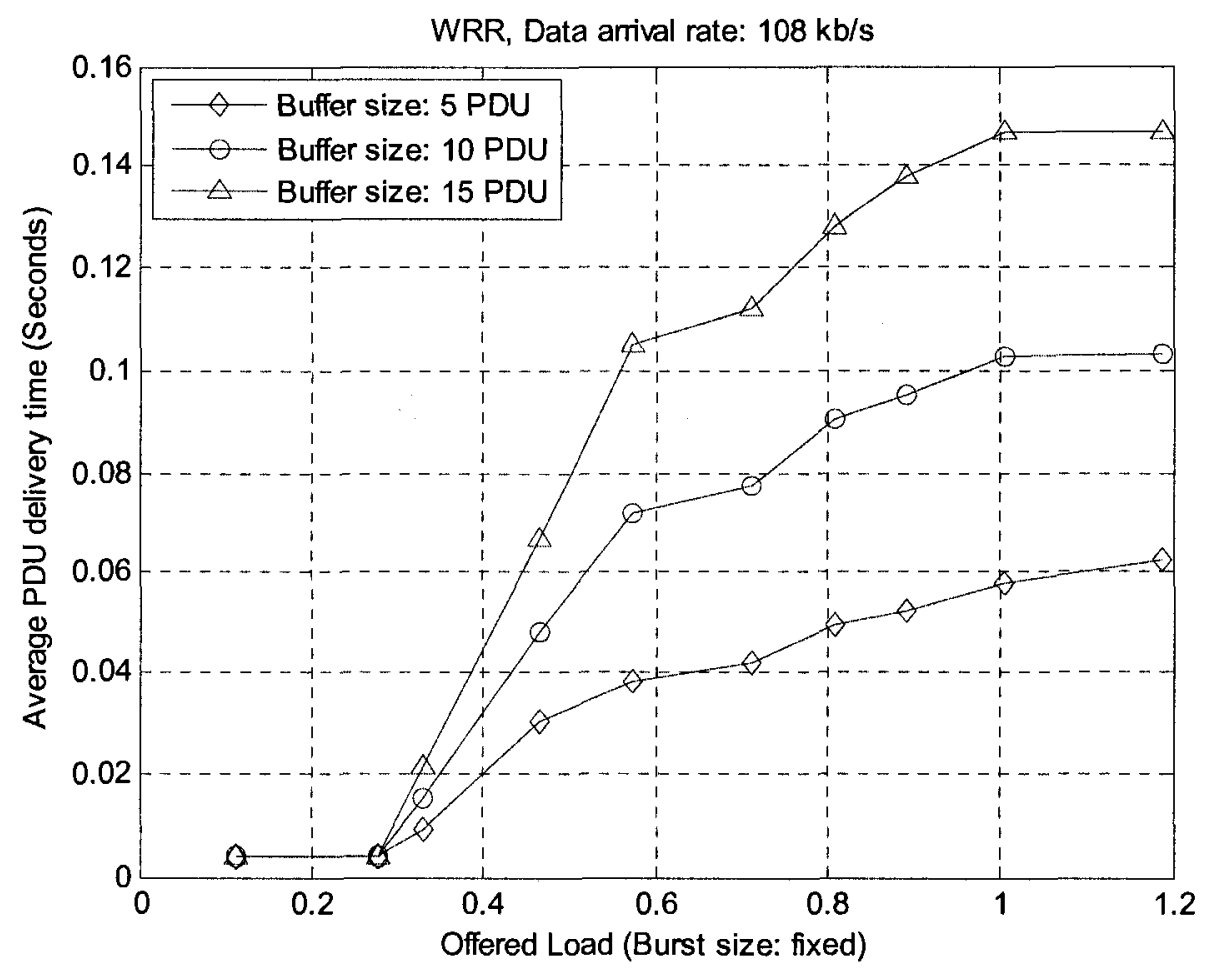

Figure 5.26. Average PDU delivery time vs offered load for WRR allocation scheme at fixed burst.

Variance in the PDU delivery time is shown in the fig. 5.27, 5.28 and 5.29 as a function of offered load for the three resource allocation schemes and the figures gives the results for the buffer size 5, 10 and 15 PDUs respectively. For all the curves the variance of PDU delivery time is zero for offered load lower than 0.3 as PDU delivery time is almost constant (Fig. 5.21 to 5.26) around these loads. But for higer value of offered load variance in delivery time becomes high. We also notice that the WRR scheme has higher variance than the other two allocation scehmes. 


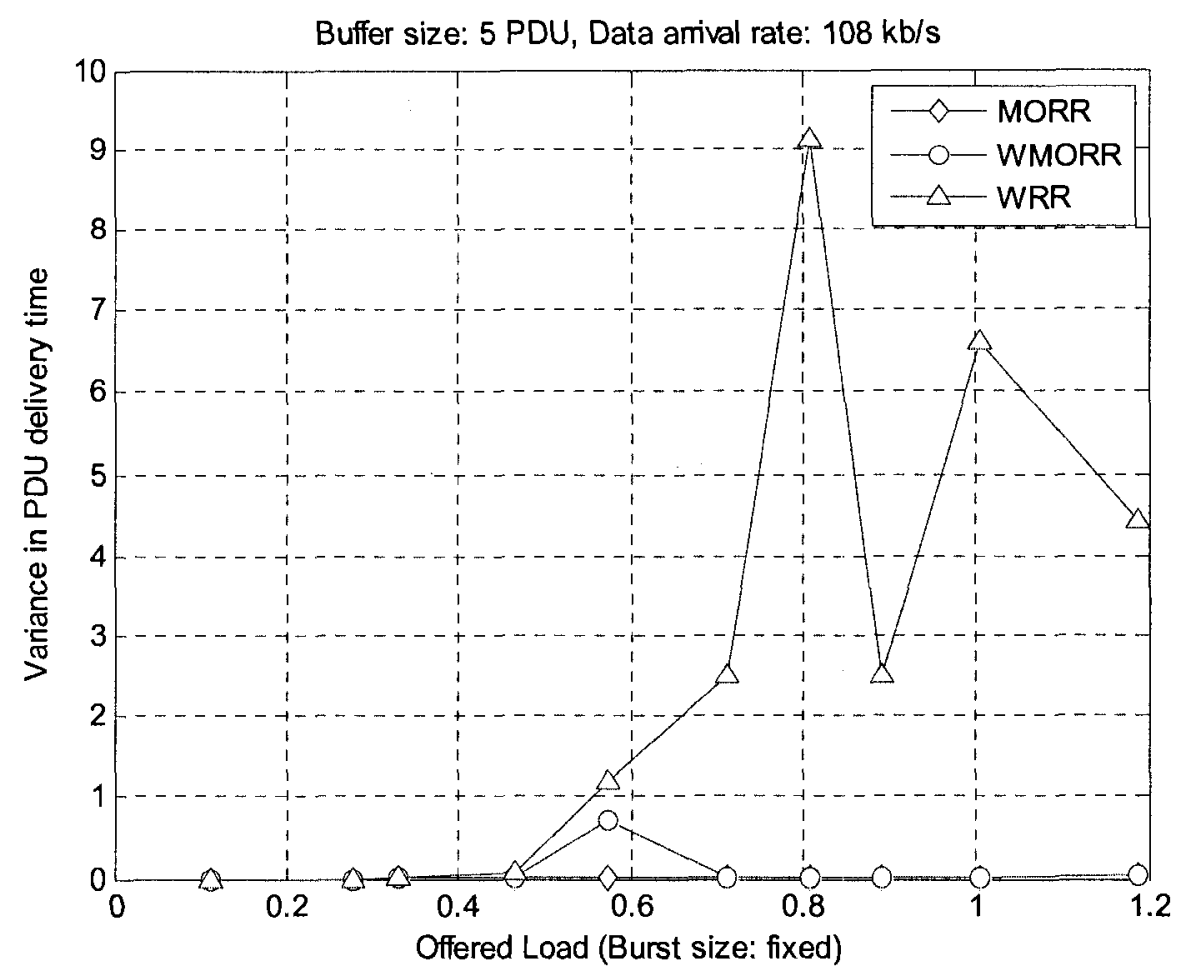

Figure 5.27. Variance in PDU delivery time vs offered load for buffer size 5 at fixed burst.

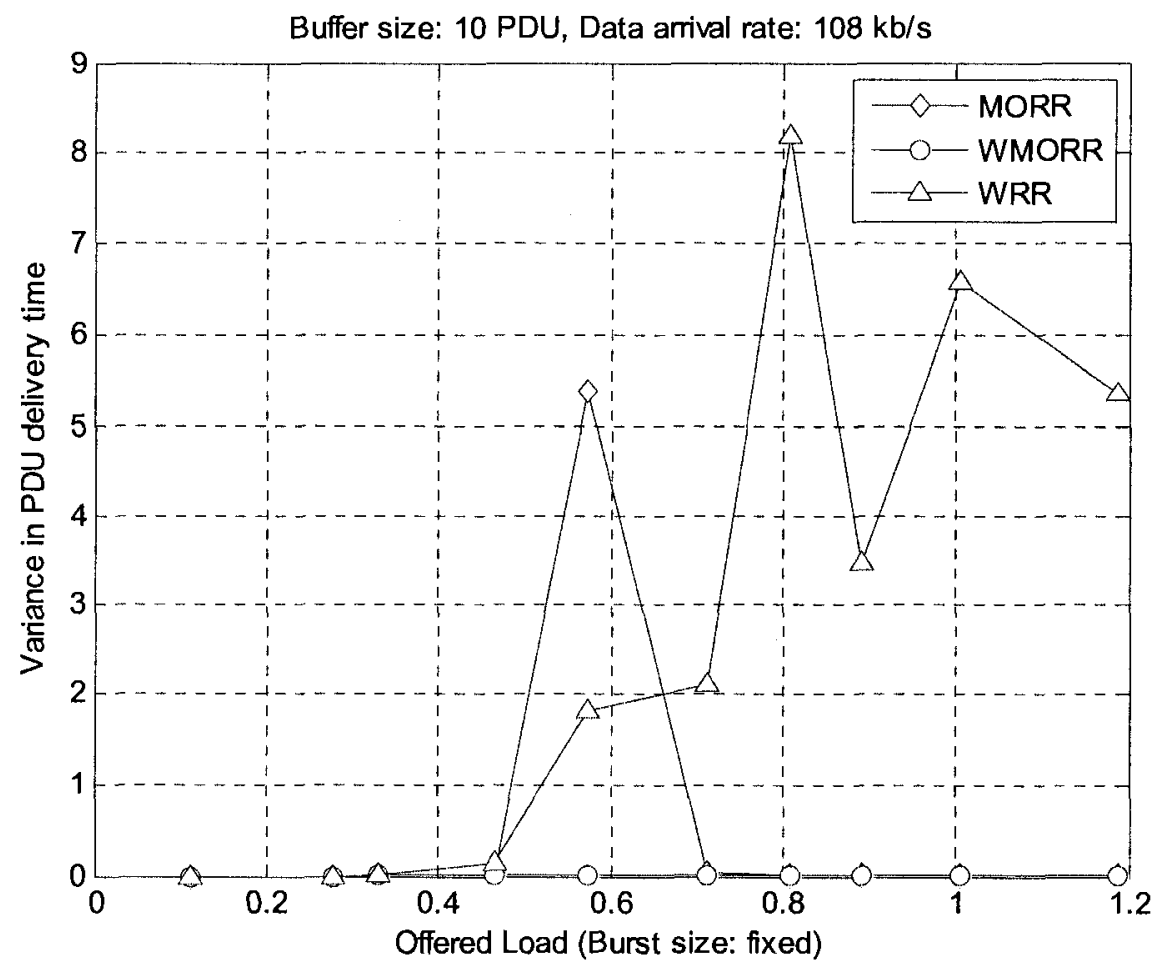

Figure 5.28. Variance in PDU delivery time vs offered load for buffer size 10 at fixed burst. 


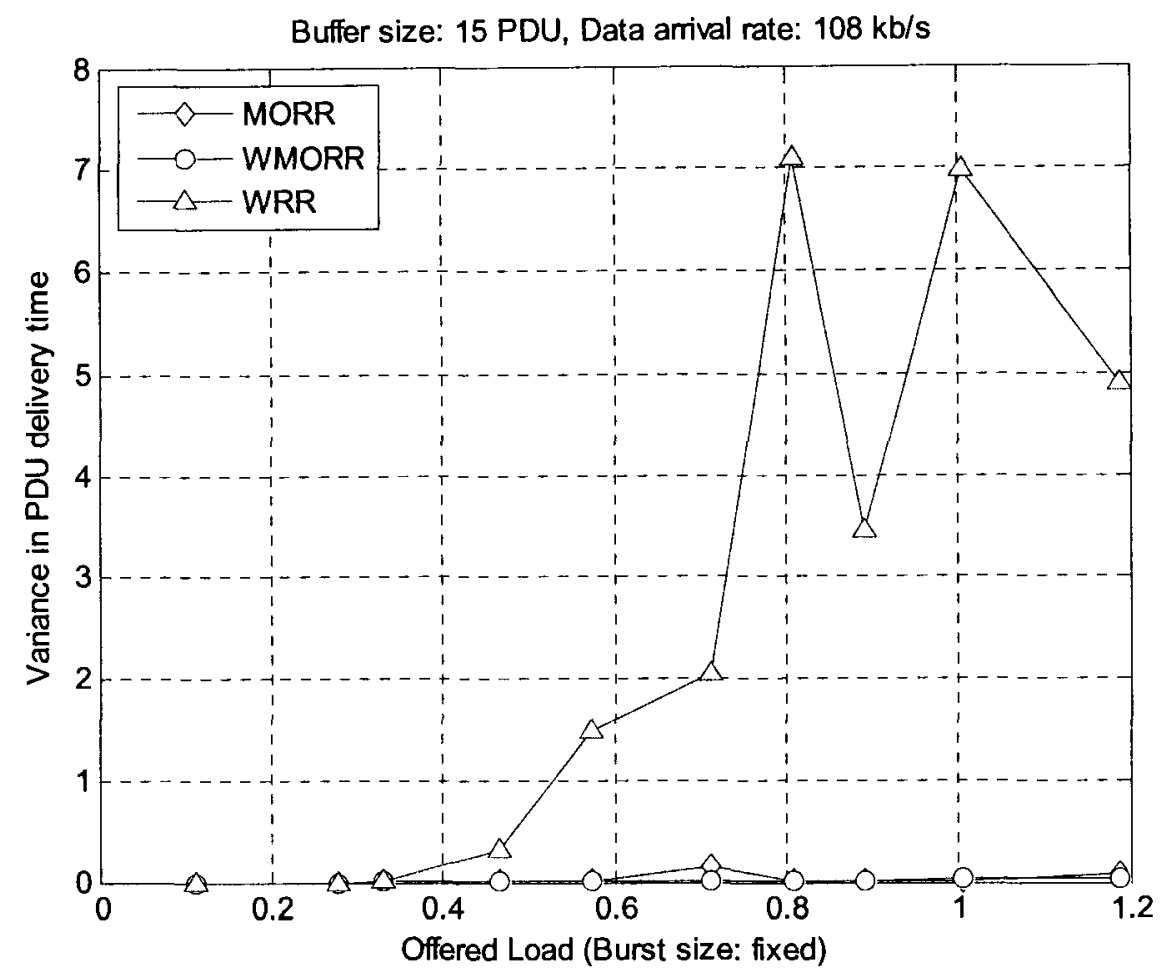

Figure 5.29. Variance in PDU delivery time vs offered load for buffer size 15 at fixed burst.

\section{- Average overhead in Uplink phase and its variance}

Fig. 5.30, 5.31 and 5.32 depict the average overhead (Percentage of total uplink time) in uplink phase as a function of offered load for the three resource allocation policies, where the buffer size is 5, 10 and 15 PDUs respectively. These figures show that the MORR and WMORR policies have low overhead compared to the WRR allocation scheme. For low value of offered load the uplink overhead is large as all the arrived PDU gets the transmission oppourtunity and overhead of all the user add up to give a large overhead. But as the load increases arrival rate beomes higher than the service rate and very few of the user get the transmission opportunity. This results very low overhead for MORR and WMORR scheme as whenever an user is visited, maximum capacity is assigned to it in these schemes. But for the WRR scheme as all the user buffer is visited during a TDMA frame, ovherhead of all the user adds up and the summaion continue to increase with the increase of load. 


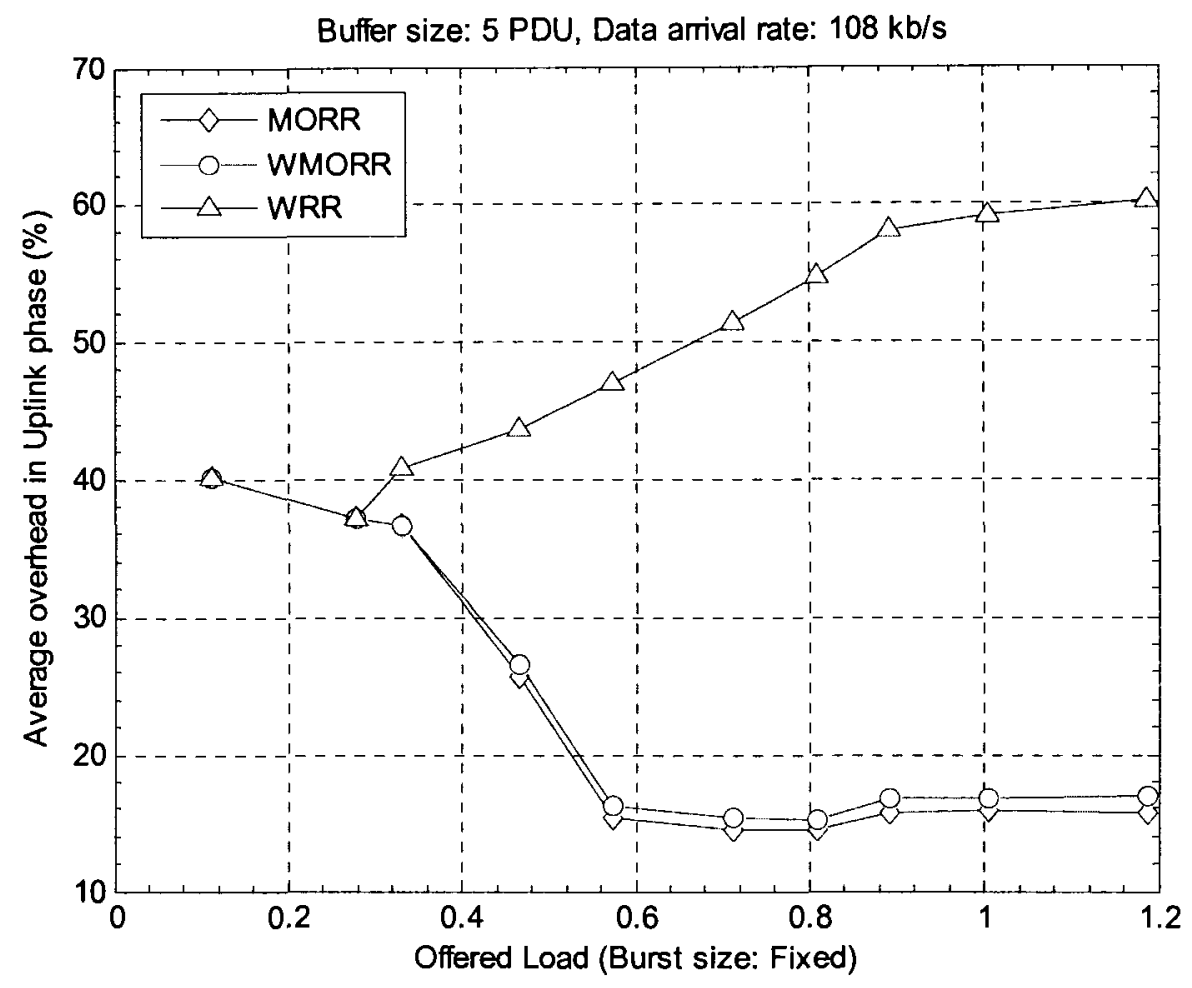

Figure 5.30. Average overhead in Uplink phase vs offered load for buffer size 5 at fixed burst.

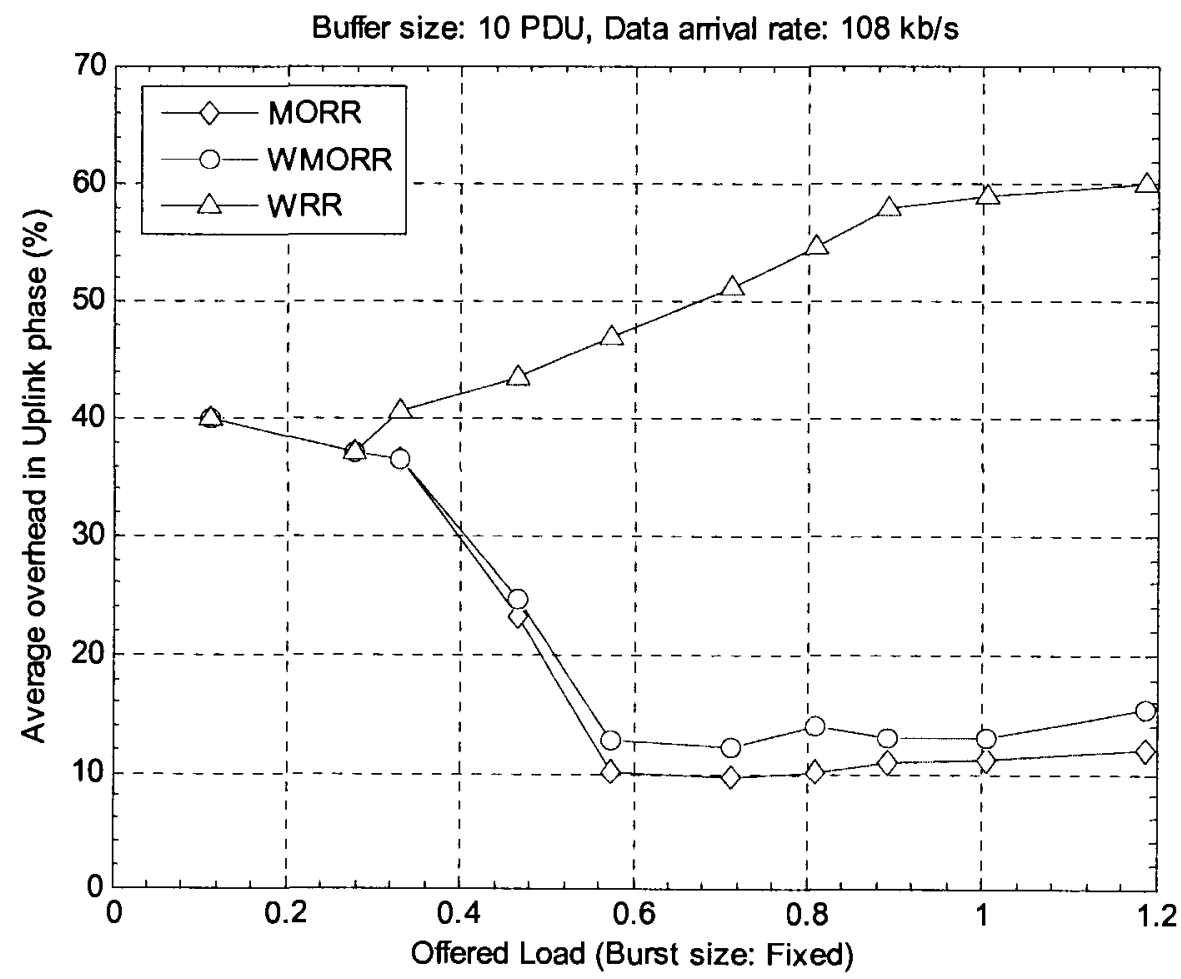

Figure 5.31. Average overhead in Uplink phase vs offered load for buffer size 10 at fixed burst. 


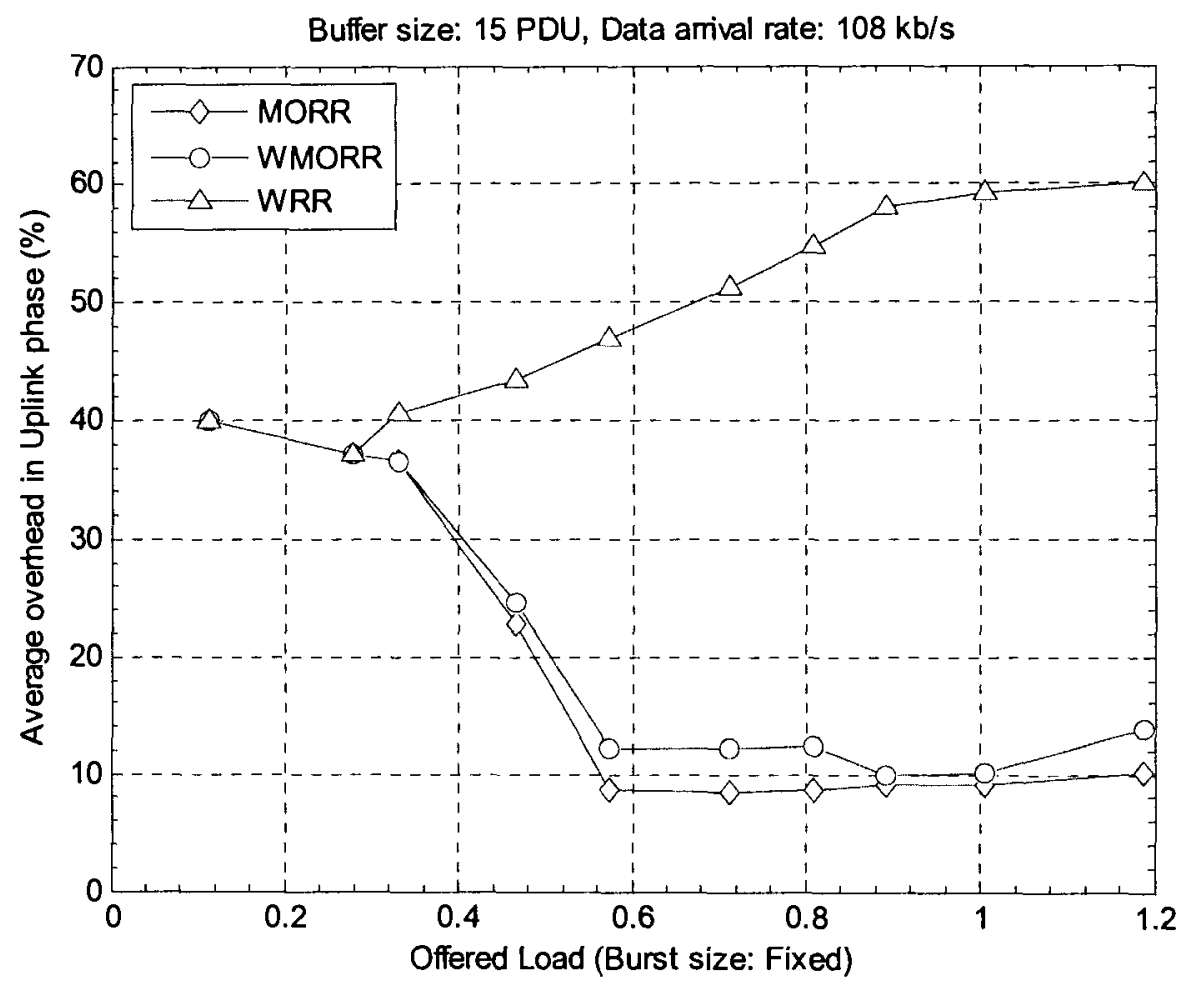

Figure 5.32. Average overhead in Uplink phase vs offered load for buffer size 15 at fixed burst.

Fig. 5.33, 5.34 and 5.35 shows the average overhead (Percentage of total uplink time) in uplink phase as a function of offered load for buffer sizes 5, 10 and 15 PDUs and the figures are for the MORR, WMORR and WRR resource allocation schemes respectively. We notice that for WMORR and MORR schemes overhead decreases with the increase of buffer size and for the WRR scheme overhead remains same for all buffer sizes. This change in overhead corresponds to the allocaion policy, as for low load all MTs are scheduled for transmission in the TDMA frame but at high load a few number of MTs are scheduled for transmission in the MORR and WMORR schemes. For the WRR scheme overhead does not depend on the buffer size as all the MTs are scheduled for transmission in the TDMA frame. 


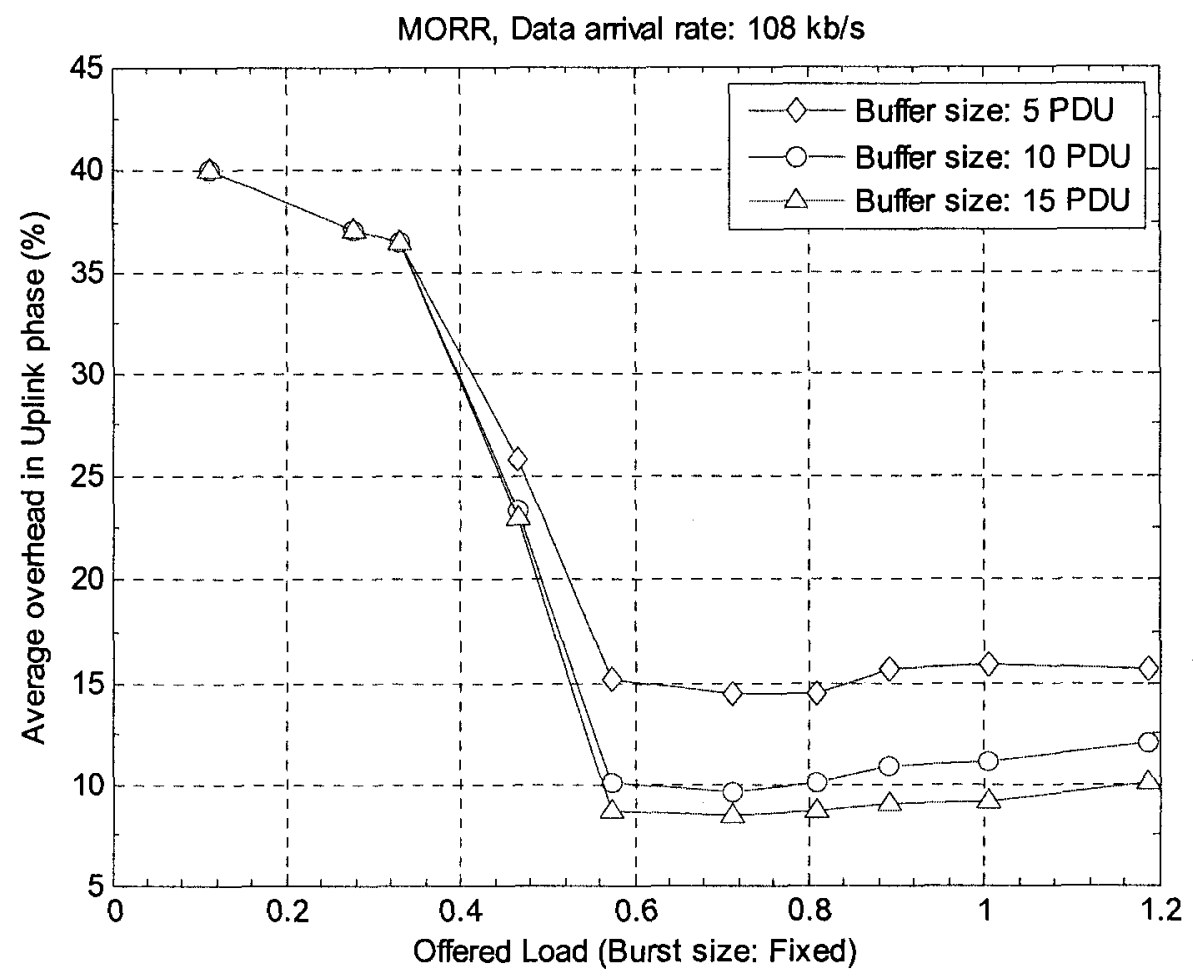

Figure 5.33. Average overhead in Uplink phase vs offered load for MORR scheme at fixed burst.

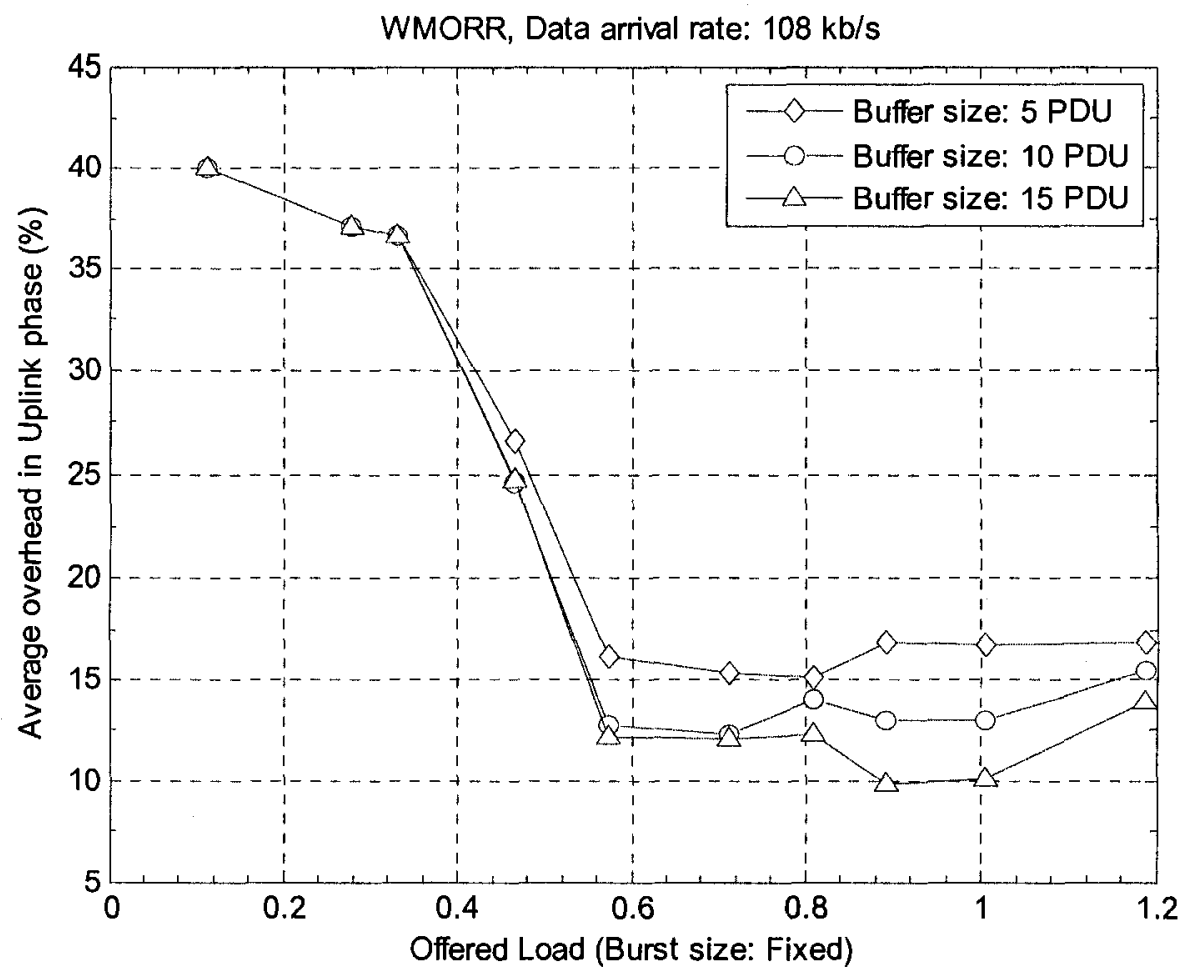

Figure 5.34. Average overhead in Uplink phase vs offered load for WMORR scheme at fixed burst. 


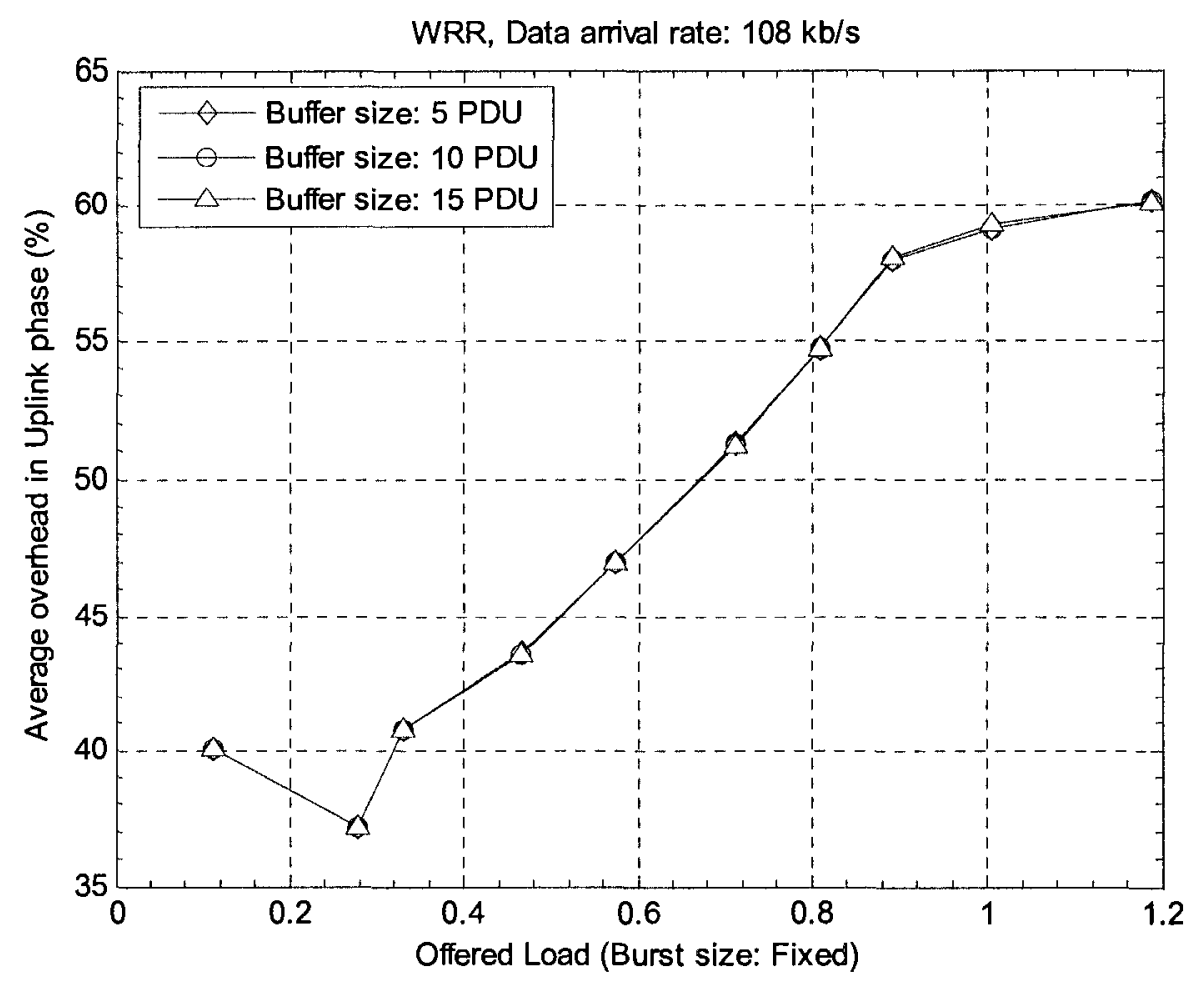

Figure 5.35. Average overhead in Uplink phase vs offered load for WRR scheme at fixed burst.

Variance in overhead of uplink phase is shown in fig. 5.36, 5.37 and 5.38 as a function of offered load for the three resource allocation schemes and the figures represent the results when the buffer size is 5,10 and 15 PDUs respectively. In the figures we noticed that the variance is symmetrical around the load value 0.5 . This is due to the reason that actual carried load below and above of this load is the same. We also notice that cahnge in variance corresponds to the curves in fig. $5.30-5.35$. 


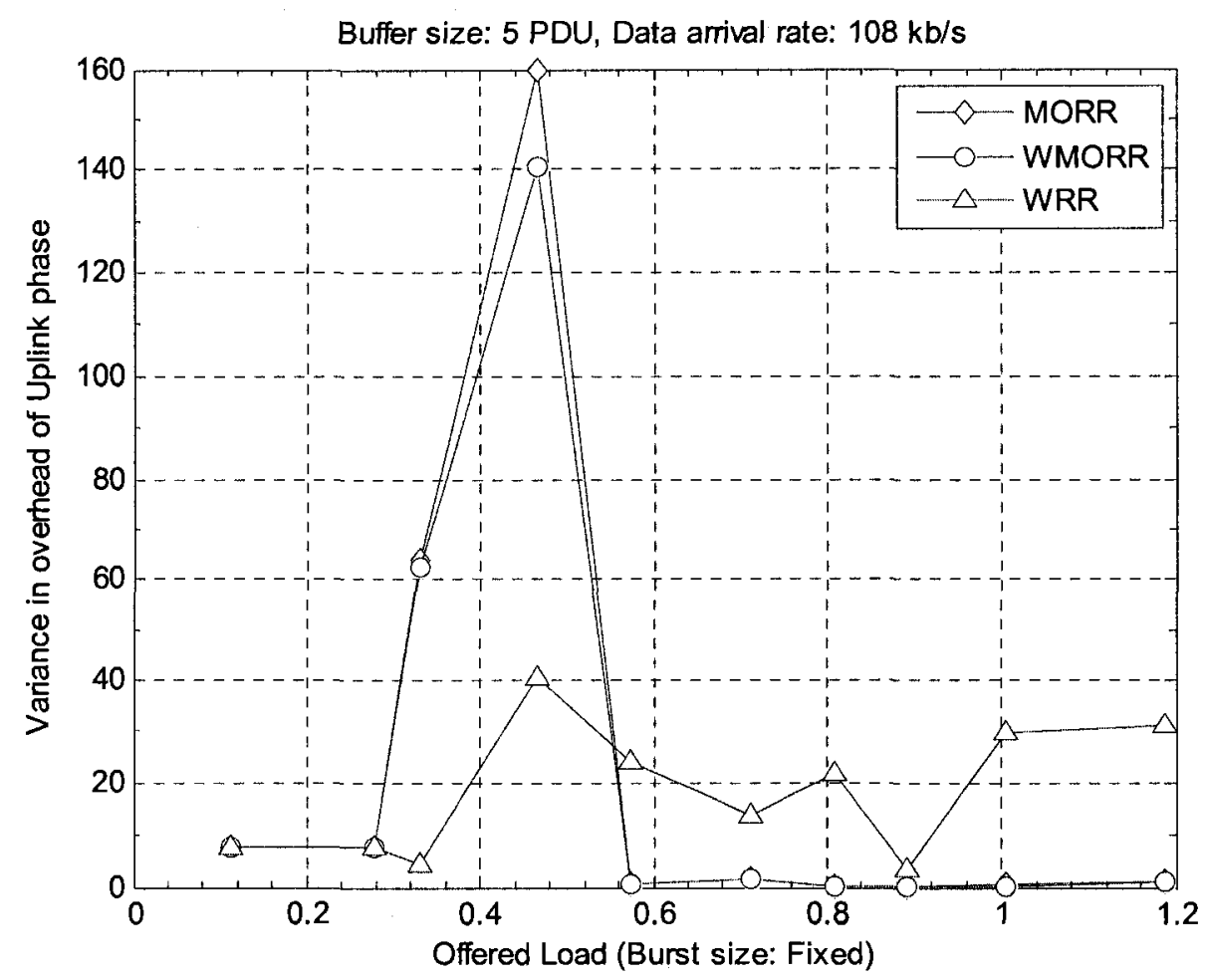

Figure 5.36. Variance in overhead of uplink phase vs offered load for buffer size 5 at fixed burst.

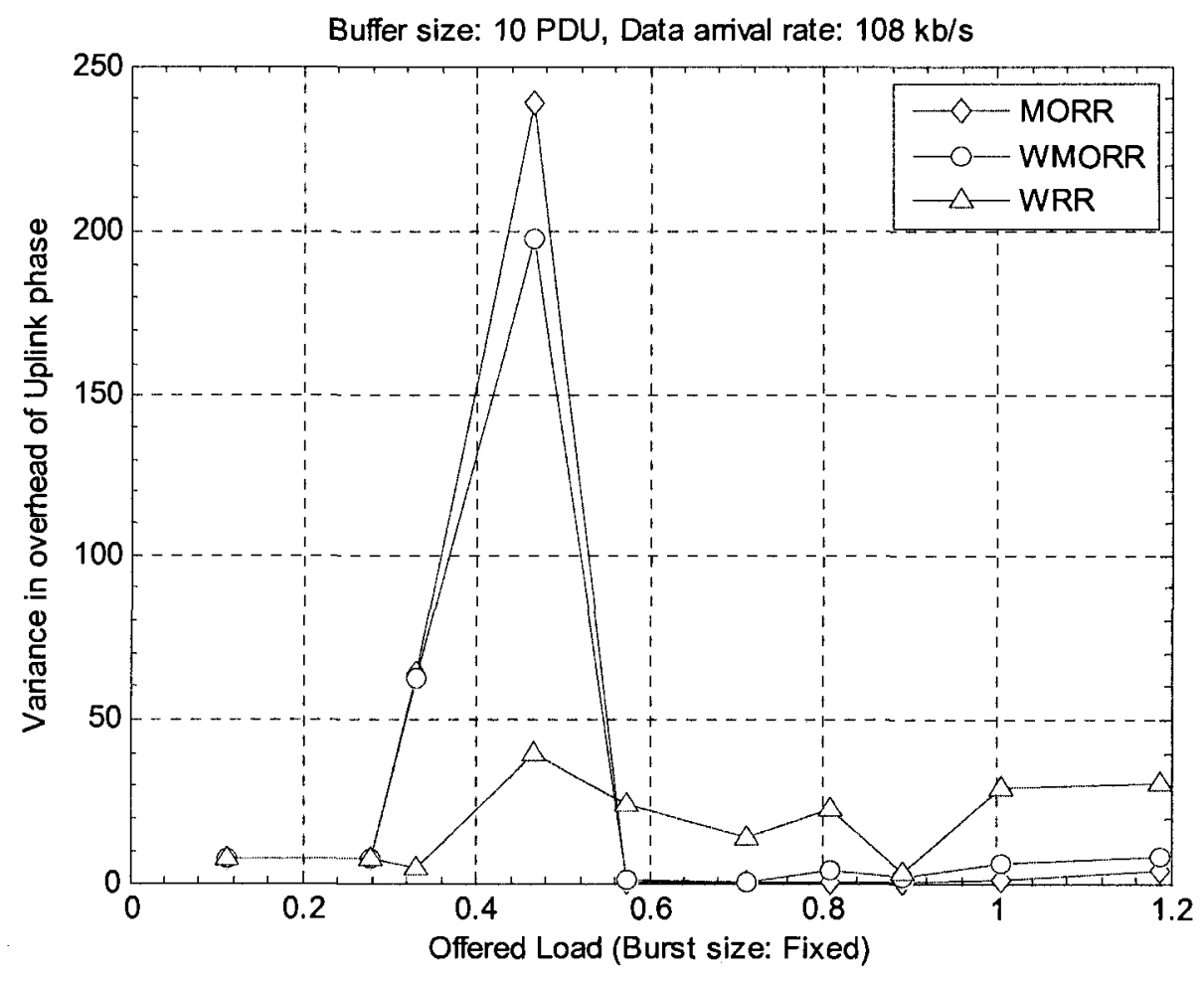

Figure 5.37. Variance in overhead of uplink phase vs offered load for buffer size 10 at fixed burst. 


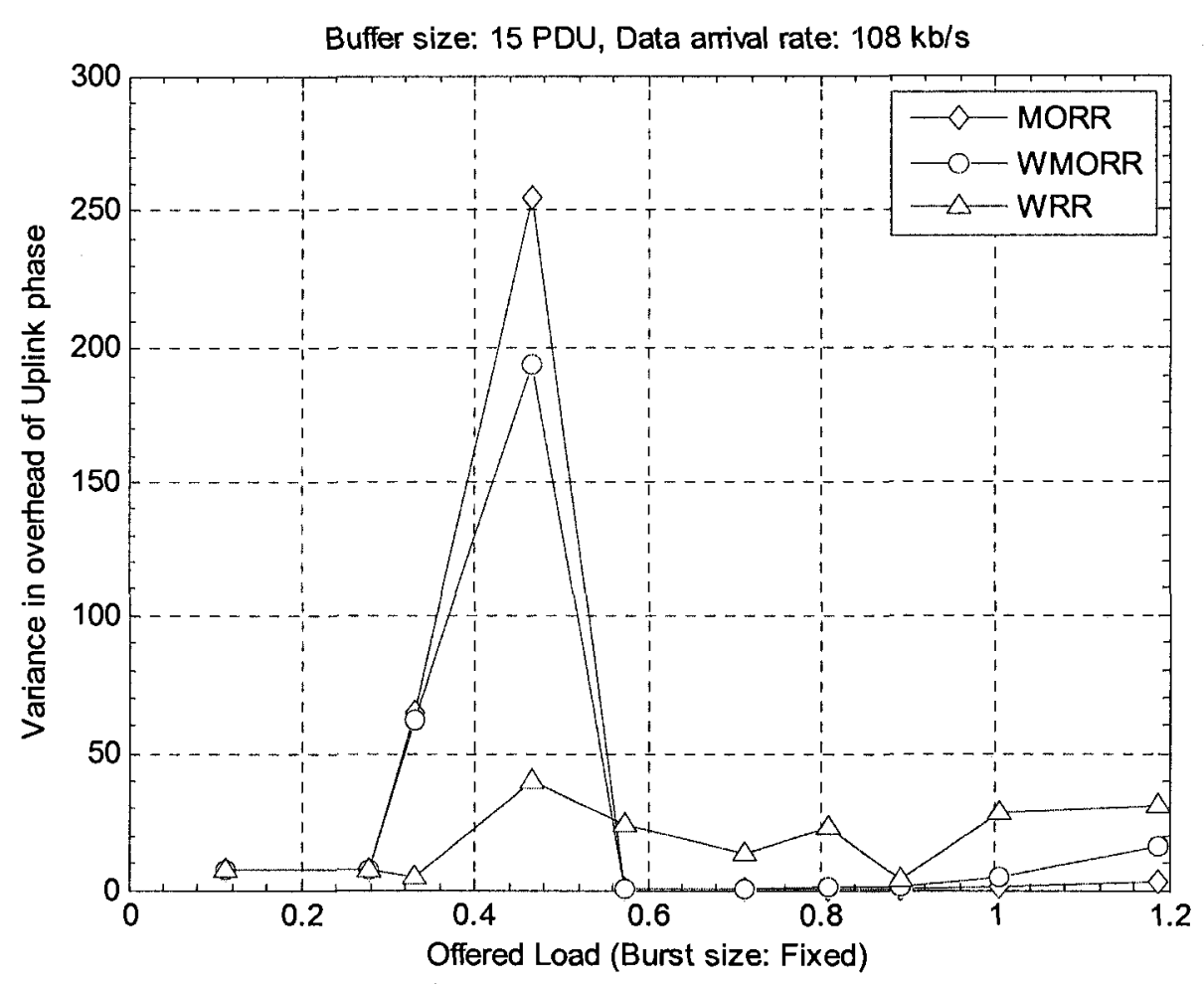

Figure 5.38. Variance in overhead of uplink phase vs offered load for buffer size 15 at fixed burst.

\section{- Average Overhead in Downlink Phase and its variance}

Fig. 5.39, 5.40 and 5.41 depict the average overhead (Percentage of downlink time) in downlink phase as a function of offered load for the three resource allocation policies, where buffer size is 5, 10 and 15 PDUs respectively. The figures show that the MORR and WMORR policies have low overhead compared to the WRR allocation scheme. For low value of offered load the downlink overhead is large as all the arrived PDU gets the transmission oppourtunity and overhead of all the user add up to give a large overhead. But as the load increases arrival rate beomes higher than the service rate and very few of the users get the transmission opportunity. This results very low overhead for MORR and WMORR scheme as when ever an user is visited, maximum capacity is assigned to it in these schemes. But for the WRR scheme as all the user buffer is visited during a TDMA frame, ovherhead of all the user adds up and the summaion continue to increase with the increase of load. 


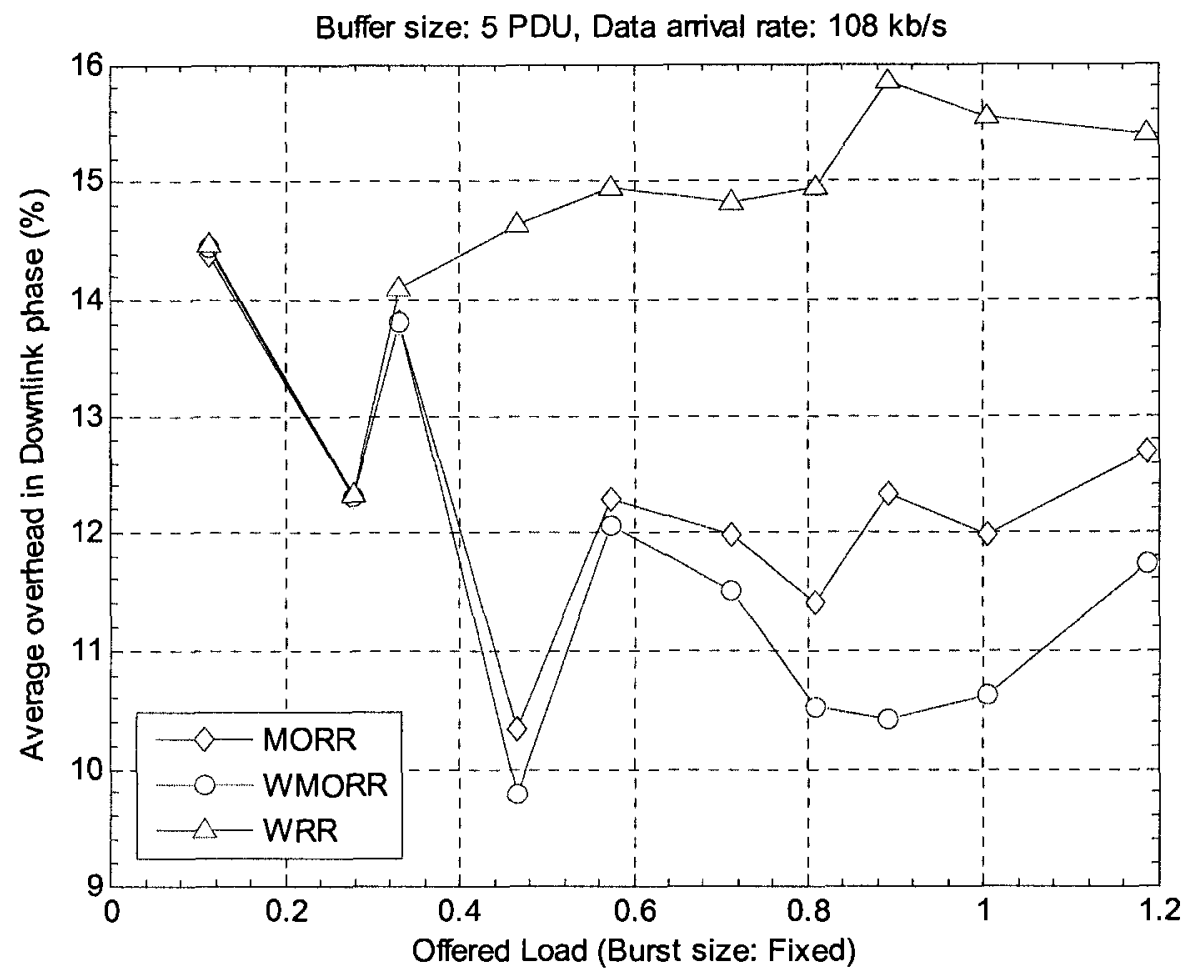

Figure 5.39. Average overhead in downlink phase vs offered load for buffer size 5 at fixed burst.

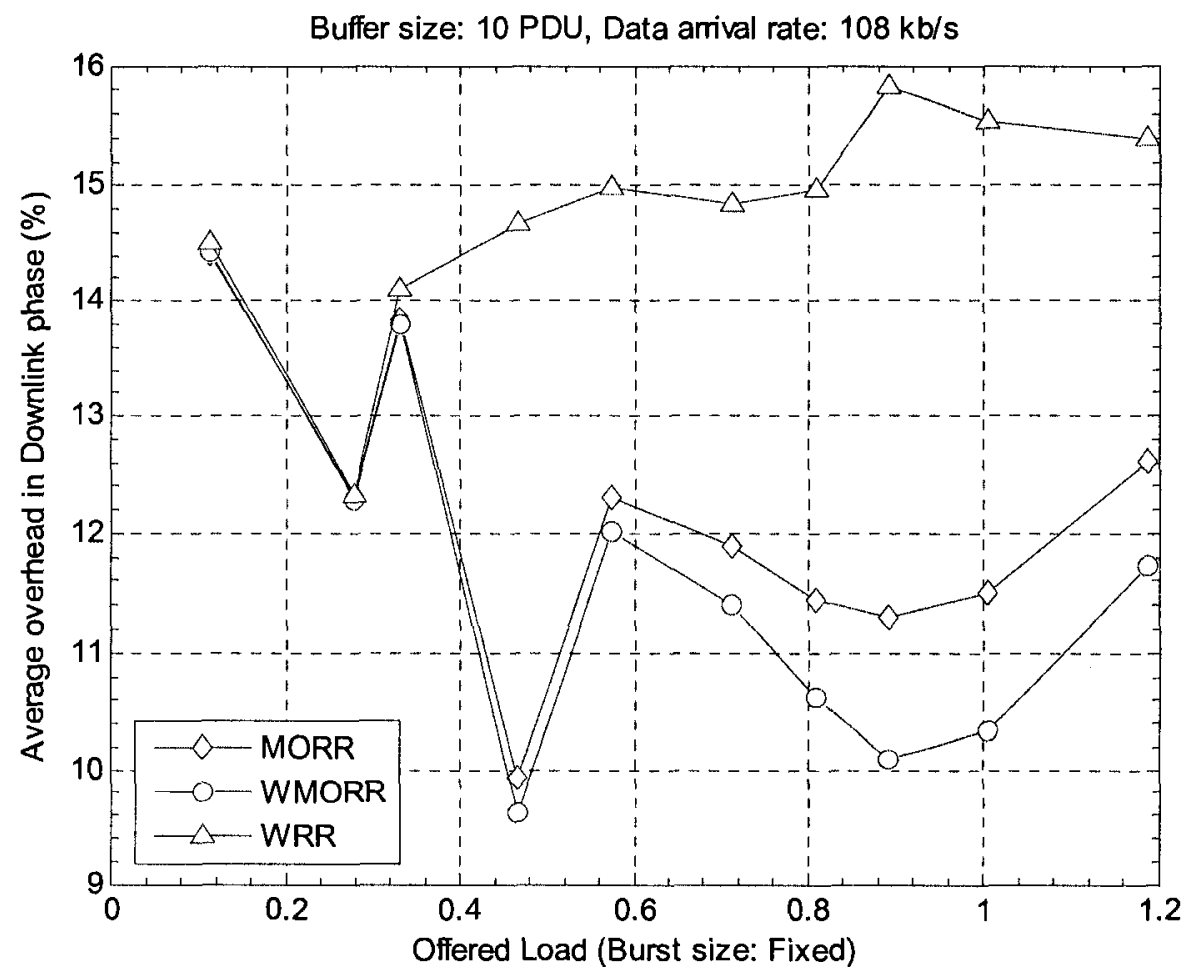

Figure 5.40. Average overhead in downlink phase vs offered load for buffer size 10 at fixed burst. 


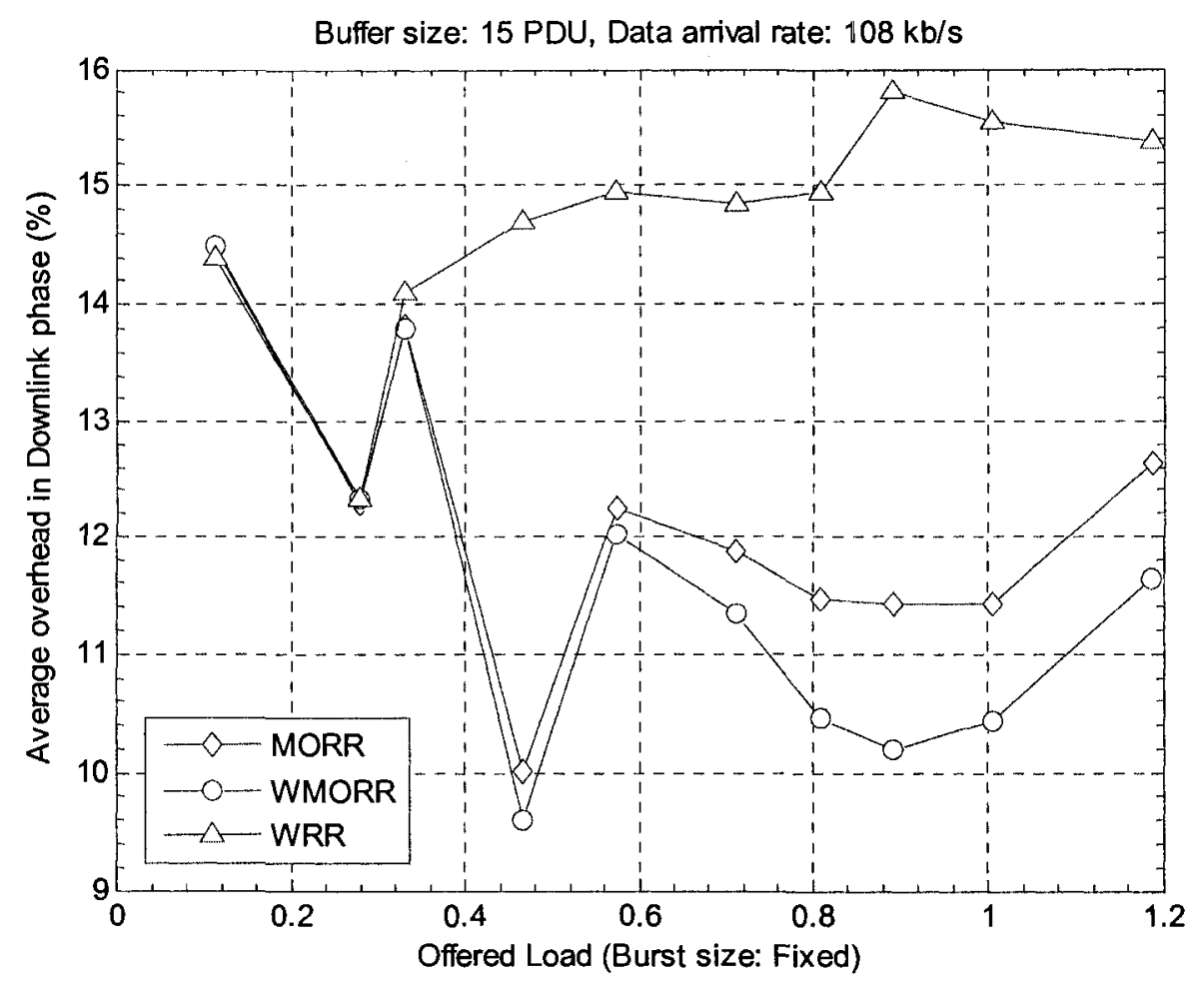

Figure 5.41. Average overhead in downlink phase vs offered load for buffer size 15 at fixed burst.

Fig. 5.42, 5.43 and 5.44 shows the average overhead in downlink phase as a function of offered load for buffer sizes 5, 10 and 15 PDUs and the figures are for the MORR, WMORR and WRR resource allocation schemes respectively. We notice that for all the schemes change in overhead does not depend on buffer size of the MT. It is due to the fact that here resource is distributed using the number of PDUs for the MTs, in the buffer of AP not using the the MT buffer size. 


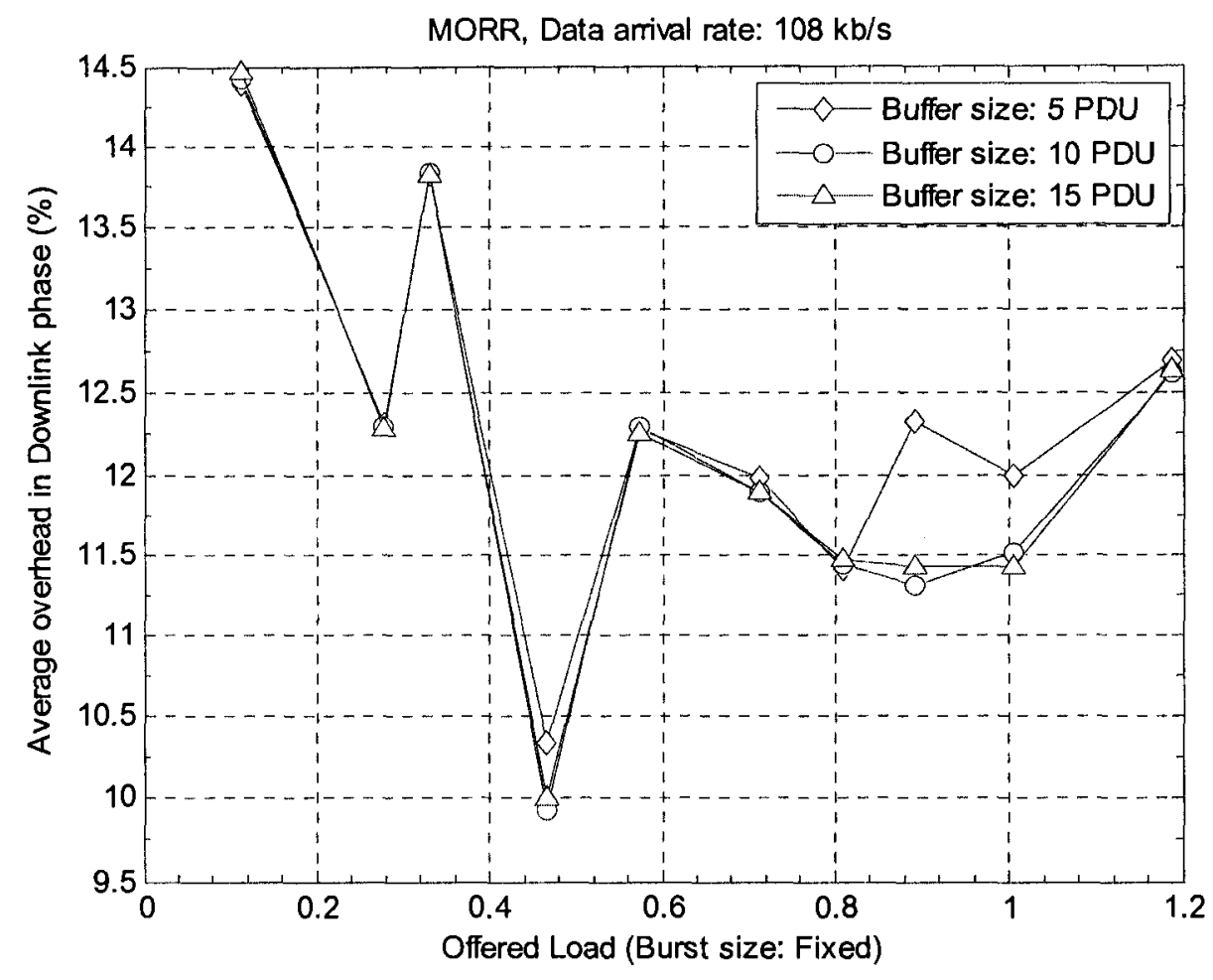

Figure 5.42. Average overhead in downlink phase vs offered load for MORR scheme at fixed burst.

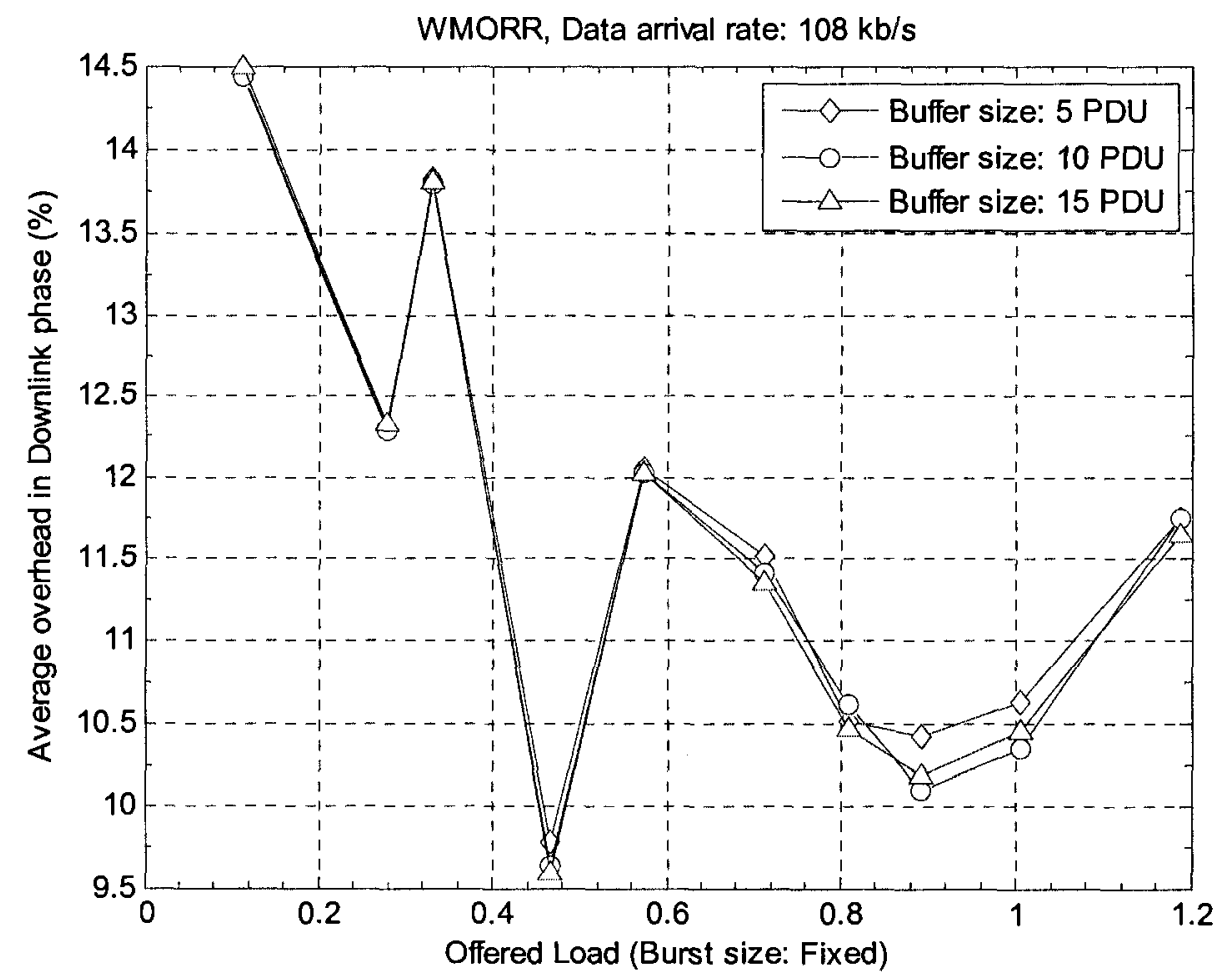

Figure 5.43. Average overhead in downlink phase vs offered load for WMORR scheme at fixed burst. 


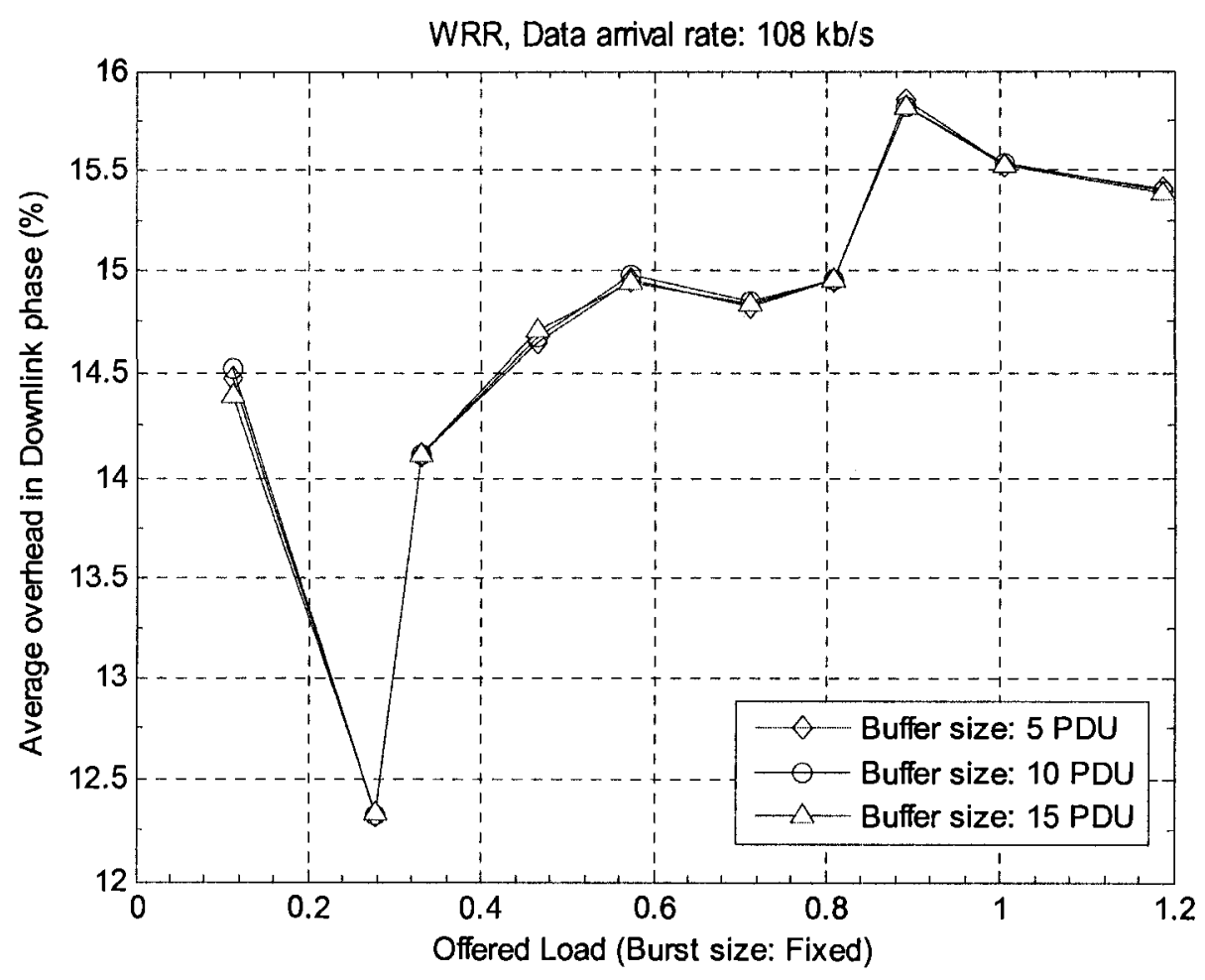

Figure 5.44. Average overhead in downlink phase vs offered load for WRR scheme at fixed burst.

Variance in overhead of downlink phase is shown in fig. 5.45, 5.46 and 5.47 as a function of offered load for the three resource allocation schemes and the figures represent the results when the buffer size is 5, 10 and 15 PDUs respectively. In the figures we noticed that change in variance corresponds to the change in curves of fig. $5.39-5.44$. 


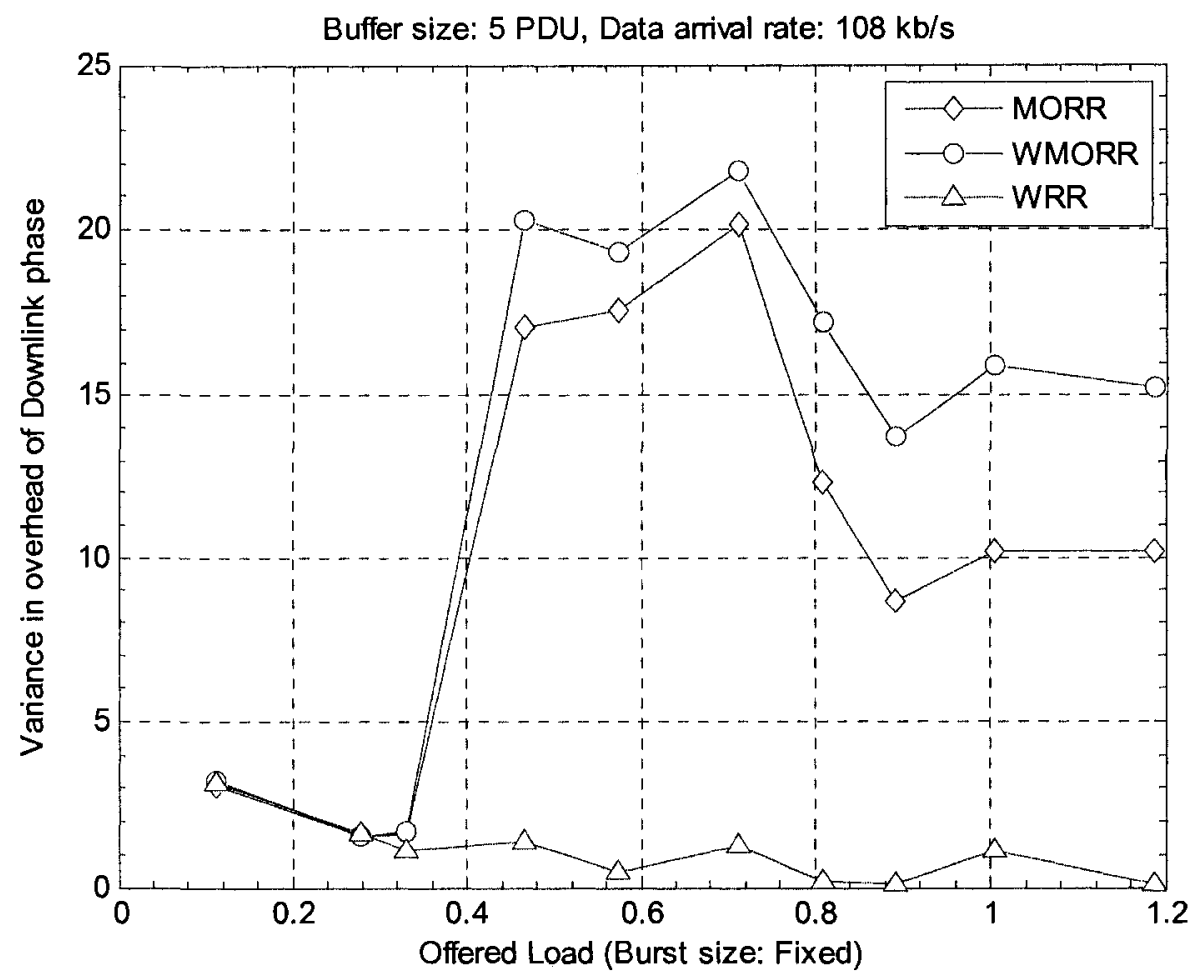

Figure 5.45. Variance in overhead of downlink phase vs offered load for buffer size 5 at fixed burst.

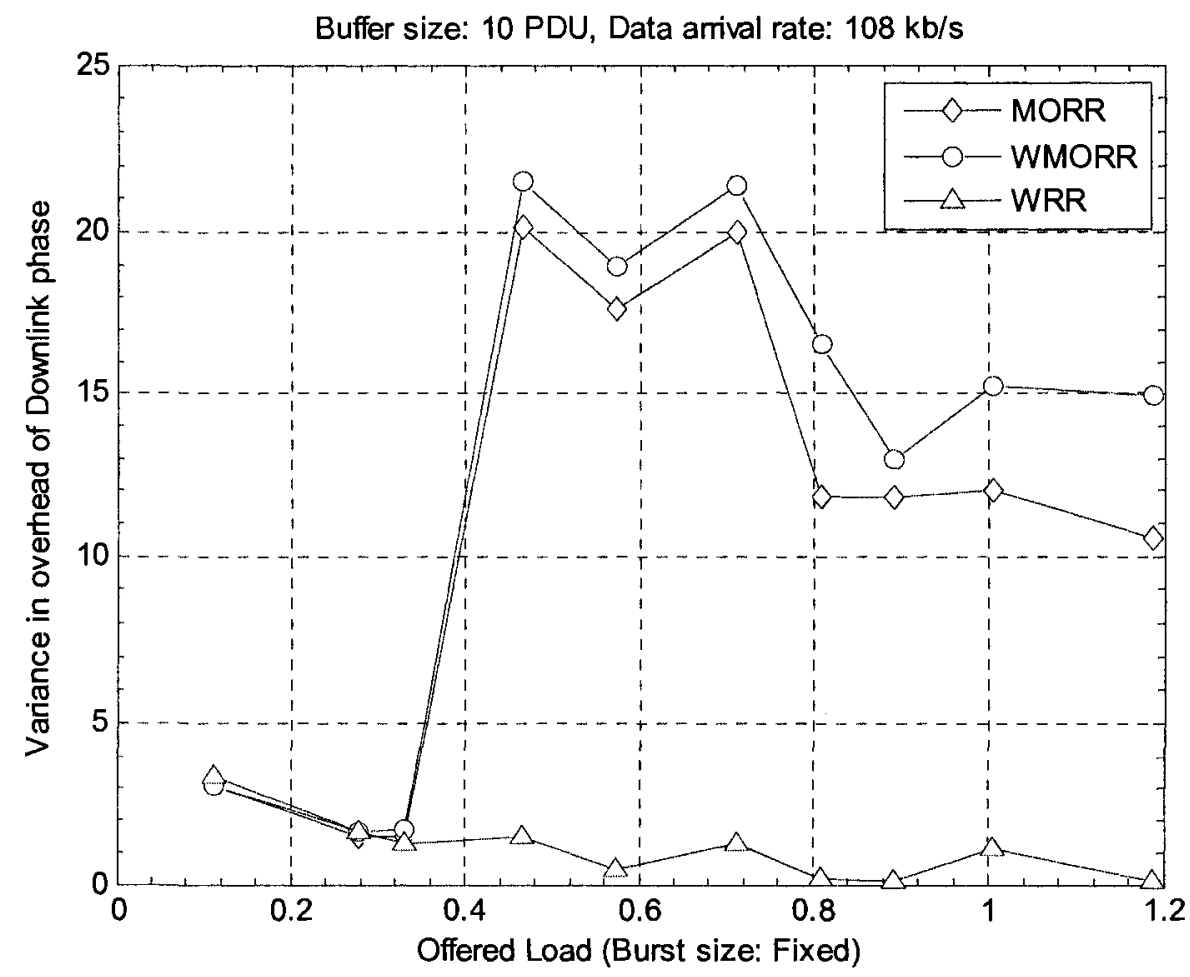

Figure 5.46. Variance in overhead of downlink phase vs offered load for buffer size 10 at fixed burst. 


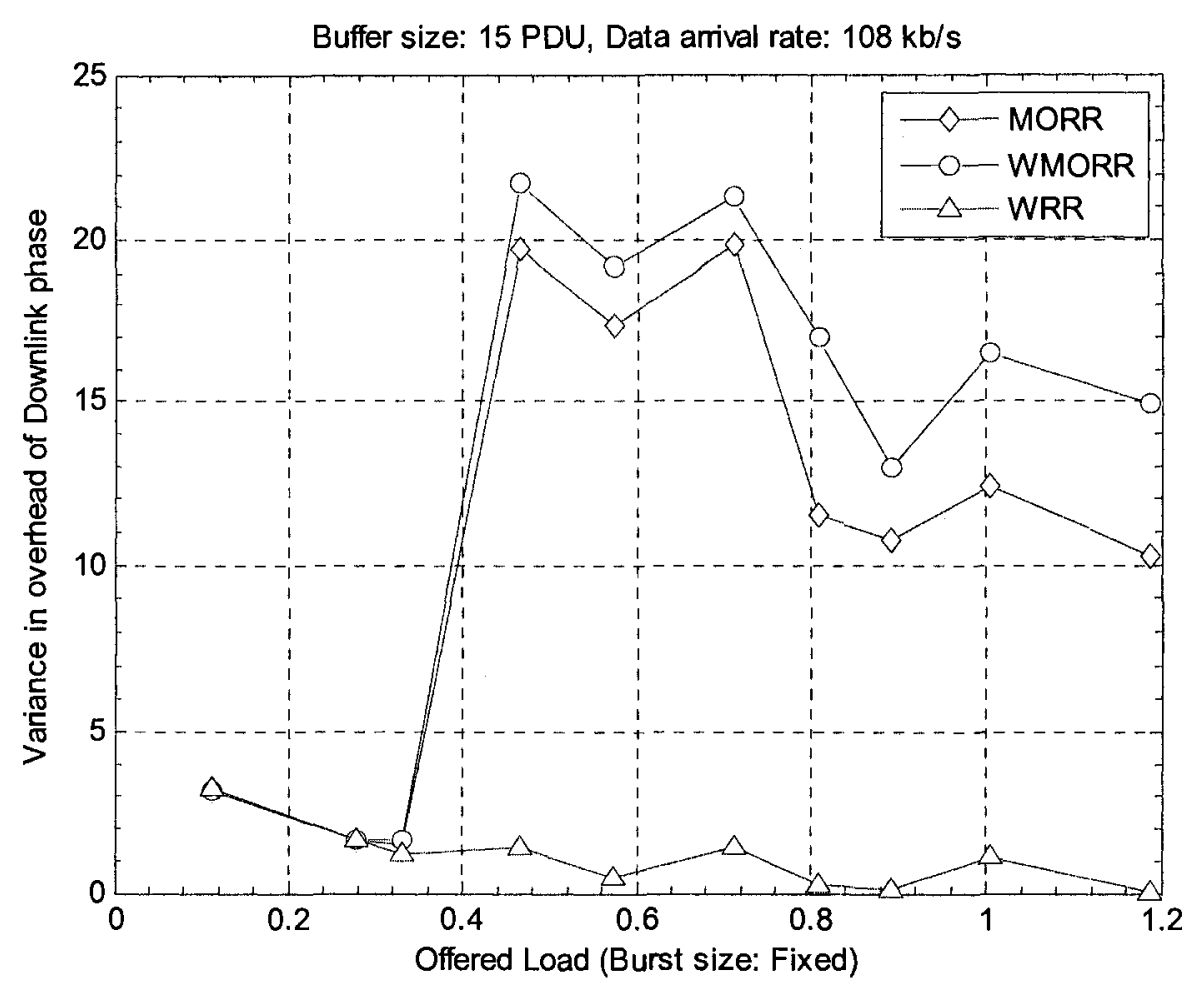

Figure 5.47. Variance in overhead of downlink phase vs offered load for buffer size 5 at fixed burst.

\subsection{Random Burst}

In the event of random burst, the simulation programme generates a random amount of data ranging from $1 \mathrm{PDU}$ to $5 \mathrm{PDU}$ per time period of $2 \mathrm{~ms}$ using the ON-OFF model (Fig. 5.1). This PDU generation rate corresponds to data arrival rate $108 \mathrm{~kb} / \mathrm{s}$ to $1024 \mathrm{~kb} / \mathrm{s}$ at the buffer of each MT. To get the number of PDUs to be generated, we call a $U(0,1)$ and multiply the outcome by 5 and rounded the result. In the on-off model we consider the probability of changing a state is equal to greater than 0.5 and length of ON or OFF time equals to one TDMA/TDD frame time or $2 \mathrm{~ms}$. We call a $\mathrm{U}(0,1)$, if the outcome is greater than or equal to 0.5 the user change its state from $O N$ to $O F F$ or vice versa. Other parameter used to setup the system is summarized in table 5.2. 
Table 5.2. SIMULATION PARAMETERS FOR RANDOM BURST.

\begin{tabular}{l|l}
\hline \multicolumn{2}{c}{ Network Parameters } \\
\hline No of CC & 1 \\
\hline No of AP & 4 \\
\hline Network area & $538 \mathrm{~m} \mathrm{x} \mathrm{538} \mathrm{m}$ \\
\hline AP coverage radius & $137.26 \mathrm{~m}$ \\
\hline AP Tx power & $1 \mathrm{Watt}$ \\
\hline Antenna height & $12 \mathrm{~m}$ \\
\hline Antennal type & Omnidirectional \\
\hline AP location & Urban area \\
\hline AP buffer size & 100 PDU \\
\hline CC buffer size & 400 PDU \\
\hline No. of RCH phase & 1 \\
\hline Simulation runtime & 10000 frames \\
\hline DL/UL time & $940 X 10^{-6} \mathrm{~s}$ \\
\hline \multicolumn{2}{c}{ Source model } \\
\hline LCH PDU length & $54 \mathrm{byte}$ \\
\hline SCH PDU length & $9 \mathrm{byte}$ \\
\hline Max. MT movement rate & $10 \mathrm{~m} / \mathrm{s}$ \\
\hline MT Buffer size & $5 / 10 / 15$ PDU \\
\hline MT Buffer Management & FIFO \\
\hline
\end{tabular}

Using the same methodology that we used for the fixed burst, we calculated the offered load for this case using the equation 5.2. In the equation the total requested time in the uplink phase of all MT associated with an $\mathrm{AP}$ is divided by the uplink capacity of that $\mathrm{AP}$ to calculate the offered load. Here the capacity of the AP is the Uplink time in a TDMA frame. Fig. 5.48 shows distribution of load over the number of mobile terminals that we get as outcome of the simulation.

$$
\begin{aligned}
\text { Load } & =\frac{\text { Total requested time per TDMA frame }}{\text { Total available time in a TDMA frame }} \\
& =\frac{\text { Total } M T \text { per AP } \times \frac{0.5 \times 2.5 \times 432}{\text { Average transmission rate per } M T}}{940 \times 10^{-6}}
\end{aligned}
$$




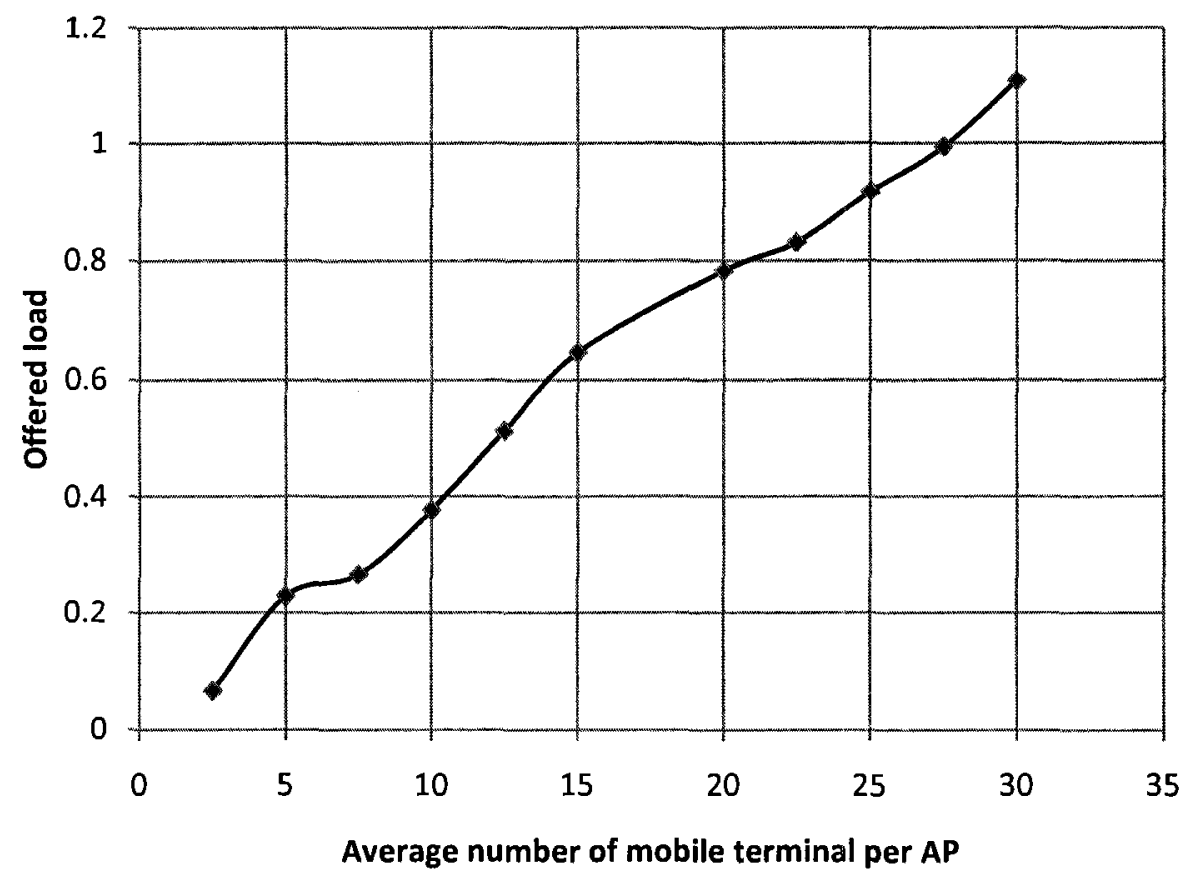

Figure 5.48. Load distribution over number of mobile terminals.

\section{- Average Number of PDUs in Buffer and its Variance}

Fig. 5.49, 5.50 and 5.51 shows the average number of PDUs in buffer of MTs as a function of offered load for the three resource allocation policies, where buffer size is 5,10 and 15 PDUs respectively. In the figures WMORR and MORR policies show almost equal performance in terms of buffer occupancy and WMORR and MORR allocation schemes also outperform the WRR scheme in terms of buffer occupancy. It is important to notice that around offered load 0.4 there is a sharp change in average number of PDUs in the user buffer for WRR scheme but the same thing happens after load value 0.65 for MORR and WMORR schemes. For the load, where the number of PDUs in the buffer is constant indicates that the amount of PDU arrival rate to the MTs is equal to the PDU service rate of MTs. Rise in number of PDUs in buffer occurs when the arrival rate becomes larger than the service rate for higher load. Our simulation system remains stable at higher load because the undelivered PDUs are stored in the buffer of MTs for future transmission. 


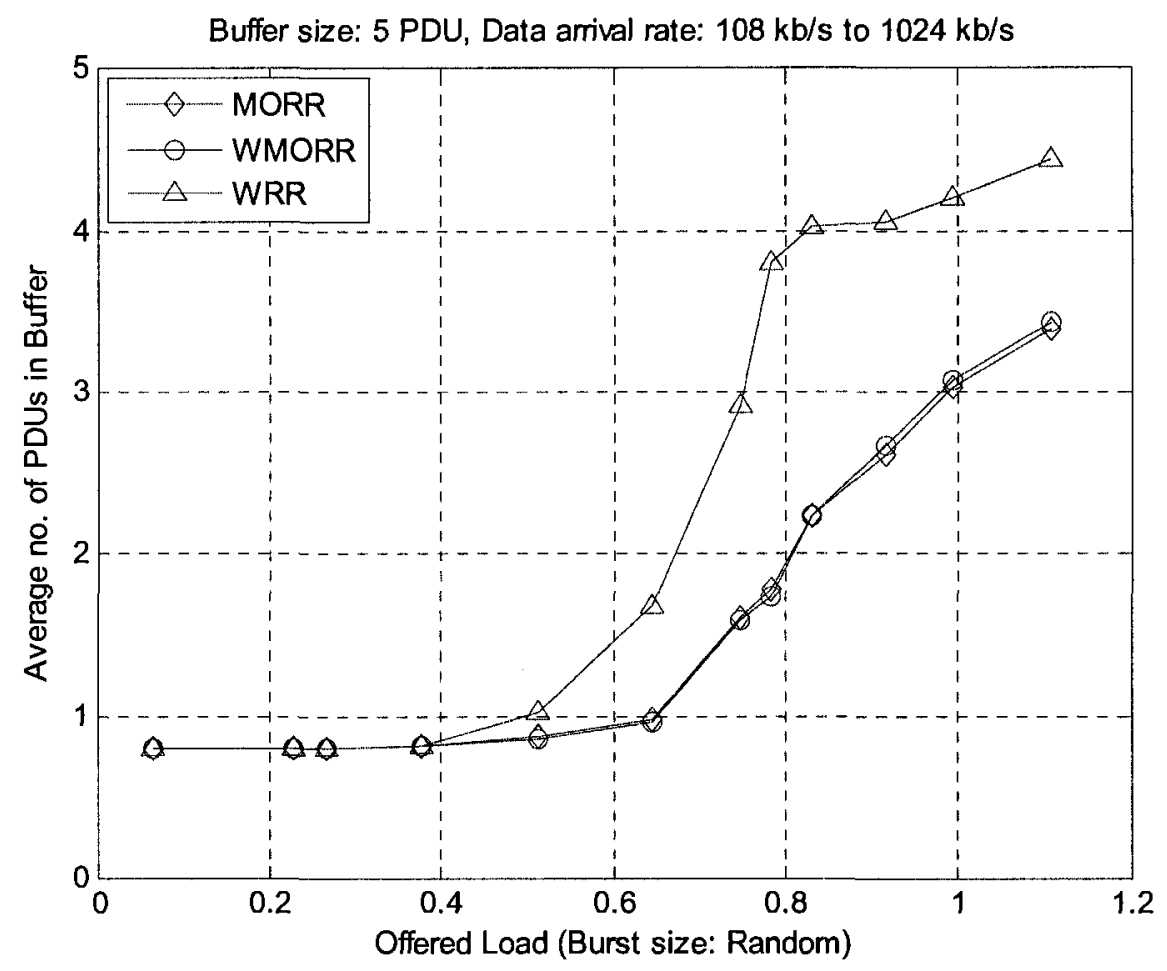

Figure 5.49. Average number of PDUs in buffer vs offered load for buffer size 5 at random burst.

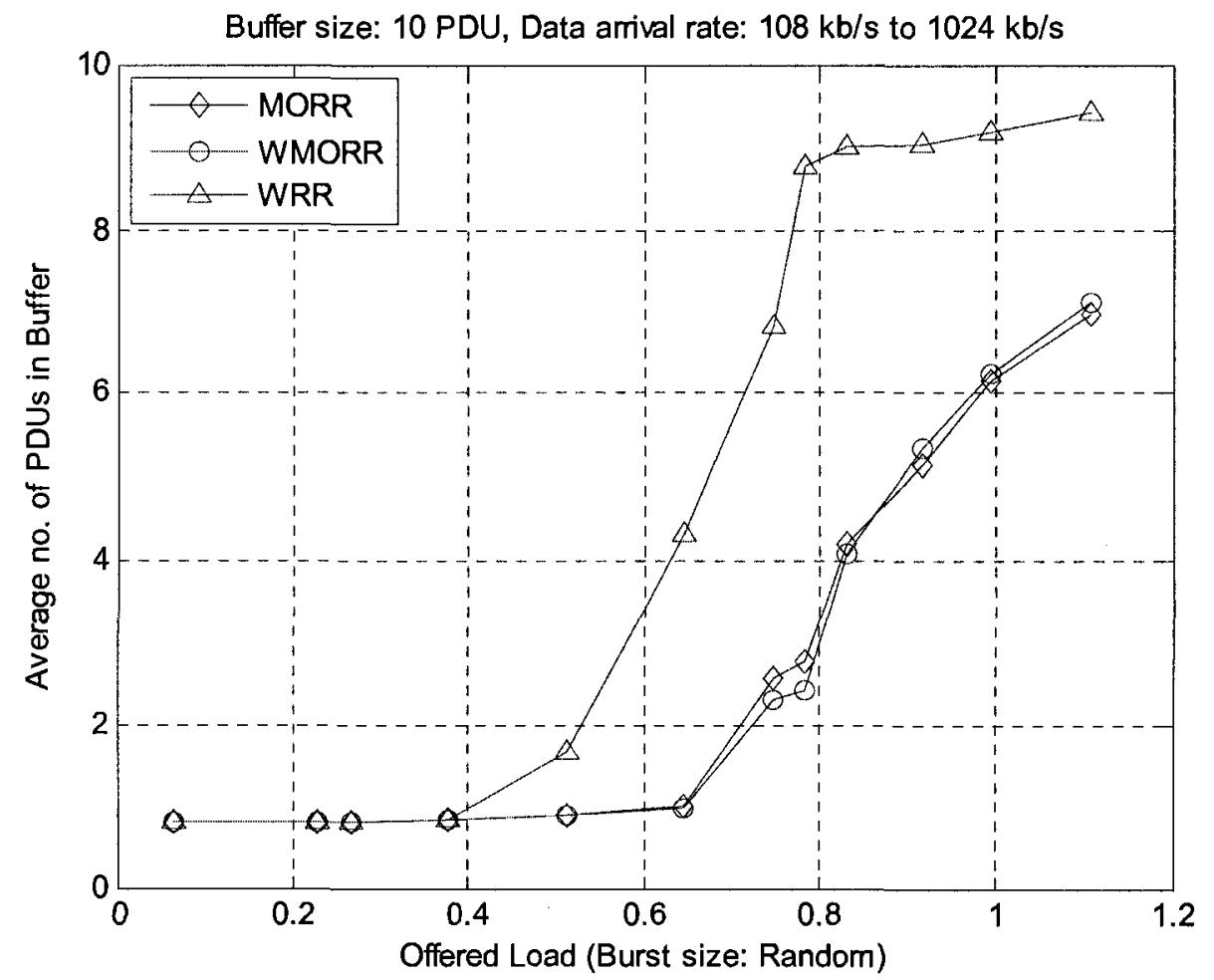

Figure 5.50. Average number of PDUs in buffer vs offered load for buffer size 10 at random burst. 


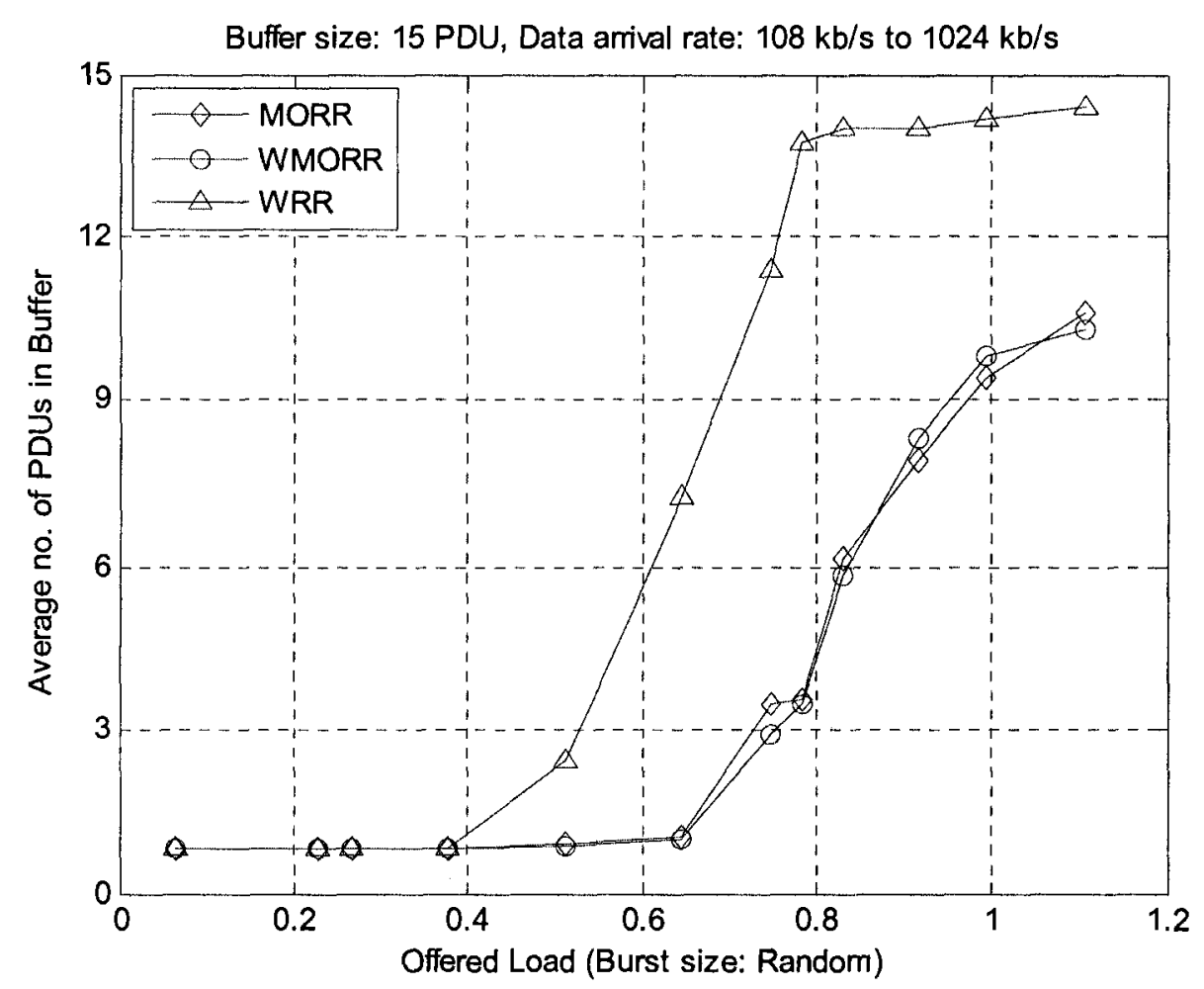

Figure 5.51. Average number of PDUs in buffer vs offered load for buffer size 15 at random burst.

Fig. 5.52, 5.53 and 5.54 show the average number of PDUs in buffer of MTs as a function of offered load for the buffer size 5, 10 and 15 PDUs and the figures are for the MORR, WMORR and WRR resource allocation schemes respectively. The figures show that at very low offered load buffer occupancy does not depend on the size of buffer and as the load increasess number of PDUs in the buffer also increases. For the WRR scheme average number of PDUs in the buffer of MTs becomes almost constant and equal to the buffer size at loads higher than 0.8. On the other hand for MORR and WMORR scehmes buffers do not reach at satureation around this high load. 


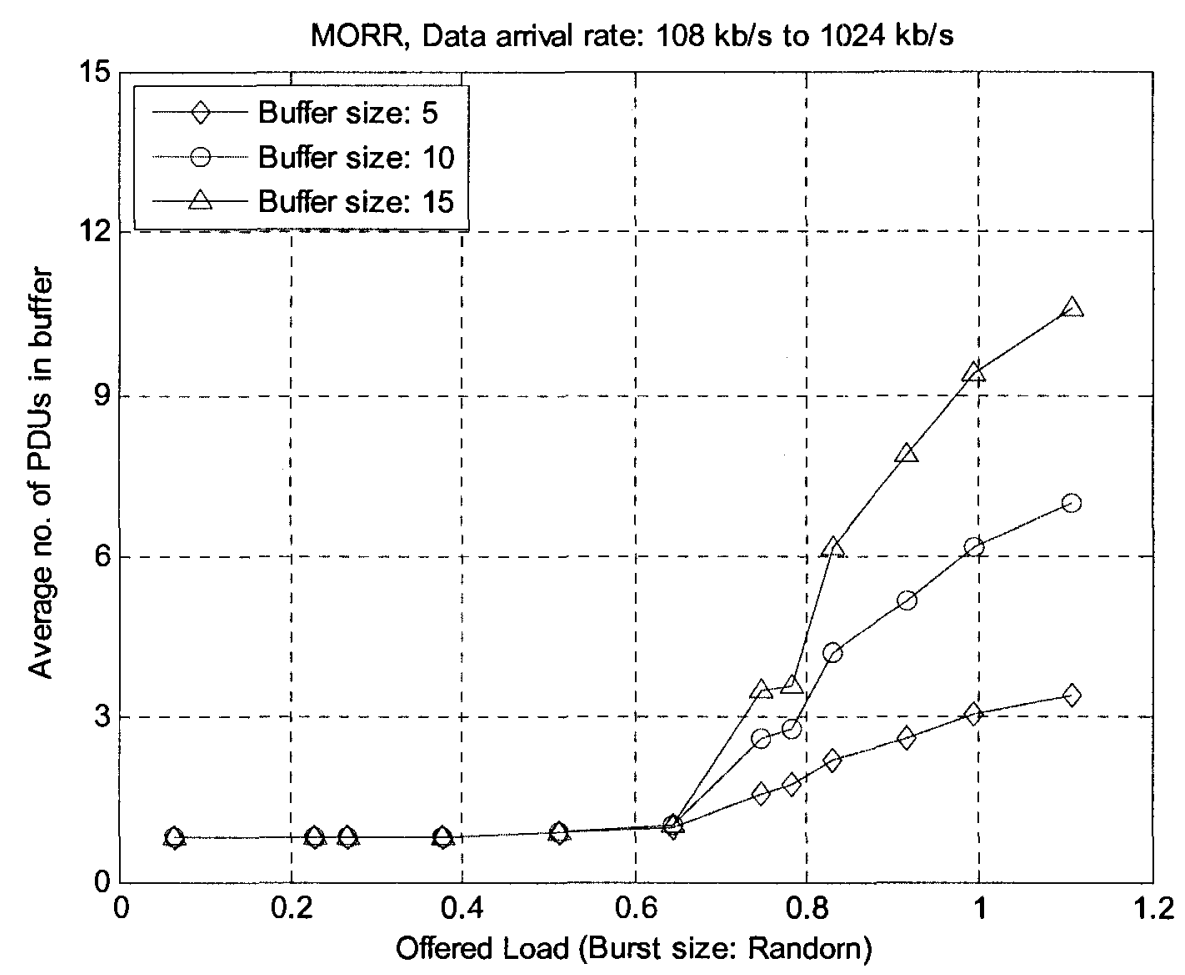

Figure 5.52. Average number of PDUs in buffer vs offered load for MORR scheme at random burst.

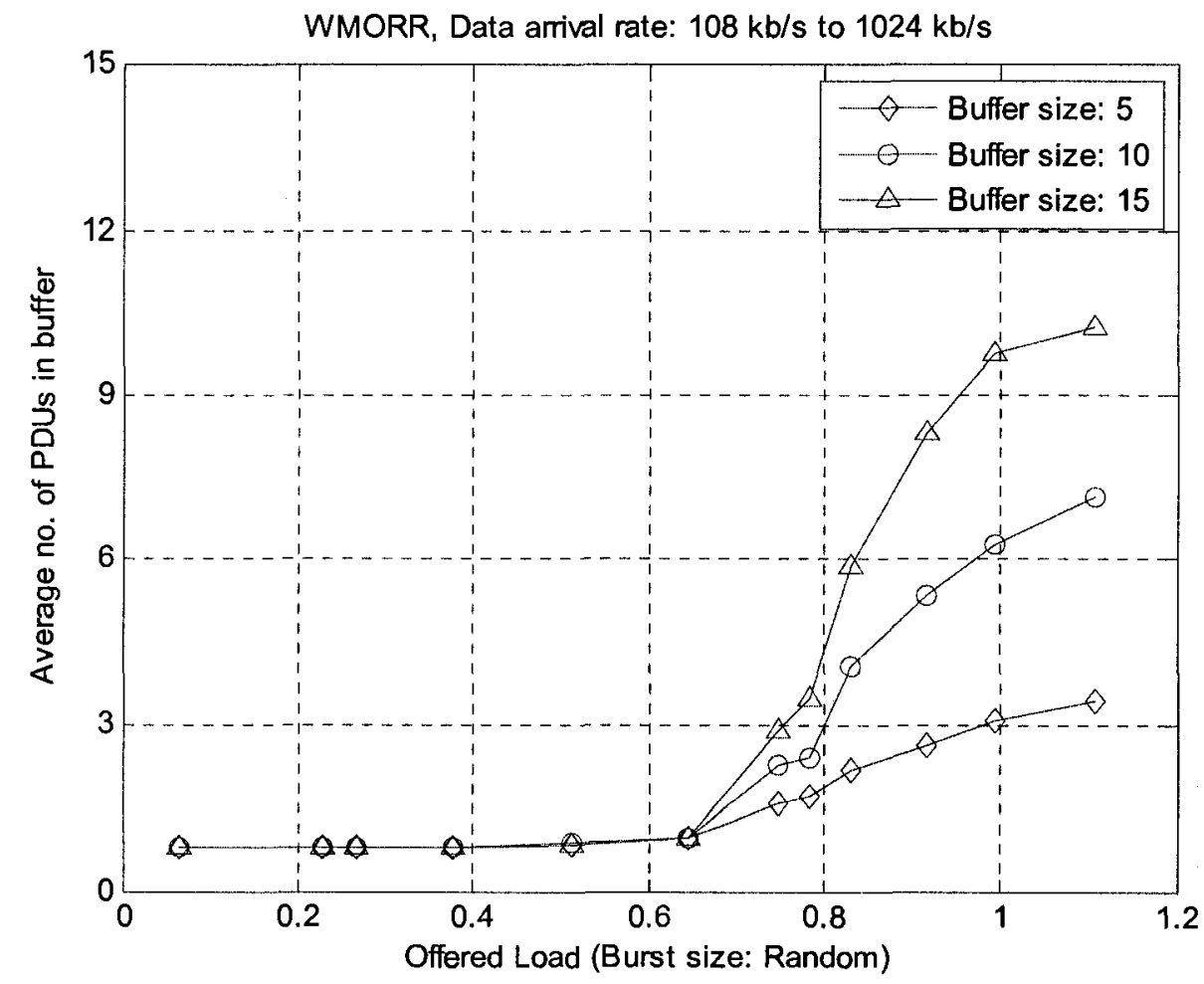

Figure 5.53. Average number of PDUs in buffer vs offered load for WMORR at random burst. 


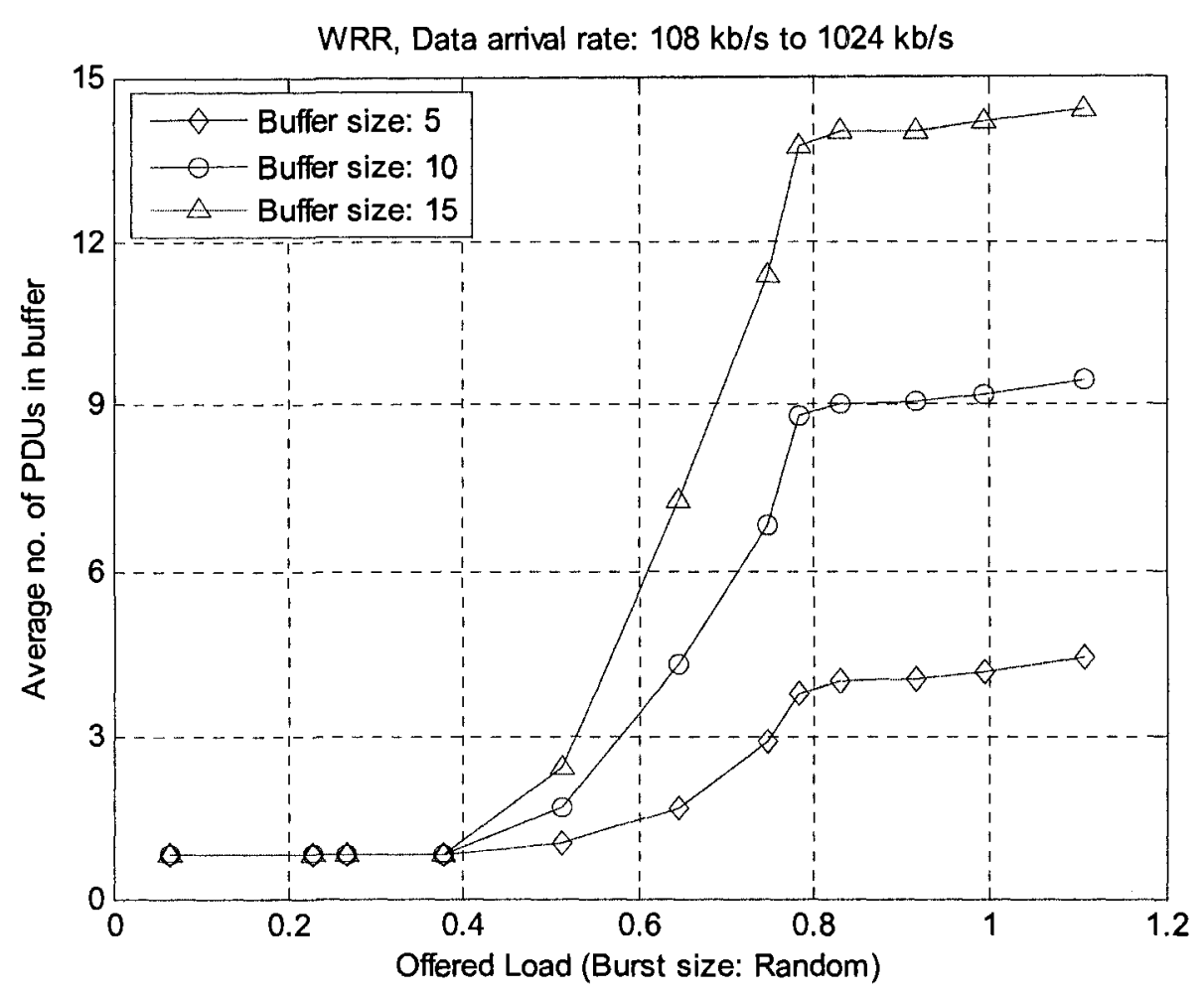

Figure 5.54. Average number of PDUs in buffer vs offered load for WRR at random burst.

Variance in the number of PDUs in the buffer of MTs is shown in the fig. 5.55, 5.56 and 5.57 as a function of offered load for the three resource allocation schemes and the figures represent the curves when the buffer size is 5,10 and 15 PDUs respectively. It is important to notice that the change in variance in the following curves corresponsds to the fig. 5.49-5.54. For the WRR scheme variance is large when load is in the range 0.4 to 0.8 and for the MORR and WMORR variance is large when the load is beyond 0.8 . We also notice that for the WRR scheme variance curve is symmetrical around the load value 0.6 and for MORR and WMORR this happens around load value 0.8 . This is due to the actual carried load below and above of these load value is same. This system behaviour indicates that for the WRR schem system is operatable below load value 0.6 on the other hand for MORR and WMORR it is 0.8 . 


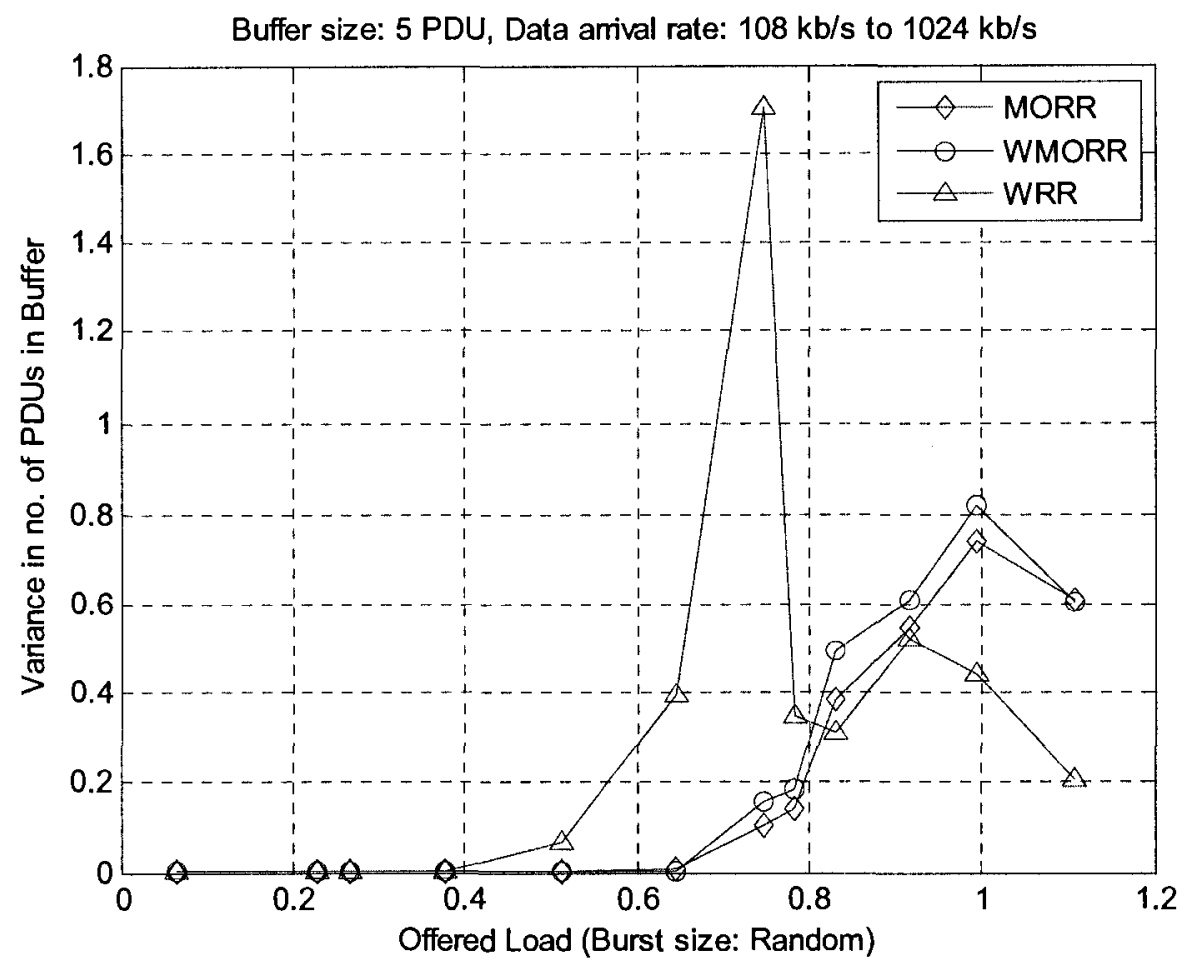

Figure 5.55. Variance in number of PDUs in buffer vs offered load for buffer size 5 at random burst.

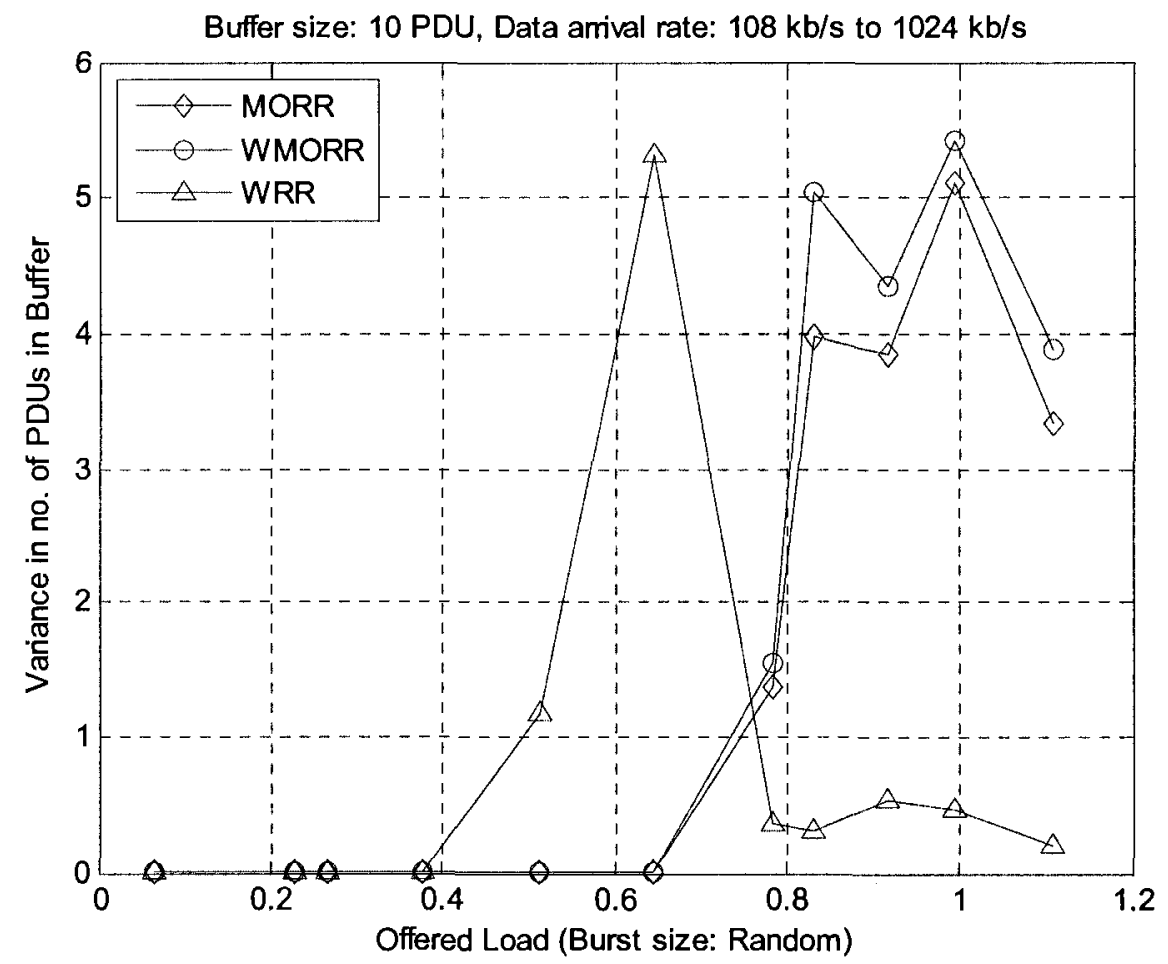

Figure 5.56. Variance in number of PDUs in buffer vs offered load for buffer size 10 at random burst. 


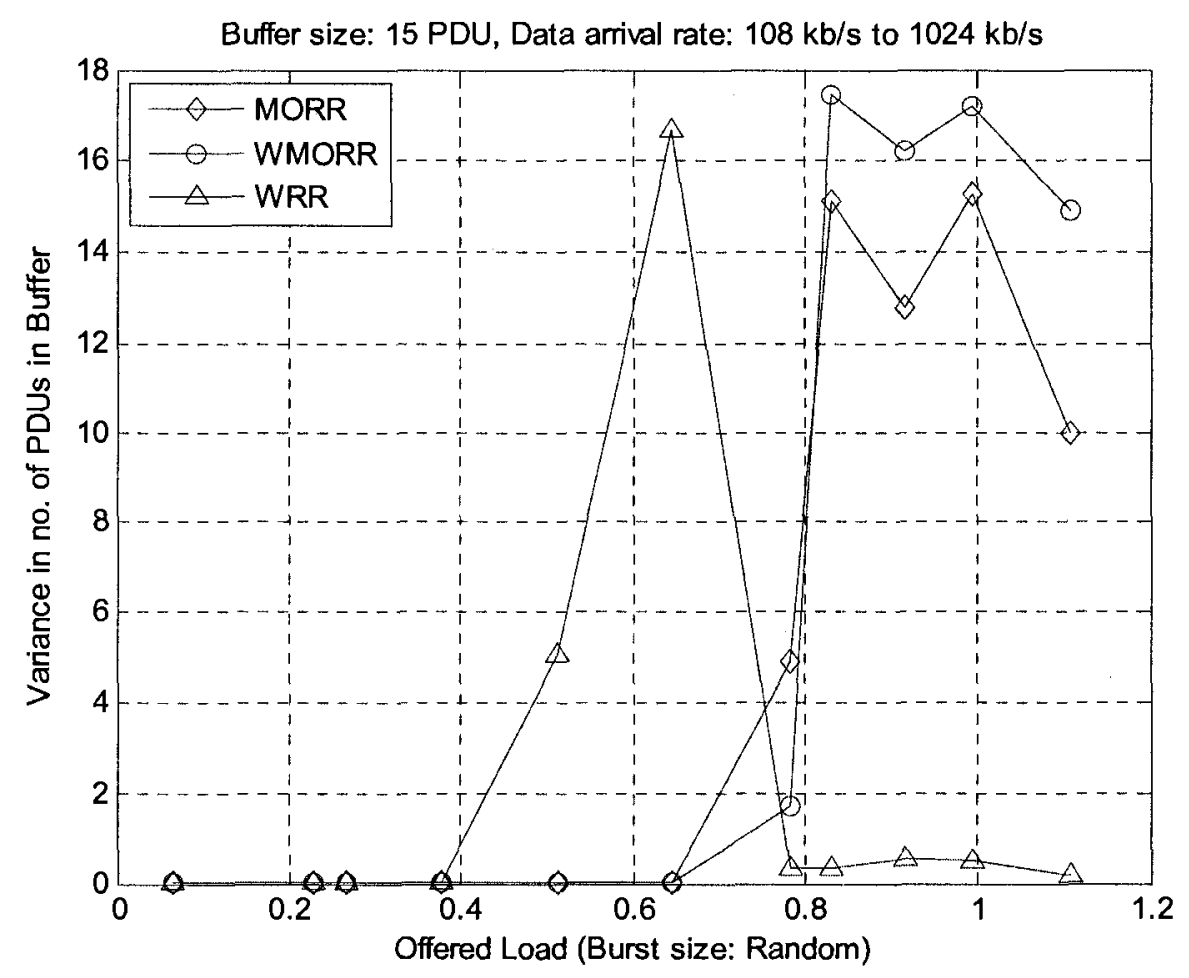

Figure 5.57. Variance in number of PDUs in buffer vs offered load for buffer size 15 at random burst.

\section{- Probability of Buffer Overflow and its variance}

Figs. 5.58, 5.59 and 5.60 depict the probability of buffer overflow as a function of offered load for the three resource allocation policies, where buffer size is 5, 10 and 15 PDUs respectively. The figures show that the WMORR allocation scheme has the lowest probability of overflow among the three schemes. It is important to note that probability of overflow is zero when the load is very low and it starts to increase when the offered load is increased above 0.5 for WRR and 0.6 for MORR and WMORR schemes. These value of load is significant because at load higher than this the total PDU arrival rate at our system becomes higher than the PDU service rate and the numbers of PDUs in the buffer starts to increase (Fig. 5.49 to 5.54 ) . 


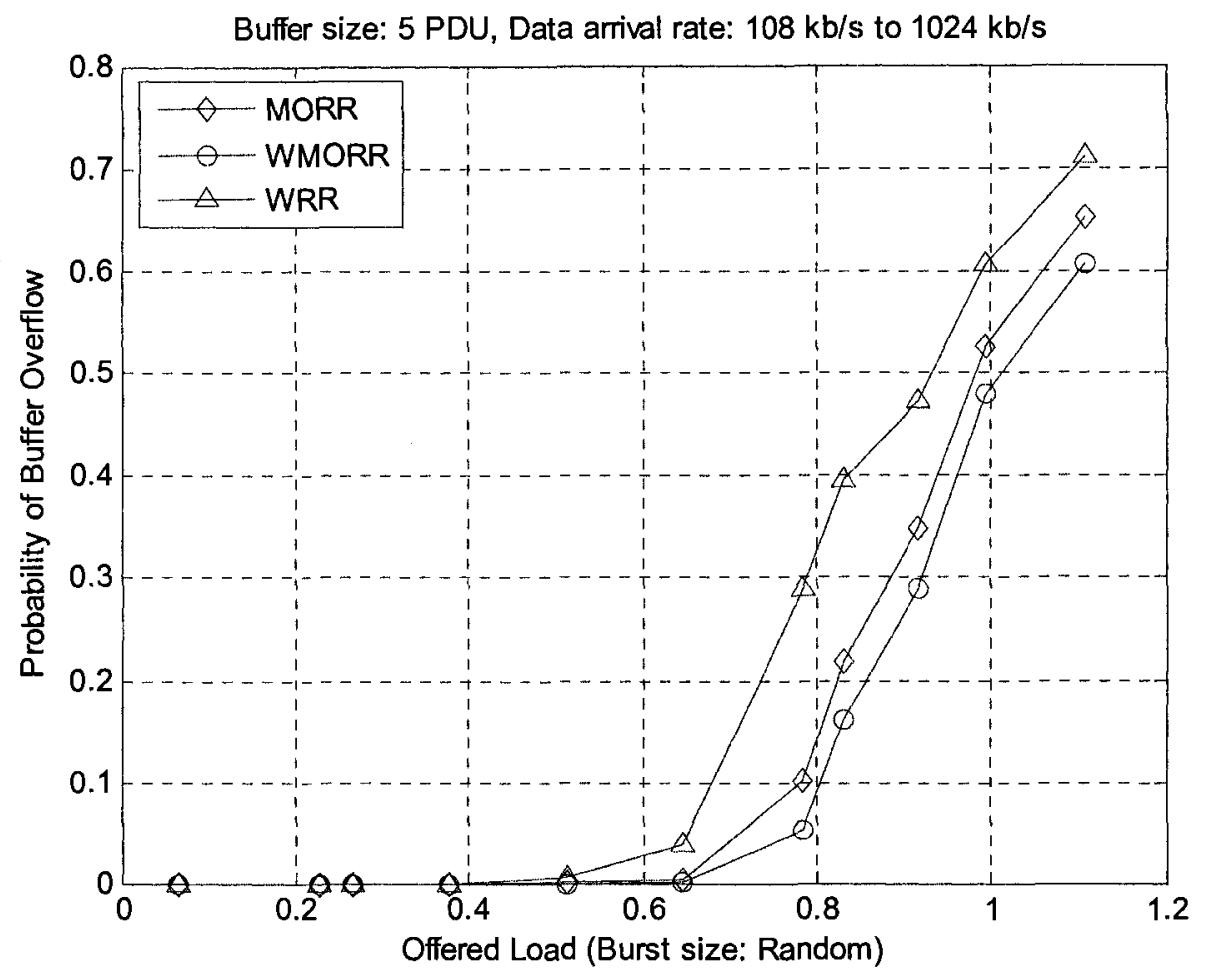

Figure 5.58. Probability of buffer overflow vs offered load for buffer size 5 at random burst.

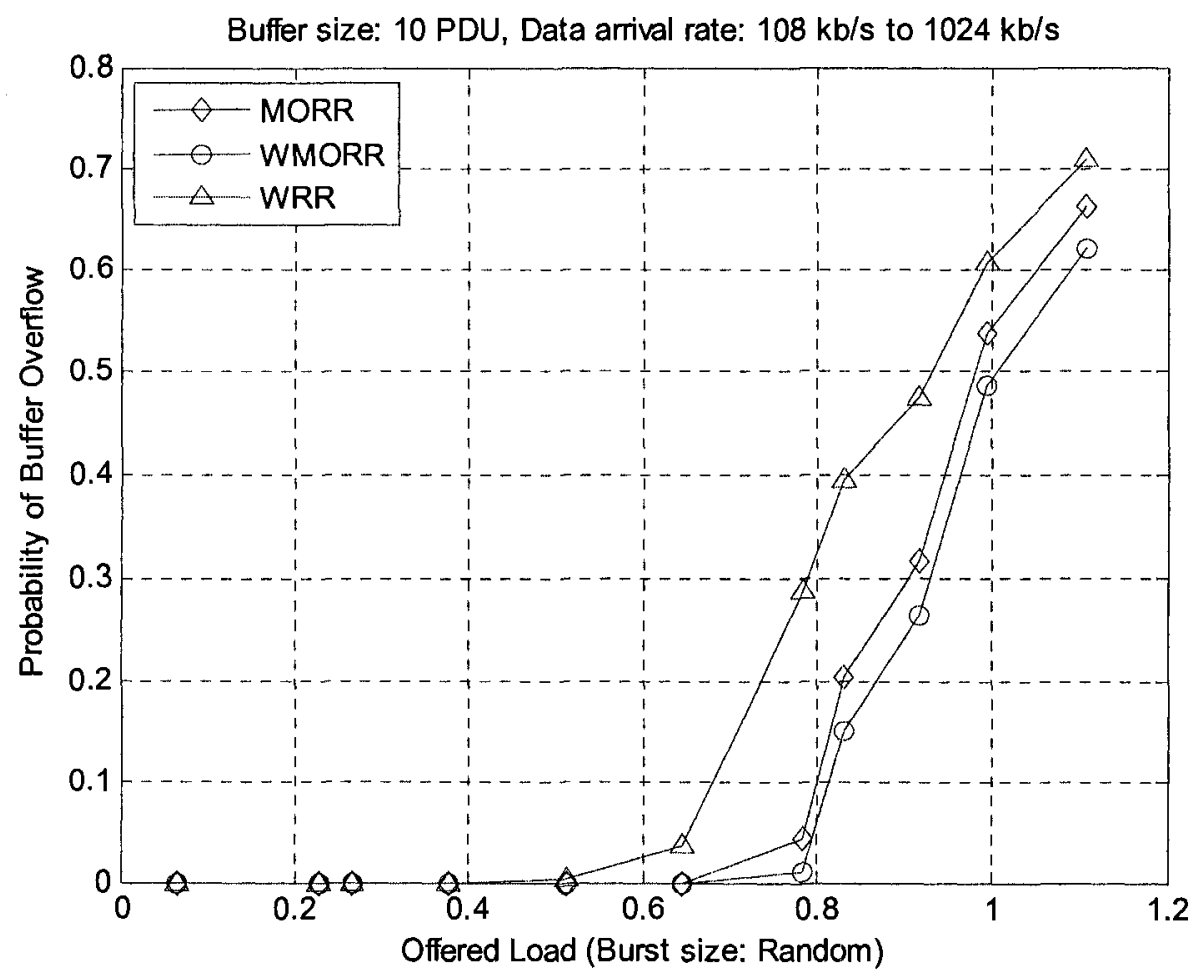

Figure 5.59. Probability of buffer overflow vs offered load for buffer size 10 at random burst. 


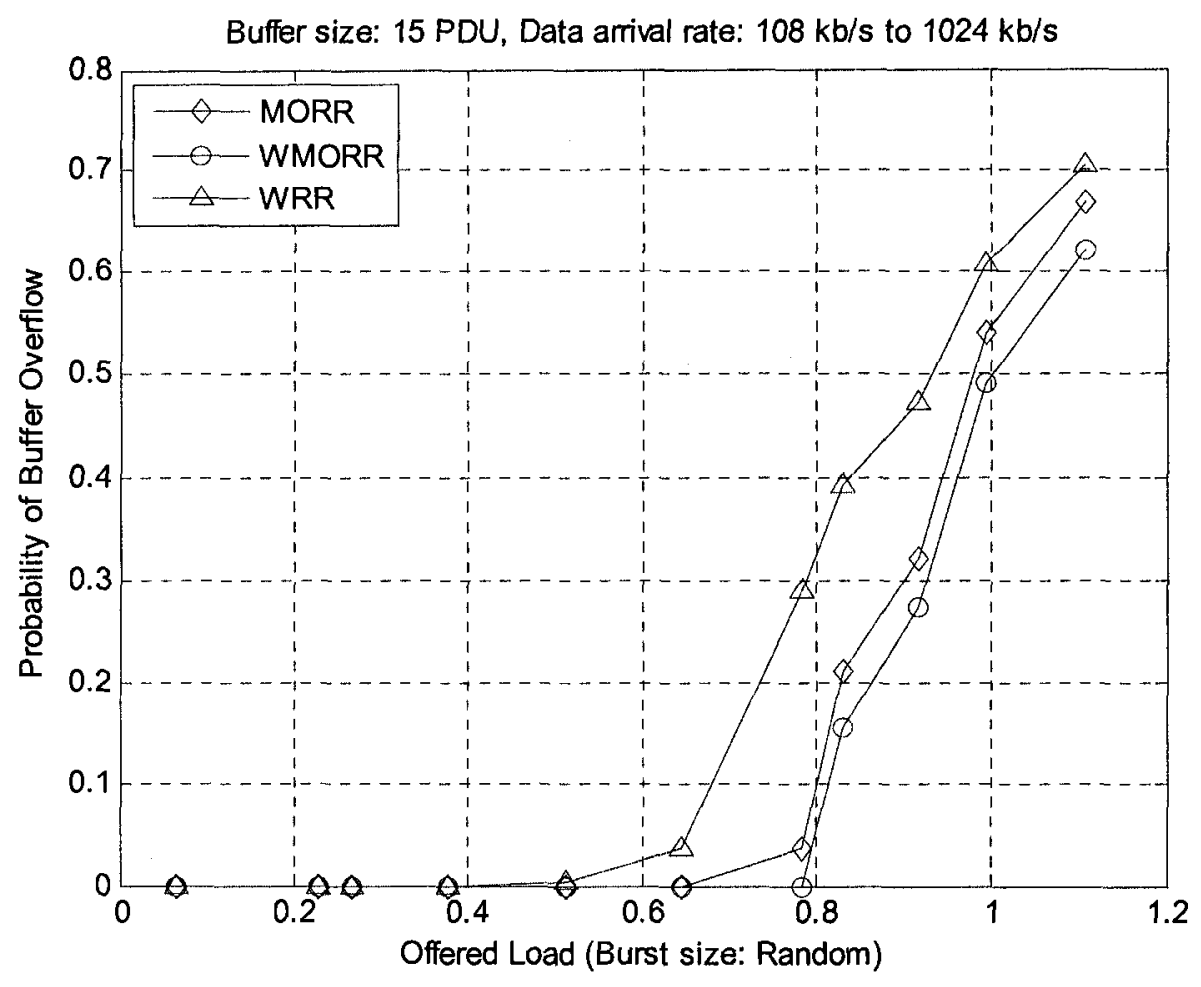

Figure 5.60. Probability of buffer overflow vs offered load for buffer size 15 at random burst.

Fig. 5.61, 5.62 and 5.63 shows the Probability of buffer overflow as a function of offered load for the buffer sizes 5,10 and 15 PDUs and the figures are for the MORR, WMORR and WRR resource allocation schemes respectively. In the fig. 5.61 and 5.62 when the load is between 0.6 and 0.8 buffer size 15 shows the lowest probability of overflow and the buffer size 5 shows the highest probability of overflow. But in Fig. 5.63 we do not notice any significat change in probabilty of overflow due to change in buffer size. 


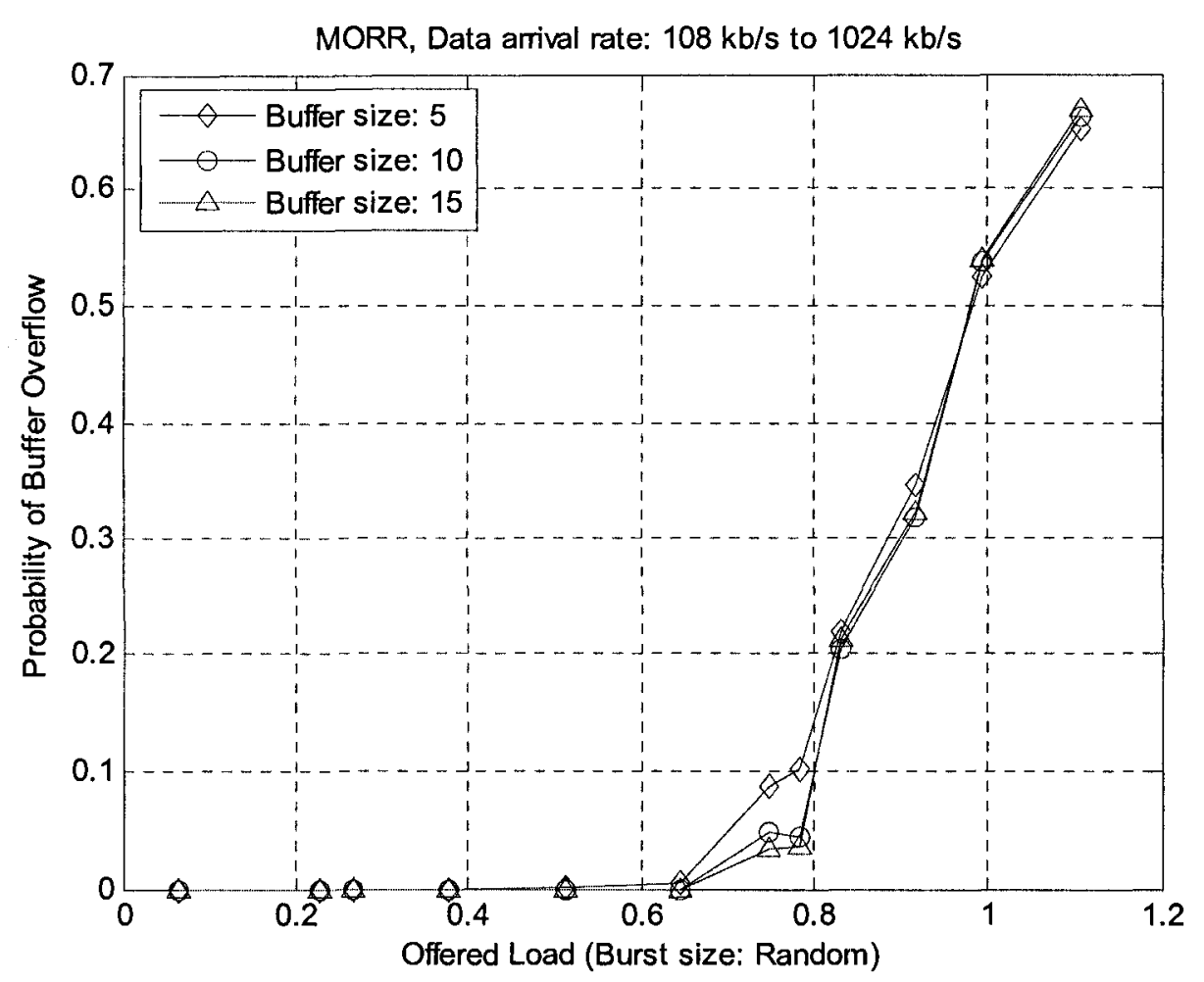

Figure 5.61. Probability of buffer overflow vs offered load for MORR scheme at random burst.

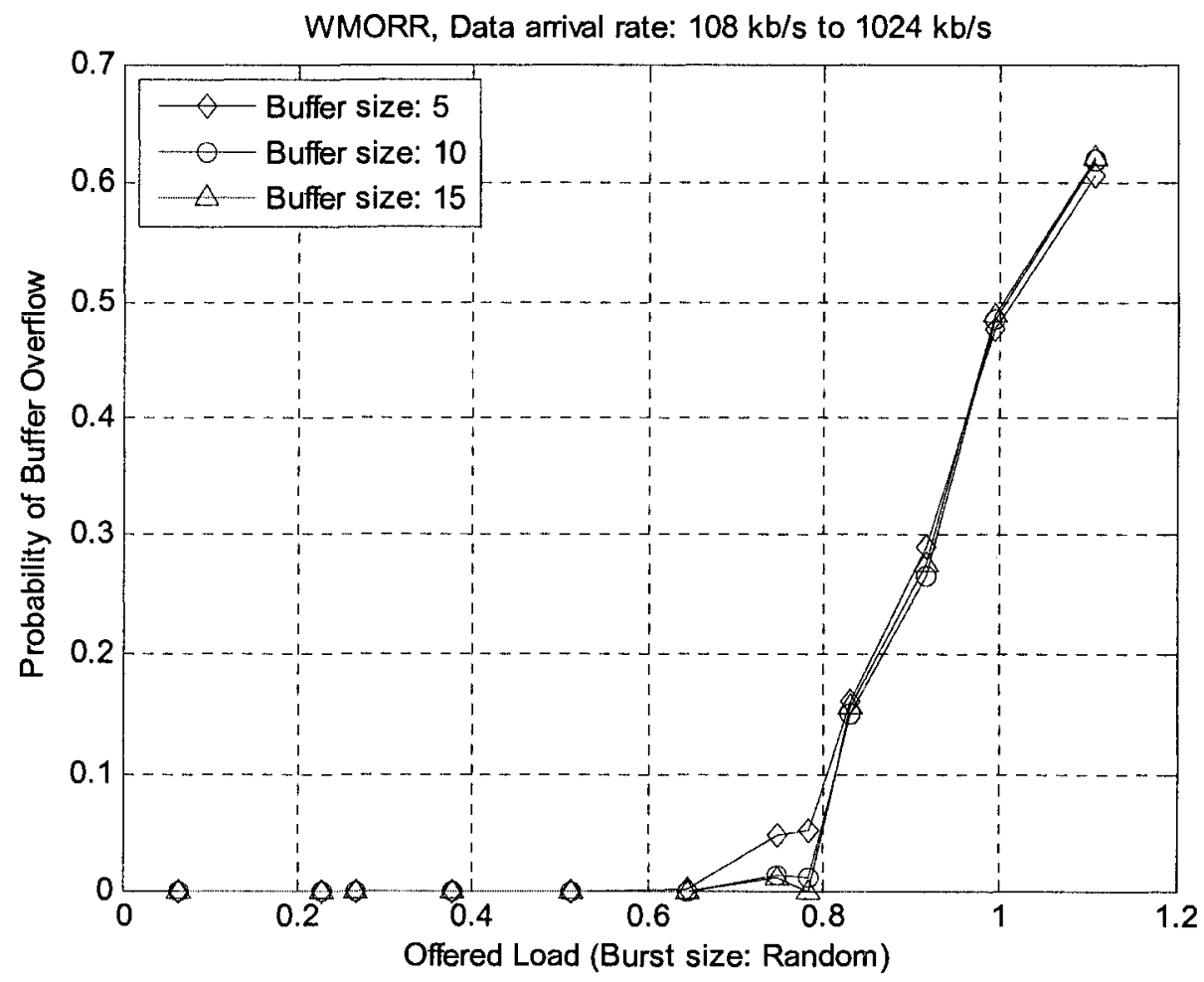

Figure 5.62. Probability of buffer overflow vs offered load for WMORR scheme at random burst. 


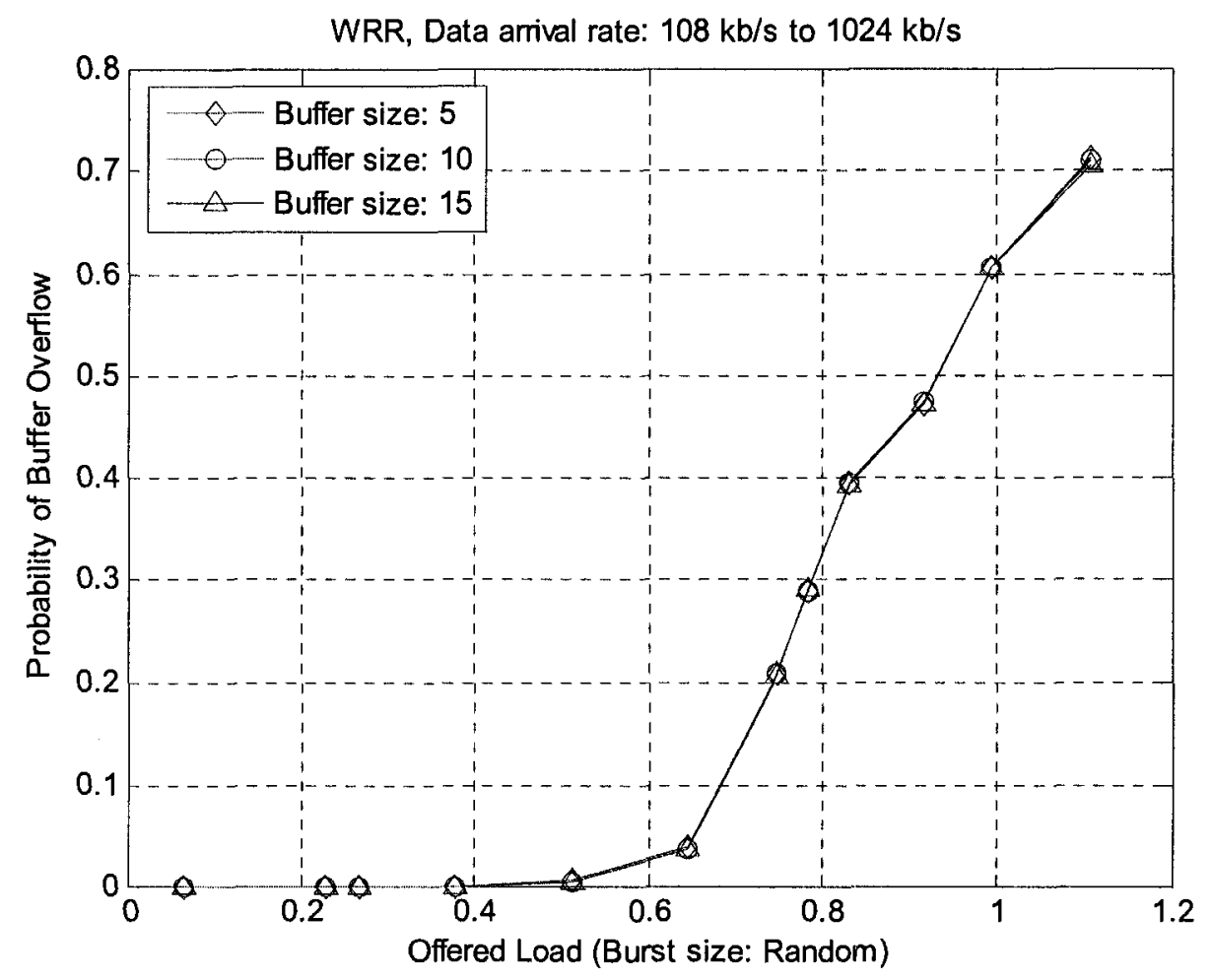

Figure 5.63. Probability of buffer overflow vs offered load for WRR scheme at random burst.

Variance in the buffer overflow is shown in the fig. 5.64, 5.65 and 5.66 as a function of offered load for the three resource allocation schemes and the figures represent the results when the buffer size is 5,10 and 15 PDUs respectively. For all the curves the variance of buffer overflow is zero for low value of offered load as buffer overflow is also zero (Fig. 5.58 to 5.63). But for higer value of offered load the varinace becomes large as the change in probability of buffer overflow is large at high load. 


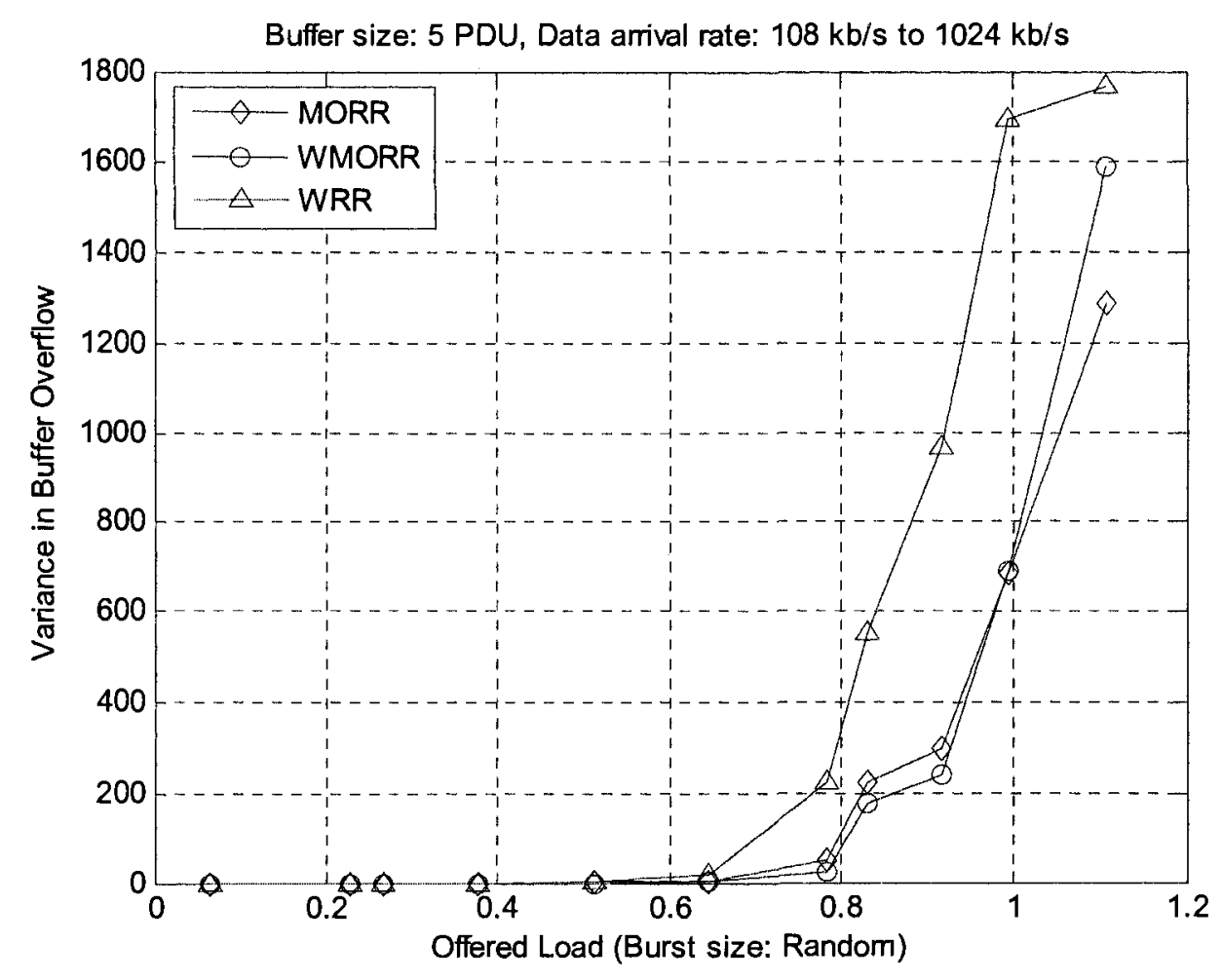

Figure 5.64. Variance in buffer overflow vs offered load for buffer size 5 at random burst.

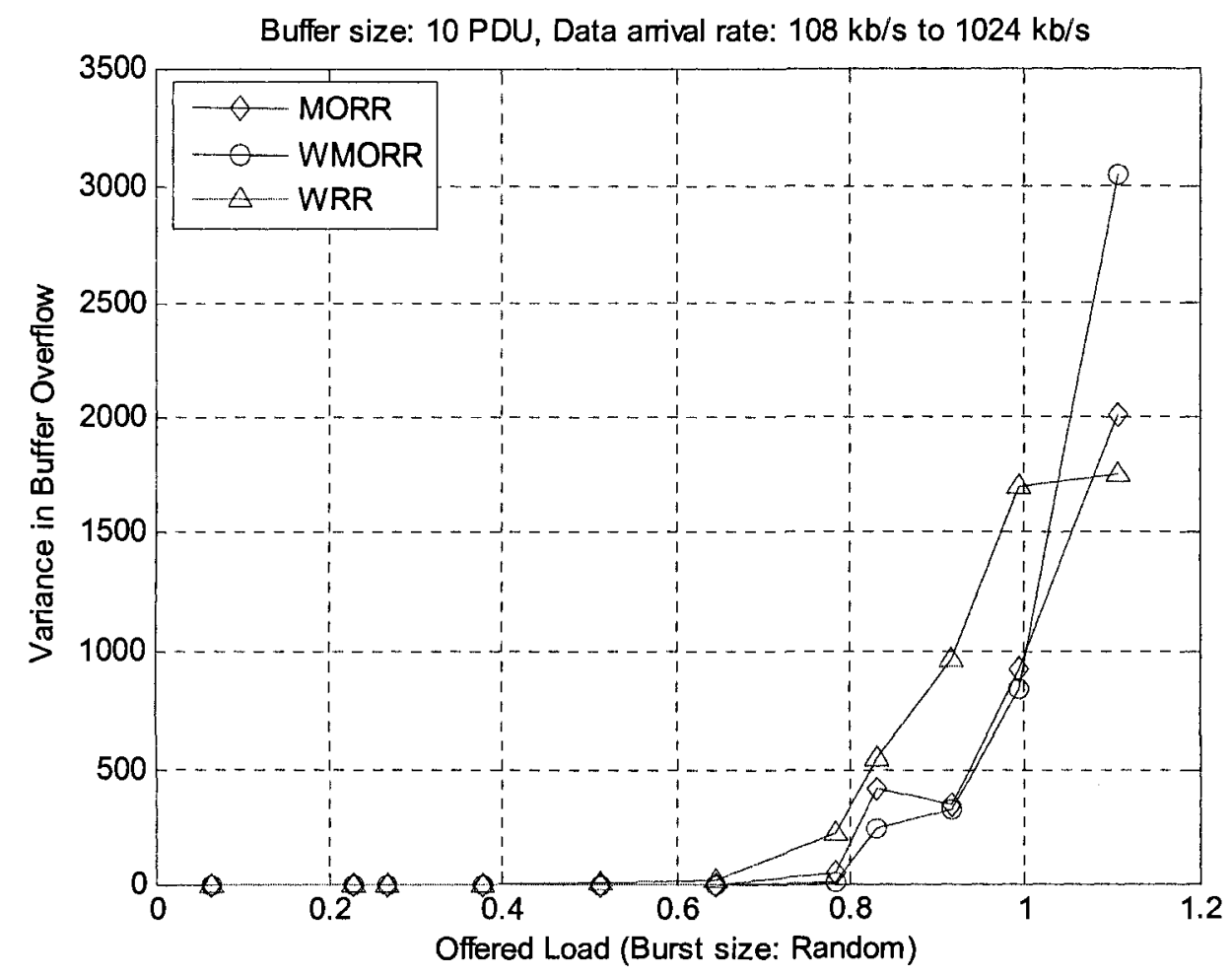

Figure 5.65. Variance in buffer overflow vs offered load for buffer size10 at random burst. 


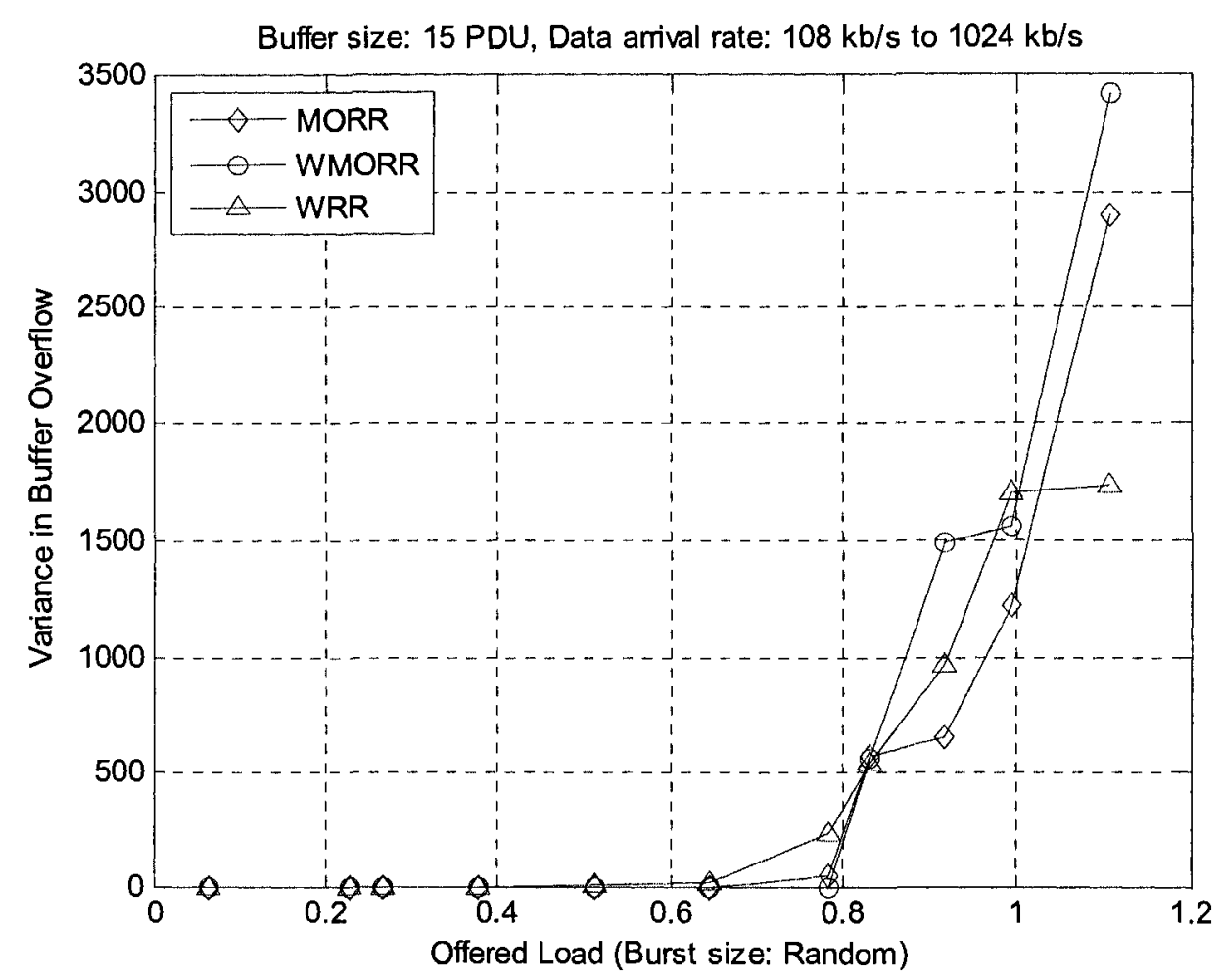

Figure 5.66. Variance in buffer overflow vs offered load for buffer size 15 at random burst.

\section{- Average PDU delivery time and its variance}

Fig. 5.67, 5.68 and 5.69 depicts average PDU delivery time in seconds as a function of offered load for the three resource allocation policies, where the buffer size is 5, 10 and 15 PDUs respectively. In the figures MORR and WMORR schemes show lower PDU delivery time compared to WRR scheme. Fow low value of offerred load the delivery time is low and remains constant for all the allocation policy because PDU contant in buffer is also constant (Fig. 5.49 to 5.54) and PDUs spend very small amount of time in the buffer. But when the system load increases PDU service rate becomes lower than the arrival rate and PDUs have to wait long in the buffer to get the transmission opportunity. 


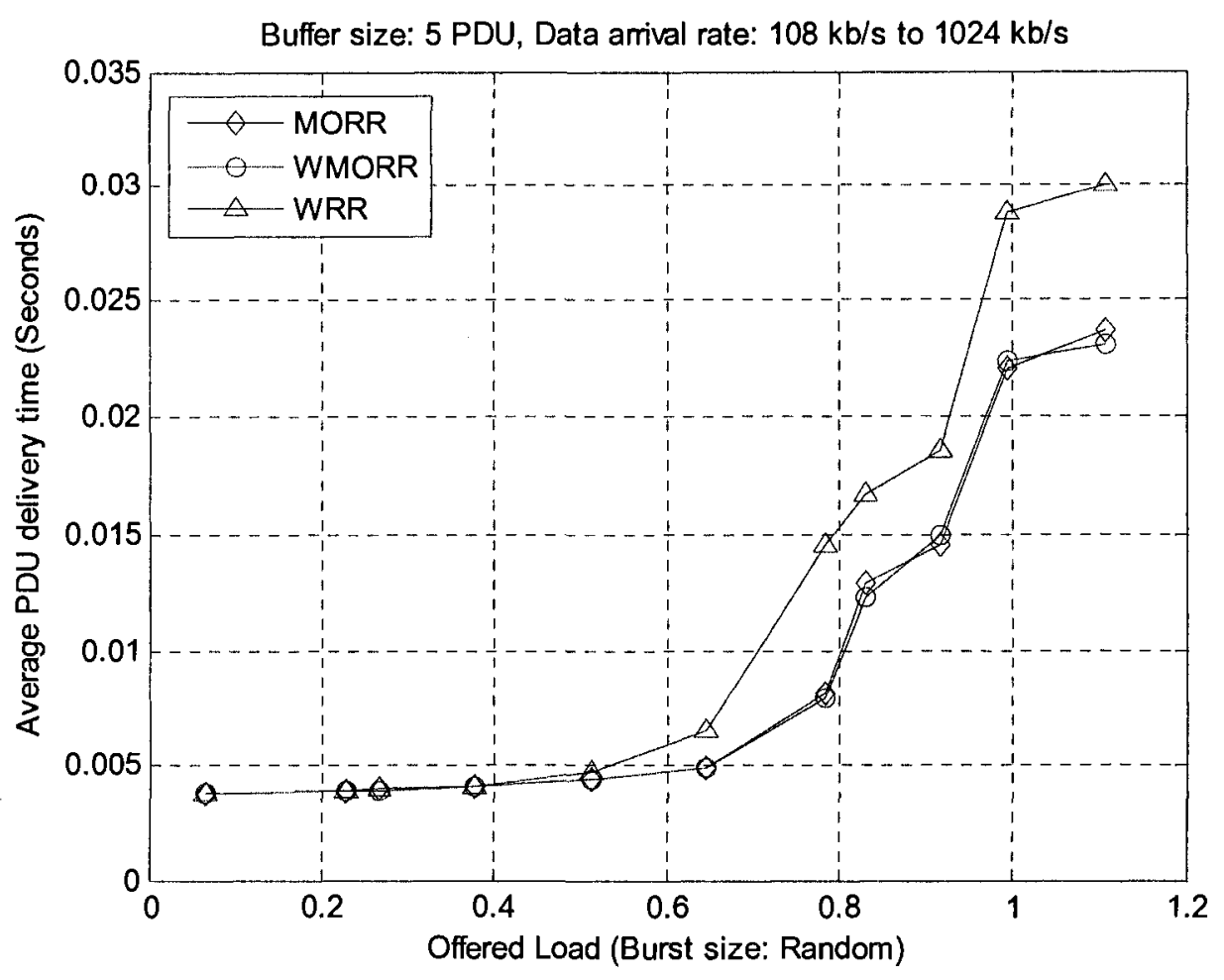

Figure 5.67. Average PDU delivery time vs offered load for buffer size 5 at random burst.

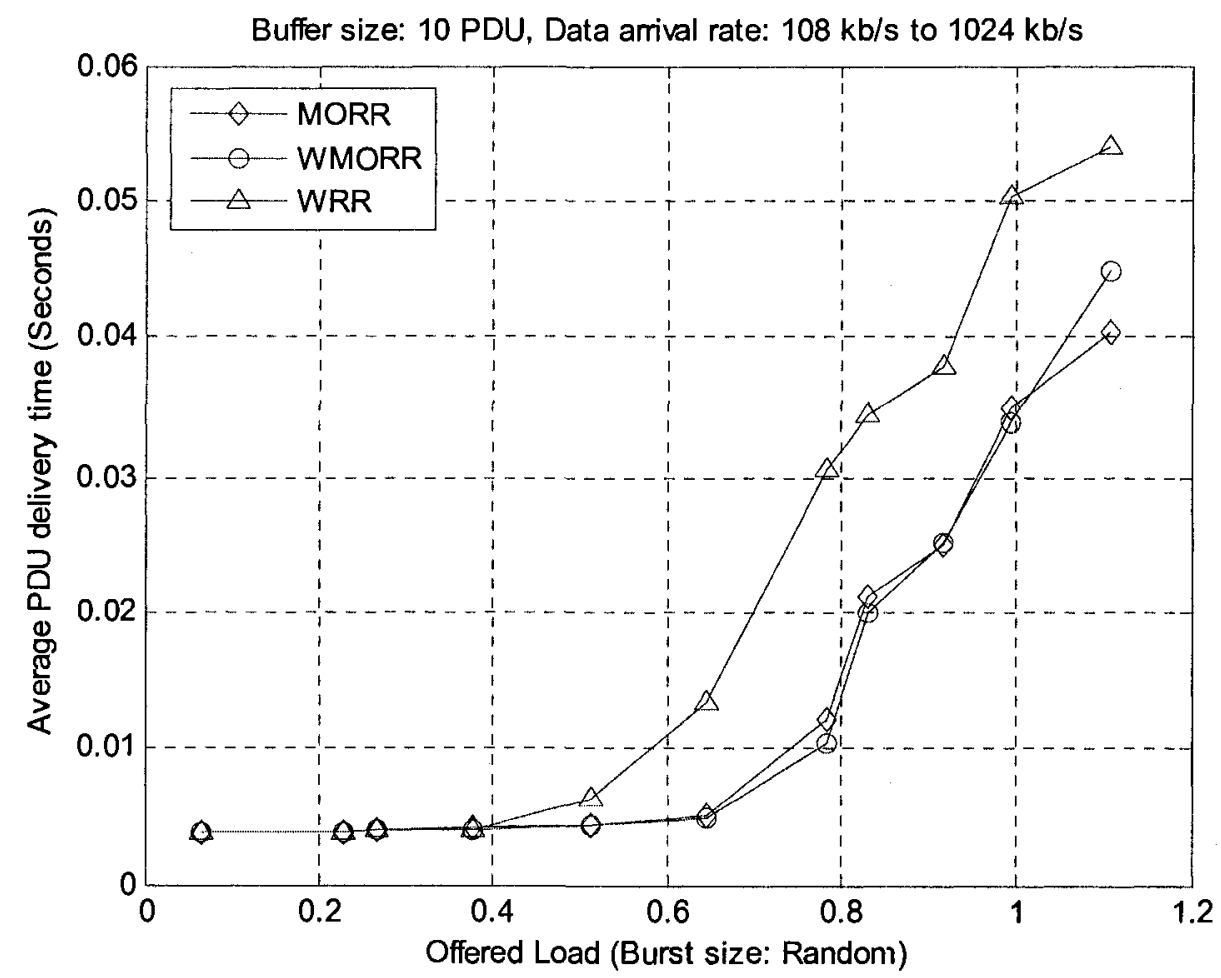

Figure 5.68. Average PDU delivery time vs offered load for buffer size 10 at random burst. 


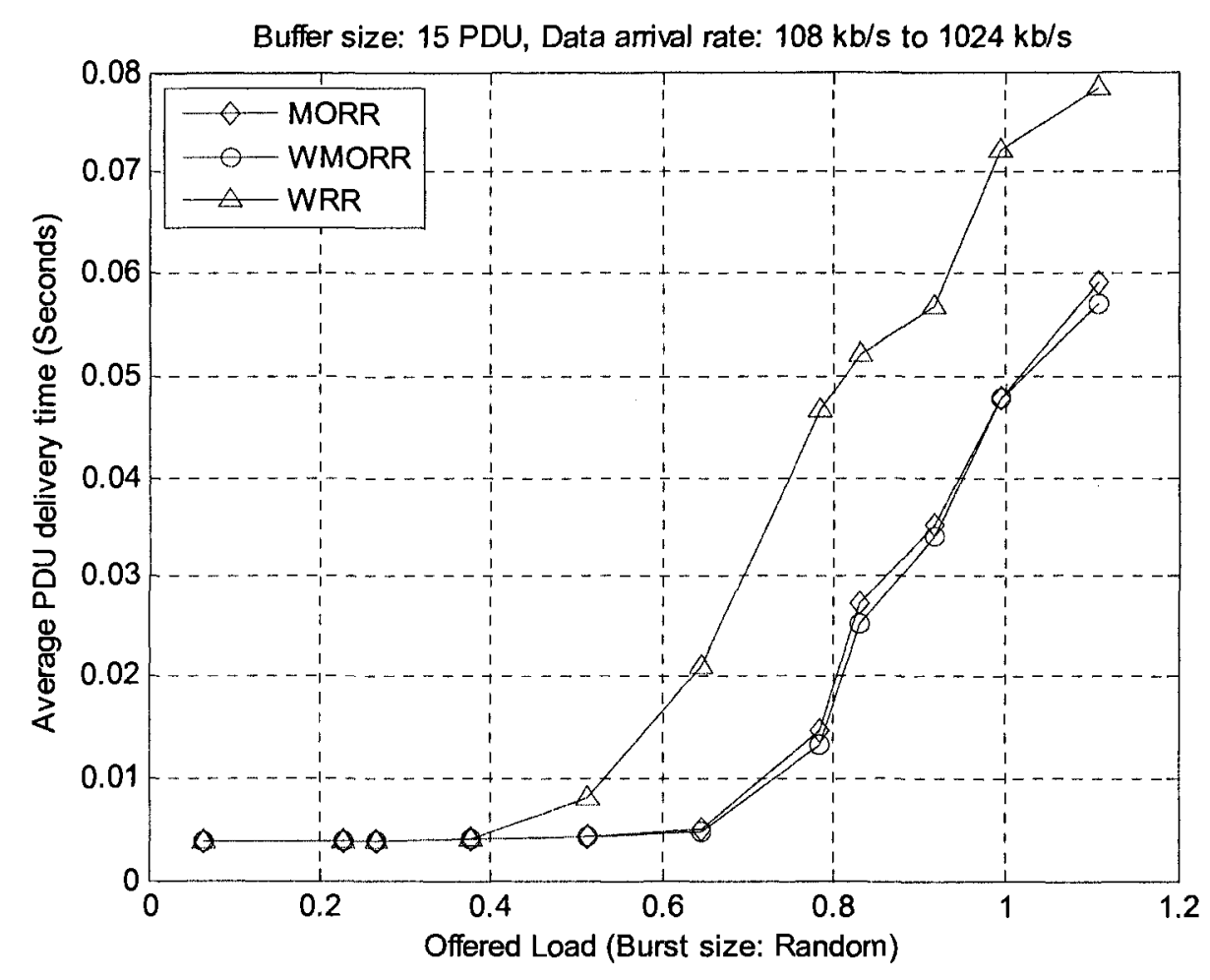

Figure 5.69. Average PDU delivery time vs offered load for buffer size 15 at random burst.

Fig. 5.70, 5.71 and 5.72 show average PDU delivery time in seconds as a function of offered load for the buffer sizes 5, 10 and 15 PDUs and the figures are for the MORR, WMORR and WRR resource allocation schemes respectively. All the figures show that with the increase of buffer size average delivery time also increases as PDUs have to wait long in the buffer to get the transmission opportunity. In the figures we also notice that for load lower than 0.7 for MORR and WMORR and 0.4 for WRR scheme PDU delivery time remains constant and it does not depend on buffer size. 


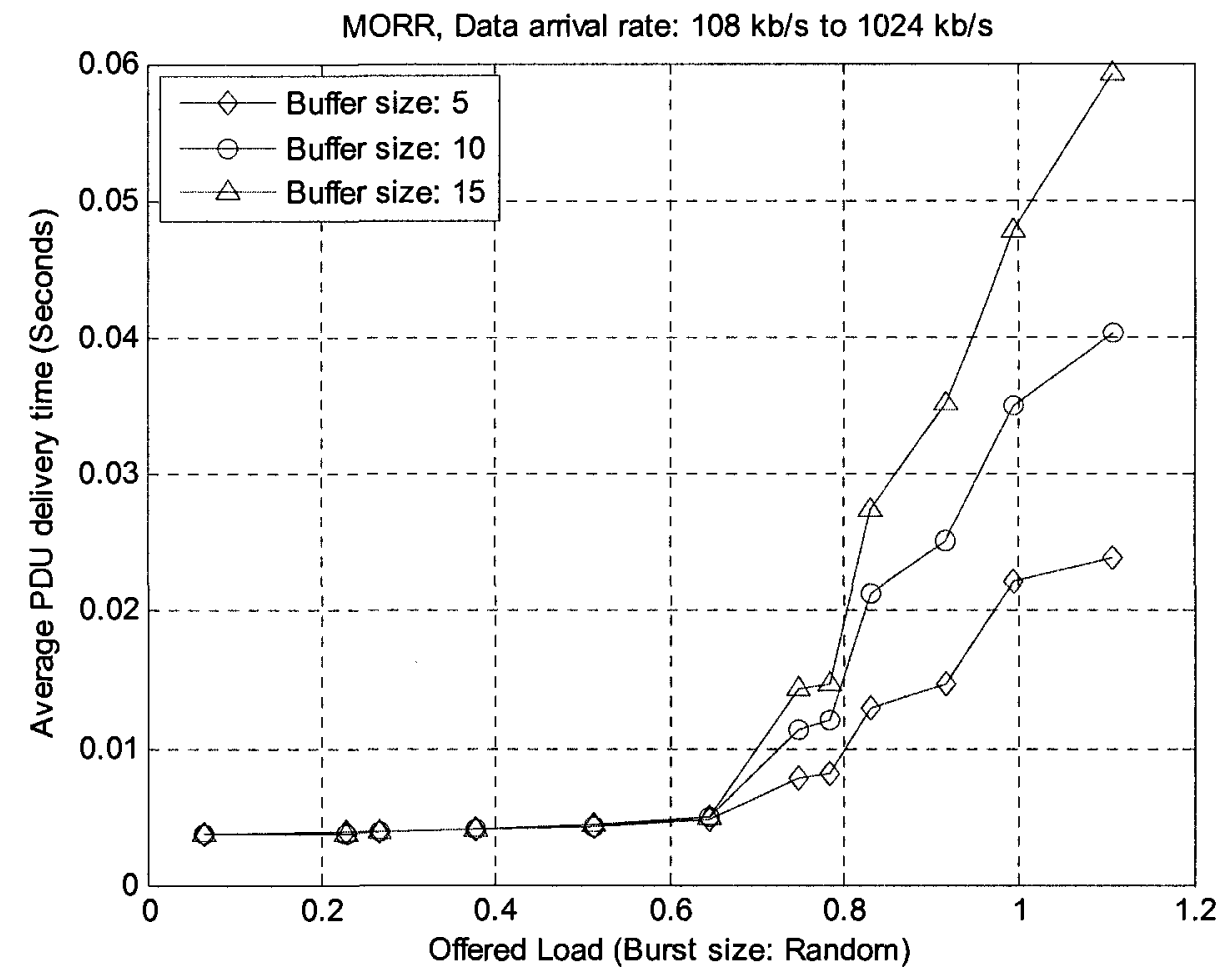

Figure 5.70. Average PDU delivery time vs offered load for MORR scheme at random burst.

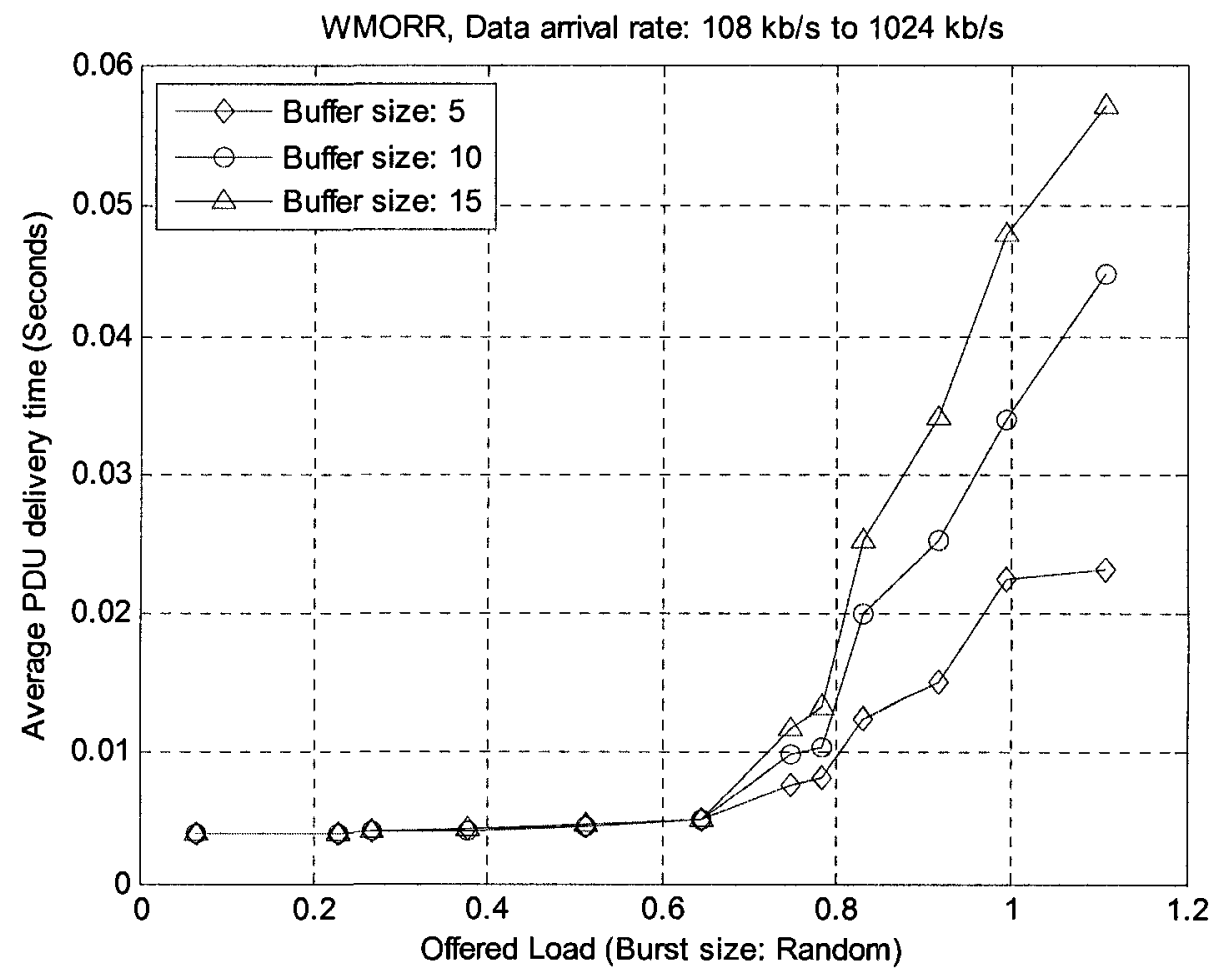

Figure 5.71. Average PDU delivery time vs offered load for WMORR scheme at random burst. 


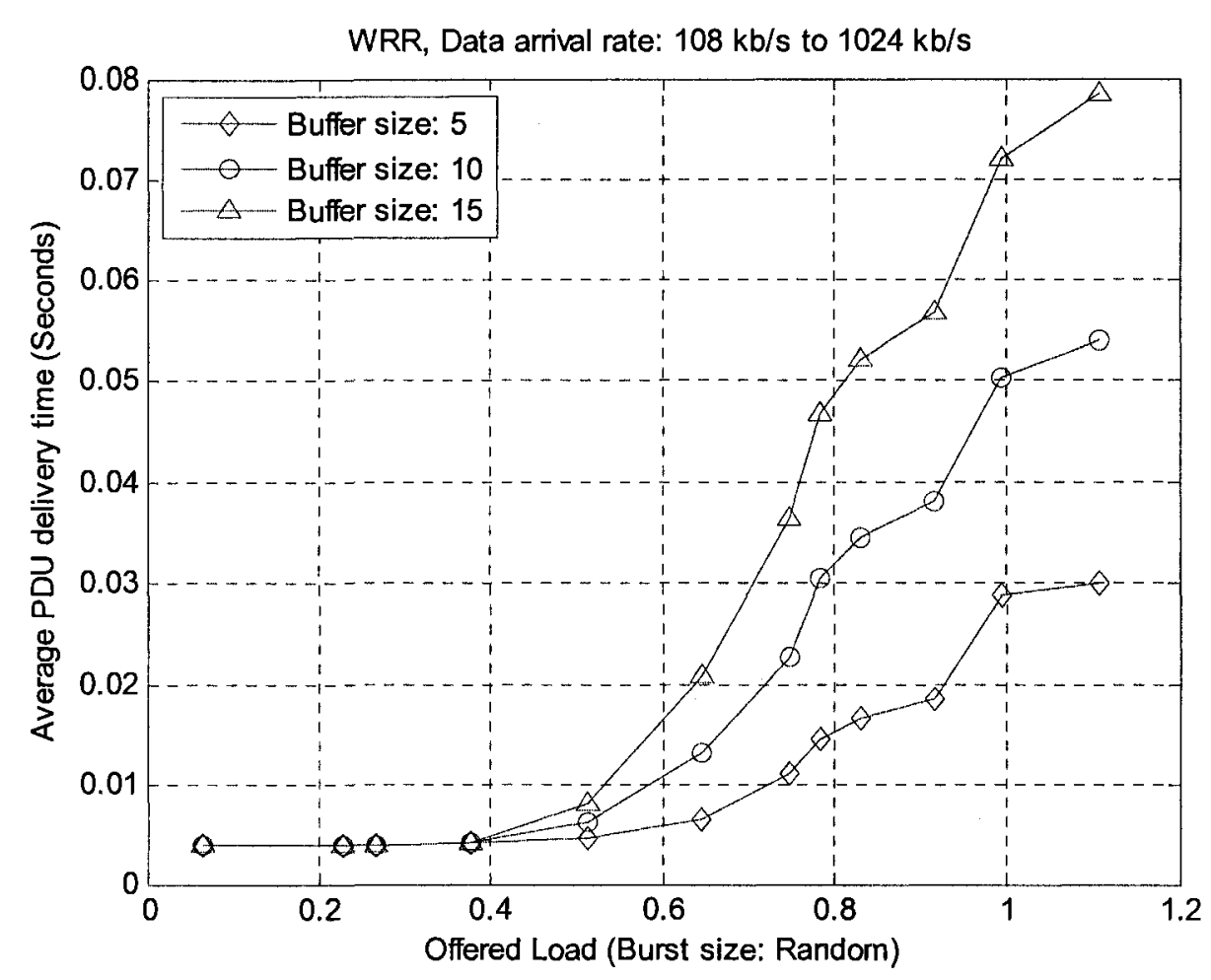

Figure 5.72. Average PDU delivery time vs offered load for WRR scheme at random burst.

Variance in the PDU delivery time is shown in the fig. 5.73, 5.74 and 5.75 as a function of offered load for the three resource allocation schemes and the figures represent the results when the buffer size is 5,10 and 15 PDUs respectively. For all the curves the variance of PDU delivery time is zero when offered load is below 0.8 . But for very high value of offered load variance in delivery time becomes high. We also notice that the varince for WRR scheme is very hgih and it is out of the range used in the figure. 


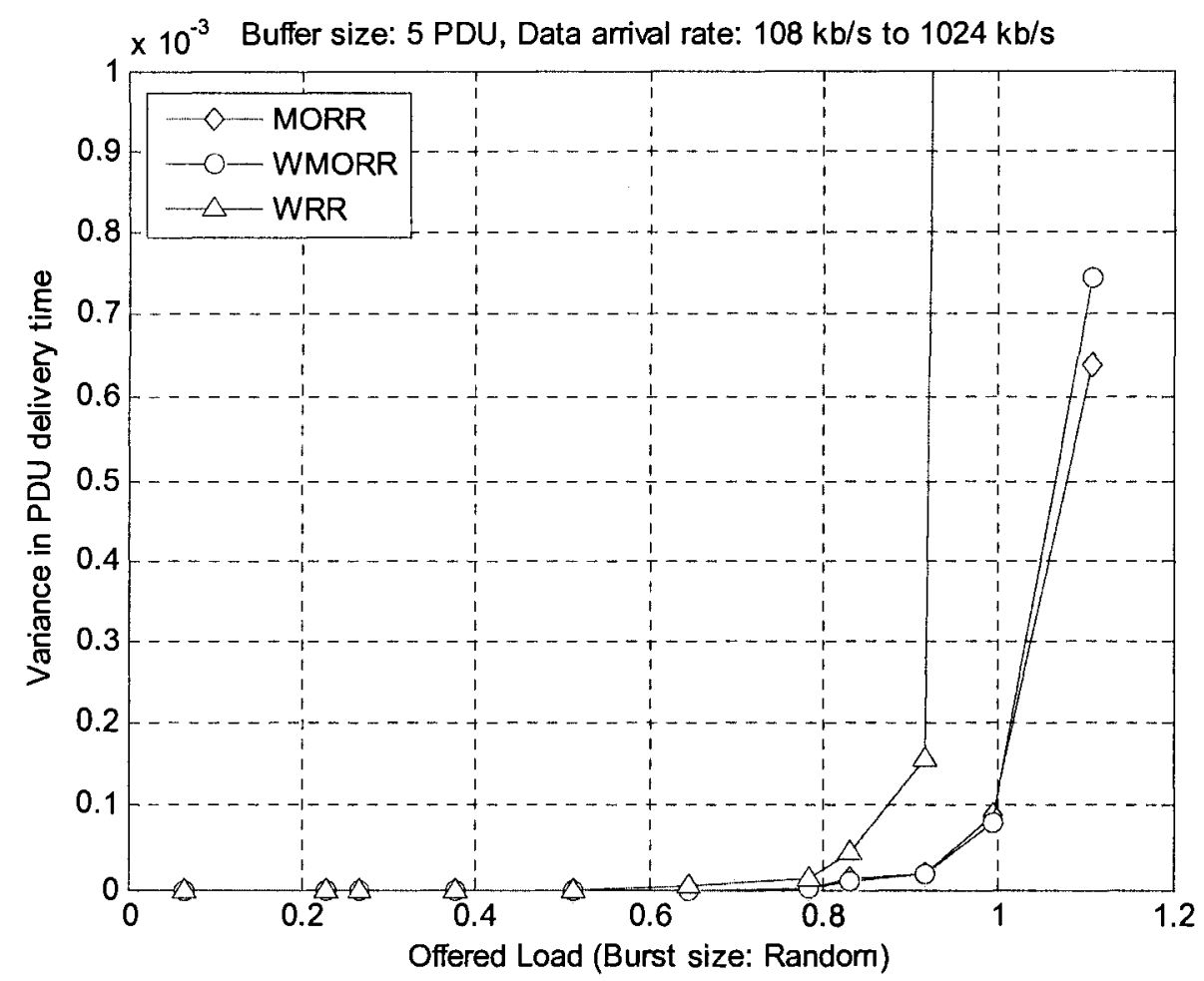

Figure 5.73. Variance in PDU delivery time vs offered load for buffer size 5 at random burst.

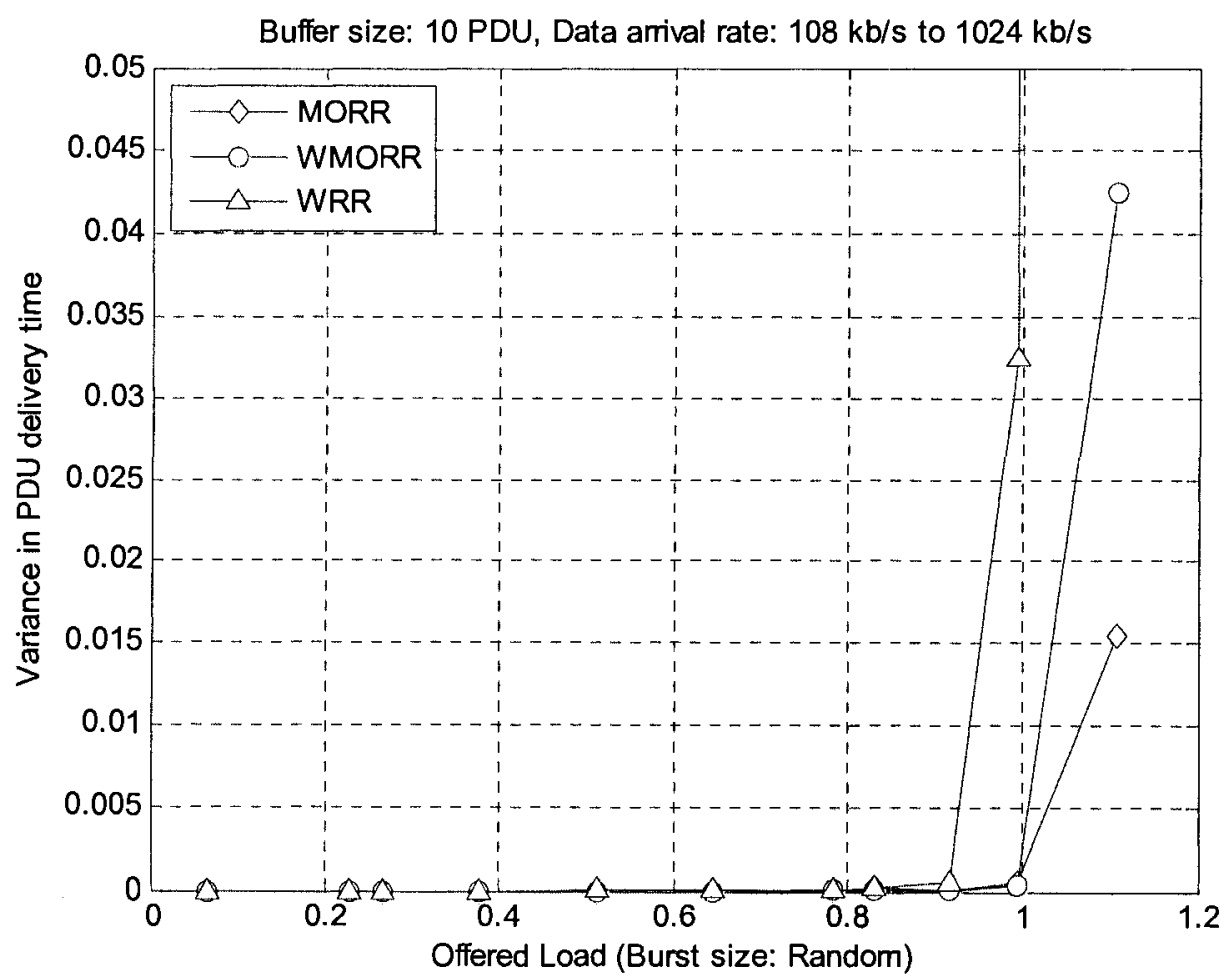

Figure 5.74. Variance in PDU delivery time vs offered load for buffer size 10 at random burst. 


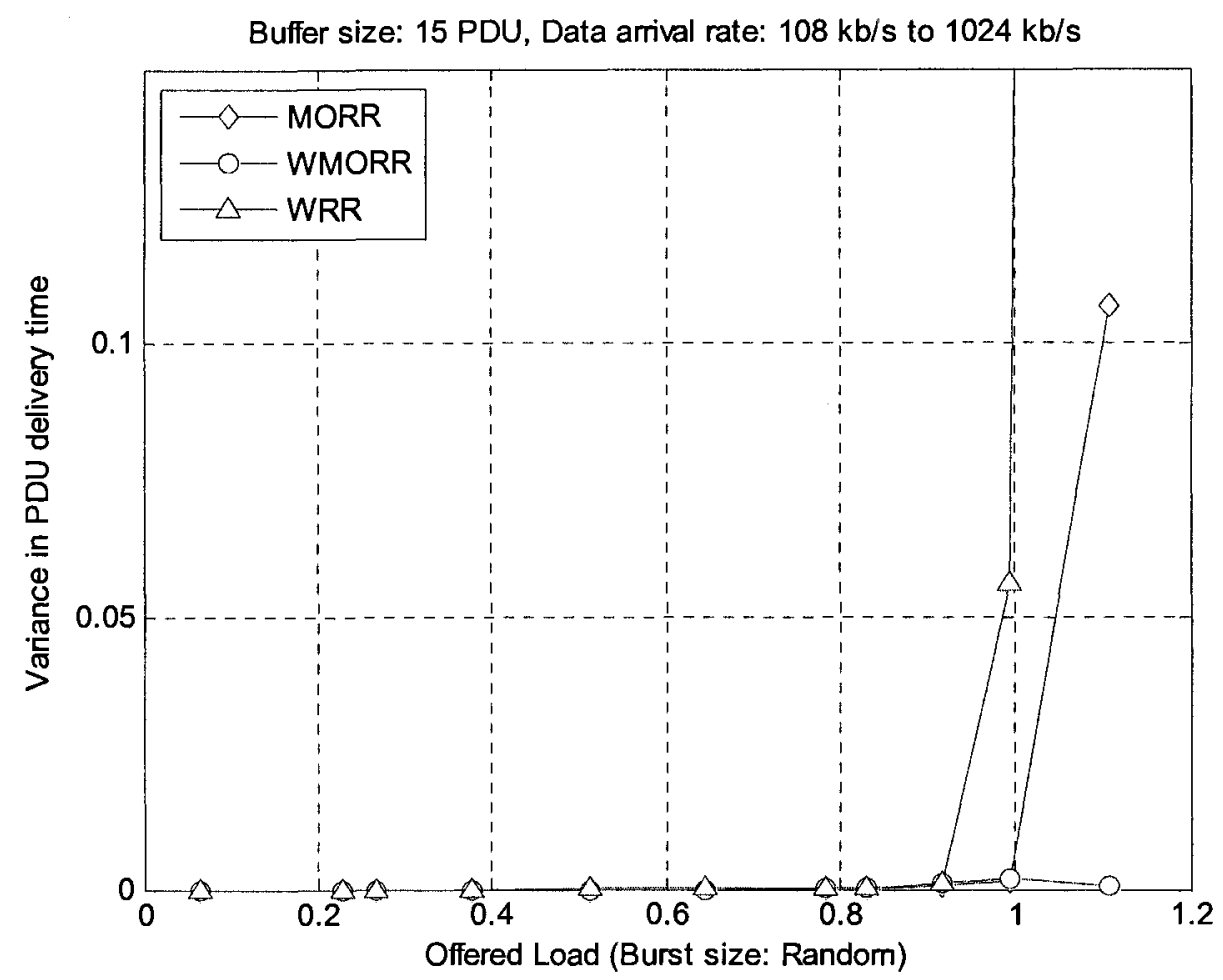

Figure 5.75. Variance in PDU delivery time vs offered load for buffer size 15 at random burst.

\section{- Average Overhead in Uplink Phase and its variance}

Fig. 5.76, 5.77 and 5.78 depict the average overhead (Percentage of total uplink time) in uplink phase as a function of offered load for the three resource allocation policies, where buffer size is 5, 10 and 15 PDUs respectively. The figures show that the MORR and WMORR schemes have low overhead compared to the WRR allocation scheme. For low value of offered load the uplink overhead is large as all the arrived PDU gets the transmission oppourtunity and overhead of all the user add up to give a large overhead. But as the load increases arrival rate beomes higher than the service rate and very few users get the transmission opportunity. This results a very low overhead for MORR and WMORR scheme as when ever an user is visited, maximum capacity is assigned to it according to these schemes. But for the WRR scheme as all the user buffer is visited during a TDMA frame, ovherhead of all the user adds up and the summaion continue to increase with the increase of load. 


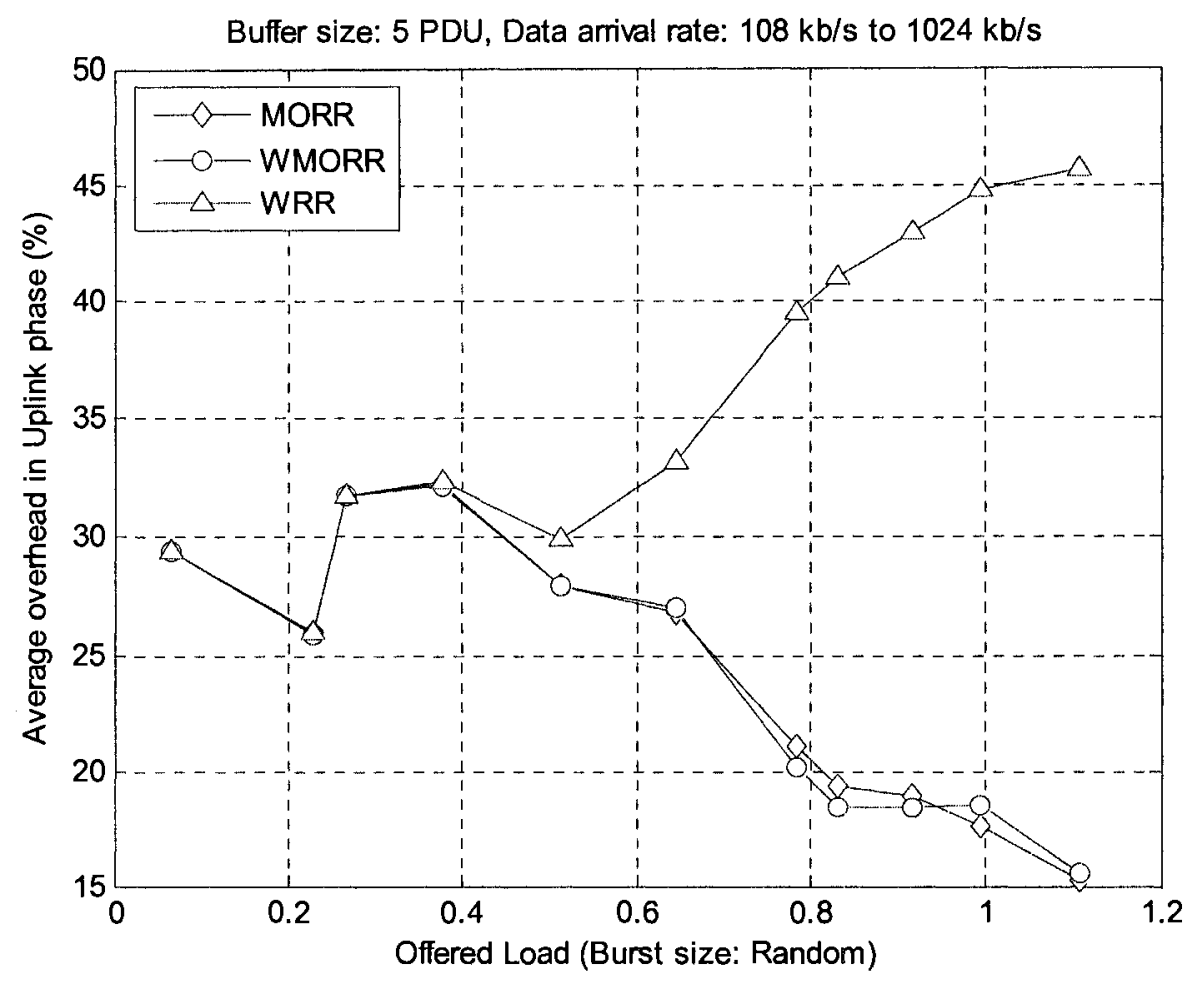

Figure 5.76. Average overhead in uplink phase vs offered load for buffer size 5 at random burst.

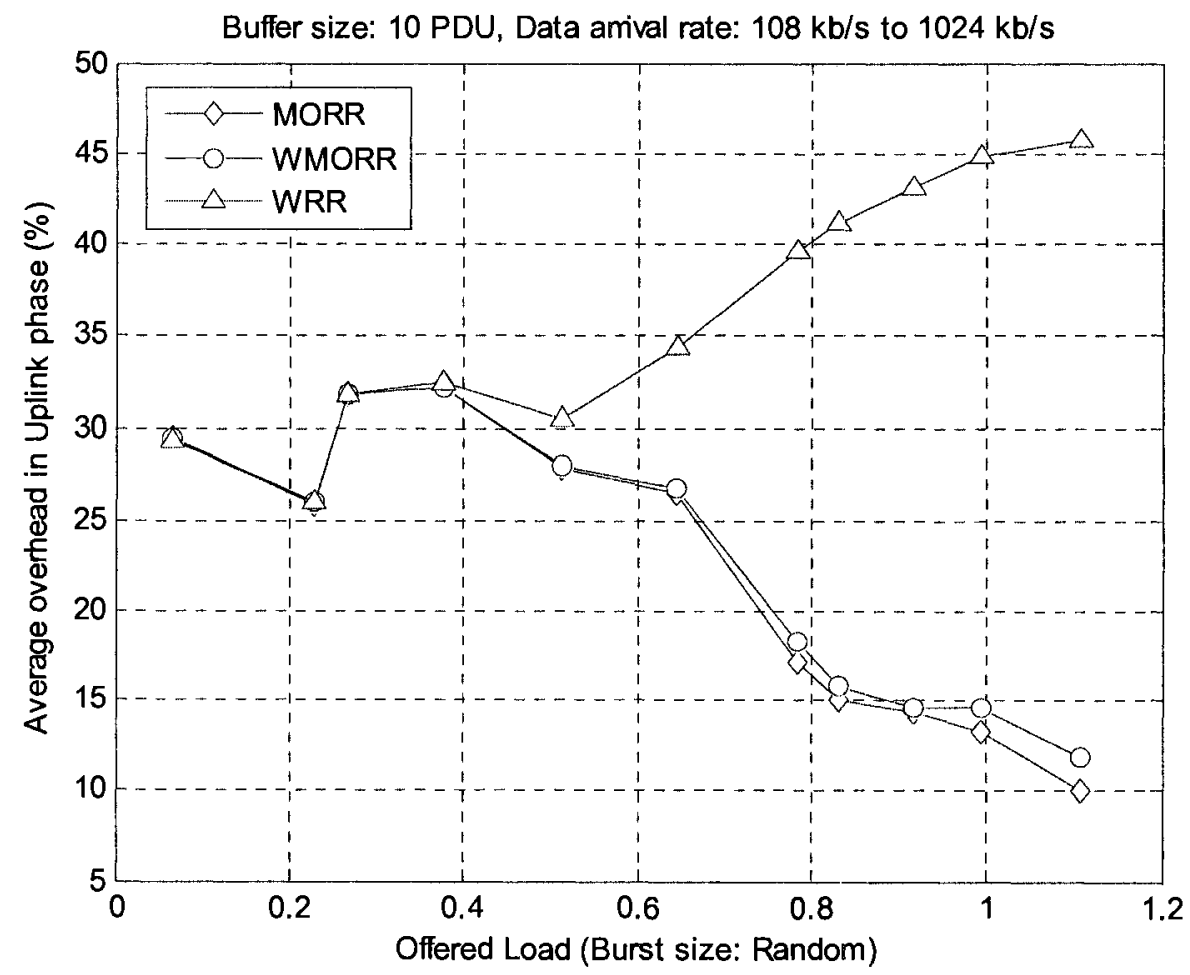

Figure 5.77. Average overhead in uplink phase vs offered load for buffer size 10 at random burst. 


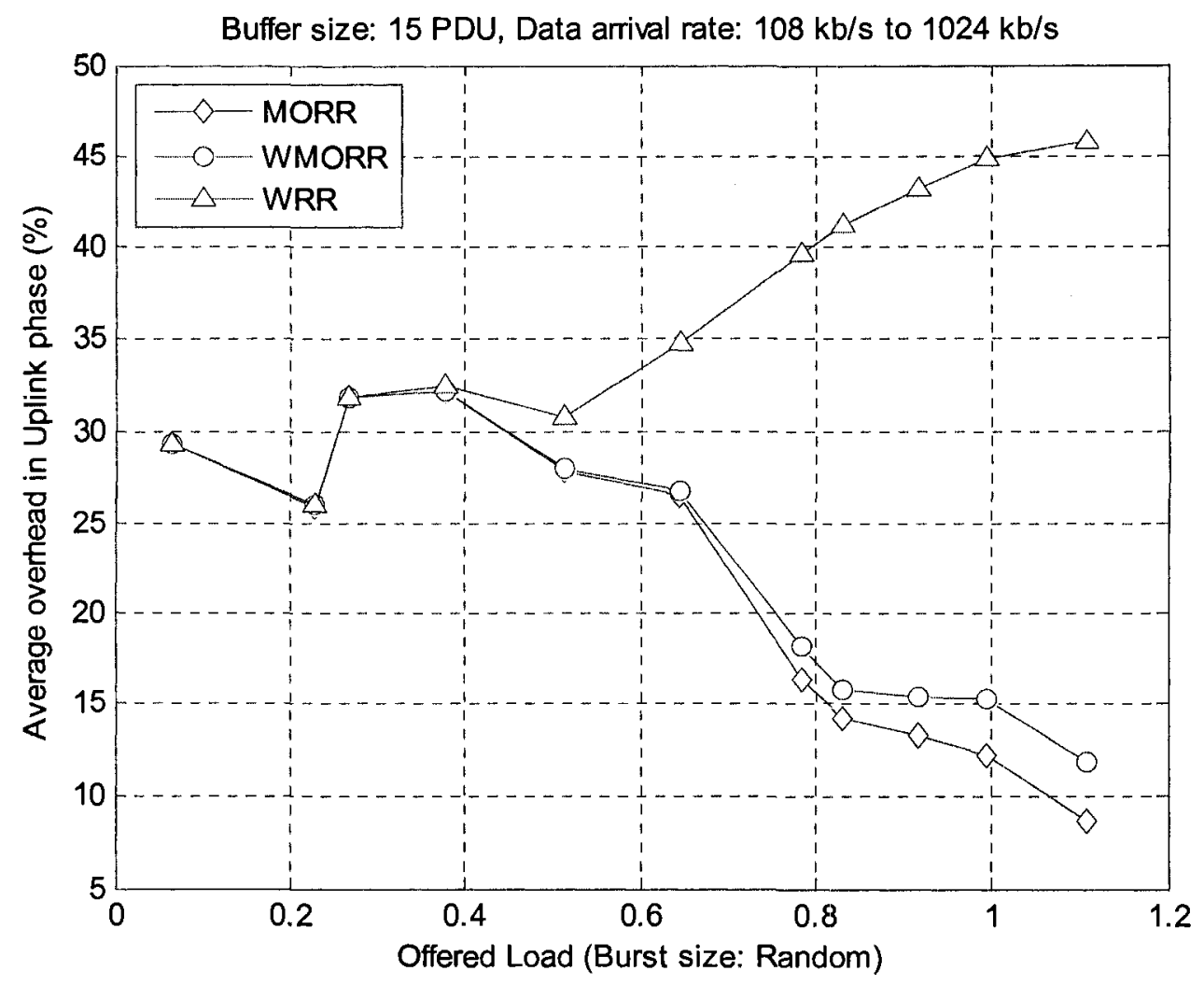

Figure 5.78. Average overhead in uplink phase vs offered load for buffer size 15 at random burst.

Fig. 5.79, 5.80 and 5.81 shows the average overhead in uplink phase (Percentage of total uplink time) as a function of offered load for buffer sizes 5, 10 and 15 PDUs and the figures are for the MORR, WMORR and WRR resource allocation schemes respectively. We notice that for WMORR and MORR schemes overhead decreases with the increase of buffer size at high load. This change in overhead corresponds to the allocaion scheme, as for low load all MTs are scheduled for transmission in the TDMA frame but at high load a few of the MTs are scheduled for transmission. Also in these schemes, at high load the number of MTs to be scheduled for transmission depends on the buffer occupancy i.e. buffer size of the MTs. For the WRR scheme overhead remains same for all buffer sizes because in this scheme all the MTs are scheduled for transmission in the TDMA frame and overhead does not depend on the buffer size 


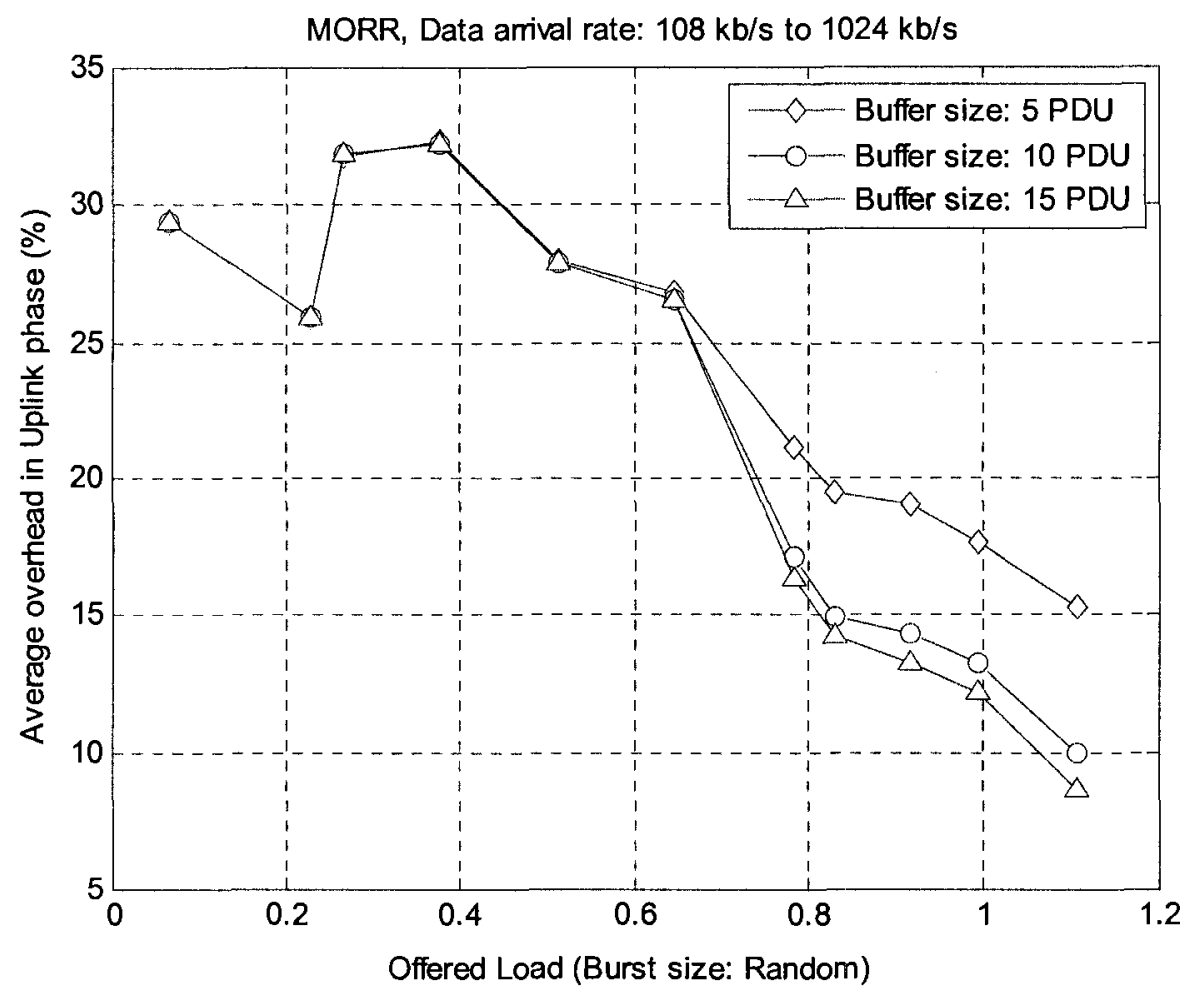

Figure 5.79. Average overhead in uplink phase vs offered load for MORR scheme at random burst.

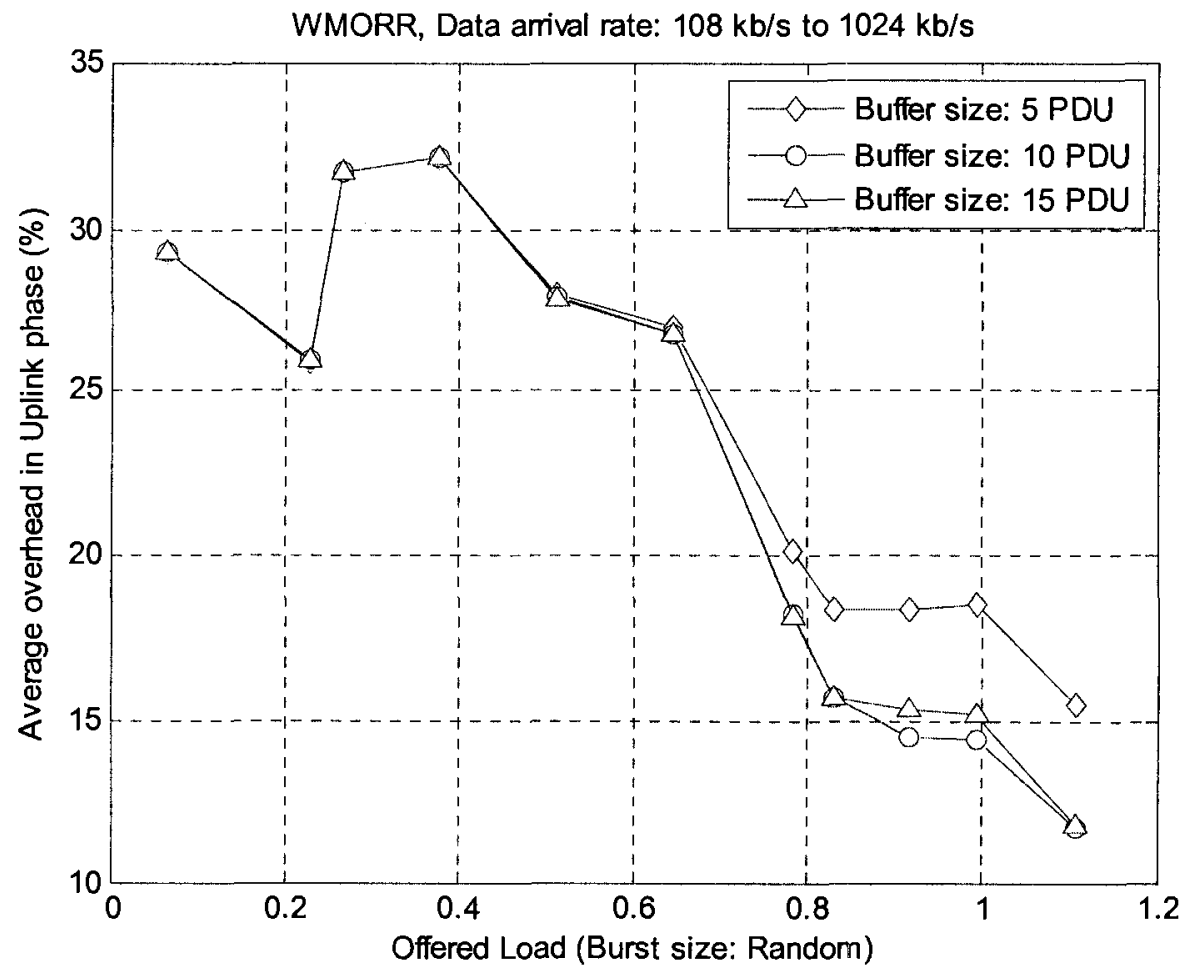

Figure 5.80. Average overhead in uplink phase vs offered load for WMORR scheme at random burst. 


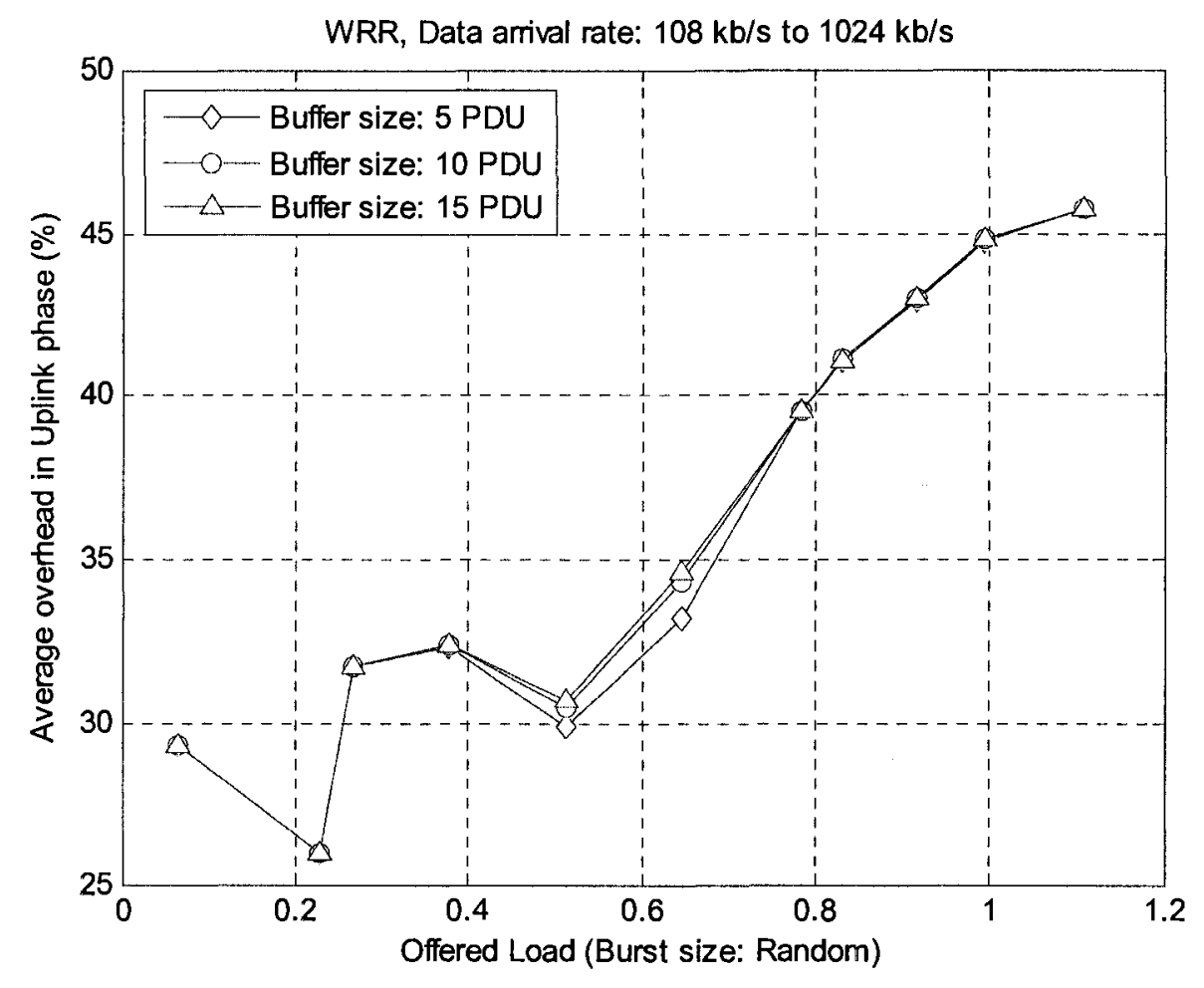

Figure 5.81. Average overhead in uplink phase vs offered load for WRR scheme at random burst.

Variance in overhead of uplink phase is shown in fig. 5.82, 5.83 and 5.84 as a function of offered load for the three resource allocation schemes and the figures represent the results when the buffer size is 5,10 and 15 PDUs respectively. We notice that change in variance corresponds to the change in the curves of fig. $5.76-5.81$. 


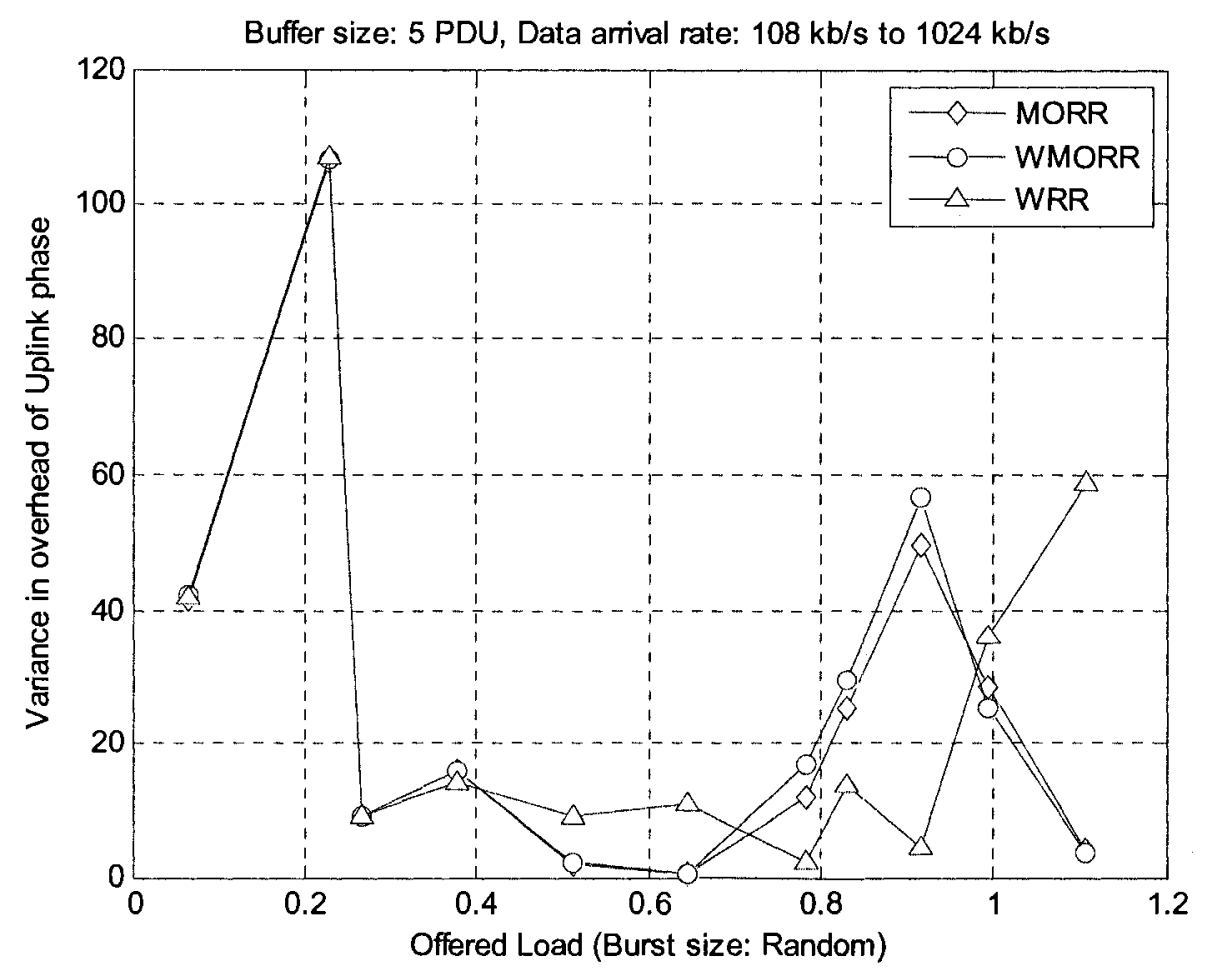

Figure 5.82. Variance in overhead of uplink phase vs offered load for buffer size 5 at random burst.

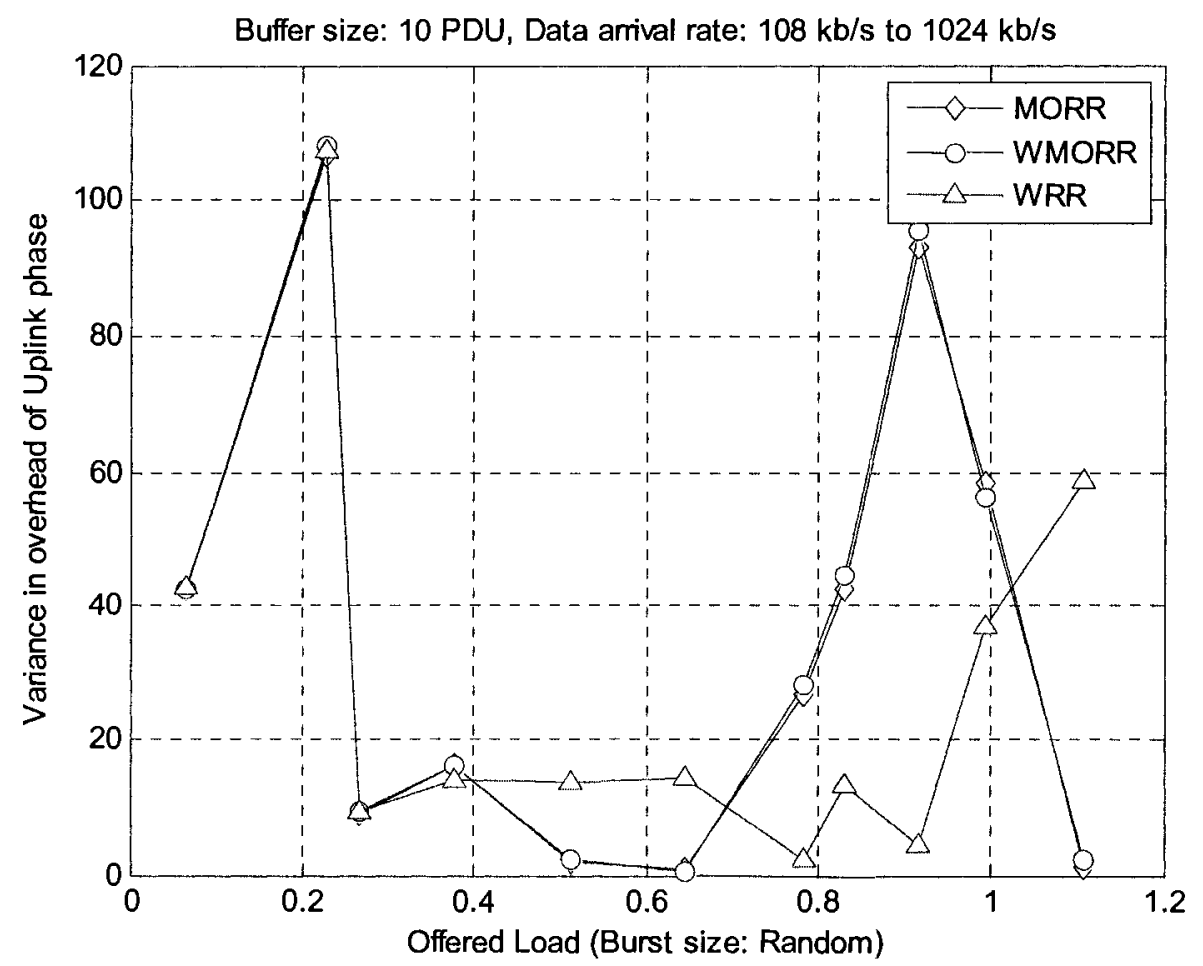

Figure 5.83. Variance in overhead of uplink phase vs offered load for buffer size 10 at random burst. 


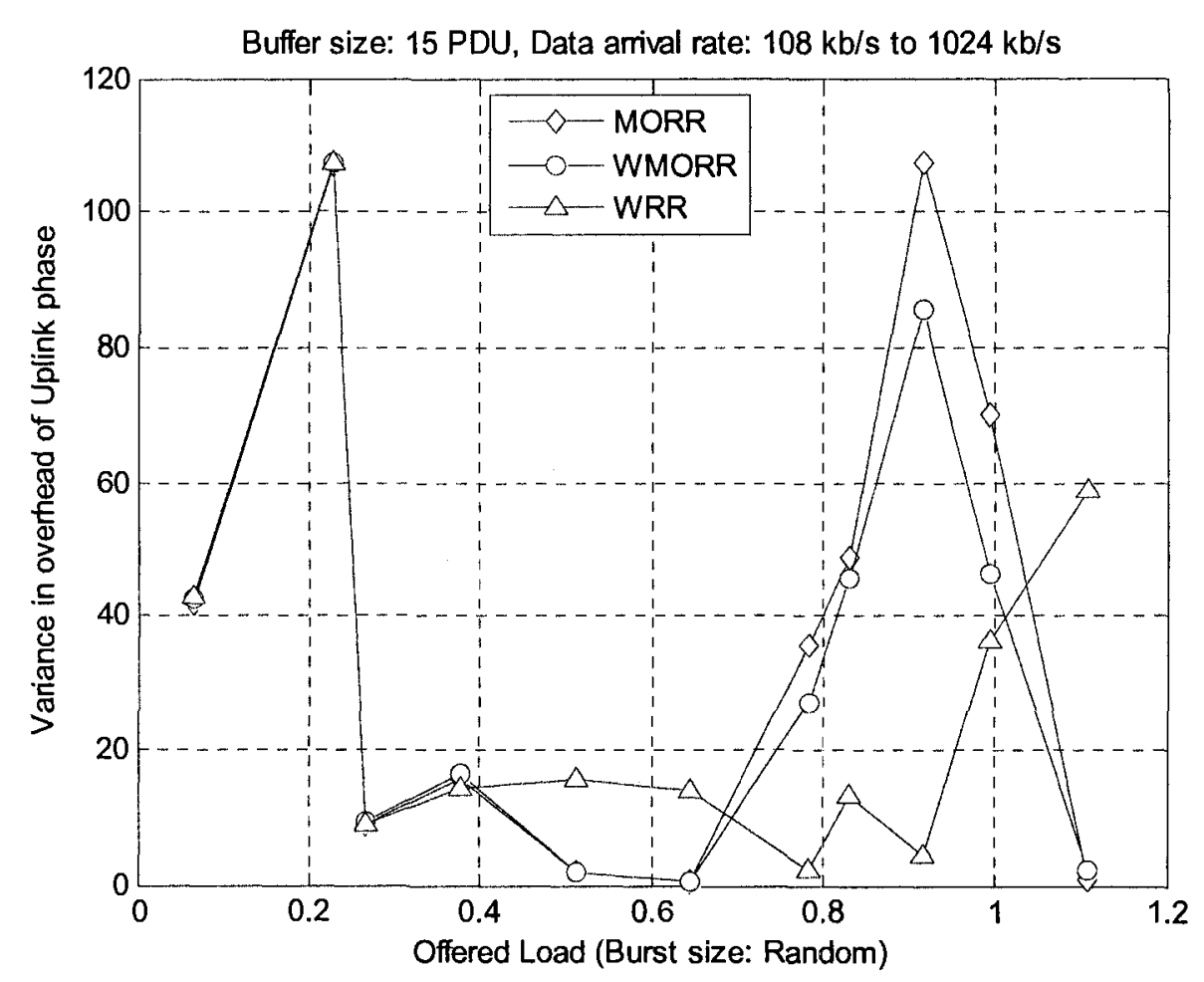

Figure 5.84. Variance in overhead of uplink phase vs offered load for buffer size 15 at random burst.

\section{- Average Overhead in Downlink Phase and its variance}

Fig. 5.85, 5.86 and 5.87 depict the average overhead in downlink phase (Percentage of downlink time) as a function of offered load for the three resource allocation policies, where buffer size is 5,10 and 15 PDUs respectively. The figures show that the MORR and WMORR policies have low overhead compared to the WRR allocation scheme. For low value of offered load the downlink overhead is large as all the arrived PDU gets the transmission oppourtunity and overhead of all the user add up to give a large overhead. But as the load increases arrival rate beomes higher than the service rate and very few of the user get the transmission opportunity. This results very low overhead for MORR and WMORR scheme as according to these schemes when ever an user is visited, system capacity is assigned to it in these schemes. But for the WRR scheme as all users is visited during a TDMA frame, ovherhead of all the user adds up and the summaion continue to increase with the increase of load. 


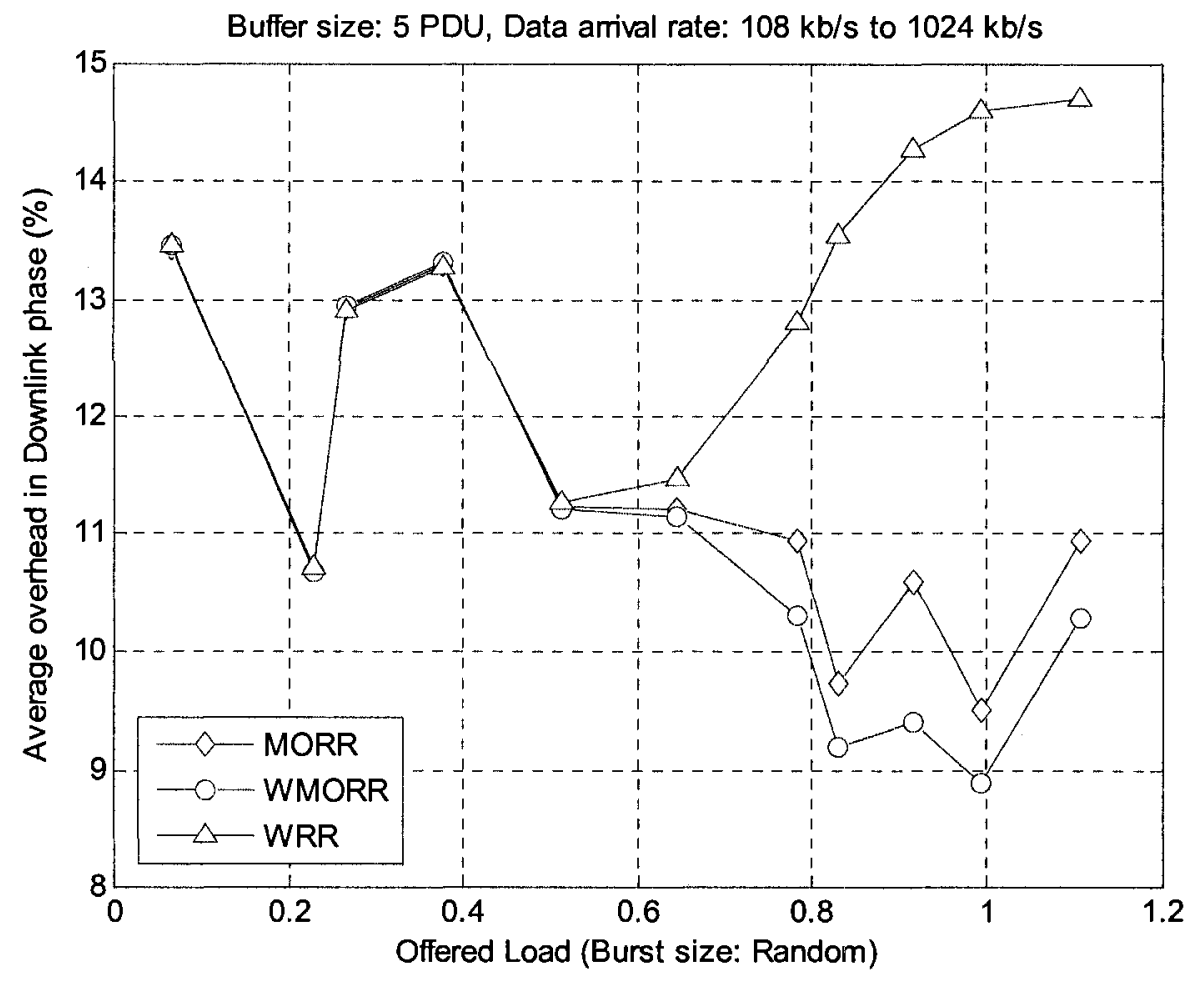

Figure 5.85. Average overhead in downlink phase vs offered load for buffer size 5 at random burst.

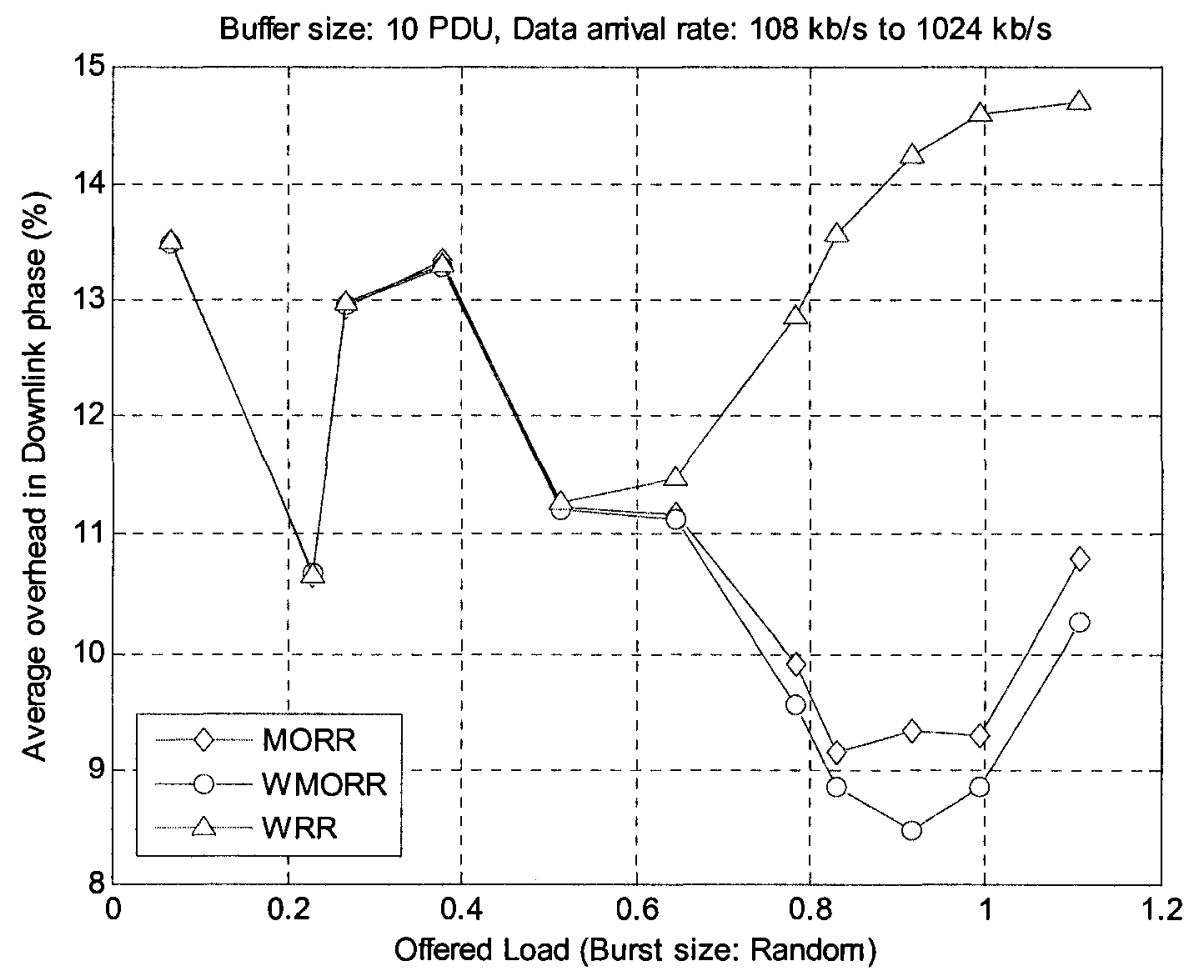

Figure 5.86. Average overhead in downlink phase vs offered load for buffer size 10 at random burst. 


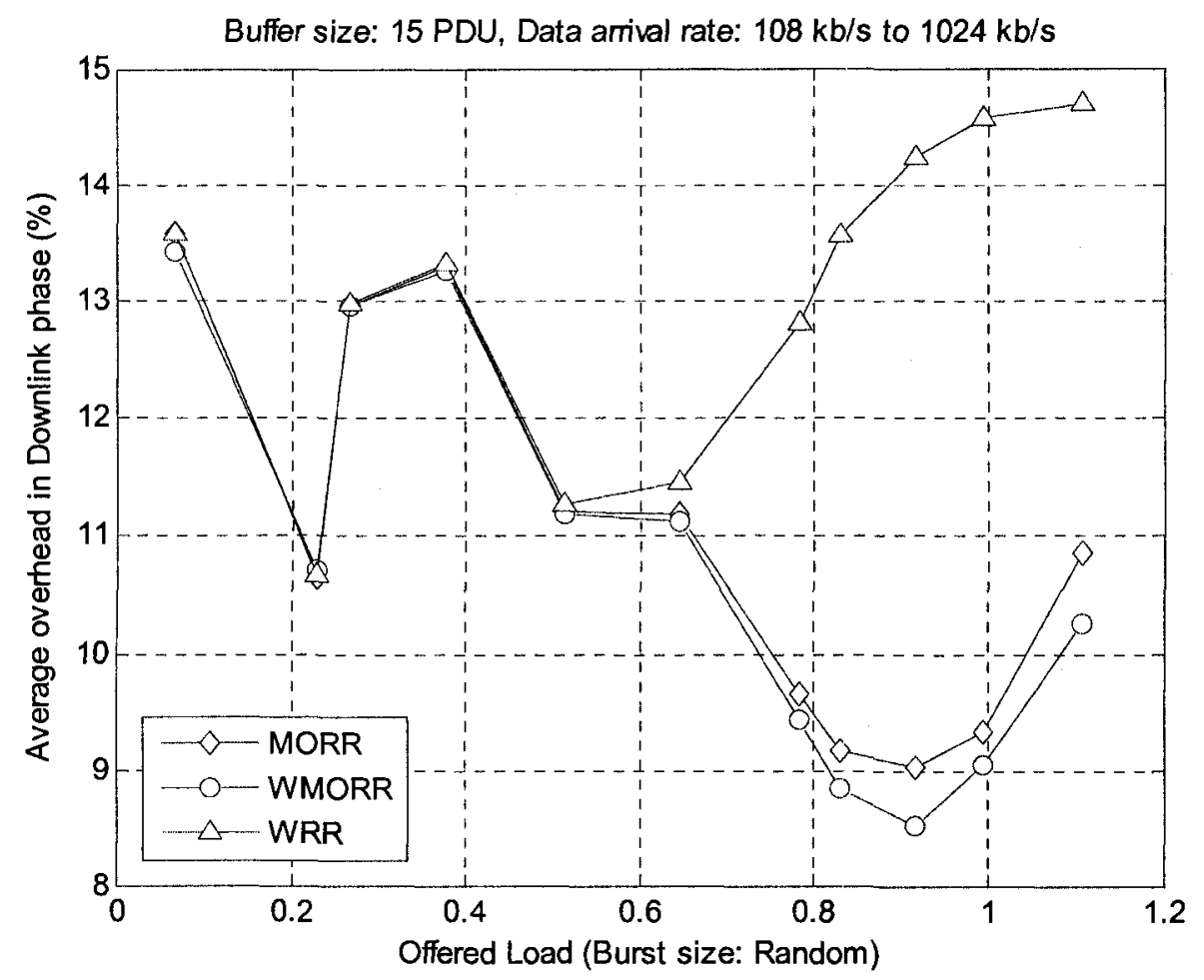

Figure 5.87. Average overhead in downlink phase vs offered load for buffer size 15 at random burst.

Fig. 5.88, 5.89 and 5.90 shows the average overhead (Percentage of total downlink time) in downlink phase as a function of offered load for buffer sizes 5, 10 and 15 PDUs and the figures are for the MORR, WMORR and WRR resource allocation schemes respectively. We notice that for all the schemes change in overhead does not depend on buffer size of the MT. It is due to the fact that here resource is allocated using the number of PDUs for the MTs in the buffer of AP not using the buffer occupancy of MTs. 


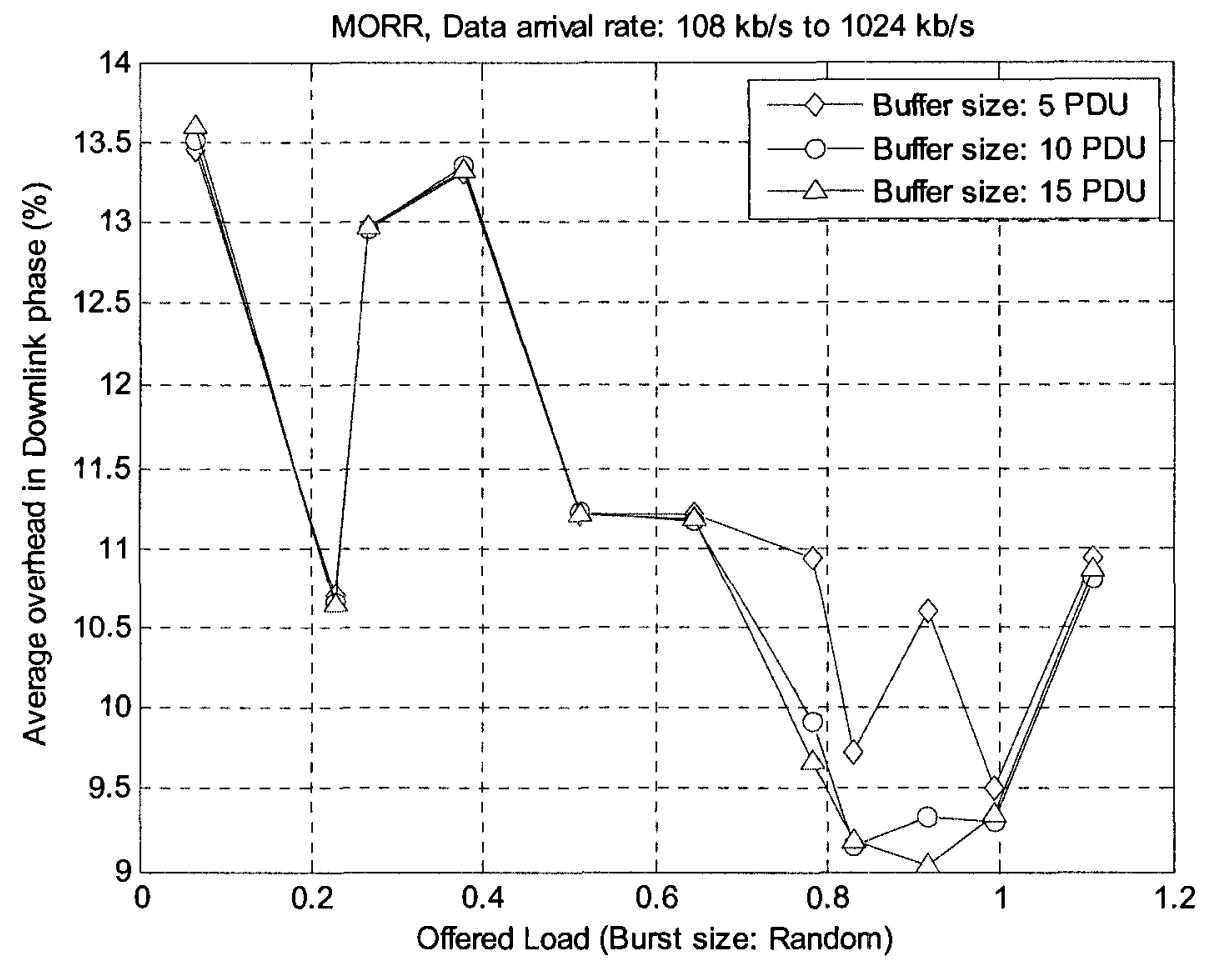

Figure 5.88. Average overhead in downlink phase vs offered load for MORR scheme at random burst.

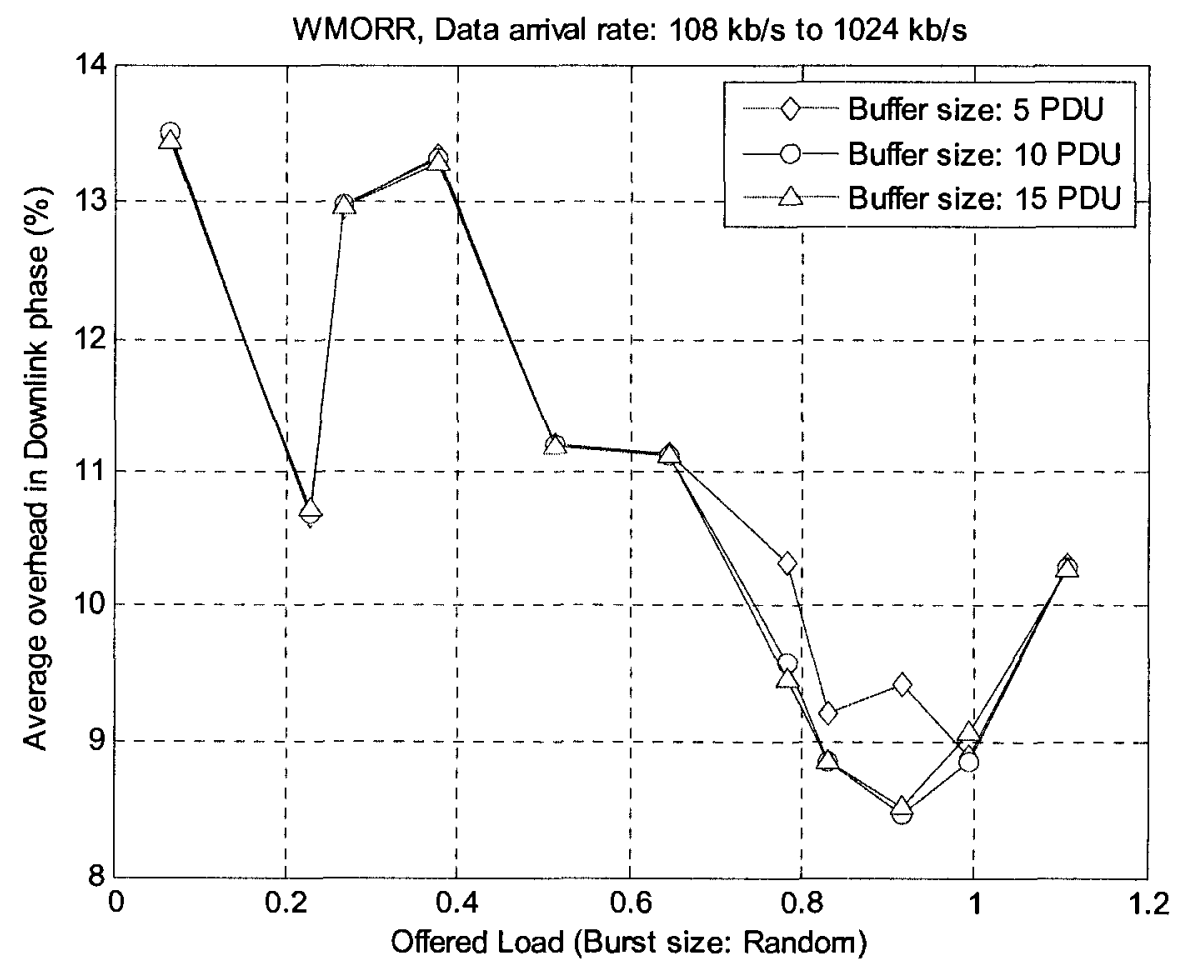

Figure 5.89. Average overhead in downlink phase vs offered load for WMORR scheme at random burst. 


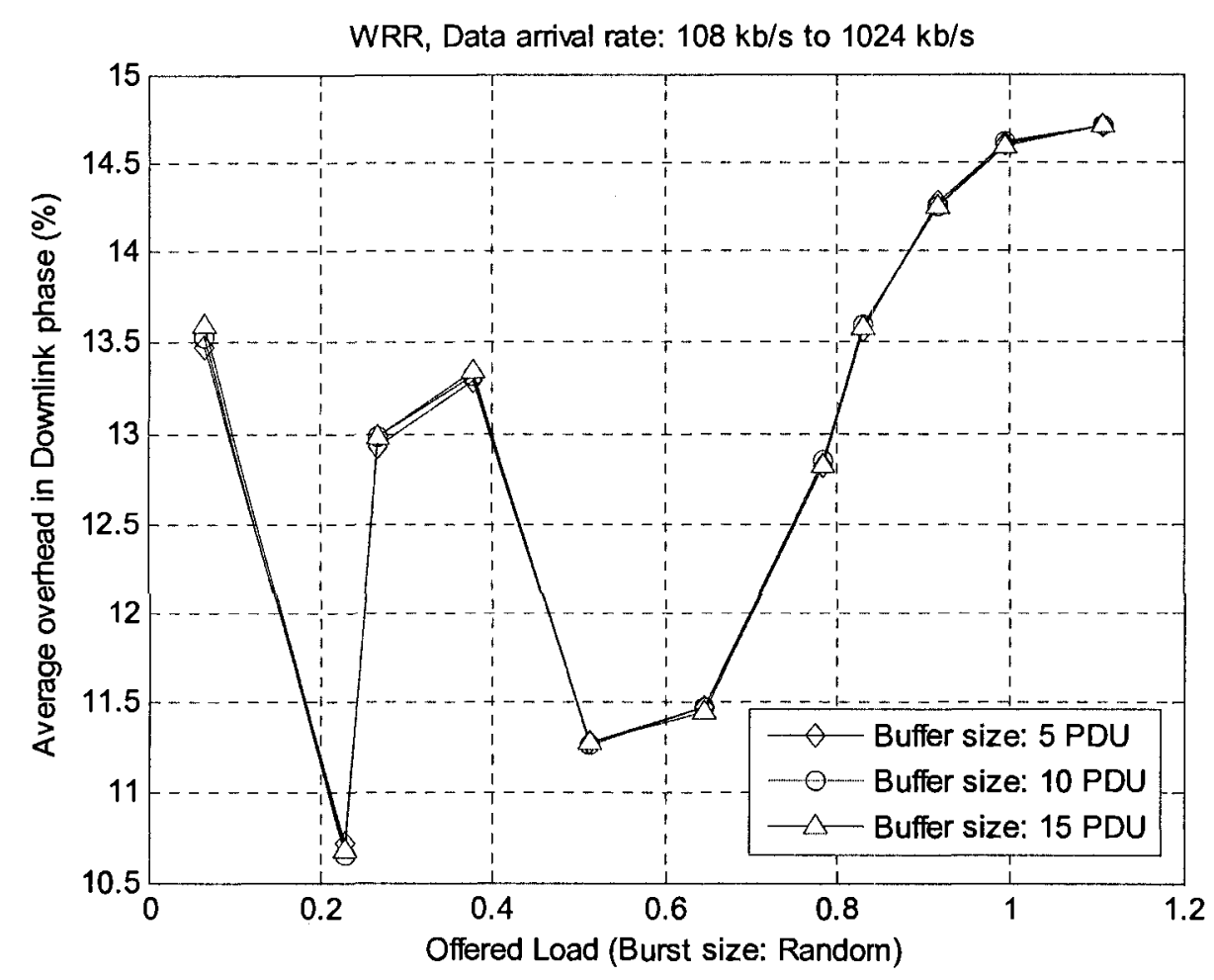

Figure 5.90. Average overhead in downlink phase vs offered load for WRR scheme at random burst.

Variance in overhead of downlink phase is shown in fig. 5.91, 5.92 and 5.93 as a function of offered load for the three resource allocation schemes and the figures represent the results when the buffer size is 5,10 and 15 PDUs respectively. In the figures we noticed that change in variance corresponds to the change in curves of fig. $5.85-5.90$. 


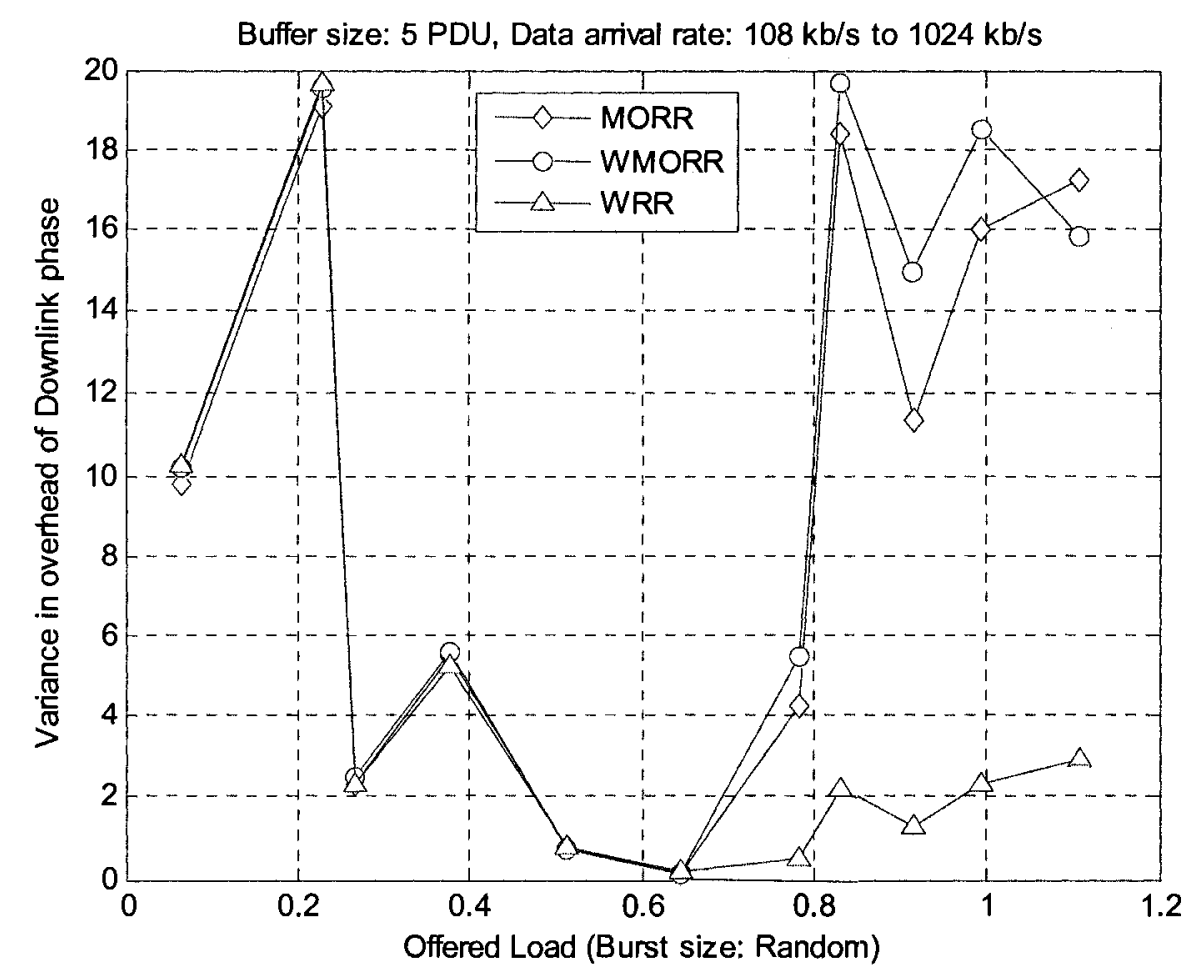

Figure 5.91. Variance in overhead of downlink phase vs offered load for buffer size 5 at random burst.

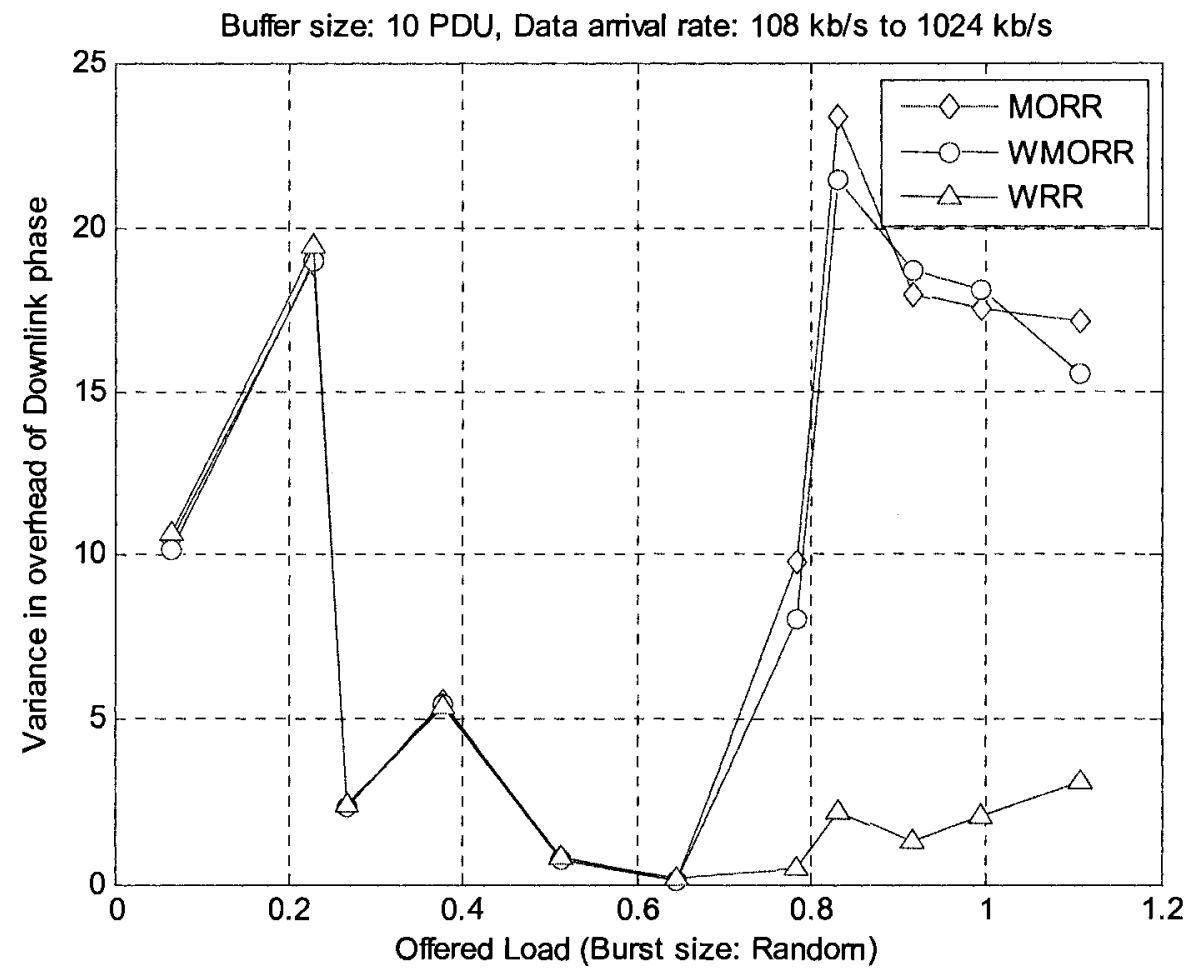

Figure 5.92. Variance in overhead of downlink phase vs offered load for buffer size 10 at random burst. 


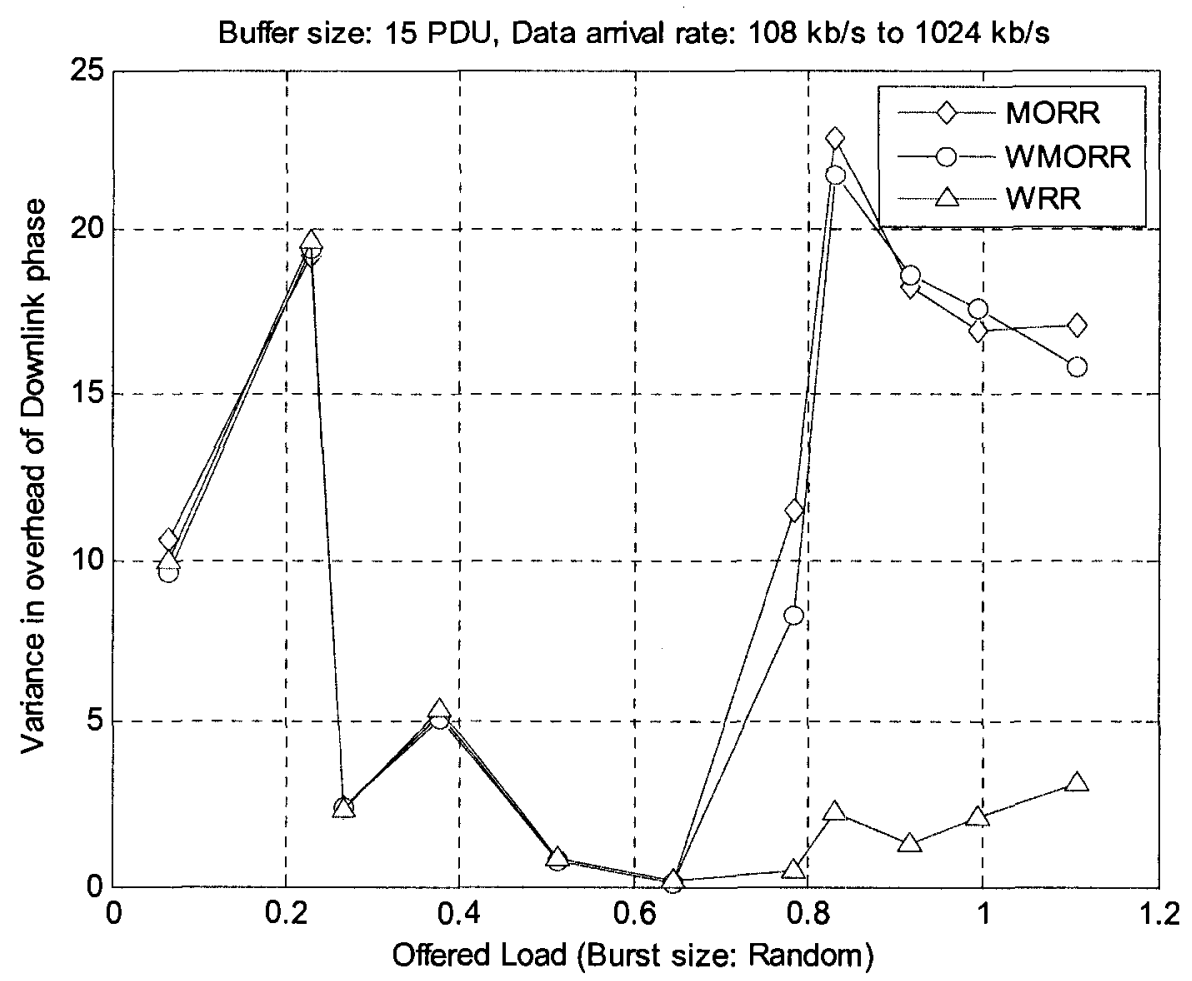

Figure 5.93. Variance in overhead of downlink phase vs offered load for buffer size 5 at random burst. 


\section{Chapter 6: Conclusion}

In this thesis we have simulated a large private WLAN to provide service in a metropolitan area. Our system is cellular in type and we have distributed the users randomly inside each cell. We have considered mobile users with dynamic transmission rates. We have proposed three resource allocation policies to distribute system resource among the users. To measure the performance we have generated a generic simulation program and run a large number of iterations using some realistic assumptions to make our system simple.

We have measured the performance of the system where we used buffer occupancy, probability of buffer overflow, average packet delivery time, overhead in uplink and in downlink phase as performance criteria. From the results we have found that the WRR policy makes lower PDU delivery time for some instance but it has highest overflow, overhead and buffer occupancy. But the WMORR scheme shows lowest buffer occupancy, overhead, overflow and moderate PDU delivery time. Another important thing is that in the WMORR scheme the system is operable at load higher than the WRR scheme. The WMORR also yields the lowest buffer occupancy, an important feature in case of receiving large bursts. So we can conclude that WMORR has the best performance among the three resource allocation policies. These results are also applicable to other high performance TDMA/TDD system such as WiMAX. 


\subsection{Major Contributions}

The major contributions of this thesis are as follows:

- Design of infrastructure multi-clustered private WLAN for urban area.

- Determine physical layer data rate of users depending on distance from Access Point.

- Setting up new rules for resource allocation.

- Evaluating the useful operating region of the network.

- Performance study of HiperLAN/2 and resource allocation schemes.

\subsection{Suggested Future Work}

We suggest the following for future research work:

- For simplificaion we made assumption that mobile terminal cannot move from its associated access-point. In practice it happens and it can be studied in future.

- Serving a mobile node outside the service area of the Access Point using a gratuitous node can be an interesting point to discover in future and resource allocaion for this node can be challanging.

- In this thsis we considered best effort packet delivery for simplificity but in the hiperLAN/2 standard packet delivery with ARQ is also supported, which can be an interesting tropic for future research.

- We considered the case where no packet loss in between AP and CC. But as the link is wireless, packet loss may occur and this can be also included for future study. 
- The effect of other networks in the same service area can also be considered. 
[1] WiMAX : Standards and Security / edited by Syed Ahson, Mohammad Ilyas, CRC Press, c2008

[2] IEEE 802.11-1999, Part 11: Wireless LAN Medium Access Control (MAC) and Physical Layer (PHY) Specificaions, 1999.

[3] IEEE 802.11a-1999, Part 11: Wireless LAN Medium Access Control (MAC) and Physical Layer (PHY) Specificaions, High speed Physical Layer in the $5 \mathrm{GHz}$ Band, 1999.

[4] IEEE 802.11b-1999, Part 11: Wireless LAN Medium Access Control (MAC) and Physical Layer (PHY) Specificaions, High speed Physical Layer Extension in the 2.4 GHz Band, 1999.

[5] IEEE 802.11g-2003, Part 11: Wireless LAN Medium Access Control (MAC) and Physical Layer (PHY) Specificaions, High speed Physical Layer in the 2.4 GHz Band, 2003.

[6] WiMAX : applications / edited by Syed Ahson, Mohammad Ilyas, CRC Press, c2008

[7] ETSI TR 101683 v1.1.1 (2000-02).: Broadband Radio Access Networks (BRAN); HIPERLAN Type 2; System Overview.

[8] ETSI TS 101 761-1: Broadband Radio Access Networks (BRAN); HIPERLAN Type 2; Data link Control (DLC) Layer, Part 1: Basic data transport functions.

[9] ETSI TS 101 761-2: Broadband Radio Access Networks (BRAN); HIPERLAN Type 2; Data link Control (DLC) Layer, Part 2: Radio link Control Protocol Basic functions.

[10] IEEE Standard for Local and Metropolitan Area Networks - Part 16: Air Interface for Fixed Broadband Wireless Access Systems, IEEE std. 802.16-2001, December 2001.

[11] A. Stelter, Random Access and Channel Allocation Scheme for HIPERLAN/2 System, IEEE 16th International Symposium on Personal, Indoor and Mobile Radio Communications 2005.

[12] E. Mingozzi, QoS support by the HiperLAN/2 MAC protocol: a performance evaluation, Cluster Computing vol. 5 (2002) 145-155.

[13] F. M. Delicadio, P. Cuenca, L. Orozco-Barbosa, A QoS-aware WLAN Resource Request Mechanism for Delay Sensitive Traffic, IEEE CNF, Volume 3, 5-8 Sept. 2004 Page(s):2113 2118.

[14] H. H. Choi, G. H. Hwang, D.H. Cho, Adaptive random access and resource allocation scheme based on traffic load in HiperLAN type2 system, IEEE Communications Letters, Volume 7, Issue 4, April 2003 Page(s):192 - 194.

[15] L. Lenzini, E. Mingozzi, Performance evaluation of capacity request and allocation mechanisms for HiperLAN2 wireless LANs, Computer Networks vol. 37 (2001) 5-15.

[16] A Kadelka, A. Masella, Serving IP quality of service with Hiper-LAN/2, Computer Networks vol. 37 (no. 1) (2001) 17-24.

[17] C. Politis, R. Tafazolli, MAC protocol for supporting QoS in All-IP HiperLAN2, in: Proceedings of the Fourth European Wireless Conference (EWO2), Florence, Italy, Feb 2002. 
[18] C. Mangin, G. Froc, R. Rollet, Resource Allocation and Wireless Scheduling Scheme for a HiperLAN/2 System, Mobile Networks and Applications 10, 639-650, 2005.

[19] F. M. Delicadio, P. Cuenca, L. Orozco-Barbosa, QoS-mechanisms for multimedia communications over TDMA/TDD WLANs, Computer Communications 29(2006), 2721 2735.

[20] E'TSI TS 101 475: v1.1.1 (2000-04) Broadband Radio Access Networks (BRAN); HIPERLAN Type 2; Physical (PHY) Layer.

[21] Alberto Leon-Garcia, Probability and Random Processes for Electrical Engineering, 2nd. Ed., Addison Wesley, 1994

[22] X. Zhao, J. Kivinen, P. Vainikainen, Propagation Characteristics for Wideband Outdoor Mobile Communications at $5.3 \mathrm{GHz}$, IEEE Journal on selected areas in communications, Vol. 20, No. 3, April 2002.

[23] S. Armour, A. Doufexi, A. Nix, D. Bull, A study of the Impact of Frequency Selectivity on Link Adaptive Wireless LAN Systems, VTC 2002, Volume 2, 24-28 Sept. 2002 Page(s):738 742 vol. 